University of Louisville

ThinkIR: The University of Louisville's Institutional Repository

Electronic Theses and Dissertations

$7-2013$

\title{
Surface bound PEG-RGDS on PEG-DA hydrogels and its effects on RPE cell morphology and differentiation.
}

Jeremy E. Phillips

University of Louisville

Follow this and additional works at: https://ir.library.louisville.edu/etd

\section{Recommended Citation}

Phillips, Jeremy E., "Surface bound PEG-RGDS on PEG-DA hydrogels and its effects on RPE cell morphology and differentiation." (2013). Electronic Theses and Dissertations. Paper 1132.

https://doi.org/10.18297/etd/1132

This Master's Thesis is brought to you for free and open access by ThinkIR: The University of Louisville's Institutional Repository. It has been accepted for inclusion in Electronic Theses and Dissertations by an authorized administrator of ThinkIR: The University of Louisville's Institutional Repository. This title appears here courtesy of the author, who has retained all other copyrights. For more information, please contact thinkir@louisville.edu. 


\title{
SURFACE BOUND PEG-RGDS ON PEG-DA HYDROGELS AND ITS EFFECTS ON RPE CELL MORPHOLOGY AND DIFFERENTIATION
}

\author{
By \\ Jeremy E. Phillips \\ BS in Bioengineering, University of Louisville, 2013
}

\author{
A Thesis \\ Submitted to the Faculty of the \\ University of Louisville \\ J. B. Speed School of Engineering \\ As Partial Fulfillment of the Requirements \\ For the Professional Degree
}

MASTER OF ENGINEERING

Department of Bioengineering

July 2013 

SURFACE BOUND PEG-RGDS ON PIBG-DA HYDROGELS $\triangle$ ND ITS EFFECTS ON RPE CELL MORPHOLOGY AND DIFHERENTIATION

Submitted by:

Jeremy F. Phillips

A Thesis Approved On

Ditle

by the Following Reading and Examinazion Committee:

Dr. Robert S Keynton, Bioengineering Thesis Director

Dr. Andrea S. Gobin, Bioengineering

Dr. Martin G. O'Toole. Bioenginceting

Dr. Stuart I Williams. Mechanical Engineering 


\title{
ACKNOWLEDGEMENTS
}

First of all, I would like to thank the following people for their support and contributions to this research.

\author{
Dr. Andrea Gobin \\ Dr. Robert Keynton \\ Dr. Martin O’Toole \\ Dr. Palaniappan Sethu \\ Dr. Patricia Soucy \\ Dr. Stuart Williams \\ Betty Nunn
}

I would also like to thank my wonderful wife Sara Phillips for her support, encouragement and inspiration. Finally, I would like to thank God for the grace and perseverance to finish. Without Him, I would not be who I am today, both in person and profession. 


\begin{abstract}
Age related macular Degeneration is a retinal condition resulting in visual impairment and central vision loss caused by dysfunction of the Bruch's membrane and the retinal-pigmented epithelium. . A novel treatment idea proposes that these layers may be repaired through replacement using a synthetic Bruch's membrane, such as modified poly(ethylene glycol) commonly known as PEG, allowing regrowth of the RPE layer in vivo or through inclusion of a functioning RPE layer when implanting the membrane. Modifying poly(ethylene glycol) with surface bound adhesion peptides such as RGDS provides an excellent biomaterial scaffold in which RPE cells may repopulate and regain their appropriate functions. The purpose of the proposed research is to evaluate the efficiency of the surface bound adhesion peptide, RGDS, and the effect of RGDS concentration on cellular confluency and morphology in both ARPE-19 and PRPE cell lines on modified PEG hydrogels. Using fluorescent and confocal microscopy, it was found that between 4 and 5 percent of the total coating solution was effectively bound to the hydrogel surface. Cellular morphology using immunocytochemistry staining of zonula occluden, or ZO-1, also demonstrated morphological characteristics are obtainable using visual parameters such as cell circularity. Even though differentiation was shown to be lacking when compared to fibronectin controls, the results indicate that all concentrations tested proved effective in providing optimal cellular confluency.
\end{abstract}


TABLE OF CONTENTS

\begin{tabular}{|c|c|c|}
\hline \multirow{2}{*}{\multicolumn{3}{|c|}{ APPROVAL PAGE }} \\
\hline & & \\
\hline \multicolumn{2}{|c|}{ ACKNOWLEDGEMENTS } & iii \\
\hline \multicolumn{2}{|c|}{ ABSTRACT } & iv \\
\hline \multicolumn{2}{|c|}{ NOMENCLATURE } & vii \\
\hline \multicolumn{2}{|c|}{ LIST OF TABLES } & viii \\
\hline \multicolumn{2}{|c|}{ LIST OF FIGURES } & ix \\
\hline \multirow[t]{5}{*}{ I. } & INTRODUCTION & 1 \\
\hline & A. OBJECTIVE & 2 \\
\hline & B. HYPOTHESIS & 2 \\
\hline & C. SPECIFIC AIMS & 3 \\
\hline & D. SIGNIFICANCE OF STUDY & 4 \\
\hline \multirow[t]{14}{*}{ II. } & BACKGROUND & 6 \\
\hline & A. ANATOMY OF THE EYE & 6 \\
\hline & 1. GENERAL EYE ANATOMY & 6 \\
\hline & 2. RETINAL PIGMENTED EPITHELIUM & 10 \\
\hline & 3. BRUCH'S MEMBRANE & 14 \\
\hline & B. MACULAR DEGENERATION & 15 \\
\hline & 1. PATHOLOGY & 16 \\
\hline & 2. CURRENT TREATMENTS & 18 \\
\hline & 3. PROPOSED SOLUTION & 22 \\
\hline & C. HYDROGELS & 23 \\
\hline & 1. HYDROGEL HISTORY & 23 \\
\hline & 2. POLY(ETHYLENE GLYCOL) HYDROGELS & 24 \\
\hline & D. ADHESIVE LIGANDS & 26 \\
\hline & 1. RGDS & 27 \\
\hline \multirow[t]{8}{*}{ III. } & METHODS AND MATERIALS & 29 \\
\hline & A. CELL CULTURE & 29 \\
\hline & 1. ARPE-19 & 30 \\
\hline & 2. PRPE & 31 \\
\hline & B. PEG-DA SYNTHESIS & 31 \\
\hline & C. PEG-RGDS SYNTHESIS & 33 \\
\hline & D. PEG-RGDS-FLUOR 488 SYNTHESIS & 34 \\
\hline & E. PEG-DA HYDROGEL FORMATION & 34 \\
\hline
\end{tabular}


F. HYDROGEL COATING PROCEDURE 35

G. QUANTIFICATION OF PEPTIDE COATING USING RINSE 36 SUPERNATANT

H. QUANTIFICATION OF PEPTIDE COATING USING CONFOACL MICROSCOPY

I. PREPARATION OF RPES ON HYDROGELS 38

J. PREPARATION OF RPES ON FIBRONECTIN 39

K. PHASE CONTRAST MICROSCOPY 39

L. IMMUNOSTAINING PROCEDURE 40

M. CONFOCAL MICROSCOPY 41

N. ZO-1 ANALYSIS $\quad 42$

O. ACTIN ANALYSIS 43

IV. RESULTS AND DISCUSSION 45

A. QUANTIFICATION OF PEG-RGDS COATING (FLUORESCENCE) 45

B. QUANTIFICATION OF PEG-RGDS COATING (CONFOCAL) 48

C. QUANTIFICATION OF CELLULAR CONFLUENCY ON 51 HYDROGELS

D. DETERMINATION OF RPE DIFFERENTIATION: ZO-1 CELL 56 CIRCULARITY

E. DETERMINATION OF RPE DIFFERENTIATION: ZO-1 CELL 61 PERIMETER

F. DETERMINATION OF RPE DIFFERENTIATION: ZO-1 CELL 64 AREA

G. DETERMINATION OF RPE DIFFERENTIATION: ZO-1 ASPECT 66 RATIO

H. DETERMINATION OF RPE DIFFERENTIATION: ACTIN 70

FILAMENT LENGTH AND ORIENTATION

V. CONCLUSIONS

VI. RECOMMENDATIONS 75

REFERENCES CITED

APPENDIX I: GEL CONCENTRATION QUANTIFICATION IMAGES 82

APPENDIX II: GEL CONFLUENCY IMAGES 91

APPENDIX III: CONFOCAL MICROSCOPY IMAGES 182

CURRICULUM VITAE 235 


\section{NOMENCLATURE}

AMD -Age-related macular degeneration

ARPE-19 -Human adult retinal pigmented epithelial cells

BSA- Bovine serum albumin

BlamD- diffuse drusen internal to RPE basement membrane basal laminar deposit

BlinD- diffuse drusen external to RPE basement membrane basal linear deposit.

CNV-Choroidal neovascularization

DAPI- 4',6-diamidino-2-phenylindole

DCM- Dichloromethane

DMEM/F-12- Dulbecco's modified eagle medium: nutrient mixture F-12

ECM- Extracellular matrix

FBS- Fetal bovine serum

FGF- Fibroblast growth factor

NVP- N-vinylpyrrolidone

PEDF- Pigment epithelium-derived factor

PEG- Poly(ethylene glycol)

PEG-DA- Poly(ethylene glycol) diacrylate

PEG-RGDS- Poly(ethylene glycol) covalently bound to peptide Arg-Gly-Asp-Ser

PEG-RGDS-Fluor 488- PEG-RGDS fluorescently labeled with AlexaFluor 488

PLA- poly(lactic acid)

PLGA- poly(DL-lactic-co-glycolic acid)

PRPE- (Primary) porcine retinal pigmented epithelial cell

RGDS- Arginine-glycine-aspartic acid-serine (Arg-Gly-Asp-Ser) peptide

$\mathbf{R}-\mathbf{P}$-rhodamine-phalloidin

RPE - Retinal pigmented epithelial cell

TEA- Triethyl amine

ZO-1 -Zonula Occludens 


\section{LIST OF TABLES}

$\begin{array}{lll}\text { TABLE I } & \text { Standard Curve Analysis } & 46\end{array}$

TABLE II Quantification of PEG-RGDS Coating Fluorescence 47

TABLE III Average $\mu \mathrm{g} / \mathrm{cm}^{2}$ p-values $\quad 47$

TABLE IV Average Greyscale Intensity p-values 50

TABLE V ARPE-19 Confluency p-values $\quad 53$

TABLE VI PRPE Confluency p-values $\quad 55$

TABLE VII Gel and Cell Number Count $\quad 57$

TABLE VIII Circularity p-values $\quad 59$

$\begin{array}{lll}\text { TABLE IX } & \text { ANOVA for Circularity } & 60\end{array}$

TABLE X $\quad$ Tukey's Test for Circularity vs. Concentration 60

TABLE XI Perimeter p-values $\quad 61$

$\begin{array}{lll}\text { TABLE XII } & \text { ANOVA for Perimeter } & 63\end{array}$

TABLE XIII Tukey's Test for Perimeter vs. Concentration 63

$\begin{array}{lll}\text { TABLE XIV Area p-values } & 64\end{array}$

TABLE XV ANOVA for Area $\quad 65$

TABLE XVI Tukey's Test for Area vs. Concentration 65

TABLE XVII Aspect Ratio p-values $\quad 67$

TABLE XVIII ANOVA for Aspect Ratio 68

TABLE XIX Tukey's Test for Aspect Ratio vs. Concentration 68 


\section{LIST OF FIGURES}

\begin{tabular}{|c|c|c|}
\hline FIGURE 1 & Eye Anatomy & 7 \\
\hline FIGURE 2 & Bruch's Membrane in relation to RPEs and choroid & 15 \\
\hline FIGURE 3 & Amsler grids & 16 \\
\hline FIGURE 4 & Poly(ethylene glycol) & 25 \\
\hline FIGURE 5 & Standard Curve of PEG-RGDS-Fluor 488 Fluorescence & 46 \\
\hline FIGURE 6 & PEG-RGDS-Fluor 488 Hydrogels & 49 \\
\hline FIGURE 7 & Concentration vs. Average Greyscale Intensity & 50 \\
\hline FIGURE 8 & ARPE-19 10 $\mu \mathrm{mol} / \mathrm{mL}$ sample & 52 \\
\hline FIGURE 9 & ARPE-19 Attachment to Surface & 52 \\
\hline FIGURE 10 & PRPE $10 \mu \mathrm{mol} / \mathrm{mL}$ sample & 54 \\
\hline FIGURE 11 & PRPE Attachment to Surface & 54 \\
\hline FIGURE 12 & PRPE 10-2-1 & 56 \\
\hline FIGURE 13 & PRPE FN-2-2 and ARPE FN-1-4 & 58 \\
\hline FIGURE 14 & Circularity by Group & 59 \\
\hline FIGURE 15 & Perimeter by Group & 61 \\
\hline FIGURE 16 & Area by Group & 64 \\
\hline FIGURE 17 & Aspect Ratio by Group & 67 \\
\hline FIGURE 18 & Actin Expression on Fibronectin controls & 72 \\
\hline FIGURE 19 & Actin Expression in Differentiated RPEs & 72 \\
\hline \multicolumn{2}{|c|}{ FIGURE A1.1-A1.16 } & Appendix I \\
\hline \multicolumn{2}{|c|}{ FIGURE A2.1-A2.90 } & Appendix II \\
\hline \multicolumn{2}{|c|}{ FIGURE A3.1-A3.52 } & Appendix III \\
\hline
\end{tabular}




\section{INTRODUCTION}

Age-related macular degeneration (AMD) is a degenerative eye disorder that commonly effects people over the age of 50 (National Eye Institute, 2009). It is the leading cause of blindness in industrialized nations (Sun et al., 2001) This disease affects over 1.75 million people in the United States and is expected to increase by $50 \%$ by the year 2020 (The Eye Diseases Prevalence Research Group, 2004). Prevalence of the disease also dramatically increases with age. An estimated 8 million Americans over the age of 65 are at high risk to develop AMD, of which 1.3 million will develop the condition within the next 5 years (National Eye Institute, 2011).

Multiple treatments are currently being developed for AMD within the field of bioengineering (Lee et al., 2005)(Kubota et al., 2005)(Binder et al., 2004). One proposed treatment removes the damaged Bruch's membrane and replaces it with a synthetic scaffold, which functions similarly to the membrane extracellular matrix (Kubota et al., 2005). This allows introduction of a newly seeded Bruch's membrane (Kubota et al., 2005) or migration of cells from the healthy portions of the retina to repair the diseased area (Binder et al., 2004). Of these scaffold materials, poly(ethylene glycol) (PEG) is currently being pursued as a potential candidate due to its desirable mechanical properties, ability to polymerize in situ, and inability to promote cellular adhesion (Gombotz, 2004). PEG also has the ability to control its density and elastic modulus by changing the concentration and molecular weight of the polymer, which allows for the material to be specifically tuned for use in repairing this tissue (Brandl et 
al., 2007). Mann et al. (2001) demonstrated the ability to control adhesion on PEG hydrogels using covalently modified peptide sequences, such as RGDS, for migration and proliferation of smooth muscle cells; thus, enabling controlled tissue ingrowth. However, to date, the majority of Bruch's membrane substitute materials developed do not provide the necessary signaling for controlled morphology; thus, there is a need for an alternative solution.

\section{A. Objective}

The objective of this research is to create and characterize a substitute Bruch's membrane material using PEG hydrogels with varying concentrations of surface bound RGDS to promote adhesion of the retinal pigmented epithelial (RPE) cells while displaying morphology linked to a differentiated state.

\section{B. Hypothesis}

The hypothesis of this study is that increasing concentrations of RGDS on PEG hydrogels will significantly promote morphological expression, including overall cell coverage, cell circularity, cell area, actin filament length and actin filament orientation, related to RPE-specific differentiation in both adult retinal pigmented epithelial (ARPE-19) and primary RPE (PRPE) cell lines. This hypothesis has been evaluated by the completion of the objectives and specific aims listed below. 


\section{C. $\underline{\text { Specific Aims }}$}

\section{Specific Aim 1-1: Quantification of PEG-RGDS Coating (Fluorescence)}

PEG hydrogels were polymerized and coated with mixtures of RGDS and RGDS-Fluor 488 at a 10:1 ratio. Coating concentrations of 20,10, 5 and 0 $\mu \mathrm{mol} / \mathrm{mL}$ were created and polymerized. Rinses from each individual gel were then collected and analyzed using a fluorometer. This was used to determine an average concentration of PEG-RGDS coating per $\mathrm{cm}^{2}$ for each coating level.

\section{Specific Aim 1-2: Quantification of PEG-RGDS Coating (Confocal)}

Also under specific aim one, RGDS-Fluor 488 coated gels were analyzed using confocal microscopy to further observe gel coating characteristics, homogeneity and deviation of the coating intensity between gel concentrations.

\section{Specific Aim 2: Quantification of Cellular Confluency on Hydrogels}

Surface coverage for PEG-RGDS hydrogels was also observed. This was completed using phase contrast imaging for each coating concentration, fibronectin control and RPE cell type. Images were analyzed using ImageJ (Version 1.47; NIH 2004) and compared for significant differences.

\section{Specific Aim 3: Determination of RPE Differentiation using ZO-1 Staining}

In order to observe morphological properties of the RPEs on PEG-RGDS coated hydrogels, each gel coating concentration, RPE line and controls were 
stained for ZO-1 using immunocytochemistry techniques. Images were obtained using confocal microscopy and analyzed for circularity, area, cell perimeter and aspect ratio in each selected cell using ImageJ. These parameters were then used to compare gel groups and RPE lines to their respective fibronectin controls.

\section{Specific Aim 4: Determination of RPE Differentiation using Actin Staining}

Samples were also stained with rhodamine-phalloidin to obtain imaging of actin filaments within the RPEs. Cells within each image were then selected and analyzed using ImageJ to determine average actin filament length compared to each sample group. Orientation of fibronectin controls were also compared with the coated gels to further compare morphological properties of each group.

\section{Significance of Study}

This study is significant for a number of reasons. First, this is the one of only a few studies focused on using poly(ethylene glycol) modified with adhesive peptides for development of a synthetic Bruch's membrane. Only one previous study based on the same material is known, of which is the precursor to this study (Scherzer 2010). Although this study is based on a previous idea, the method of material synthesis is novel. This study focuses on the development of surface modified PEG hydrogels compared to homogeneously distributed concentrations of adhesion ligands. This technique allows for further refinement and control of the concentrations of peptide 
exposed, providing a more accurate quantification of the required amount of adhesive ligand per gel area.

This study is also significant in the method used to compare morphology of RPE cells. Using parameters based on cell staining such as circularity, area, perimeter and aspect ratio allows for the introduction of more quantifiable parameters to determine the amount of differentiation of a cell. This provides a numerical standard in which further studies in RPE morphology can be compared to determine the closeness of the confluent cell layer on the modified material to a more natural epithelial phenotype. 


\section{BACKGROUND}

\section{A. Anatomy of The Eye}

The eyes are highly complex organs of the body responsible for capturing light and translating it into electrochemical impulses. As light enters the eye, it is focused using the cornea and lens. This focused light passes through the vitreous humor and is then projected onto the retina. At the retina, light is translated into optic nerve signals by rods and cones. These signals are sent from the photoreceptors to the visual cortex in the brain through the optic nerve and are then further interpreted into the sense of vision.

\section{General Eye Anatomy}

A general anatomical representation of the eye is presented in Figure 1. The general anatomy of the eye consists of three primary layers. The outer layer is composed of the cornea, sclera and optic nerve. The cornea, along with the lens, refracts light and allows for the eye to focus. It is composed primarily of connective tissue and a thin surface epithelial layer (Shier et al., 2012). About two thirds of the overall refractive power of the eye comes from the cornea, due to the high refractive index differential from air (Guyton and Hall, 2011). The sclera is the protective fibrous layer composed of disorganized elastic and collagenous fibers (Shier et al., 2012). The 
sclera also provides protection for the eye as well as an anchor point for extrinsic musculature. The optic nerve is located in the back of the eye and transmits signals from the rods and cones to the visual cortex in the brain.

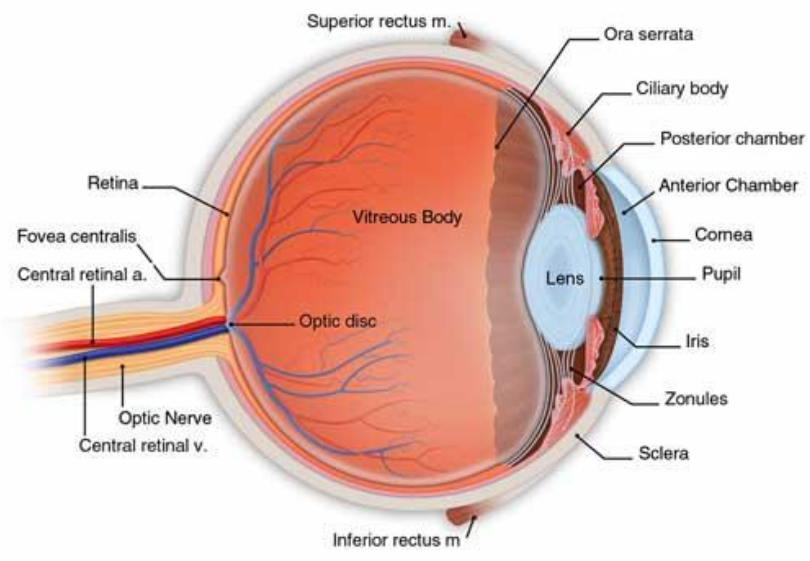

FIGURE 1- Eye Anatomy (Larson Eye Center, 2012)

The choroid coat, ciliary body, lens and the iris form the middle layer of the eye. The choroid is the vascularized section of the eye, allowing for nourishment of the retina (Shier et al., 2012). It also contains melanocytes that absorb excess light within the eye. The ciliary body is the thickest part of the eye and produces aqueous humor. Suspensory ligaments also extend from the ciliary processes to anchor the lens into position. These in combination with the ciliary muscles allow for the eye to focus by altering the shape of the lens using tension. The lens itself lies directly behind the pupil and is composed of epithelial lens fibers. The iris controls the overall amount of light that reaches the retina. It is composed of mostly connective tissue and smooth muscle fibers.

The innermost layer of the eye is the retina. This layer is the light sensitive portion of the eye and allows for chemical signal transmission by converting the light into electrochemical signals which traverse the optic nerve to the visual cortex. The 
retina is mostly transparent to allow for absorption of light by the photoreceptors (Shier et al., 2012). The retina is integrated with the optic nerve and the central area of the retina is called the macula lutea. Within the macula lutea is the fovea centralis, which is the region where the sharpest vision is produced. Medial to the macula lutea is the optic disc, where nerve fibers from the retina join the optic nerve to transmit signals to the visual cortex. Central arteries and veins also pass through the optic disc.

The retina can be divided into nine total layers (Guyton and Hall, 2012). Starting from the inside of the eye is the inner limiting membrane. This layer functions as a barrier between the retina and vitreous humor. The next layer is the ganglionic layer, which contains ganglion cells within the retina that transmit signals from the retina to the optic nerve.

After the ganglionic layer is the inner plexiform layer that contains interplexiform cells which transmit negative feedback signals from the outer plexiform layer to the inner plexiform layer (Guyton and Hall 2012). This feedback mechanism is believed to control the spread of the visual signals by horizontal cells and control the degree of contrast in an image.

Next are the inner nuclear and outer plexiform layers, which contain the nuclei of the majority of cell types used in the transmission of visual signals from the photoreceptors (Guyton and Hall, 2012). These cell types include horizontal, bipolar and amacrine cells. Horizontal cells specifically transmit signals horizontally from the rods and cones to bipolar cells. Bipolar cells transmit signals vertically from the photoreceptors as well as horizontal cells to ganglion or horizontal cells in the inner plexiform layer. Amacrine cells transmit signals in two directions, either from bipolar 
to ganglion or horizontally within the inner plexiform layer to other amacrine cells. About thirty types of amacrine cells have been identified, with only about a half dozen of these being characterized. Each of these amacrine types plays an important role in visual signal stimulation, inhibition and analysis.

The outer nuclear layer contains the bodies of the photoreceptors. Below the outer nuclear layer is the layer of rods and cones projecting to the pigment. Rods and cones are the light sensitive portion of the eye with the rods controlling both light intensity and black and white vision while the cones are responsible for color vision (Guyton and Hall, 2012). When either of these photoreceptors are excited by a light wave transmitted through the eye, an electrical impulse is generated which travels through the connected nerve fibers, described above, and to the optic nerve fibers for final translation into vision.

Both rods and cones can be broken into four functional segments; the outer segment, the inner segment, the nucleus and the synaptic body. The outer segment contains most of the light sensitive photochemicals. For the rods, the photochemical is rhodopsin. For cones, one of three color pigments are used, all functioning in different spectral areas. These pigments are red (erythrolabe), green (chlorolabe) and blue (cyanolabe) (Shier et al., 2012). The inner segment contains the cytoplasm and its respective organelles. The synaptic body connects to each of the different neuron types within the retina.

Lastly, the pigmented layer prevents light reflection from within the eye via increased levels of melanin, allowing for clear images (Guyton and Hall, 2012). This layer also stores a large amount of vitamin A, used in the formation of rhodopsin. 


\section{Retinal Pigmented Epithelium}

Moving further through the retina, the next layer of specialized cells is the retinal pigmented epithelium (RPE). This layer of cells acts as a functional intermediary between the choroid and the neural retina (Grierson et al., 1994). RPEs are typically brown in color due to deposits of melanin, lipofuscin and other pigments (Lu et al., 2001) The high concentration of these pigments allow for absorption of light,

preventing reflection from within the eye (Mecklenburg and Schraermeyer, 2007). The size of RPEs in vivo has been well documented and ranges between 10-60 $\mu \mathrm{m}$. (Roorda et al., 2007)(Lu et al., 2001). This number can vary significantly with age as well as location. Cells near the macula, the central portion of the eye, typically size within the $10-14 \mu \mathrm{m}$ range while the periphery has been measured up to $60 \mu \mathrm{m}$ (Lee et al., 2007).

This epithelial layer is intimately attached to the outer segment tips of the photoreceptors on its apical side while the basal sides rest on a basement membrane formed above the Bruch's membrane (Grierson et al., 1994). RPEs are polarized and involved in multiple activities vital to visual function, such as phagocytosis of used rod and cone outer segments, metabolizing vitamin A, transporting metabolites and forming the blood-retinal barrier (Grierson et al., 1994). Each RPE cell supports approximately 40 photoreceptors by transporting cellular metabolites as well as nutrients from the choricocapillaris (Forrester et al., 2002).

In order to form a functional blood-retinal barrier, zonula occluden, commonly known as tight junctions are formed between RPE cells to prevent leakage through the intercellular spaces (Grierson et al., 1994). Another essential junction includes zonulae 
adherens, or intermediate junctions, which promote adhesive properties and maintenance of the polygonal shape of RPEs as well as help organize the superficial web of actin microfilaments (Sandig and Kalnins, 1988). Gap junctions allow for lowresistance pathways of ions and metabolites between the cells (Hudspeth and Yee, 1973). Actin structure also plays an important role in the morphology of RPE cells. Actin is involved in many cellular processes, including establishing morphology, migration, organelle transport, cellular division and support of cell-cell junctions (Karp et al., 2008). Actin distribution is commonly used for evaluation of RPE morphology (Burke et al., 2008). This is due due to its circumferential orientation around epithelial RPE cells (Lee, Fishman and Bent, 2007). Re-organization of RPE cells from a migratory to epithelial state includes reorganization of actin filaments into peripheral bands (Burke et al., 2008).

These cells are derived from the neuroepithelial cells of the neural plate during development and transdifferentiation into neuroretinal tissue is rapidly lost in mammals, although fibroblast growth factor (FGF) has been shown to stimulate neuroretinal regeneration in chick embryos (Grierson et al., 1994). Interestingly enough, RPE have been shown to transdifferentiate past development in salamanders as iris or lens epithelium. (Grierson et al., 1994). Not only do RPEs contribute to the formation of the Bruch's membrane, but also to the development of the scleral coat (Grierson et al., 1994). Photoreceptor outer segments have also shown not to develop until contact between the neural and epithelial layers are established by the RPE (Hollifield and Witkovsky, 1974). 
RPE cells are extremely limited in replicative ability in situ. It is believed that inhibition is caused by the gap junction system (Grierson et al., 1994). Since these cells are suspended in proliferation by contact inhibition, RPE expansion depends on the enlargement of individual cells. This is especially the case around the periphery, where multi-nucleated RPE cells can measure up to $60 \mu \mathrm{m}$ in diameter compared to the typical $14 \mu \mathrm{m}$ (Grierson et al.,1994). Once contact inhibition is removed due to injury, proliferation of the RPE layer will resume.

Two phenotypic styles of RPEs can form following injury. One form resembles morphology of a large macrophage while the other is elongated, bipolar and fibroblastic (Grierson et al., 1994). Macrophage forms are typical in cases of proliferative vitroretinopathy (PVR) and inhabit the vitreal cavity. These phenotypic expressions in altered circumstances rapidly revert to an epithelioid phenotype, meaning the changes in morphological properties is only metaplasia (Grierson et al., 1994). Once regions of high density and tight packing are obtained, gap junctions form between the adjacent cells. Extracellular matrix (ECM) materials are also synthesized, such as fibronectin (Grierson et al., 1994). Although RPEs may revert back to epithelioid morphologies, establishing an effective mosaic proves to be more difficult both in vitro and in vivo. In culture, RPEs often lose pigmentation and do not usually re-pigment until weeks post confluency (Burke et al., 2008). This extended restructuring process is also seen in $\mathrm{N}$ cadherin formation at cell-cell junctions (McKay et al., 2007). Both N-cadherin and Ecadherin concentrations were shown to be consistent between fibroblastic and epithelial phenotypes, but were reorganized depending on the morphology (McKay et al., 2007). When observing changes in actin structures, settled RPEs produce a diffuse pattern 
around the periphery while migratory forms produce filamentous fibers and finally organized stress fibers when stationary. Stress fibers are also common of normal RPEs in situ as well as in immobile, adherent cells in vitro (Grierson et al., 1994). Cytokeratin expressions also change dramatically during morphological changes. In situ, RPE cells express K8 but lack larger forms such as K18 and K19, yet these are expressed by migratory fibroblastic RPEs (Grierson et al., 1994).

Substrate seems to be a crucial role in determining the morphological response of RPEs. The macrophage phenotype has been shown to develop when placed in an aqueous environment, photoreceptor debris, in the subretinal space or on loose collagen fibrils in the vitreous (Grierson et al., 1994). The fibroblast form typically generates on two-dimensional surfaces at low densities and when the surface is rich in fibronectin. This is known from studies of epidermal repair where fibronectin is abundant while cells are migratory, whereas laminin becomes the dominant protein after repair. Under crowding conditions and in the absence of fibronectin, an epithelioid type is readily adopted (Grierson et al., 1994). Maintenance of polarization also seems to be reinforced by metabolite transport and phagocytosis. Retinoids may also have growth-inhibiting properties as well as indirect influence on cytoskeletal structure (Grierson et al., 1994).

\section{Bruch's Membrane}

The Bruch's membrane is an integral part of the eye. This cellular basement membrane is a pentalaminar structure composed of a central elastin layer with 
collagenous zones on both sides, forming a $2-4 \mu \mathrm{m}$ thick boundary layer between the RPE layer and the choroid (Lee et al., 2006). Its main function is to transport nutrients from the blood supply to the RPE layer and allow for removal of waste products back to the choricocapillaris. This layer also plays an integral part in maintaining the bloodretinal boundary and increasing the stability of the neighboring layers (Aisenbrey et al., 2006).

In general, the Bruch's membrane is predominantly composed of glycoproteins, much like most basement membranes. The overall glycoprotein composition of the membrane includes collagen I and IV, laminin, fibronectin and elastin (Marshal et al., 1998). This combination of glycoproteins also plays in integral role in the signaling and polarization of the RPE layer (Grierson et al., 1994). The Bruch's membrane is also ultrastructurally and biochemically similar to other membrane complexes such as the glomerulus, lung alveoli and other endothelial-epithelial juxtapositions (Sheridan et al., 2004).

The surface of the Bruch's membrane closest to the RPE layer is known as the RPE basal lamina. This layer functions as the attachment surface for the RPE layer (Del Priore, Tezel and Kaplan, 2006). Removal of the basal lamina and the inner collagenous layer from the Bruch's membrane due to disease or damage has been shown to prevent re-epithelialization by RPEs, making resurfacing of aged Bruch's membranes difficult (Del Priore, Tezel, Kaplan, 2006).

It is at the Bruch's membrane that the introduction of multiple factors such as drusen accumulation and vascularization begins to occur, causing blurred and distorted vision. This indicates the initiation of a disease referred to as macular degeneration. 


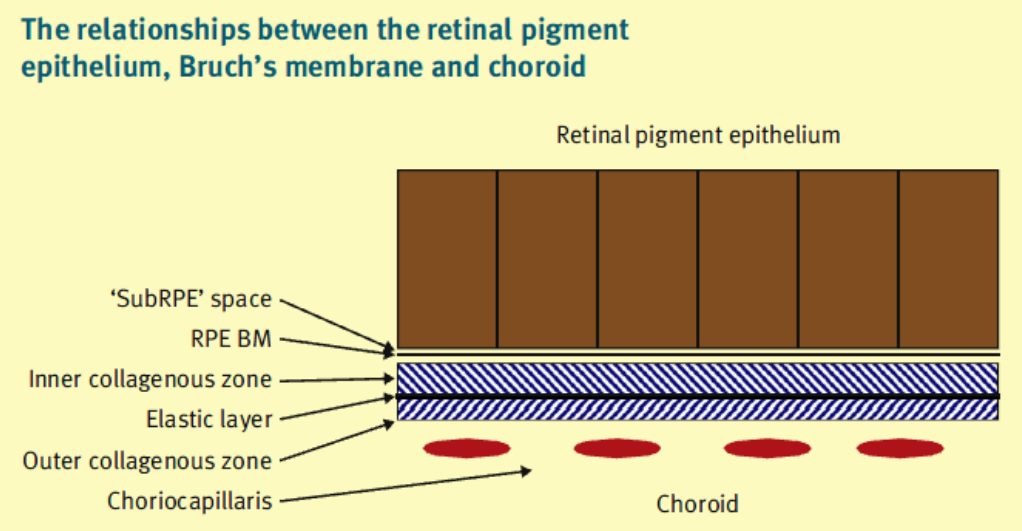

FIGURE 2- Bruch's Membrane in relation to RPEs and choroid (Luthert 2010)

\section{B. Macular Degeneration}

Age-related macular degeneration is one of the most significant causes of central vision loss and blindness in industrialized countries (Ma et al., 2009). Although this disease has been studied extensively, some of the pathological changes associated with initiating the disease are still not fully understood. The disease in general causes central vision loss related to aberrations between the RPE and the Bruch's membrane. This loss in central vision is due to progression of the disease within the macula lutea. Diagnosis of the disease is usually conducted using a visual acuity test such as an Amsler grid (Doheny Eye Institute, 2007). Missing lines, distortions or blurred vision when using an Amsler grid can be indicators of AMD. 

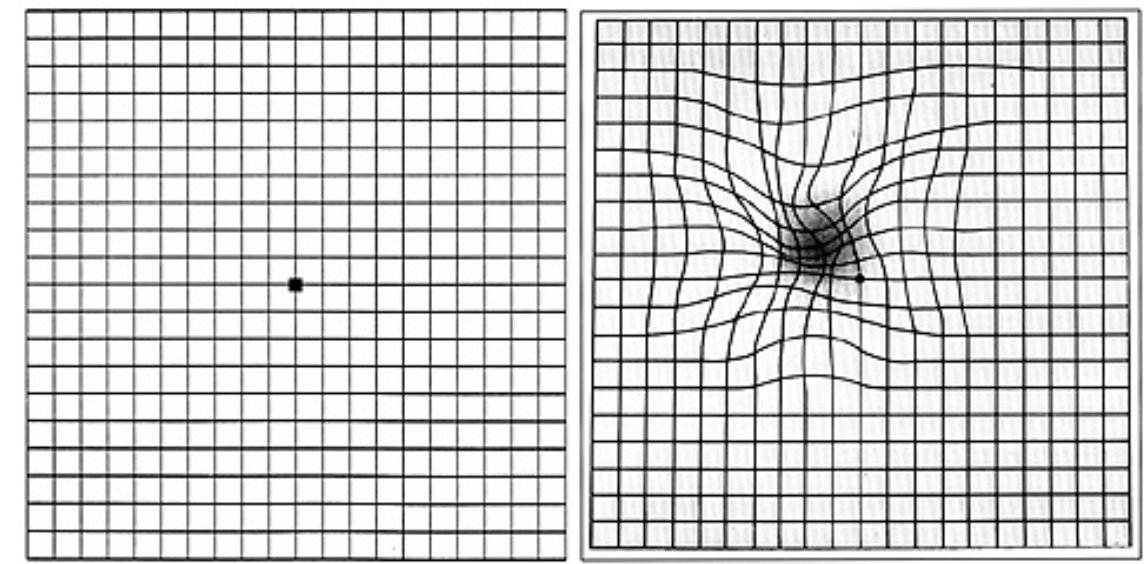

FIGURE 3- Amsler grids. Left photo indicates normal vision while the right simulates AMD. (National Eye Institute 2009)

\section{Pathology}

There are two predominant forms of AMD; the wet and dry form. The wet form of AMD, also known as exudative AMD, consists of formation of new blood vessels between RPEs and the Bruch's membrane and a loss of RPE cells (Lee et al., 2006). This is known as choroidal neovascularization (CNV). CNV is thought to be the cause of $90 \%$ of the visual impairment tied to AMD (Del Priore et al., 2006) and leads to scarring of the retina, preventing re-epithelialization by the RPE. Dry AMD, known as geographic atrophy, is linked to the natural thinning of the macula over time (Luthert et al., 2010).

When dysfunction of the RPE layer begins, degradation of photoreceptor waste products may begin to decrease, leading to drusen accumulation (Zarbin et al., 1998). Both drusen and CNV separate the basal lamina from the inner collagenous layers, distorting the RPE and photoreceptor layers above, causing visual impairment (Del Priore, Tezel, Kaplan, 2006). This increase in the Bruch's membrane thickness leads to 
a reduction in permeability, further inhibiting metabolite removal and nutrient diffusion (Lee, Vroom, Fishman and Bent, 2006). Drusen formation may also increase the risk and progression of CNV (Del Priore, Tezel, Kaplan, 2006). This drusen formation is often found in the inner collagenous layer as well as the basal lamina of the Bruch's membrane (Del Priore, Tezel, Kaplan, 2006).

Drusen are categorized by size and shape (Mecklenburg and Schraermeyer, 2007). Hard drusen typically less than $125 \mu \mathrm{m}$ wide and are well defined; soft drusen is typically more diffuse, less defined visually and carry a higher risk of progression of AMD (Luthert et al., 2010). Hard drusen appear to contain a dendritic cell process, have well defined edges and appear more frequently between the choricocapillaris and capillaries. Due to use of conflicting terminology in past publications concerning diffuse deposits, more recent categorizations based on location have been created. These categories are based on deposits internal to the RPE basement membrane laminar deposit (BlamD) and those external to the RPE basement membrane linear deposit (BlinD). BlamD contains abundant 'long-spaced collagen' similar to type VI collagen while BlinD contains more membranous material (Luthert et al., 2010).

Growth factors may also play an important role in the progression (and potential inhibition) of AMD. Pigment epithelium-derived factor (PEDF) and Thrombospondin-1 (TSP-1) are produced by highly differentiated RPEs and are potent inhibitors of angiogenesis (Ohno-Matsui et al., 2001) (Miyajima-Uchida et al., 2000). Vascular endothelial growth factor (VEGF), a pro-angiogenic growth factor, has also been shown to be produced by RPE cells on their basal side in vivo (Blaauwgeers et al., 1999). With RPEs and the Bruch's membrane in their natural forms, secretions of these growth 
factors allow for physiological maintenance of the choroid. Damage such as oxidative stress may cause overexpression of growth factors by the RPEs, causing a proinflammatory response and a destruction of the Bruch's membrane (Koh, 2000).

Hypoxia may also play a role in the development of CNV. Hypoxia markedly increases VEGF secretion, increasing the drive of CNV and causing a positive feedback mechanism (Blaauwgeers et al., 1999). A decrease in Bruch's membrane permeability due to age or drusen accumulation may also decrease the concentration of VEGF exposed to the choricocapillaris, resulting in vascular atrophy (Schlingemann et al., 2004).

Proper diagnosis can also become a complication for early stages of AMD, as it can be difficult to distinguish normal aging and pathologically significant physiological changes. Differences between normal ECM debris and drusen can be difficult to differentiate between (Del Priore et al., 2006). Normal physiological changes related to aging such as increasing thickness, deposition of ECM compounds and protein crosslinking may be indistinguishable from early stages of AMD (Gullapalli et al., 2005). On order to appropriately diagnose early AMD, further research must occur in order to accurately distinguish the disease state from the normal aging process.

\section{Current Treatments}

Relatively few treatments have been proven effective for treating wet AMD. One of the most commonly used is that of anti-VEGF treatments. Of these, ranibizumab, bevacizumab and aflibercept are the most common and approved by the FDA. 
Ranibizumab, also known as Lucentis, is commonly used for treatment of wet AMD, diabetic macular edema and macular edema following retinal vein occlusion. Ranibizumab is a monoclonal antibody fragment that binds to the receptor binding domain of all isoforms of VEGF-A (Browning et al., 2012). During clinical trials, ninety-four percent of patients maintained their current vision while almost thirty-four percent experienced an increase in visual acuity within one year given monthly doses (Rosenfield et al., 2006). Bevacizumab, also known as Avastin, is also a monoclonal antibody similar to ranibizumab, but uses a full-length antibody instead (Browning et al., 2012). Aflibercept, known as Eylea, is a newer anti-VEGF drug to come to the market which specifically binds to VEGF-A, VEGF-B and placental growth factors 1 and 2 and is reported to have a higher binding affinity than both ranibizumab and bevacizumab (Browning et al., 2012).

Another treatment for exudative AMD is thermal laser coagulation, which only restricts the progression of the disease rather than treating it (Del Priore et al., 2006). Unfortunately, this treatment also causes undesirable side effects such as scotoma due to destruction of the retinal tissue around the treatment area. Also, using this treatment method does not prevent further CNV from occurring in a large number of patients (Del Priore et al., 2006). Brachytherapy in combination with anti-VEGF medications have also been studied (Avila et al., 2009). This treatment used a single strontium-90 radiation treatment along with two injections of bevacizumab and was able to obtain similar results as monthly doses of anti-VEGF medications alone (Avila et al., 2009). Side effects for this treatment included CNV leakage in one fifth of patients as well as cataracts in a quarter of patients. In general, all of these treatments are used to prevent 
progression of the disease. Further research in the development and progression of the disease must be done in order to develop a restorative treatment.

In addition to treatments for AMD, education on potential preventative measures is highly important, especially for those that may have a significant risk of developing the disease. Many dietary supplements such as lutein, vitamins C and E, zinc, glutathione and zeaxanthin are thought to be helpful in preventing early onset of CNV associated with AMD (Nowak et al., 2006). Smokers have also been found with significantly less macular pigment, leading to a risk increase by a factor of two to three times (de Jong et al., 2006). Omega-3 fatty acid intake has also been associated with a lower risk of AMD (Ambati et al., 2012). Even though taking these supplements may help reduce the risk of developing the disease, none of these treatments are proven to prevent the disease.

In developing restorative treatments for the disease, multiple maculoplasty techniques have been tested to reintroduce functional RPEs to an excised or repaired section of Bruch's membrane. These techniques use either transplantation, translocation, stimulation or cell proliferation to repair the damaged membrane (Del Priore et al., 2006). Submacular surgeries including membranectomies have shown some progress in past studies, but RPEs often showed incomplete resurfacing as well as choricocapillaris atrophy in areas absent of RPE.

Currently, there have been limited successes with direct injection or transplantation of RPE sheets into the eye to repair damaged Bruch's membranes (Binder et al., 2004). Often after transplantation, multilayered sheets of RPE cells have formed, developing an improper orientation of cells with respect to the photoreceptors. 
Even with proper orientation, RPE adhesion may be lacking (Del Priore and Tezel, 1998). Patients must also be immunosuppressed to allow for allogenic cellular grafting (Jiang and Jorquera, 1994). Patients with subfoveal membranectomies and transplantations of adult human RPEs, even with immunosuppression, have had limited success (Kaplan and Del Priore, 1998).

RPE monolayers have also been suggested for transplantation into excised areas. By using temperature responsive materials, sheets of confluent RPE cells were obtained (Kubota et al., 2006). Due to the thin nature of the material, most sheets obtained some damage during removal from culture. Although this study proves the feasibility of generating these cell sheets, further research is still needed on creating more robust and transplantable sheets while maintaining viability and function in vivo.

Biodegradable polymers have also been proposed as a replacement for damaged Bruch's membrane. Materials such as poly(lactic acid) (PLA) and poly(DL-lactic-coglycolic acid) (PLGA) have been proposed as suitable membrane replacements due to their biocompatibility and degradability (Lu et al., 2001). In theory, cells would be seeded onto thin sheets of the material in vitro and allowed to reach confluency. The sheet would then be loaded into a micropipette and carefully injected into the damaged area. Cells would then attach themselves to the existing Bruch's membrane after degradation of the material at two to three weeks (Lu et al., 2001). Due to the fragile nature of the RPE monolayer, it may be difficult to transplant a complete monolayer of these cells using this technique. This technique also assumes that the remaining RPE layer would inherently attach to the remaining Bruch's membrane, although previous studies have not shown much success (Del Priore et al., 2006) (Gullapali et al., 2005). 
A variety of other materials have been suggested as replacement for Bruch's membrane such as collagen films, Bruch's membranes from cadavers, anterior lens capsule, Descemet's membrane and amniotic membranes (Lee et al, 2007). Although many different substrates have been proposed, no material studied so far has been able to successfully produce a fully functioning membrane for replacement.

\section{Proposed Solution}

In developing new treatments for AMD, it is important to understand that the replacement Bruch's membrane must be of comparable thickness, similar permeability and provide an effective cellular scaffold in which to support the native RPE layer (Lee

et al., 2006). The material must also be a permanent replacement for the native Bruch's membrane, providing enough similar mechanical and chemical responses as to allow the RPE layer above to maintain a functioning membrane. The replacement membrane could also potentially provide chemical cues such as VEGF to sustain the choricocapillaris until a functioning RPE layer is obtained.

The concept this research is based upon focuses on the development of a permanent, synthetic Bruch's membrane. This synthetic membrane would mimic the natural functions of the Bruch's membrane such as adequate permeability for the diffusion of nutrients and metabolites, comparable mechanical strength as well as biological molecules to promote RPE adhesion and differentiation. (Del Priore et al., 2006). PEG hydrogels meet the requirements for a suitable membrane in these areas, but it is known for its poor ability for cell adhesion. In order to alleviate this problem, 
PEG gels modified with adhesion ligands on the surface layer have been developed to provide cellular adhesion to the apical side of the gel. With proper concentrations of ligands, these gels would allow for adequate adhesion of RPE cells to promote confluent coverage as well as appropriate epithelial morphology. This research is focused on obtaining an optimal concentration of surface adhesion peptides to allow for appropriate RPE development based on ZO-1, actin expression, and other factors such as cell circularity, area and actin orientation. All gel concentrations will be compared to fibronectin coated glass coverslips as a positive control while PEG only hydrogels will function as negative controls. Both ARPE-19 and PRPE cell lines will also be used in order to evaluate the differences in immortalized and primary RPE cells when seeded on these gels.

\section{C. $\quad$ Hydrogels}

\section{Hydrogel History}

Hydrogels are water-insoluble polymers which absorb a large amount of water. These polymers can be derived from both natural and synthetic materials and are used in a variety of fields (Society for Biomaterials, 2007). Hydrogels have been used in biomedical disciplines for over fifty years in areas such as ophthalmology and surgery (Gibas et al., 2010). The first hydrogel developed for potential biomedical use was poly-2-hydroxyethyl methacrylate, originally used for soft contact lenses (Gibas et al., 2010). In the 1980s, calcium alginate was developed for creating microcapsules for 
cellular engineering (Gibas et al., 2010). Hydrogels today are often used in prevention of thrombosis, drug delivery, post-operative adhesion, biosensor coating, cell scaffolds and transplantation (Gibas et al., 2010)(Brandl et al., 2007).

In order to absorb large quantities of water without dissolving, hydrogels are crosslinked using chemical or physical means. This allows the material to maintain integrity when placed in a solution. In order to develop these crosslinks, hydrogels are commonly bound together using cross-linking agents such as succinimidyl ester amine modification or using free radical polymerization. One common technique used to create hydrogels in situ is photopolymerization, which uses a light to generate a free radical reaction with a crosslinking reagent to combine two polymers. Use of this technique allows spatial control, curing speed control and minimal heat production (Gibas et al., 2010).

\section{Poly(ethylene glycol) Hydrogels}

PEG hydrogels are well known for their biocompatibility and optical transparency (Brandl et al., 2007). Their ability to form semi-permeable membranes and large aqueous content make them a strong candidate for a synthetic Bruch's membrane. Other factors such as mechanical stiffness and functional modification also help promote their use in biomedical applications (Brandl et al., 2007). This versatility of PEG is made possible by first functionalizing the ends of the PEG hydroxyl chains in order to allow them to bind to one another (if the original hydroxyl groups are not used). Both ends of the chain can be either purchased or synthesized with the same or 
different functional groups to allow for further control of how the polymer crosslinks to other chains.

There is only one caveat to the use of PEG hydrogels for the use of a synthetic Bruch's membrane; its inability to promote cellular adhesion (Gombotz, 2004). This can be resolved by incorporating functional PEG groups that promote cellular adhesion.

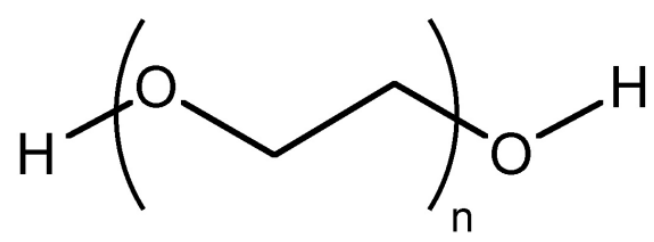

FIGURE 4 - Poly(ethylene glycol) (Ozden 2012)

An excellent example of using different functional groups with this polymer are ligand modified PEG polymers. The use of modified PEG hydrogels with attachment ligands is very well documented (Patel et al., 2005). Ligands such as YIGSR and RGD(S), peptide segments of laminin and fibronectin, respectively, are commonly used to promote cellular adhesion to materials. These PEG-peptide polymers have been used with multiple cell types such as smooth muscle cells and preadipocytes (Mann et al., 2001) (Patel et al., 2005). Attachment and migration has also been studied using fibroblasts on modified PEG hydrogels (Gobin and West, 2002). Using specific attachment ligands also promotes specific cellular adhesion (Mann et al., 2005). Specific placement of these attachment molecules also add a spatial component to the synthesized gel, allowing for specific cell targets to bind in certain areas while preventing adhesion in another. 


\section{Adhesive Ligands}

Many cell types are known to use different adhesion ligands in order to interact with the space around them. Integrins and laminins are often used as cues to promote cellular attachment (Aisenbrey et al., 2006). Integrins are trans-membrane proteins that specifically function as adhesion receptors for cells (Takada et al., 2007). These receptors bind to ligands in the ECM, a cell surface or in the extracellular solute (Takada et al., 2007). Ligands are heterodimeric and are composed of an $\alpha$-subunit and a $\beta$-subunit (Aisenbrey et al., 2006). There are currently eighteen known $\alpha$ subunits and eight $\beta$-subunits, forming twenty-four unique combinations that have characterized so far (Takada et al., 2007). For RPEs, integrins $\alpha 3 \beta 1, \alpha 6 \beta 1$ and $\alpha 6 \beta 4$ have significant interactions regarding cellular attachment (Aisenbrey et al., 2006). Commonly, integrins will interact with a variety of extracellular proteins, such as fibronectin and laminin (Boateng et al., 2004).

With respect to RPE cells, it has been found that adding adhesion ligands such as laminin, fibronectin and vitronectin to aged Bruch's membranes promote attachment when compared to non-treated membranes (Tezel, Del Priore and Kaplan, 2004). This proves that in order to appropriately replace functional RPE cells, a layer of attachment ligands is required. Previous studies have also shown that RPE cells will adequately adhere to coatings of fibronectin as well as PEG-RGDS hydrogels (Scherzer ,2011). Although PEG-RGDS has been proven as an adhesion ligand, it is possible that it may alter cellular function and morphology (Boateng et al., 2004). In order to unveil whether or not this is the case with RPE cells, this research is focused 
on observing morphological properties such as cell circularity, ZO-1 expression and actin filament length to ascertain if RPE cells remain differentiated.

\section{RGDS}

RGDS, the amino acid sequence Arg-Gly-Asp-Ser, is a well-known peptide found in the tenth domain of fibronectin and is highly involved in cellular attachment (DeLong et al., 2005). The modification of PEG hydrogels with this peptide sequence has also been studied extensively with human dermal fibroblasts (Gobin and West, 2002)(Hahn et al., 2006) as well as with RPE cells (Scherzer, 2010). RGDS immobilized films have also been shown to be more stable against heat treatment and enhance cellular growth better than fibronectin films (Ito et al., 1991). Some sources are commonly found referring to the RGD sequence in respect to cellular attachment. Studies have found that when using three different versions of RGD- RGDS, RGDV and RGDT, the RGDS was found significant in cellular attachment across five different cell tpyes (Hirano et al., 1993).

RGDS has also been shown to stimulate TGF- $\beta 1$ sectetion in human mesangial cells when bound to integrins (Ortega-Velazquez et al., 2003). TGF- $\beta 1$ has also been linked to proliferation of RPE cells via epithelial-mesenchymal transition (Li et al., 2011). This shows that using RGDS as an attachment ligand will promote RPE proliferation into non-confluent sections of a coated gel.

The research herein will determine whether or not varied concentrations of PEG$\operatorname{RGDS}(0,5,10$ and $20 \mu \mathrm{mol} / \mathrm{mL})$ applied during the gel coating process will affect the 
deposited surface concentration on PEG hydrogels. Each concentration will then be analyzed using phase contrast and confocal microscopy for differences in cellular attachment and morphology to determine if an optimal concentration of PEG-RGDS can be applied to encourage appropriate RPE expression. 


\section{METHODS AND MATERIALS}

In this study, poly(ethylene glycol) (PEG) hydrogels were used as the substrate material to mimic the Bruch's membrane. PEG hydrogels were modified with different concentrations $(0,5,10$ and $20 \mu \mathrm{mol} / \mathrm{ml})$ of RGDS adhesion peptides. The effectiveness of cell adhesion and confluency was measured using both optical microscopy and confocal microscopy techniques. To determine morphological differences, ZO-1 and actin were stained and imaged via fluorescence and immunofluorescence using confocal microscopy. The step-by-step procedures and protocols used to fabricate these materials, culture the cells on the biomimetic materials and image these substrates is presented in detail below.

\section{A. $\quad$ Cell Culture}

Previous studies have indicated that immortalized cells may behave differently than primary lines, especially in RPE cells (Ablonzsky et al., 2011). These differences can be observed in a multitude of ways, such as the morphological distribution of ZO-1 and actin. In order to determine the effects of RPE cell type when using PEG-RGDS coated hydrogels, both immortalized ARPE-19 and primary PRPE cells were used for this study. 


\section{1. $\underline{\text { ARPE-19 }}$}

Adult retinal pigmented epithelial cells (ARPE-19, Retinal Epithelium, Human; ATCC; Manassas, VA; Item \# CRL-2302) are a spontaneously immortalized cell line created by Dunn et al. (Dunn et al., 1996). Cells were incubated at $37^{\circ} \mathrm{C}$ with $5 \% \mathrm{CO}_{2}$ and cultured in T-75 culture flasks $\left(75 \mathrm{~cm}^{2}\right.$ Cell Culture Flask; Tissue Culture Treated with Vent Cap; Corning Inc.; Item \# 430641) using Dulbecco’s Modified Eagle Medium: Nutrient Mixture F-12 (DMEM/F-12; Corning Inc; Item \# 10-092-CV) containing 10\% fetal bovine serum (FBS; Gibco; Invitrogen; Item \# 26140-079) and 1\% Penicillin, Streptomycin and L-Glutamine (PSG; 10,000 IU/mL penicillin, 10,000 $\mathrm{gg} / \mathrm{mL}$ streptomycin, 29.2mg/mL glutamine; Cellgro; Mediatech; Item \# 30-009CI) and fed $12 \mathrm{~mL}$ three times a week. This media along with bovine serum was chosen in order to encourage proliferation of cells, which has been successfully used by other labs in culturing this cell type (Aisenbrey et al., 2006).

Once plated, cells were allowed to grow a minimum of two weeks postconfluency in order to obtain adequate cell densities with a majority of epithelioid phenotype. To passage, Trypsin-EDTA (Trypsin-ethylene diamine tetra acetic acid; Cellgro; Mediatech, Inc.; Item No. 25-052-Cl) was heated in a water bath at $37^{\circ} \mathrm{C}$ then $2 \mathrm{~mL}$ were added per T-75 culture flask after first aspirating the used media and rinsing once with PBS. The flask was then allowed to incubate for 10 minutes. After the cells detached from the flask, $5 \mathrm{~mL}$ of media was added to deactivate any residual trypsin and the cell suspension was moved into a $15 \mathrm{~mL}$ centrifuge tube. The centrifuge tube was then centrifuged for ten minutes at $1000 \mathrm{rpm}$ (Beckman Coulter Allegra 6R 
Centrifuge). Residual media was aspirated from the cell pellet and the cells are resuspended in media. Cells were typically passaged at 1:6 and passages 11-12 were used for these studies, although passage numbers for immortalized cell lines are insignificant since they do not change characteristically between passages.

\section{PRPE}

Porcine retinal pigmented epithelial cells (PRPE) were obtained from primary cultures from Qun Zeng, a postdoctoral researcher from the Tezel Lab in the Department of Ophthalmology and Visual Sciences. This cell type was cultured under similar conditions as ARPE-19 cells. However, the primary cultures were only passaged once to ensure adequate ZO-1 expression and decrease risk of dedifferentiation prior to seeding on gels. Cells were allowed to grow a minimum of one-week post-confluency to ensure proper epithelioid phenotype.

\section{B. PEG-DA Synthesis}

Poly(ethylene glycol) (PEG) (MW 6000; EMD; Item \# PX1286L-4) was weighed out in a glass beaker and then lyophilized overnight to remove any water from the sample. All necessary glassware was dried overnight at $100^{\circ} \mathrm{C}$. Synthesis begins by adding PEG to a round bottom flask thrice evacuated and filled with argon. The flask was then evacuated and filled again with argon. Up to $40 \mathrm{~mL}$ of anhydrous dichloromethane (DCM; Sigma; Item \# 270997) was added $10 \mathrm{~mL}$ at a time via glass 
syringe. Enough anhydrous DCM was used to make a non-viscous solution. The evacuation process was once again repeated with argon. Triethyl amine (TEA; Sigma; Item \# 90335) was then added to the solution at a 2:1 molar ratio and allowed to mix for five minutes. TEA acts as a catalyst by binding to free hydroxyl groups. After repeating the evacuation process, acryloyl chloride (Sigma, Item \# A24109) was added slowly to the flask at a 2:1 molar ratio. Acryloyl chloride reacts with bound TEA on PEG to create acrylate end groups. The solution was then evacuated with argon and allowed to react overnight.

After allowing the mixture to react for at least 12 hours, the phase separation process begins. The PEG mixture was moved into a $500 \mathrm{~mL}$ separatory funnel. $2 \mathrm{M}$ potassium carbonate $\left(\mathrm{K}_{2} \mathrm{CO}_{3}\right.$; Acros; Item \#42408) was added to the mixture to wash the solution. The funnel was then sealed and shaken, venting after every fifteen to twenty seconds. This process was repeated until no more $\mathrm{CO}_{2}$ was released. Parafilm was then placed on the separatory funnel in place of the stopper and allowed to separate for 24-36 hours. This separation allows for the separation of $\mathrm{KCl}$ into the aqueous phase and the PEG-DA into the organic phase.

Next was the purification process. After allowing the organic and aqueous phase to separate, the denser organic phase was drained into a separate beaker. Magnesium sulfate $\left(\mathrm{MgSO}_{4}\right.$; Fisher; Item \#M65-500) was added as a drying agent while stirring the organic phase. The mixture was then filtered using a vacuum flask and a Buchner funnel with the filter paper pre-wet with DCM to remove the $\mathrm{MgSO}_{4}$. Diethyl ether $\left(\left(\mathrm{C}_{2} \mathrm{H}_{5}\right)_{2} \mathrm{O}\right.$; Sigma; Item \#673811) was then added to the remaining PEG solution in a 2 L beaker to precipitate the PEG-DA out of solution. The precipitate was then filtered 
using a Buchner funnel and paper pre-wet with ether. The PEG-DA powder was then dried overnight under vacuum, dialyzed overnight in a 3500 Dalton molecular weight membrane (Spectra/Por 7 Dialysis Membrane; molecular weight cutoff 3500; Spectrum Laboratories; Item \# 132111) in deionized water and sequentially lyophilized overnight. The resultant PEG-DA powder was stored under argon at $-20^{\circ} \mathrm{C}$.

\section{PEG-RGDS Synthesis}

PEG-RGDS was synthesized using a combination of monoacrylated PEG succinimidyl valerate at 3400 dalton molecular weight (Acrylate-PEG-SVA; Laysan Bio; Item \# ACRL-PEG-SVA-3400-1g.) and RGDS peptide (H-Arg-Gly-Asp-Ser-OH; Calbiochem; Item \# 03-34-0002-25MG.) at a 1:1 molar ratio. These are first dissolved separately in $50 \mathrm{mM}$ sodium bicarbonate $\left(\mathrm{NaHCO}_{3}\right.$; Mallinckrodt Chemicals; Item \# 7412-12) in deionized water at a minimum $\mathrm{pH}$ of 8.5. The dissolved RGDS was then added dropwise to the Acrylate-PEG-SVA solution and was allowed to mix on an orbital shaker in a centrifuge tube wrapped in foil for a minimum of four hours. The salt and SVA ester was removed from the solution by dialysis using a 3500 Dalton molecular weight cutoff membrane (Spectra/Por 7 Dialysis Membrane; molecular weight cutoff 3500; Spectrum Laboratories; Item \# 132111). The residual PEG-RGDS solution was then lyophilized overnight. The resultant PEG-RGDS powder was stored under argon at $-20^{\circ} \mathrm{C}$. 


\section{D. $\quad$ PEG-RGDS-Fluor 488 Synthesis}

PEG-RGDS-Fluor 488 was synthesized in a method similar to PEG-RGDS. PEGRGDS was either allowed to conjugate in the sodium bicarbonate solution as explained above, or was added back into sodium bicarbonate solution of the same strength (50 mM, pH 8.5 minimum). AlexaFluor 488 carboxylic acid, succinimidyl ester (AlexaFluor 488 carboxylic acid, succinimidyl ester; Invitrogen; Item \# A20000) was then added dropwise to the PEG-RGDS solution at a molar concentration of 10:1 (AlexaFluor 488:PEG-RGDS). The mixture was allowed to react for a minimum of four hours on an orbital shaker while wrapped in foil. The salt and residual AlexaFluor 488 dye was removed from the solution by dialysis using a 3500 Dalton molecular weight cutoff membrane (Spectra/Por 7 Dialysis Membrane; molecular weight cutoff 3500; Spectrum Laboratories; Item \# 132111). The residual PEG-RGDS-Fluor 488 solution was then lyophilized overnight. The resultant PEG-RGDS-Fluor 488 powder was stored under argon at $-20^{\circ} \mathrm{C}$ wrapped in foil.

\section{E. PEG-DA Hydrogel Formation}

Molds for gels are preassembled using glass coverslides $(25 \mathrm{~mm}$ x $75 \mathrm{~mm} \times 1 \mathrm{~mm}$; VWR; Item \# 48300-025), 0.5 mm electrophoresis spacers (Fisher; Item \# FBVE1033) and binder clips (Staples, Item \# 831610). Silicone vacuum grease (Dow Corning; Sigma; Item \# Z273554-1EA) was used to seal the mold and the molds are sterilized overnight using a UV light. PEG-DA hydrogels are polymerized using 10\% w/v PEG- 
DA in (10mM) HEPES. Twenty microliters of acetophenone photoinitiator (2, 2dimethoxy-phenyl-acetophenone; Sigma; $196118-50 \mathrm{G})$ at $200 \mathrm{mg} / \mathrm{mL}$ in Nvinylpyrrolidone (NVP; Acros Organics; CAS \# 88-12-0) was added per milliliter of PED-DA solution. The solution was then filter-sterilized using a $0.22 \mu \mathrm{m}$ PES syringe filter (Millex-GP; Millipore Corporation; Item \# SLGP033RS) in the biological laminar flow hood. Approximately $1.5 \mathrm{~mL}$ of PEG-DA solution was then injected into each mold and was polymerized for 1 minute using a 5mW 365nm UV light (Blak-Ray B100-A; Fisher). The molds are then opened to expose the gel sheets and circular gels are punched using a \#5 (10mm) hole punch. Four gels were prepared for each peptide concentration group $(0,5,10$ and $20 \mu \mathrm{mol} / \mathrm{mL})$ described below in the next section.

Gels are then allowed to swell in PBS for a minimum of one hour at room temperature.

\section{F. $\quad$ Hydrogel Coating Procedure}

PEG-DA hydrogels are surface modified with PEG-RGDS using either 5, 10 or $20 \mu \mathrm{mol} / \mathrm{mL}$ solution concentrations of PEG-RGDS. These solutions are made by weighing out the desired amount of PEG-RGDS for a $20 \mu \mathrm{mol} / \mathrm{mL}$ solution and adding the powder to $10 \mathrm{mM}$ HEPES. Enough solution was made to serial dilute and create the other required concentrations. Photoinitiator was then added at a ratio of $20 \mu \mathrm{L}$ per $\mathrm{mL}$ of PEG-RGDS solution. The solution was then filter-sterilized using a $0.22 \mu \mathrm{m}$ PES syringe filter (Millex-GP; Millipore Corporation; Item \# SLGP033RS) in the biological laminar flow hood. Non-coated gels are prepared by placing the gels to be coated onto coverslides and removing any residual PBS. Ten microliters of the desired 
concentration of PEG-RGDS is then added to the top of each gel and is covered using a 24 x 60 mm coverslip (VWR; Item \# 48393-106). This was determined based on the amount of solution required to completely coat the surface of the gel. The gels are then polymerized for 3 minutes using a 5mW 365nm UV light (Blak-Ray B100-A; Fisher). Each gel was then placed into a 24-well culture plate (Corning Costar; Sigma; Item \# CLS3524-100EA) and rinsed with $500 \mu \mathrm{L}$ of PBS.

\section{G. Quantification of Peptide Coating Using Rinse Supernatant}

After PEG-DA hydrogels are surface modified with PEG-RGDS-Fluor 488 using the same technique as described in section $\mathrm{F}$ above. In this instance, gels are coated with a 10:1 ratio of PEG-RGDS to PEG-RGDS-Fluor 488 using the same total concentrations $(20,10$ and $5 \mu \mathrm{mol} / \mathrm{mL})$. These gels are polymerized individually in 12 well plates and covered with a $12 \mathrm{~mm}$ coverslip to ensure a flat coating (Fisher; Item \# 12-545-80). After polymerization and the initial PBS rinse of $500 \mu \mathrm{L}$, each gel was rinsed twice at 4 and 24 hours after polymerization to remove any non-grafted peptide conjugate. All three rinses are collected cumulatively from each individual gel.

PEG-RGDS-Fluor488 coating fluorescence standards are also made by adding $5 \mu \mathrm{L}$ of each coating concentration in wells of a 24 -well plate, covering with a $12 \mathrm{~mm}$ coverslip and exposing to UV along with the coated gels. $750 \mu \mathrm{L}$ of PBS was then added to each standard to equal $100 \%$ coating solution concentrations for fluorimetric standards.Individual sample wells and coating standards were measured using a fluorimeter (Turner Biosystems Modulus 9200). These values were then compared to 
previously obtained fluorescent standards for concentrations to determine coating concentration values. Average percent grafted, concentration grafted and $\mu \mathrm{g} / \mathrm{cm}^{2}$ was calculated for each group. Average percent grafted was calculated by subtracting the average rinse concentration from the average coating concentration. Total concentration grafted was then calculated using the measured coating concentration multiplied by the percent grafted to the gel. The concentration grafted was then translated into micrograms grafted to the gel surface using the coating volume and the molecular weight of the peptide. This was then multiplied by the surface area of the gel to determine the average $\mu \mathrm{g} / \mathrm{cm}^{2}$ per group. These values were recorded in Microsoft Excel 2012 and statistically analyzed by a paired t-test using Minitab 16 statistical software and $\alpha=0.05$.

\section{H. Quantification of Peptide Coating using Confocal Microscopy}

Fluorescently coated gels are imaged using Nikon Eclipse Ti (Nikon Instruments Inc.; Melville, NY) running the Nikon Elements Image Software (NIS-Elements, AR 3.1.Ink; Laboratory Imaging, Nikon Instruments Inc.; Melville, NY). Images were taken using a 60x oil immersion objective. The basic confocal setting are as follows: image capturing speed of 1/8 frames/sec, an image size of 2048x2048 pixels, and a pinhole size $24.3 \mu \mathrm{m}$. The initial GFP-UV (for AlexaFluor 488) settings were HV=120, offset $=0$ and laser power $=5 \%$ and the Z-steps were set at $1 \mu \mathrm{m}$ with a total area of 20 $\mu \mathrm{m}$, creating 21 images. Z-stacks were then exported into separate TIF files for each Zstack acquired. Individual images were then combined using ImageJ (Version 1.47) into 
stacks and converted into maximum intensity images to display the total maximum intensity per sample. All images were then set to a unified scale of 200-2000. The average intensity of each image was then recorded using the 'Analyze -> Measure' feature. These values were recorded in Microsoft Excel 2012 and statistically analyzed by a paired t-test using Minitab 16 statistical software and $\alpha=0.05$.

\section{I. $\quad$ Preparation of RPEs on Hydrogels}

Surface modified PEG-DA hydrogels are seeded with cells at the appropriate cellular density to achieve confluency for each of the respective cell types and gel groups (ARPE-19 were approximately 90,000 cells/cm², PRPE were approximately 110,000 cells $/ \mathrm{cm}^{2}$ ) and were dry seeded by adding cell suspension onto the top of gels in 12-well plates. After allowing 4 hours for cellular adhesion to the surface of the gel, the wells were flooded with media to the required volume $(500 \mathrm{~mL})$. A minimum of four gels were prepared for each group $(0,5,10$ and $20 \mu \mathrm{mol} / \mathrm{mL})$ as well as for each cell type. Gels were then held stationary using size M7 A2 stainless steel washers (Grainger, Item \# 6EY95) and $500 \mu \mathrm{L}$ of DMEM/F-12 media was added to each gel. The cells on the gels were fed three times a week. After seven days, the cell-coated gels were rinsed briefly with PBS and fixed with 4\% formaldehyde (16\% Formaldehyde diluted in PBS; Thermo Scientific; Item \#28908) for 10 minutes. 


\section{J. $\quad$ Preparation of RPEs on Fibronectin}

Fibronectin coated coverslips were also used in each study as a positive control. The control samples were constructed by coating $12 \mathrm{~mm}$ glass coverslips with fibronectin (Sigma; Item \# F1141) at $1.825 \mu \mathrm{g} / \mathrm{cm}^{2}(100 \mu 1$ of $21.25 \mu \mathrm{g} / \mathrm{mL}$ per coverslip). The coated coverslips were then left at $4{ }^{\circ} \mathrm{C}$ overnight in the refrigerator and the residual liquid coating was removed before seeding. Cells were then dry seeded at the appropriate cellular density to achieve confluency for 4 hours and then fed DMEM/F-12 three times a week. After seven days, the clips were rinsed briefly with PBS and fixed with 4\% formaldehyde (16\% Formaldehyde diluted in PBS; Thermo Scientific; Item \#28908) for 10 minutes.

\section{K. $\quad$ Phase Contrast Microscopy}

Fixed gels and coverslip controls were imaged to determine cell confluency using a phase contrast microscope (Nikon Eclipse TE2000-4) equipped with a Retiga 2000R Fast Cooled Mono 12 Bit camera (Q Imaging) and interfaced with Metamorph software (v7.0r2; Universal Imaging Corporation). These images were taken using a 10x air objective. Light intensity and exposure were adjusted until cell boundaries were easily visible. Images were then saved as TIF files and cropped to $1200 \times 1200$ to remove objective artifacts shown in the corners of original saved images. Images were also optimized with the ImageJ software using the 'Process -> Enhance Contrast' setting with saturated pixels set at $0.4 \%$ to allow for a more defined boundary layer on each 
image. Next, using the 'Freehand Selections' tool, cell edges exposing the underlying gel were traced and filled in with a solid black color using 'Edit $->$ Fill'. After covering all exposed areas of the gel, a threshold image was generated using 'Image -> Adjust -> Threshold' to convert the image into a binary state. Cells were white while the exposed gel area was black. The number of white pixels for each image was then obtained using 'Analyze -> Histogram' and selecting the 'List' button to present numerical values. This list was then saved and opened in Excel. Using the ratio of white pixels to the total number of pixels per image, a percentage of total confluency was obtained. The obtained percentages for each image were then statistically analyzed using a paired ttest using Minitab 16 statistical software and $\alpha=0.05$.

\section{L. $\quad$ Immunostaining Procedure}

After 7 days, samples were placed in 12-well plates, permeabilized and blocked with $0.5 \%$ Triton-X (Triton X-100; Item \# 082513) in PBS with 1\% bovine serum albumin (BSA, Sigma, Item \# A7906-10G) and 0.2\% sodium azide $\left(\mathrm{NaN}_{3}\right.$, Acros Organics, Item \# 190380050) (PBS-BSA-Azide) for 10 minutes, respectively. The samples were then placed on an orbital rocker throughout the duration of staining. The samples were then rinsed twice with $300 \mu \mathrm{L}$ of PBS-BSA-Azide for 5 minutes each.

For each gel sample, $250 \mu \mathrm{L}$ of staining solution was used, except for the fibronectin control slips where $200 \mu \mathrm{L}$ of staining solution was used. Following rinsing, the ZO1primary antibody (Rabbit polyclonal Anti-ZO-1; Abcam; ab58720) was added at 1:50 
dilution. The sample was then placed on the rocker at $4{ }^{\circ} \mathrm{C}$ in the cold room overnight to allow the primary antibody ample time to bind.

The following day, samples are removed from the rocker, allowed to acclimate to room temperature and rinsed 6 times with $300 \mu \mathrm{L}$ of PBS-BSA-Azide for 5 minutes each. Next, the AlexaFluor 488 labeled secondary antibody (Goat anti-rabbit; Invitrogen; Item \# A11034) was added to each sample at 1:100 dilution in PBS-BSAAzide and rocked for 1 hour at room temperature. Following another 5 rinses with 300 $\mu \mathrm{L}$ of PBS-BSA-Azide for 5 minutes each, rhodamine-phalloidin (R-P; Invitrogen; R415) in PBS-BSA-Azide at 1:40 dilution was added to the samples and rocked for 40 mins at room temperature. The both gel groups and controls were then rinsed twice with $300 \mu \mathrm{L}$ of PBS-BSA-Azide for 5 minutes each. Lastly, the samples are stained with 4',6-diamidino-2-phenylindole (DAPI; Invitrogen; D1306) at 600nM concentration in PBS-BSA-Azide for 5 minutes. The samples were then rinsed twice with PBS-BSAAzide and ready for confocal microscopy analysis.

\section{Confocal Microscopy}

The immunostained gels and controls were imaged using the same confocal imaging configuration as mentioned above in section $\mathrm{H}$ except the basic confocal settings were as follows: image capturing speed of $1 / 24$ frames/sec, an image size of $2048 \times 2048$ pixels, and a pinhole size from 14.3-32.1. Three imaging channels were obtained; DAPI, GFP-UV, and TxRed. The initial DAPI settings were HV=84-129, Offset $=0$ and Power $=2-20.1 \%$, while the initial GFP-UV (for AlexaFluor 488) 
settings were $\mathrm{HV}=75-101$, Offset $=0$ and Power $=0.5-5.4 \%$ and the initial TxRed (for R-P) settings were HV=70-100, Offset= 0 and Power $=0.1-2.1$. Besides image size and frame speed, each of these parameters were adjusted manually in order to optimize each recorded image. Z-steps were recorded for each image in order to provide a fully encompassing image of ZO-1 and actin formations at different heights within the cell. An average of $20 \mathrm{Z}$-stack images per final image were acquired for each gel to encompass the entire thickness of each gel's cell layer. Z-stacks were then exported into separate TIF files for all three channels and into separate Z-stacks. Individual channels were then combined using ImageJ into stacks and converted into maximum intensity images as well as composite images with all color channels. Due to the significant amount of time required to image individual gels as well as the requirement to prepare each gel shortly before imaging to prevent drying, a sample size of $n=2$ was used for each set to reduce the effect of photobleaching during preparation and decrease the effect of time lapse between samples.

\section{N. ZO-1 Analysis}

Staining for ZO-1 was analyzed using ImageJ software. Maximum intensity images obtained using confocal microscopy were analyzed by first adjusting the image brightness and contrast using 'Image -> Adjust -> Brightness/Contrast' to allow for easy visualization of ZO-1 boundaries. Cell ZO-1 boundaries were then manually traced using the 'Polygon Selections' tool. Once a cell was traced completely, a line was drawn onto the image using 'Edit -> Draw'. This was repeated until no other cells 
could be accurately traced or a sufficient number of samples were obtained to represent the gel. Samples between $n=1$ and 21 were obtained for the images collected for this experiment, depending on the quality of the image and cell size. Once completed, the file was saved separate from the original ZO-1 image for later use. After the cells were traced, a binary image was generated using 'Image -> Adjust -> Threshold' to convert the image into a binary state and only display the tracings. This image was then saved as a separate file and numbered to correlate to measured values. The non-numbered tracings were then individually selected in numerical order using the 'Wand' tool. The tracings were then measured for area, perimeter, circularity and aspect ratio using 'Analyze -> Measure'. This was repeated sequentially for each tracing in order and the measurements were saved to an Excel file. After each image was analyzed, all measurements were compiled into one single Excel document and average values of area, perimeter, circularity and aspect ratio were obtained for each group. The compiled data was also pasted into Minitab 16 and statistically analyzed by a multifactor ANOVA and a non-paired t-test assuming equal variance using Minitab 16 statistical software and $\alpha=0.05$.

\section{O. Actin Analysis}

Staining for Actin was analyzed using ImageJ software. Maximum intensity images obtained using confocal microscopy were analyzed by first adjusting the image brightness and contrast using 'Image -> Adjust -> Brightness/Contrast' to allow for easy visualization of Actin filaments. Actin filaments were then manually traced using 
the 'Line' tool. Once traced, a line was drawn onto the image using 'Edit -> Draw'. This was repeated until a sufficient number of samples were obtained to represent the gel. Once completed, the file was saved separate from the original Actin image for later use. After the cells were traced, a binary image was generated using 'Image -> Adjust $>$ Threshold' to convert the image into a binary state and only display the tracings. This image was then saved as a separate file and numbered to correlate to measured values. The non-numbered tracings were then individually selected in numerical order using the 'Wand' tool. The tracings were then measured for length and angle using 'Analyze > Measure'. This was repeated sequentially for each tracing in order and the measurements were saved to an Excel file. After each image was analyzed, all measurements were compiled into one single Excel document and average values of length and orientation were obtained for each group. The compiled data was also pasted into Minitab 16 and statistically analyzed by a multifactor ANOVA and a non-paired ttest assuming equal variance using Minitab 16 statistical software and $\alpha=0.05$. 


\section{RESULTS AND DISCUSSION}

Four specific aims were addressed in this research. First, the concentration of PEG-RGDS deposited onto each gel group was evaluated and quantified using a fluorescently tagged PEG-RGDS substitute. The second specific aim was to evaluate the ability of PEG-DA hydrogels surface coated with PEG-RGDS to functionally support attachment of RPE cells. The last two specific aims focused on the observation of the morphology of both ZO-1 and actin expression by both ARPE-19 and primary porcine RPE cells. Staining for these specific cellular structures allowed for quantification of cell circularity, cell area, aspect ratio, actin length and orientation. Four gel concentration groups $(0,5,10$ and $20 \mu \mathrm{mol} / \mathrm{mL})$ were used for each study as well as fibronectin coated $12 \mathrm{~mm}$ coverslips for a positive control.

\section{A. Quantification of PEG-RGDS Coating (Fluorescence)}

Quantification of the PEG-RGDS coating applied to each group of gels was analyzed using two different techniques. The first was using retained rinses from individual gels and obtaining a fluorescent intensity measurement for the amount of PEG-RGDS-Fluor 488 not polymerized to the surface of the gel. These values were then compared to a standard generated by using the same volume of each concentration as the gel coating, exposing it to the same UV time and diluting using the same amount of rinses. Using the standard curve generated, the average 
concentration per group, percent PEG-RGDS-Fluor 488 grafted and an average concentration per centimeter squared was obtained. The results of this experiment are shown in Figures 5 as well as in Tables I-III. Calculations were obtained using the procedures described in the methods and materials section above.

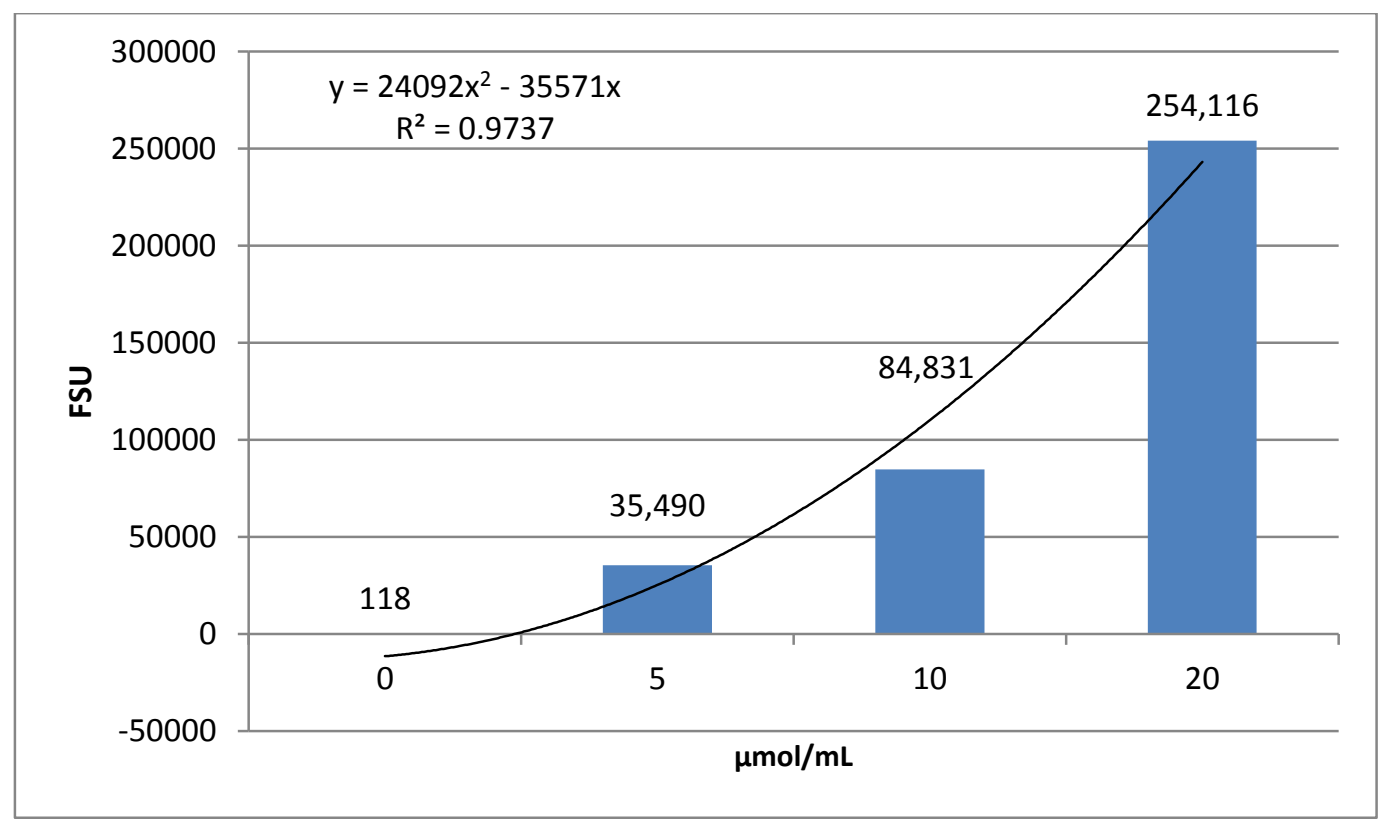

FIGURE 5- Standard Curve of PEG-RGDS-Fluor 488 Fluorescence

\begin{tabular}{|l|c|r|}
\hline $\begin{array}{c}\text { Conc. } \\
\mu \mathrm{mol} / \mathrm{ml}\end{array}$ & Avg. FSU & \multicolumn{1}{c|}{$\begin{array}{c}\text { Avg. } \\
\mu \mathrm{mol} / \mathrm{mL}\end{array}$} \\
\hline 0 & 118 & 0.026 \\
\hline 5 & 35,490 & 5.261 \\
\hline 10 & 84,831 & 9.863 \\
\hline 20 & 254,116 & 20.009 \\
\hline
\end{tabular}

TABLE I -Standard Curve Analysis 


\begin{tabular}{|c|c|c|c|c|c|c|}
\hline $\begin{array}{c}\text { Conc. } \\
\mu \mathrm{mol} / \mathrm{ml}\end{array}$ & Avg FSU & $\begin{array}{c}\text { Avg } \mu \mathrm{mol} / \mathrm{mL} \text { in } \\
\text { rinse }\end{array}$ & \%Grafted & $+/-\mathrm{SD}$ & $\mu \mathrm{g} / \mathrm{cm}^{2}$ & $+/$-SD \\
\hline 0.026 & 169 & 0.036 & -42.94 & 46.76 & $0 *$ & $0 *$ \\
\hline 5.26 & 33,755 & 4.98 & 5.29 & 6.98 & 9.09 & 12.00 \\
\hline 9.86 & 81,089 & 9.37 & 5.04 & 3.90 & 17.33 & 13.42 \\
\hline 20.01 & 246,532 & 19.13 & 4.40 & 6.88 & 30.25 & 47.31 \\
\hline
\end{tabular}

TABLE II - Quantification of PEG-RGDS Coating Fluorescence

*Since percent attachment is less than zero, no PEG-RGDS-Fluor 488 is present.

\begin{tabular}{|c|c|c|}
\hline \multicolumn{2}{|c|}{ T-Test $\mathrm{p}$-values } & $\mu \mathrm{g} / \mathrm{cm}^{2}$ \\
\hline Group & Vs & $\mathrm{p}$-value \\
\hline 0 & 5 & 0.113 \\
\hline 0 & 10 & 0.041 \\
\hline 0 & 20 & 0.145 \\
\hline 5 & 10 & 0.143 \\
\hline 5 & 20 & 0.233 \\
\hline 10 & 20 & 0.274 \\
\hline
\end{tabular}

TABLE III -Average $\mu \mathrm{g} / \mathrm{cm}^{2}$ p-values. Yellow indicates significance.

In generating a standard curve, it was noted that the best trend-line fit was a second order polynomial. Using this generated curve, each individual measurement was first translated into the values shown in Table I. Table II shows the same values calculated as percent grafted and micrograms per centimeter squared. Since the standard curve technique recorded a negative percent attachment for the negative control gels, the concentration was assumed to be zero and no concentration was calculated for this group. Total percent attachment for each group was shown to be between 4 and 5 percent, which is less than the measured yield by Hahn et al. using a Ninhydrin assay (Hahn et al., 2006) for fluorescently tagged PEG-RGDS for PEG hydrogels and near identical preparation processes. The increased surface concentration grafted may also be due to the use of a smaller molecular weight PEG for the hydrogel itself (3400 vs 6000), increasing free acrylates on the surface and increasing RGDS 
grafting. For the mass per unit area of RGDS coverage results, only the 0 and 10 $\mu \mathrm{mol} / \mathrm{mL}$ groups were observed to be significantly different from one another using a paired t-test, most likely due to the inaccuracies in the analysis technique performed. As a result, the gel study was further analyzed using confocal microscopy images.

\section{B. Quantification of PEG-RGDS Coating (Confocal)}

After polymerizing gels using the same protocol as the previous quantification method, each gel was imaged using confocal microscopy to observe the homogeneity of the coating as well as obtain a greyscale intensity value for comparison of each group. This was obtained by the creation of a maximum intensity image composed of $21 \mathrm{Z}$ stacks each one-micron apart. The stack begun immediately before fluorescence was detected and completed when the fluorescent intensity visibly diminished. Representative images of each group are shown in Figure 6. The average values obtained from each group are displayed graphically in Figure 7, while the p-values obtained from a paired t-test is listed in Table IV. 


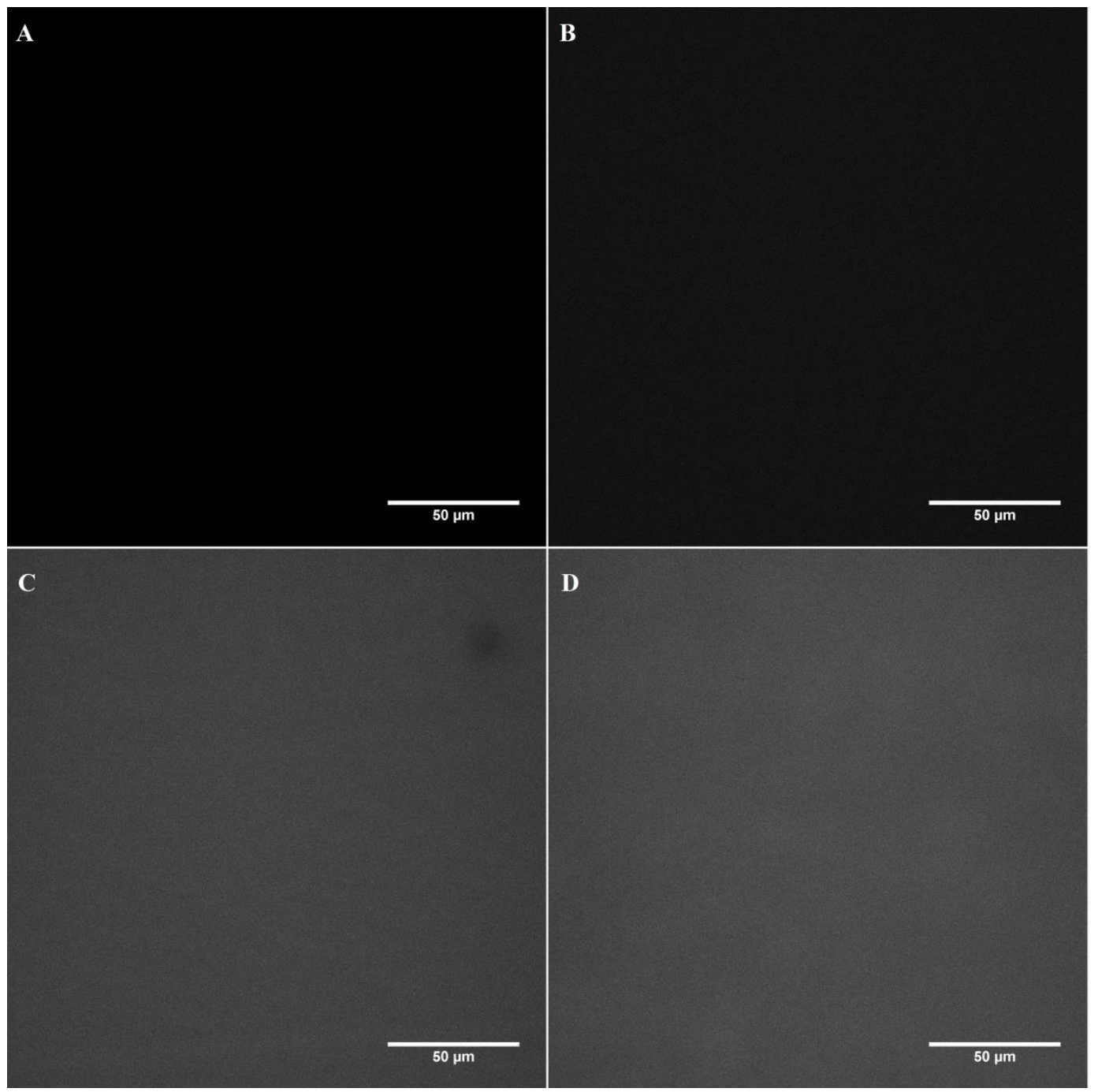

FIGURE 6- PEG-RGDS-Fluor 488 Hydrogels. Sample images of A) 0 B) 5 C) 10 and D) $20 \mu \mathrm{mol} / \mathrm{mL}$ groups. Scale bar represents $50 \mu \mathrm{m}$. 


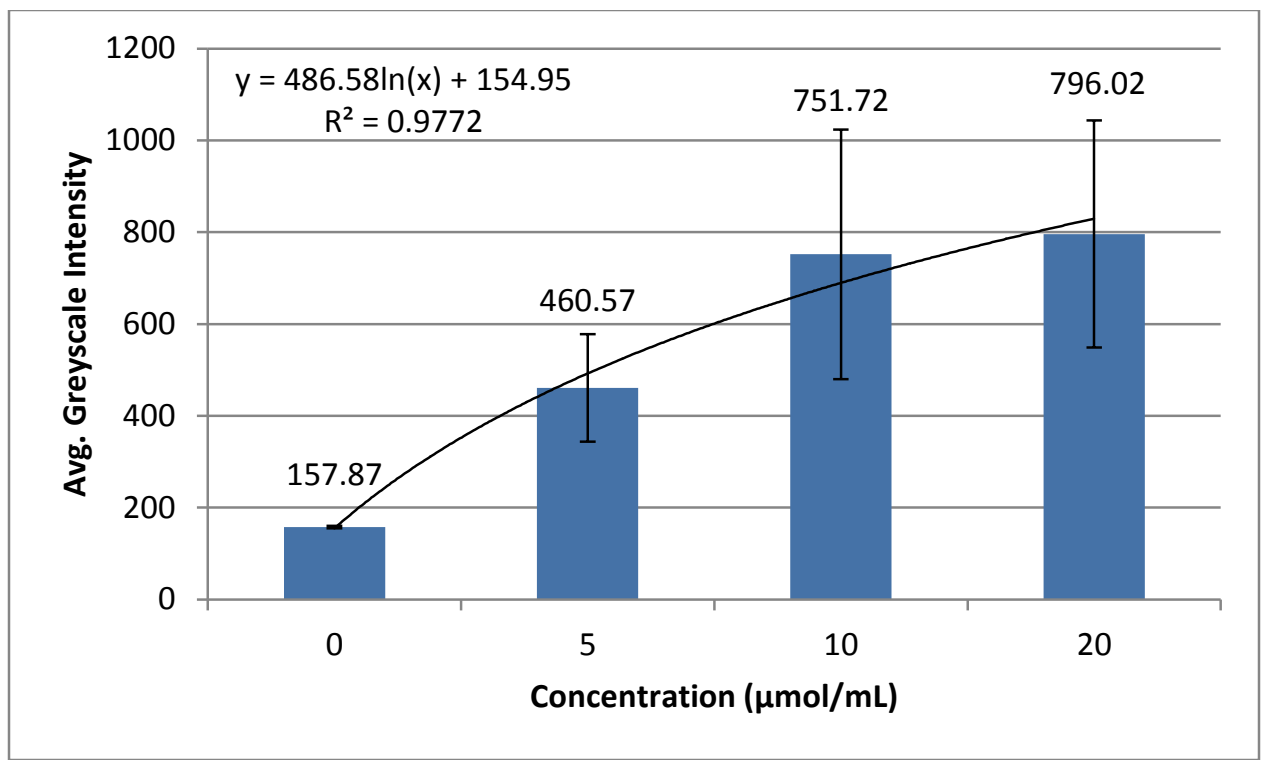

FIGURE 7- Concentration vs. Average Greyscale Intensity

\begin{tabular}{|c|c|c|}
\hline \multicolumn{2}{|c|}{ t-test } & Intensity \\
\hline Group & Vs & p-value \\
\hline 0 & 5 & 0.007 \\
\hline 0 & 10 & 0.011 \\
\hline 0 & 20 & 0.007 \\
\hline 5 & 10 & 0.043 \\
\hline 5 & 20 & 0.048 \\
\hline 10 & 20 & 0.436 \\
\hline
\end{tabular}

TABLE IV -Average Greyscale Intensity p-values. Yellow indicates significance.

The images captured in this study showed highly homogeneous distributions of the RGDS peptide on the PEG hydrogel via fluorescence, clearly demonstrating that the PEG-RGDS-Fluor 488 coating is evenly distributed across the surface. All raw images acquired are shown in Appendix I. The image intensity also decreased as Z-stacks progressed further into each gel, revealing that the majority of the attached PEGRGDS-Fluor 488 is on the surface of the gel.

Overall, the average intensity measured for each gel group showed significantly less coefficient of variance than what was shown using the previous technique. This 
method also allowed for statistically significant differences to be shown between each group except for 10 and $20 \mu \mathrm{mol} / \mathrm{mL}$. The intensity values seemed to plateau near $20 \mu \mathrm{mol} / \mathrm{mL}$, indicating that a maximum concentration may be deposited on the surface within the polymerization time tested. Interestingly, the $\mathrm{R}^{2}$ value for a linear trend-line intersecting at the intensity recorded at the $0 \mu \mathrm{mol} / \mathrm{mL}$ group is 0.9259 , even though the logarithmic trend shown in the figure displays a higher $\mathrm{R}^{2}(0.9772)$. Larger sample sizes analyzed for each group may reduce the coefficient of variation further; thereby, decreasing the coefficient of variation values, improving the linear $\mathrm{R}^{2}$ value and the level of significance for all groups. In general, this technique proved to be a better tool for determining quantification of RGDS attached to the surface, since lower variances were observed between each group as well as providing a visual confirmation that the RGDS was homogeneously grafted on the surface of the gel.

\section{Quantification of Cellular Confluency on Hydrogels}

Cell adhesion was also measured for each sample group in order to determine whether or not these concentrations allowed significantly different percentages of attached cells compared to fibronectin coated coverslips. Sample images of cell adhesion for both ARPE and PRPE cells are shown in Figures 8 and 10 and the complete set of raw images for all cell adhesion samples can be found in Appendix II. The results of the analyses performed on all images are shown in Figures 9 and 11 for ARPE-19 and PRPE cell lines, respectively. P-values for paired t-tests on each cell type are displayed in Tables V and VI. 


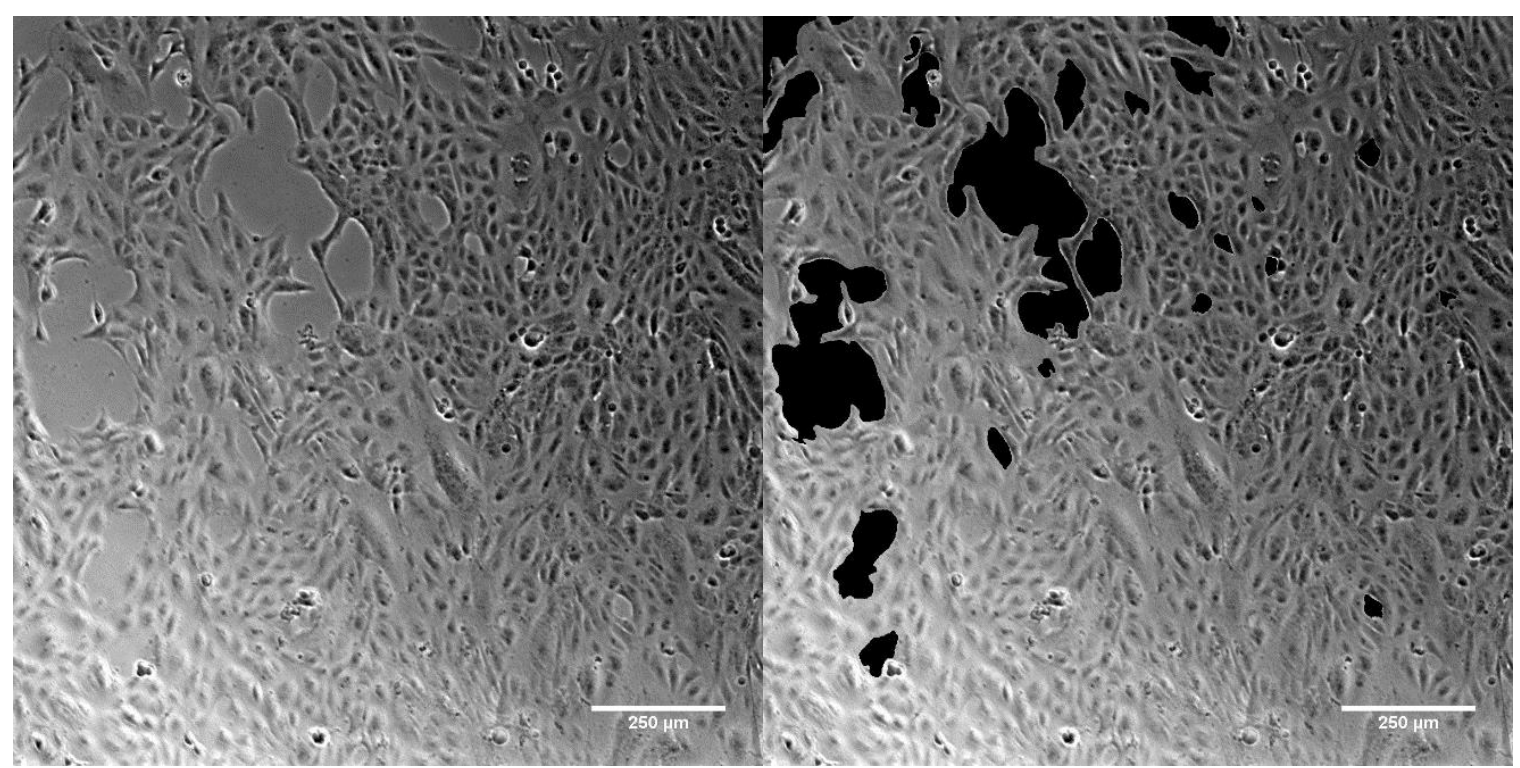

FIGURE 8- ARPE-19 10 $\mu \mathrm{mol} / \mathrm{mL}$ sample. Scale bar represents $250 \mu \mathrm{m}$. Black areas indicate bare areas without cell coverage.

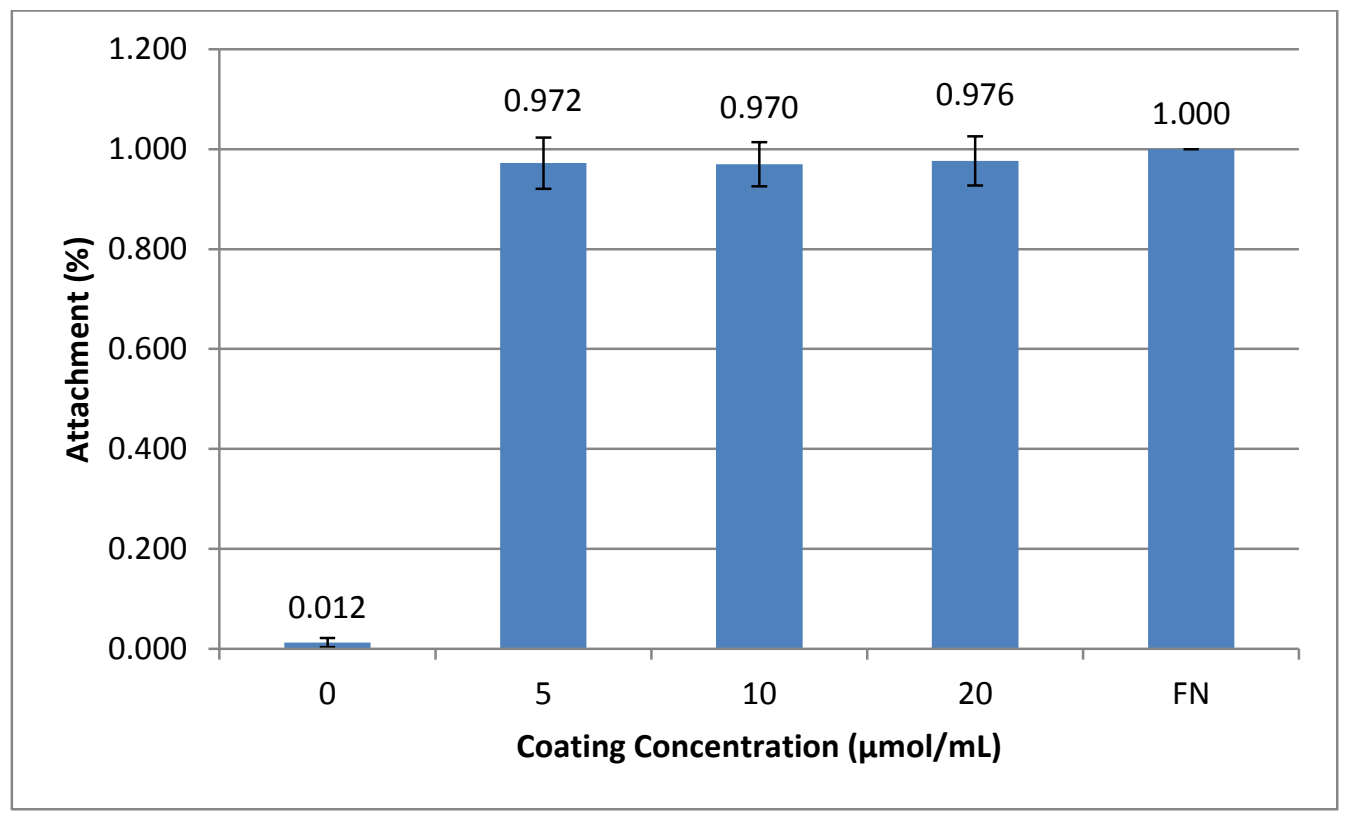

FIGURE 9- ARPE-19 Attachment to Surface. Values represent percent cell attachment. 


\begin{tabular}{|c|c|c|}
\hline t-test & ARPE & confluency \\
\hline Group & vs & p-value \\
\hline 0 & 5 & $<0.0001$ \\
\hline 0 & 10 & $<0.0001$ \\
\hline 0 & 20 & $<0.0001$ \\
\hline 0 & FN & $<0.0001$ \\
\hline 5 & 10 & 0.469 \\
\hline 5 & 20 & 0.437 \\
\hline 5 & FN & 0.071 \\
\hline 10 & 20 & 0.367 \\
\hline 10 & FN & 0.038 \\
\hline 20 & FN & 0.093 \\
\hline
\end{tabular}

TABLE V -ARPE-19 Confluency p-values. Yellow indicates significance.

For the ARPE-19 cells, $\geq 97 \%$ confluency was observed for every gel group, except for the negative controls. A representative image for $10 \mathrm{umol} / \mathrm{ml}$ concentration of RGDS is shown in Figure 11 (all other ARPE-19 confluency images are shown in Appendix II). All RGDS concentration hydrogel groups were found to be significantly different than the non-coated gels ( $0 \mathrm{umol} / \mathrm{ml}$ concentration) as expected. Additionally, the $10 \mu \mathrm{mol} / \mathrm{mL}$ group was found to be significantly different than the fibronectin controls. This may be due to the lack of variance recorded for fibronectin controls since no images were found to have bare areas without cellular growth.

For PRPE cells, $\geq 97 \%$ confluency was observed for every gel group, except for the negative controls. A representative image for the $10 \mathrm{umol} / \mathrm{ml}$ concentration of RGDS is shown in Figure 13 (all other PRPE confluency images are shown in Appendix II). Only the non-coated gels were statistically significant from all other gels as was expected. 


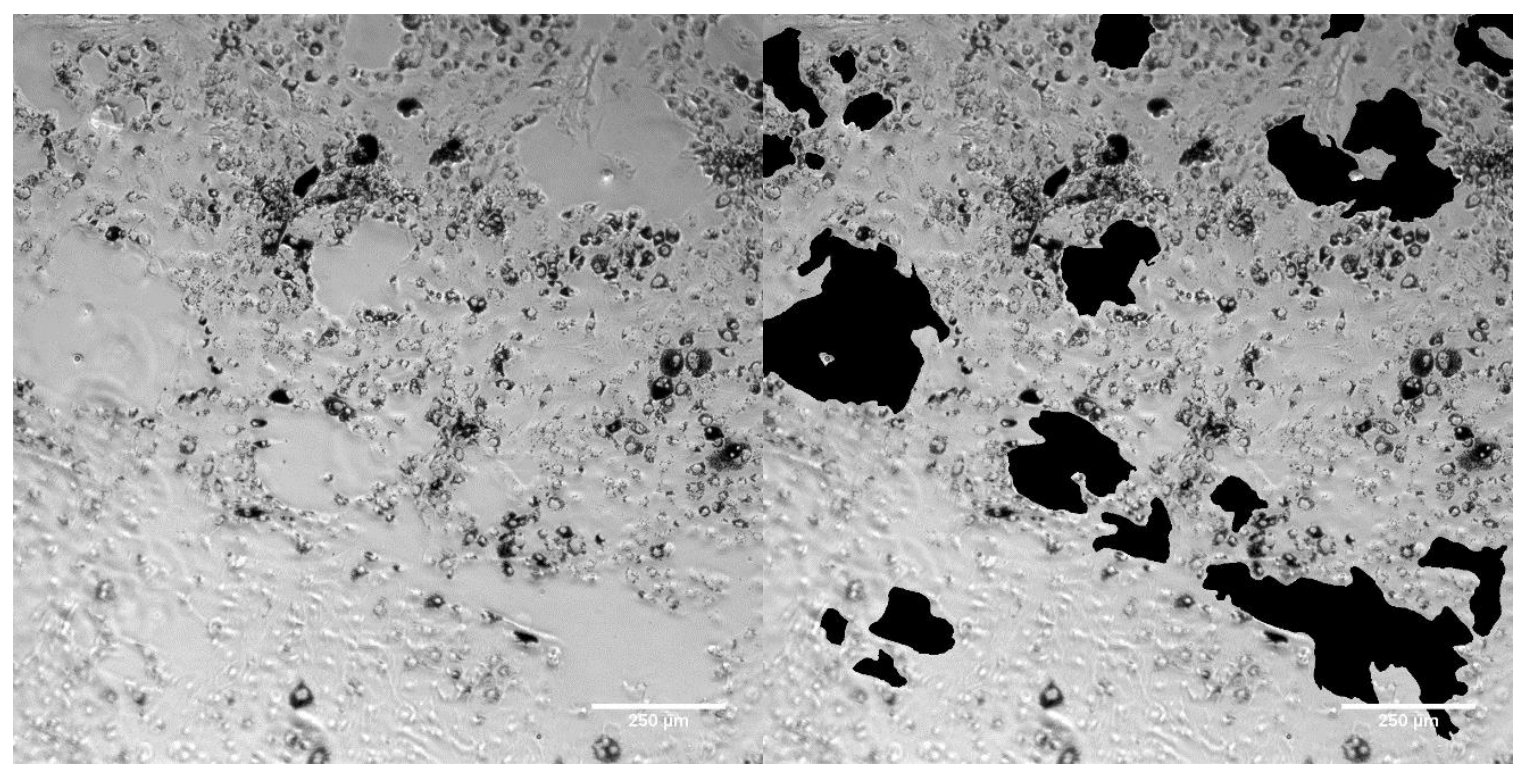

FIGURE 10- PRPE 10 $\mu \mathrm{mol} / \mathrm{mL}$ sample. Scale bar represents $250 \mu \mathrm{m}$. Black areas indicate bare areas without cell coverage.

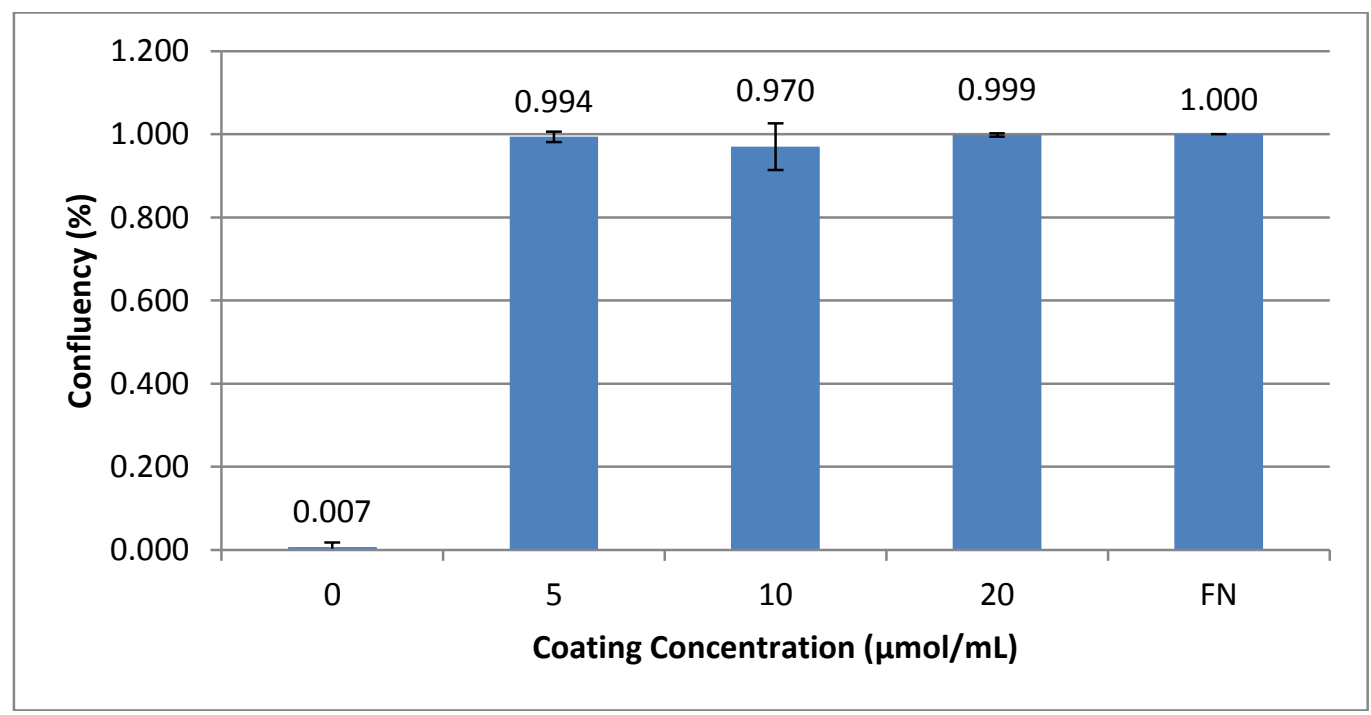

FIGURE 11- PRPE Attachment to Surface. Values represent percent cell attachment. 


\begin{tabular}{|c|c|c|}
\hline t-test & $P$ & confluency \\
\hline Group & vs & $p$-value \\
\hline 0 & 5 & $<0.0001$ \\
\hline 0 & 10 & $<0.0001$ \\
\hline 0 & 20 & $<0.0001$ \\
\hline 0 & FN & $<0.0001$ \\
\hline 5 & 10 & 0.125 \\
\hline 5 & 20 & 0.078 \\
\hline 5 & FN & 0.102 \\
\hline 10 & 20 & 0.087 \\
\hline 10 & FN & 0.075 \\
\hline 20 & FN & 0.173 \\
\hline
\end{tabular}

TABLE VI -PRPE Confluency p-values. Yellow indicates significance.

As for both cell types, nearly all groups measured showed no significant differences when compared to fibronectin controls. This shows that all concentrations are capable of promoting a confluent layer of cells for both cell types. These results also match well with observed PEG-RGDS surface densities $\left(0-100 \mu \mathrm{g} / \mathrm{cm}^{2}\right)$ for human dermal fibroblasts (Moon 2009). Perfecting the gel fabrication procedures to attain smoother gel surfaces may increase the observed cell confluency since a small portion of confluency defects appeared to be due to imperfections in the gel surface. The imperfections may have been a result of bubble formation during the coating procedure since RPE cells tend to prefer a flat surface in order to fully propagate (Scherzer 2010). As for achieving an optimal concentration of PEG-RGDS for RPE cell adhesion and allowing cells to migrate onto the surface, one would need to select a concentration requiring the lowest amount of PEG-RGDS while still supporting appropriate cell morphology and differentiation in order to decrease overall production costs. 


\section{Determination of RPE Differentiation: ZO-1 Cell Circularity}

RPE cellular morphology was also observed using immunocytochemistry techniques. All groups were coated with cells at confluent densities and fixed after 7 days. A sample size of two per group was used for staining each time the experiment was executed due to the large amount of time required to image each sample. All sample images were then analyzed using ImageJ and Minitab 16. A representative image for $10 \mathrm{umol} / \mathrm{ml}$ concentration of RGDS is shown in Figure 12 (all other ZO-1 images are shown in Appendix III). Analyzed gels are also located in Appendix III and are labeled as 'ZO-1 analysis'. The total number of cells measured as well as number of gels analyzed are listed in Table VII. Only one image in the $0 \mu \mathrm{mol} / \mathrm{mL}$ gels was found to have an attached cell layer; however, based on the fact that this layer of cells was not adhered to the substrate, was folded over on itself and did not demonstrate confluency, this image was not included in the analysis.

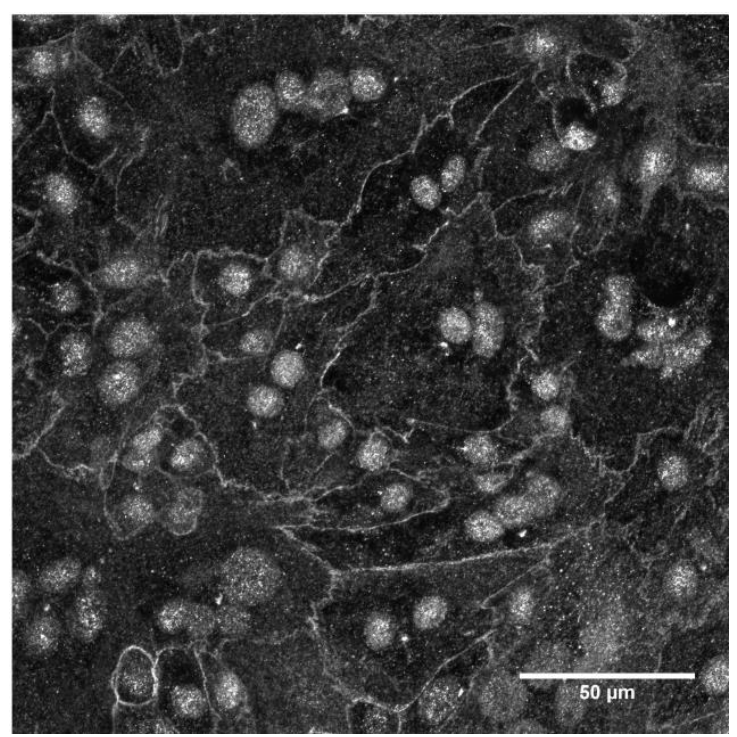

FIGURE 12- PRPE 10-2-1 (5/17/13) 


\begin{tabular}{|c|c|c|}
\hline Sample & Gel \# & Counted \\
\hline ARPE-5 & 1 & 8 \\
\hline ARPE-10 & 1 & 5 \\
\hline & 2 & 12 \\
\hline ARPE-20 & 1 & 5 \\
\hline & 2 & 16 \\
\hline ARPE-FN & 1 & 3 \\
\hline & 2 & 1 \\
\hline & 3 & 3 \\
\hline PRPE-5 & 1 & 13 \\
\hline & 2 & 7 \\
\hline & 3 & 11 \\
\hline PRPE-10 & 1 & 13 \\
\hline & 2 & 11 \\
\hline & 3 & 9 \\
\hline & 4 & 11 \\
\hline PRPE-20 & 1 & 11 \\
\hline & 3 & 5 \\
\hline & 4 & 9 \\
\hline PRPE-FN & 1 & 11 \\
\hline & 2 & 13 \\
\hline & 3 & 15 \\
\hline & 4 & 21 \\
\hline
\end{tabular}

TABLE VII -Gel and Cell Number Count

Cellular circularity is determined using Equation 1. This equation provides a value between zero and one, with one being a perfect circle. As a cell becomes more densely packed and more epithelioid in shape, the circularity value of the cellular boundary approaches one. Therefore, the higher the circularity value the more epithelioid and differentiated the cell.

$$
f_{\text {circ }}=\frac{4 \pi \cdot \text { Area }}{\text { Perimeter }^{2}} \quad \text { Equation } 1
$$


Due to the inability to discern proper ZO-1 expression in some images, only a few cells were analyzed from each gel, especially in the ARPE-19 images. A minimum of four gels were analyzed for all samples, but only images containing ZO-1 expression for analysis are included within the numerical values and figures. Figure 13 also shows an example of the differences between images, where the left image illustrates cells in a more epithelioid shape, which corresponds to higher circularity values. The image on the right demonstrates cells that display more fibroblastic phenotypic behavior, with smaller circularity values and significantly less ZO-1 expression around the perimeter of the cells.
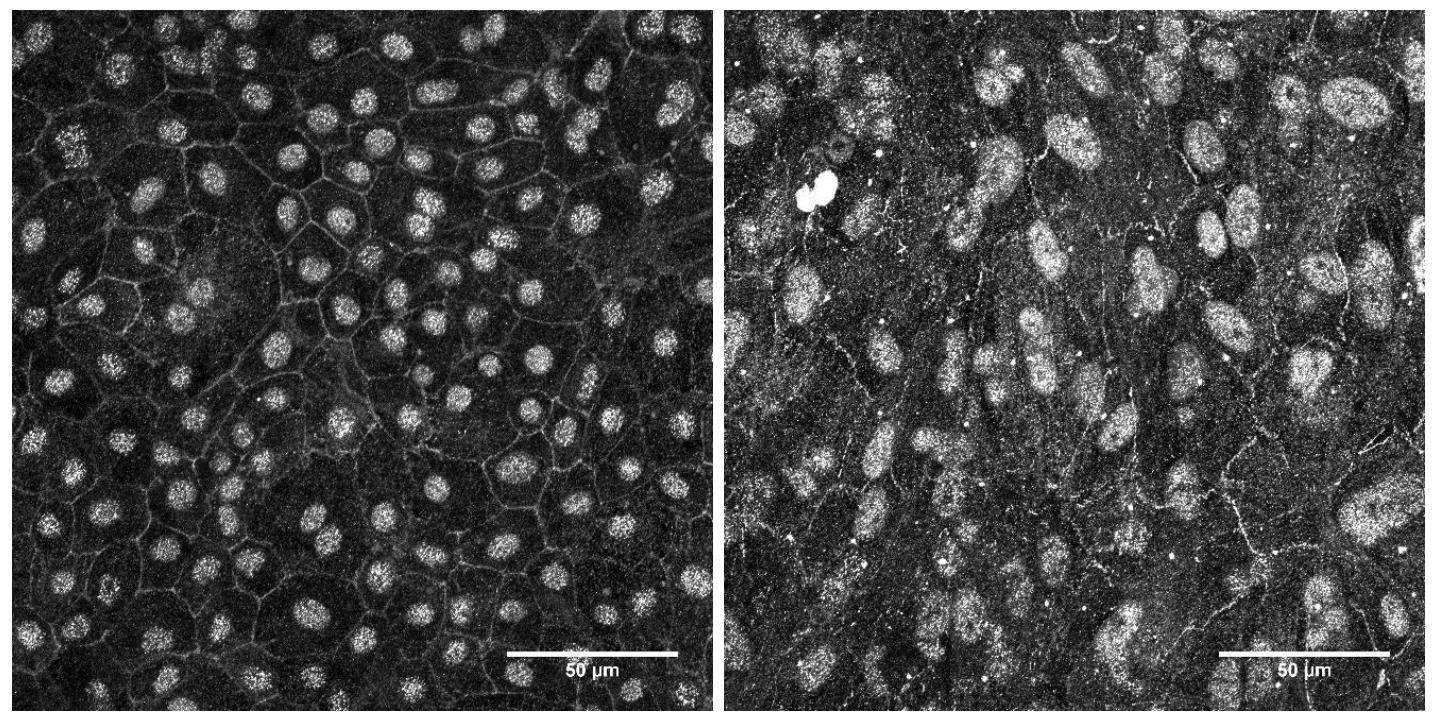

FIGURE 13- Left - PRPE cells on fibronectin, image FN-2-2; and, Right - ARPE cells on fibronectin, image FN1-4. The bright oval/circular spots indicate nuclei and the bright lines surrounding the nuclei identify $\mathrm{ZO}-1$ located on the periphery of the cells.

The following data on cell circularity was obtained from the analysis of the ZO-1 images obtained from each group (Figure 14). Only one RGDS concentration pair was found to be statistically significant for ARPE-19 cells (10 and $20 \mu \mathrm{mol} / \mathrm{mL})$, although the RGDS concentration pair of $10 \mu \mathrm{mol} / \mathrm{mL}$ and FN show a p-value of 0.08 . For the 
PRPEs, all RGDS concentration pairs were found to be statistically significant from the fibronectin controls. This shows that fibronectin promotes a more epithelioid shape than all three RGDS gel concentrations. In comparing differences between cell types at the same RGDS gel concentrations, t-test analysis showed a significant difference for all cases, except for the $10 \mu \mathrm{mol} / \mathrm{mL}$, with PRPE cells displaying a higher circularity (Table VIII). This is potentially due to a smaller sample size within ARPE-19 samples (17), increasing the potential variability of the sample and decreasing its significance compared to the number of PRPE samples (44).

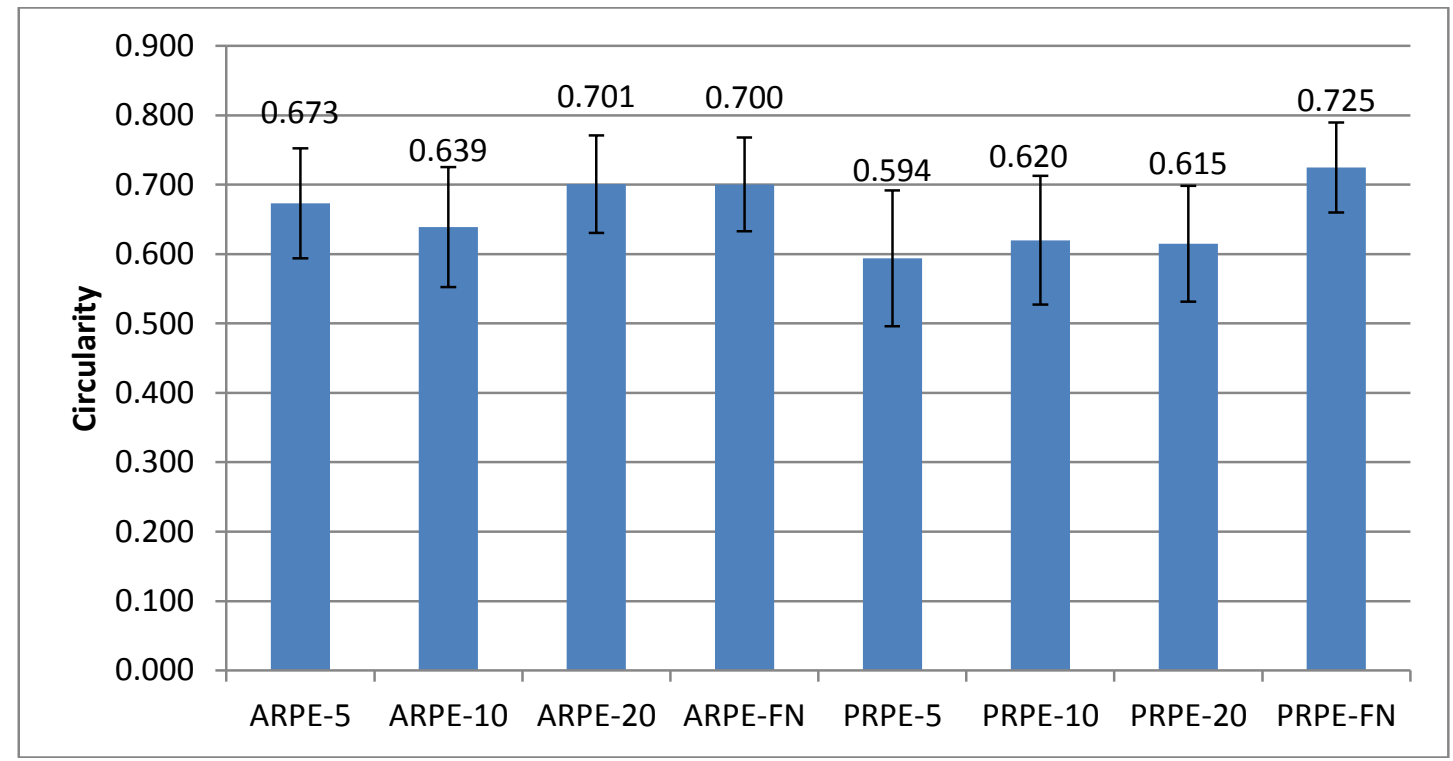

FIGURE 14- Circularity by Group. Bars indicate standard deviation.

\begin{tabular}{|c|c|c|}
\hline t-test & A & Circularity \\
\hline Group & vs & p-value \\
\hline 5 & 10 & 0.176 \\
\hline 5 & 20 & 0.187 \\
\hline 5 & FN & 0.270 \\
\hline 10 & 20 & 0.011 \\
\hline 10 & FN & 0.080 \\
\hline 20 & FN & 0.495 \\
\hline
\end{tabular}

\begin{tabular}{|c|c|c|}
\hline t-test & $\mathrm{P}$ & Circularity \\
\hline Group & vs & $\mathrm{p}$-value \\
\hline 5 & 10 & 0.132 \\
\hline 5 & 20 & 0.311 \\
\hline 5 & $\mathrm{FN}$ & 0.000 \\
\hline 10 & 20 & 0.288 \\
\hline 10 & FN & 0.000 \\
\hline 20 & FN & 0.000 \\
\hline
\end{tabular}

\begin{tabular}{|c|c|c|}
\hline t-test & & Circularity \\
\hline Group & vs & p-value \\
\hline A-5 & P-5 & 0.022 \\
\hline A-10 & P-10 & 0.237 \\
\hline A-20 & P-20 & 0.000 \\
\hline A-FN & P-FN & 0.038 \\
\hline
\end{tabular}

TABLE VIII -Circularity p-values Left- ARPE-19 groups. Center- PRPE groups. Right- ARPE vs. PRPE groups. Yellow indicates significance. 
A multi-factor ANOVA following the General Linear Model was also performed using Minitab 16 to observe significance between concentrations, gels and cell types. Three ANOVAs were performed; one ANOVA for each cell type as well as an ANOVA for all samples. These results are listed in Table IX. Individual PRPE gels are shown to be a significant factor in the ANOVA as well as between the different RGDS gels concentrations. Cell type, concentration and RGDS gel concentration are also shown to be significantly different when comparing the ANOVA data between cell types. A Tukey's Test analysis to determine circularity differences between gels of different RGDS concentrations is shown in Table X, with the PRPE data showing a statistically significant difference between fibronectin and the rest of the RGDS concentrations, matching the p-values in Table VIII.

\begin{tabular}{|c|c|}
\hline ARPE & Circularity \\
\hline Factor & $\mathrm{p}$-value \\
\hline Concentration & 0.069 \\
\hline Gel(Conc) & 0.317 \\
\hline R-Sq(Adj) & 0.0833 \\
\hline
\end{tabular}

\begin{tabular}{|c|c|}
\hline PRPE & Circularity \\
\hline Factor & p-value \\
\hline Concentration & 0.000 \\
\hline Gel(Conc) & 0.007 \\
\hline R-Sq(Adj) & 0.3769 \\
\hline
\end{tabular}

\begin{tabular}{|c|c|}
\hline ARPE/PRPE & Circularity \\
\hline Factor & $\mathrm{p}$-value \\
\hline Type & 0.002 \\
\hline Concentration & 0.000 \\
\hline Gel(Conc) & 0.027 \\
\hline R-Sq(Adj) & 0.2916 \\
\hline
\end{tabular}

TABLE IX -ANOVA for Circularity. Left- ARPE-19 groups. Center- PRPE groups. Right- ARPE vs. PRPE groups. Yellow indicates significance.

\begin{tabular}{|c|c|c|c|}
\hline ARPE & Group & PRPE & Group \\
\hline 5 & $A$ & 5 & $B$ \\
\hline 10 & $A$ & 10 & $B$ \\
\hline 20 & $A$ & 20 & $B$ \\
\hline FN & $A$ & FN & $A$ \\
\hline
\end{tabular}

TABLE X -Tukey's Test for Circularity vs. Concentration. Letters indicate groups significant from one another.

In general, the circularity values recorded for ARPE-19 cells show no significance between groups. PRPE groups for circularity also show significantly smaller values than fibronectin. This shows that RGDS does not significantly promote higher 
circularity values than fibronectin itself. Further evaluation should help indicate if this trend of conveying dedifferentiation is also indicated through the rest of the parameters measured below.

\section{E. Determination of RPE Differentiation: ZO-1 Cell Perimeter}

Analysis of ZO-1 images also included obtaining cell perimeter values. The following data on cell perimeter was obtained from the analysis of ZO-1 images obtained from each group (Figure 15).

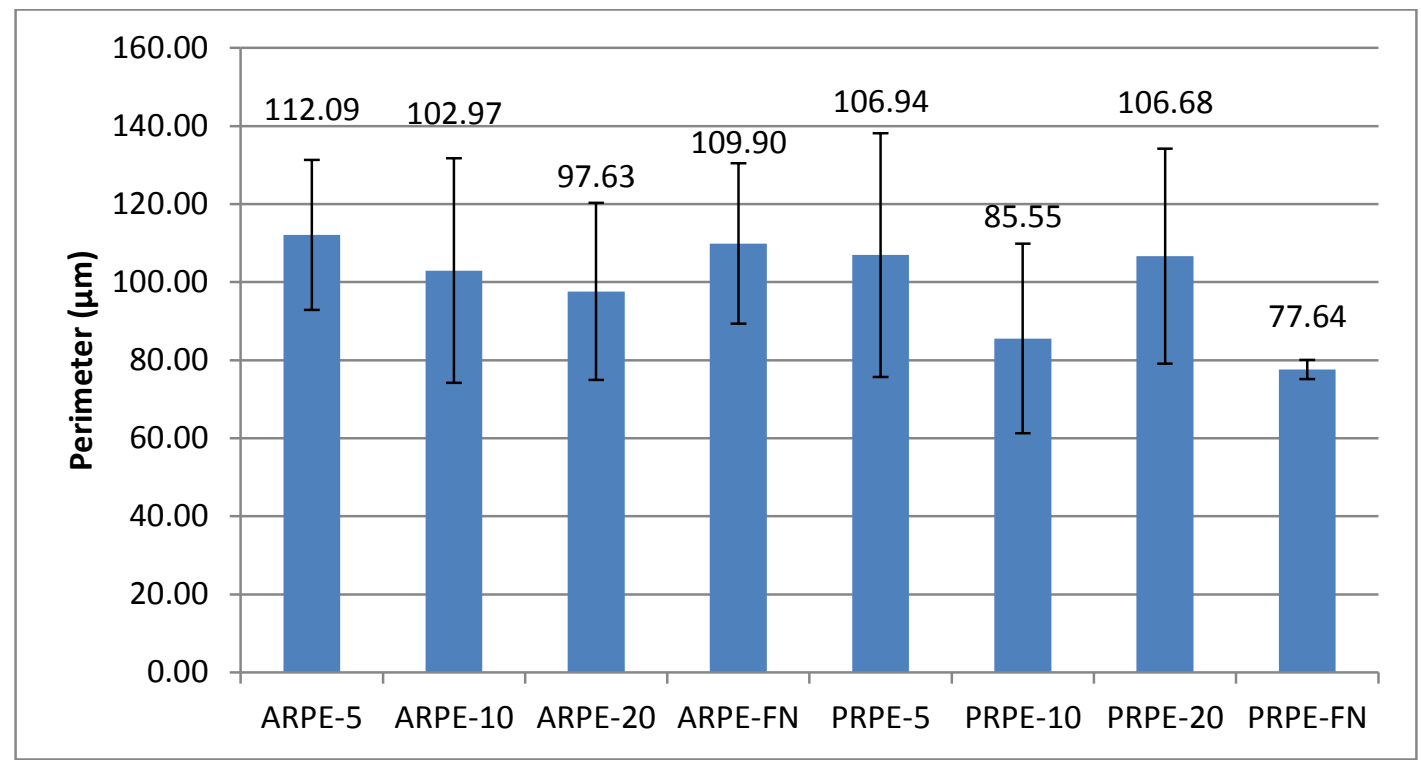

FIGURE 15- Perimeter by Group. Bars indicate standard deviation.

\begin{tabular}{|c|c|c|}
\hline t-test & ARPE & Perim \\
\hline Group & vs & p-value \\
\hline 5 & 10 & 0.214 \\
\hline 5 & 20 & 0.061 \\
\hline 5 & FN & 0.417 \\
\hline 10 & 20 & 0.266 \\
\hline 10 & FN & 0.286 \\
\hline 20 & FN & 0.108 \\
\hline
\end{tabular}

\begin{tabular}{|c|c|c|}
\hline t-test & PRPE & Perim \\
\hline Group & vs & p-value \\
\hline 5 & 10 & 0.001 \\
\hline 5 & 20 & 0.065 \\
\hline 5 & FN & 0.000 \\
\hline 10 & 20 & 0.000 \\
\hline 10 & FN & 0.124 \\
\hline 20 & FN & 0.000 \\
\hline
\end{tabular}

\begin{tabular}{|c|c|c|}
\hline t-test & & Perim \\
\hline Group & vs & p-value \\
\hline A-5 & P-5 & 0.330 \\
\hline A-10 & P-10 & 0.012 \\
\hline A-20 & P-20 & 0.013 \\
\hline A-FN & P-FN & 0.001 \\
\hline
\end{tabular}

TABLE XI -Perimeter p-values Left- ARPE-19 groups. Center- PRPE groups. Right- ARPE vs. PRPE groups. Yellow indicates significance. 
Of the data collected for ARPE-19 cells, no statistically significant difference was found between any of the samples, although the difference between 5 and $20 \mu \mathrm{mol} / \mathrm{mL}$ is less than 0.07 (Table XI). This indicates that with increased statistical samples, the variability between these two groups may become significant. This observation may also be an indication that since ARPE-19s are an immortalized cell line, they may respond significantly different to substrate integrin concentrations. Although no statistically significant differences were found between ARPE-19 groups, the same cannot be said for PRPEs seeded on gels with different RGDS concentrations. All RGDS gel concentrations but $10 \mathrm{~mol} / \mathrm{mL}$ show significant differences from the fibronectin controls as well as a significant difference between 10 and $20 \mu \mathrm{mol} / \mathrm{mL}$ and between 5 and $10 \mu \mathrm{mol} / \mathrm{mL}$. Interestingly, no significant difference is noted between these two paired groups for PRPE when measured for circularity. This may indicate that an increase in perimeter does not necessarily coincide or directly correlate with a fibroblastic morphology. This is shown in the reorganization of RPEs in vivo when cells begin to die with age. RPEs will tend to spread circumferentially to fill in small defects instead of becoming polarized (Grierson et al. 1994).

A multi-factor ANOVA following the General Linear Model was also performed using Minitab 16 to observe significant differences between concentrations, gels and cell types. Three ANOVAs were performed; one ANOVA for each cell type as well as an ANOVA for all samples. These results are listed in Tables XII. Tukey's Test for perimeter is also shown in Table XIII. 


\begin{tabular}{|c|c|c|c|c|c|}
\hline ARPE & Perimeter & PRPE & Perimeter & ARPE/PRPE & Perimeter \\
\hline Factor & $p$-value & Factor & $p$-value & Factor & $p$-value \\
\hline Concentration & 0.269 & Concentration & 0.000 & Type & 0.005 \\
\hline Gel(Conc) & 0.090 & Gel(Conc) & 0.099 & Concentration & 0.000 \\
\hline R-Sq(Adj) & 0.0837 & $\mathrm{R}-\mathrm{Sq}(\mathrm{Adj})$ & 0.2111 & Gel(Conc) & 0.163 \\
\hline & & & & R-Sq(Adj) & 0.1596 \\
\hline
\end{tabular}

TABLE XII -ANOVA for Perimeter. Left- ARPE-19 groups. Center- PRPE groups. Right- ARPE vs. PRPE groups. Yellow indicates significance.

\begin{tabular}{|c|c|c|c|}
\hline ARPE & Group & PRPE & Group \\
\hline 5 & $A$ & 5 & A \\
\hline 10 & A & 10 & B \\
\hline 20 & $A$ & 20 & A \\
\hline $\mathrm{FN}$ & A & FN & $B$ \\
\hline
\end{tabular}

TABLE XIII -Tukey's Test for Perimeter vs. Concentration. Letters indicate groups significant from one another.

The ANOVA in Table XII also shows similar results as the t-tests above. PRPE concentration is shown to be significant factor in the ANOVA. Type and concentration are also shown to be significant with the ANOVA data including both cell types. Tukey's Test with PRPEs shows that there are two significantly different groups, 5 and $20 \mu \mathrm{mol} / \mathrm{mL}$ as compared to $10 \mu \mathrm{mol} / \mathrm{mL}$ and fibronectin, matching the significance shown between groups in Table XI.

In general, perimeter values recorded for ARPE-19 cells also show minimal variation between groups with no specific trend with increasing RGDS concentration. PRPE groups also show similar data. This indicates that RGDS does not significantly promote a differentiated state related to a smaller perimeter. Again, further evaluation of the last two parameters should help convey if this trend of parameters demonstrating a dedifferentiated, fibroblastic morphology. 


\section{F. Determination of RPE Differentiation: ZO-1 Cell Area}

ZO-1 images were also measured for cellular area. The following data on cell area was obtained from the analysis of ZO-1 images for each group (Figure 16).

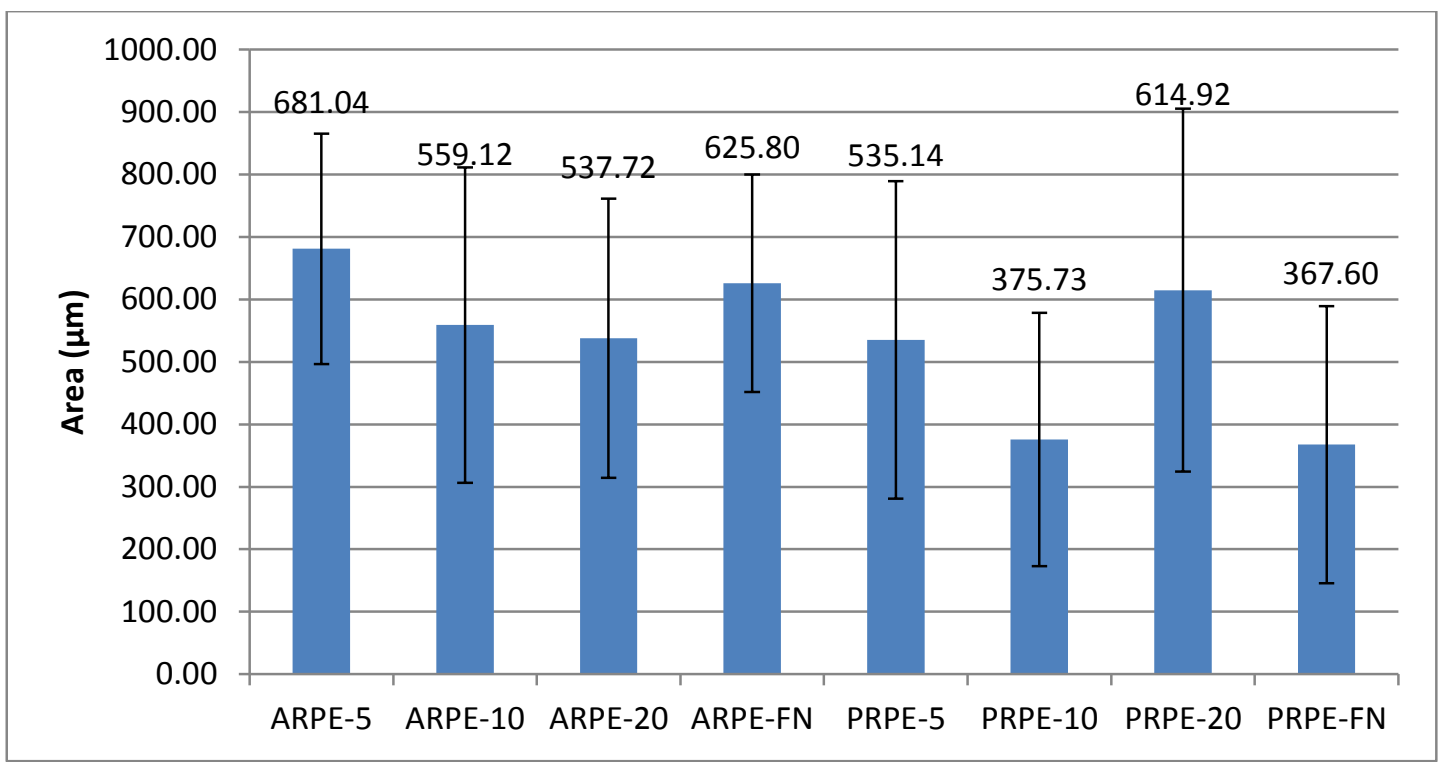

FIGURE 16- Area by Group. Bars indicate standard deviation.

\begin{tabular}{|c|c|c|}
\hline t-test & ARPE & Area \\
\hline Group & vs & p-value \\
\hline 5 & 10 & 0.120 \\
\hline 5 & 20 & 0.059 \\
\hline 5 & FN & 0.282 \\
\hline 10 & 20 & 0.393 \\
\hline 10 & FN & 0.267 \\
\hline 20 & FN & 0.176 \\
\hline
\end{tabular}

\begin{tabular}{|c|c|c|}
\hline t-test & PRPE & Area \\
\hline Group & vs & p-value \\
\hline 5 & 10 & 0.002 \\
\hline 5 & 20 & 0.017 \\
\hline 5 & FN & 0.005 \\
\hline 10 & 20 & 0.000 \\
\hline 10 & FN & 0.432 \\
\hline 20 & FN & 0.000 \\
\hline
\end{tabular}

\begin{tabular}{|c|c|c|}
\hline t-test & & Area \\
\hline Group & vs & p-value \\
\hline A-5 & P-5 & 0.070 \\
\hline A-10 & P-10 & 0.003 \\
\hline A-20 & P-20 & 0.030 \\
\hline A-FN & P-FN & 0.001 \\
\hline
\end{tabular}

TABLE XIV -Area p-values. Left- ARPE-19 groups. Center- PRPE groups. Right- ARPE vs. PRPE groups. Yellow indicates significance.

No statistical significance was observed for cell area in ARPE-19 cells, although groups 5 and $20 \mu \mathrm{mol} / \mathrm{mL}$ was shown to be less than 0.06 (Table XIV). Increased cell sample measurements may potentially reduce this $\mathrm{p}$-value, indicating significance 
between these groups. For PRPE cells, 5 and $20 \mu \mathrm{mol} / \mathrm{mL}$ were found to be statistically different from fibronectin controls, whereas $10 \mathrm{umol} / \mathrm{ml}$ was not. Differences between each of the RGDS gel concentrations were also shown to be statistically significantly different. These findings further indicate that increasing RGDS concentrations does not significantly impact morphology. An increase in area is also typically associated with, in vivo, death and rearrangement of the RPEs (Grierson et al., 1994). Since these cells do not proliferate for small defects, their area increases to compensate for the reduction in cell density, which may correlate to the higher area values recorded for both cell types.

A multi-factor ANOVA following the General Linear Model was also performed using Minitab 16 to observe significance between concentrations, gels and cell types. Three ANOVAs were performed; one ANOVA for each cell type as well as an ANOVA for all samples. These results are listed in Tables XV. Tukey's Test for area is also shown in Table XVI.

\begin{tabular}{|c|c|}
\hline ARPE & Area \\
\hline Factor & $p$-value \\
\hline Concentration & 0.368 \\
\hline Gel(Conc) & 0.108 \\
\hline R-Sq(Adj) & 0.0755 \\
\hline
\end{tabular}

\begin{tabular}{|c|c|}
\hline PRPE & Area \\
\hline Factor & $p$-value \\
\hline Concentration & 0.000 \\
\hline Gel(Conc) & 0.053 \\
\hline R-Sq(Adj) & 0.1793 \\
\hline
\end{tabular}

\begin{tabular}{|c|c|}
\hline ARPE/PRPE & Area \\
\hline Factor & $p$-value \\
\hline Type & 0.000 \\
\hline Concentration & 0.003 \\
\hline Gel(Conc) & 0.098 \\
\hline R-Sq(Adj) & 0.1473 \\
\hline
\end{tabular}

TABLE XV -ANOVA for Area. Left- ARPE-19 groups. Center- PRPE groups. Right- ARPE vs. PRPE groups. Yellow indicates significance.

\begin{tabular}{|c|c|}
\hline ARPE & Group \\
\hline 5 & A \\
\hline 10 & A \\
\hline 20 & A \\
\hline FN & A \\
\hline
\end{tabular}

\begin{tabular}{|c|c|}
\hline PRPE & Group \\
\hline 5 & A \\
\hline 10 & B \\
\hline 20 & A \\
\hline FN & B \\
\hline
\end{tabular}

TABLE XVI -Tukey's Test for Area vs. Concentration. Letters indicate groups significant from one another. 
The ANOVA in Table XV again shows comparable data as the t-tests above. PRPE concentration is shown to be a significant factor in the ANOVA, revealing statistically significant values recorded for RGDS concentration. Cell type and cell concentration were also significantly different for the ANOVA. Tukey's Test with PRPEs shows that there are two significantly different groups, 5 and $20 \mu \mathrm{mol} / \mathrm{mL}$ as compared to $10 \mu \mathrm{mol} / \mathrm{mL}$ and fibronectin, similar to the significance shown between groups in Table XIV, although the t-tests indicate that 5 and $20 \mu \mathrm{mol} / \mathrm{mL}$ are significantly different. This is due to differences in calculation of significance, but also shows that other factors may decrease the significance between groups, such as recorded individual gel variation.

In general, area values recorded for ARPE-19 cells also show minimal variation between groups with no specific trend. PRPE groups show significance from other concentrations and controls, but no clear trend due to increasing concentration, revealing there may be other potential influences. This indicates that RGDS does not directly promote a differentiated state related to a smaller area, although further evaluation of the last parameter should help convey if the values previously shown truly display a dedifferentiated, fibroblastic morphology.

\section{G. Determination of RPE Differentiation: ZO-1 Aspect Ratio}

Finally, ZO-1 images were also measured for cellular aspect ratio. The following data on cell aspect ratio was obtained from the analysis of ZO-1 images obtained from each group (Figure 17). 


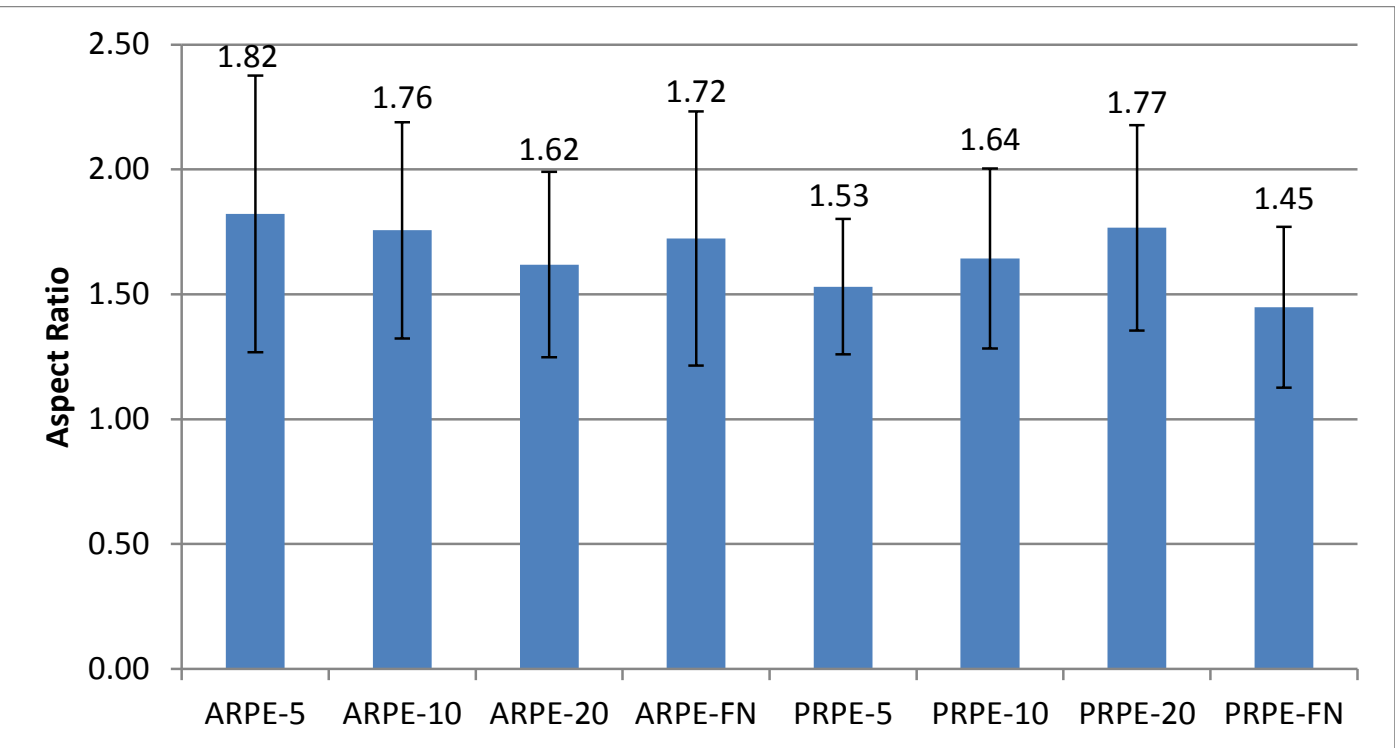

FIGURE 17- Aspect Ratio by Group Bars indicate standard deviation.

\begin{tabular}{|c|l|r|}
\hline t-test & ARPE & Aspect \\
\hline Group & vs & p-value \\
\hline 5 & 10 & 0.377 \\
\hline 5 & 20 & 0.133 \\
\hline 5 & FN & 0.362 \\
\hline 10 & 20 & 0.160 \\
\hline 10 & FN & 0.438 \\
\hline 20 & FN & 0.283 \\
\hline
\end{tabular}

\begin{tabular}{|c|c|r|}
\hline t-test & PRPE & Aspect \\
\hline Group & vs & p-value \\
\hline 5 & 10 & 0.078 \\
\hline 5 & 20 & 0.003 \\
\hline 5 & FN & 0.365 \\
\hline 10 & 20 & 0.022 \\
\hline 10 & FN & 0.033 \\
\hline 20 & FN & 0.000 \\
\hline
\end{tabular}

\begin{tabular}{|l|l|r|}
\hline t-test & & Aspect \\
\hline Group & vs & p-value \\
\hline A-5 & P-5 & 0.021 \\
\hline A-10 & P-10 & 0.163 \\
\hline A-20 & P-20 & 0.045 \\
\hline A-FN & P-FN & 0.022 \\
\hline
\end{tabular}

TABLE XVII-Aspect Ratio p-values. Left- ARPE-19 groups. Center- PRPE groups. Right- ARPE vs. PRPE groups. Yellow indicates significance.

Again, no significant difference was found between any groups in ARPE-19 cells (Table VII). For PRPE, fibronectin controls were significantly different than the 10 and $20 \mu \mathrm{mol} / \mathrm{mL}$ RGDS concentration groups, but not the $5 \mu \mathrm{mol} / \mathrm{mL}$ group. This shows that fibronectin controls promote the smallest aspect ratio, demonstrating a higher cellular surface density and potentially more differentiated state. The $20 \mu \mathrm{mol} / \mathrm{mL}$ group was also found to be significantly different than all other groups. This further indicates that other factors may be effecting morphology, since no proportional relationship is shown by increasing concentrations of RGDS. 
Another interesting finding is the relationship of the $5 \mu \mathrm{mol} / \mathrm{mL}$ PRPE circularity and aspect ratio. This group has the second lowest average circularity value and has the second lowest aspect ratio. For a perfect circle or shapes nearing a circular shape, a high circularity value as well as a low aspect ratio are recorded. This must mean that for this group cells are forming shapes other than the typical epithelioid-hexagon, such as a squared or triangular form. In contrast, PRPE fibronectin controls show an inverse relationship between aspect ratio (low) to circularity (high). This reveals the fibronectin controls are potentially promoting a more differentiated state.

A multi-factor ANOVA following the General Linear Model was also performed using Minitab 16 to observe significance between concentrations, gels and cell types. Three ANOVAs were performed; one ANOVA for each cell type as well as an ANOVA for all samples. These results are listed in Tables XVIII. Tukey's Test for aspect ratio is also shown in Table XIX.

\begin{tabular}{|c|c|}
\hline ARPE & $\begin{array}{c}\text { Aspect } \\
\text { Ratio }\end{array}$ \\
\hline Factor & p-value \\
\hline Concentration & 0.526 \\
\hline Gel(Conc) & 0.117 \\
\hline R-Sq(Adj) & 0.0397 \\
\hline
\end{tabular}

\begin{tabular}{|c|c|}
\hline PRPE & $\begin{array}{c}\text { Aspect } \\
\text { Ratio }\end{array}$ \\
\hline Factor & p-value \\
\hline Concentration & 0.000 \\
\hline Gel(Conc) & 0.042 \\
\hline R-Sq(Adj) & 0.1425 \\
\hline
\end{tabular}

\begin{tabular}{|c|c|}
\hline ARPE/PRPE & $\begin{array}{c}\text { Aspect } \\
\text { Ratio }\end{array}$ \\
\hline Factor & $\mathrm{p}$-value \\
\hline Type & 0.024 \\
\hline Concentration & 0.005 \\
\hline Gel(Conc) & 0.004 \\
\hline R-Sq(Adj) & 0.1283 \\
\hline
\end{tabular}

Table XVIII -ANOVA for Aspect Ratio. Left- ARPE-19 groups. Center- PRPE groups. Right- ARPE vs. PRPE groups. Yellow indicates significance.

\begin{tabular}{|c|c|c|c|}
\hline ARPE & Group & PRPE & Group \\
\hline 5 & $A$ & 5 & $B C$ \\
\hline 10 & A & 10 & $A B$ \\
\hline 20 & A & 20 & A \\
\hline FN & $A$ & FN & $C$ \\
\hline
\end{tabular}

Table XIX Tukey's Test for Aspect Ratio vs. Concentration. Letters indicate groups significant from one another. 
The ANOVA in Table XVIII also shows similar results as the t-tests above. PRPE concentration is shown to be a significant factor in the ANOVA as well as the gel concentrations. Cell type, concentration and gel concentrations were also shown to be significantly different. The Tukey's Test for the PRPEs shows that there are three significantly different groups, 10 and $20 \mu \mathrm{mol} / \mathrm{mL}, 5$ and $10 \mu \mathrm{mol} / \mathrm{mL}$ and $5 \mu \mathrm{mol} / \mathrm{mL}$ and fibronectin, , similar to the significance shown between groups in Table XVII. Although the data shows three significantly different groups, no correlation to increasing RGDS concentration is shown, indicating that these significances may be caused by other factors.

Although ZO-1 measurements provide a quantifiable method for determining cellular differentiation and morphology based on visual parameters, the values obtained for both cell types indicate that the concentrations used for this study were insufficient in promoting epithelioid differentiation similar to fibronectin controls. This may potentially suggest that RGDS may be insufficient in itself to promote the desired morphology of these cells, requiring a combination of other peptides along with RGDS or the use of another adhesion ligand based peptide alone. Future research could potentially include the use of other fibronectin-based peptide sequences shown to enhance the use of RGDS, such as PHSRN (Feng et al., 2004). Other adhesive ligand based peptides may also be of interest, such as sequences based on collagen IV. 


\section{H. Determination of RPE Differentiation: Actin Filament Length and Orientation}

Actin filament distribution was also observed using immunocytochemistry techniques. Samples were stained for rhodamine-phalloidin along with ZO-1 staining as mentioned above. Samples were then analyzed using confocal microscopy and imageJ software to observe the morphological characteristics of F-actin. This experiment was then repeated in order to obtain a sample size large enough to statistically analyze using Minitab 16. All Actin filament images are shown in Appendix III.

In typical RPE morphology, actin filaments are strongly associated with tight junction formation around the perimeter of the cells. As shown in Figure 22, typical actin expression is predominantly found in the same area as ZO-1 and other tight junction related proteins. As RPE cells become more dedifferentiated, the cells become more fibroblastic and express actin stress fibers as well as $\alpha$-smooth muscle actin (Grierson et al., 1994).

When analyzing actin for each group, large amounts of actin were found throughout the volume of the cell. This strongly indicates that stress-related fibers have been produced either due to substrate, soluble or spatially initiated cues. This also indicates the promotion of dedifferentiation into fibroblastic morphologies. These observations also correlate with the aberrant circularity and aspect ratio values obtained through ZO-1 measurements. The promotion of large amounts of intracellular actin also made the ability to distinguish circumferential actin extremely difficult, preventing accurate measurement of circumferential actin length and angle orientation to correlate 
with ZO-1 circularity and aspect ratio values. Sample images are shown in Figure 18 with the left image being PRPE cells on fibronectin. On the top 1/3 of the image, appropriate actin morphology is shown, but only for a few cells. In the same image towards the lower half, an increase in actin is expressed, making cellular boundaries indistinguishable. The right image shows ARPE-19 cells on fibronectin; however, the cell boundaries are indistinguishable in the entire image, making actin length and orientation measurements based on individual cell shape unobtainable.

Figure 19 demonstrates an appropriate actin morphology when RPE cells are adequately differentiated (Lee 2007). Using this morphology, actin length and orientation can be determined and shown to correlate with ZO-1 circularity and aspect ratio values. Actin lengths should be roughly equal on all sides and have a near horizontal distribution of angles between 0 and 180 degrees when cells are adequately differentiated. Any increase in the average length or change in variation from a normal angle distribution would indicate a shift toward a fibroblastic morphology due to the increasing polarity of the cells. A significant increase in intracellular actin expression would suggest a shift to a dedifferentiated state for the cell, as illustrated in the images obtained in this study. 

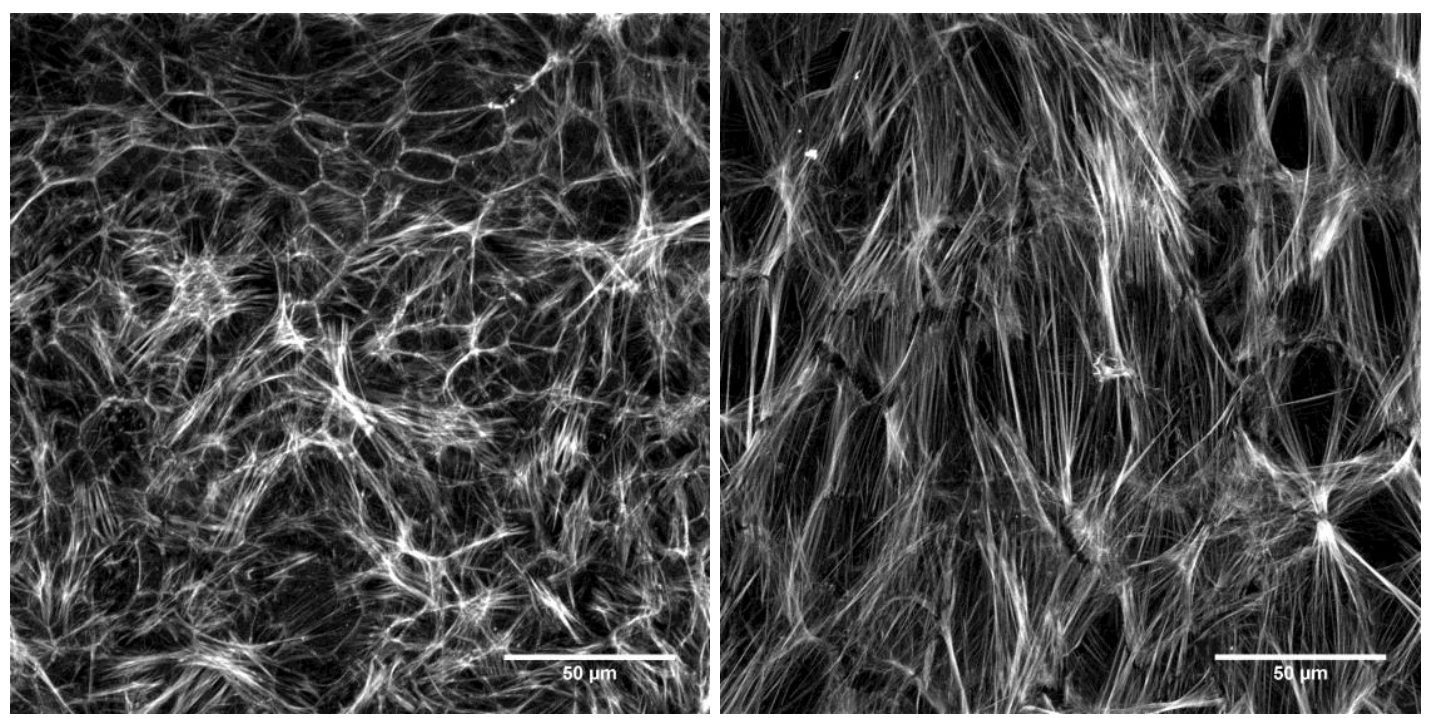

FIGURE 18- Actin Expression on Fibronectin controls. Left- PRPE FN 2-2 Right- ARPE FN 1-4

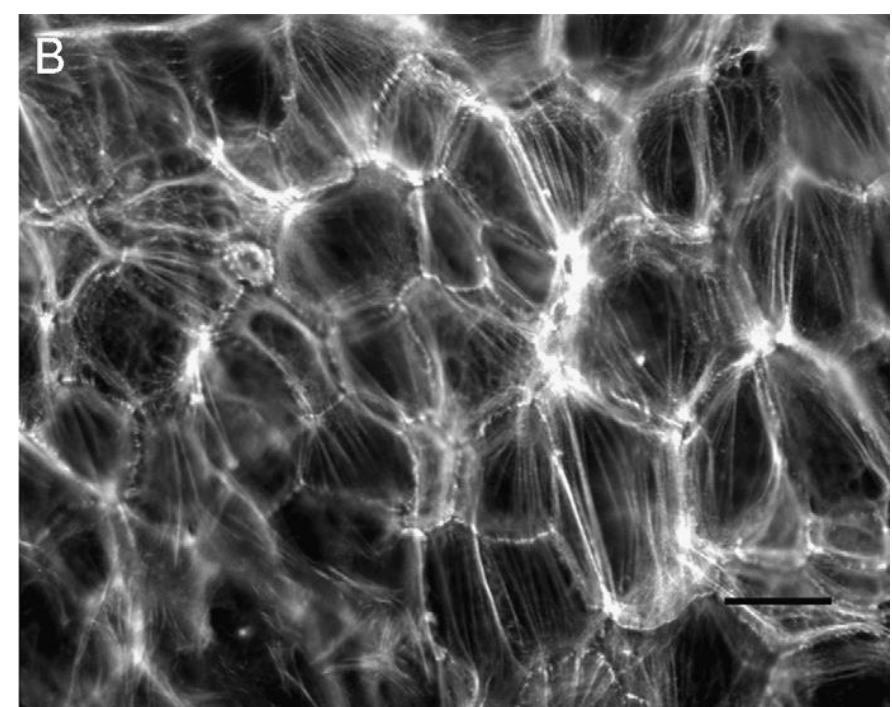

FIGURE 19- Actin Expression in Differentiated RPEs (acquired from Lee 2007) 


\section{CONCLUSIONS}

Four specific aims were analyzed for the duration of this research. First, the technique for surface modification of PEG hydrogels with PEG-RGDS adhesion ligand was quantified using AlexaFluor 488 conjugated PEG-RGDS. This study found that between four and five percent of the total PEG-RGDS solution was polymerized on the surface for each gel group. This showed the functionality of the technique as well as providing a range of adhesion ligand concentration to correlate with cell confluency values for a later specific aim. Further analysis of the fluorescently coated gels using confocal microscopy also proved statistical significance between all coating concentrations except for 10 and $20 \mu \mathrm{mol} / \mathrm{mL}$. It was also shown that the peptide layer is homogeneous throughout the surface of each gel.

For the second specific aim, cellular confluency was compared on differing concentrations of adhesion ligands on PEG hydrogels and compared to non-coated PEG gels and fibronectin coated coverslips. Of the two cell types, only one group was found to be statistically significant from the fibronectin control; the $10 \mu \mathrm{mol} / \mathrm{mL}$ ARPE-19 group. This statistical significance could be caused by the lack of bare gel observed in the fibronectin controls, but should still be considered when trying to optimize the concentration of adhesion ligand for this cell type. All groups of both cell types were also found to be statistically significant from the negative control gels. This shows that in terms of cellular adhesion, the entire range of concentrations could potentially be used for both cell types. 
In terms of differentiated RPE morphology, experimental data showed different results compared to confluency in terms of appropriate concentration of adhesion peptide. In general for ZO-1 studies, low sample sizes in $5 \mu \mathrm{mol} / \mathrm{mL}$ and fibronectin controls in the ARPE-19 studies only seemed to affect results by reducing the variation seen between gels, preventing a visible significant difference between groups. All four parameters also showed statistical significance between cell types, indicating that both of these cell types do not respond identically to one another.

Images taken for R-P were unable to be appropriately analyzed for circumferential actin length and angle compared to ZO-1 due to significant amounts of intracellular actin expression typically only expressed by fibroblastic type RPEs. This shows that both cell types in all groups were expressing some indicators for fibroblastic dedifferentiation.

In conclusion, although the studied levels of PEG-RGDS allowed for near confluent levels of RPE cells on PEG hydrogels, cellular circularity, area, perimeter and aspect ratio did not seem to be related in any proportion to RGDS surface concentration. Fibronectin controls in all cases indicated the closest epithelial morphology based on ZO-1 expression. Other conditions may be potential factors that allow RPEs to become dedifferentiated, such as spatial distribution, material stiffness or lack of certain factors that promote differentiation. In conclusion, the hypothesis that increasing concentrations of PEG-RGDS on PEG-DA hydrogels will significantly promote morphological expression tied to RPE-specific differentiation in both ARPE19 and primary PRPE cell lines was found to be false based on the results of this research. 


\section{RECOMMENDATIONS}

Additional work will be needed in order to fully characterize the state of differentiation for RPE cells seeded on PEG-RGDS coated hydrogels. Factors such as RPE 65 and cellular retinaldehyde binding protein can be stained using similar immunocytochemistry techniques to obtain differentiation information, since these factors are only produced when these cells are in a differentiated state.

In general, more research must be conducted in the promotion and sustainment of RPE cells of differentiated epithelial states after achieving confluency. Fibronectin and fibronectin-based layers have shown to promote excellent migratory and proliferative responses but do not seem to promote differentiation after achieving confluency based on the experimental results discussed above. Alteration of post-confluent media with compounds that promote differentiation may also prove to be beneficial if the end goal is to transplant a fully functioning sheet of cells on the hydrogel into the damaged area. As for using the currently developed material in the wound site to encourage migration and proliferation by existing RPEs, other factors may be required in order to promote a differentiated state after confluency is reached. These factors could be either substrate based, such as collagen or heparin sulfate or based on growth factors that are released after reaching confluency. Other peptides based on other functional domains of integrins could also be included to promote a differentiated state. Other peptide sequences from non-fibronectin based extracellular matrix proteins could also be used such as sequences from collagen IV. Animal studies using PEG-RGDS coated 
hydrogels should also be performed to evaluate the efficiency of proliferation and differentiation in vivo, since potential differentiation factors may be present in the body after confluency is reached and a semi-functioning RPE layer is established. In terms of encouraging differentiation, growth factor 'pockets' that release upon cell contact or PEG acrylate modified growth factors that promote RPE differentiation could be included within the gel. Further work must then be conducted to ensure adequate repair of the adjacent neuronal layer as well as the choricocapillaris in order to restore all tissue layers to their normal, functioning state. 


\section{REFERENCES CITED}

Ablonzsky Z, Dahrouj M, Tang PH, Liu Y, Sambamurti K, Marmorstein AD, Crosson CE. "Human retinal pigment epithelium cells as functional models for the RPE in vivo." Invest Ophthalmol Vis Sci. 2011; 52(12): 8614-8620

Aisenbrey S, Zhang M, Bacher D, Yee J, Brunken WJ, Hunter DD. "Retinal Pigment Epithelial Cells Synthesize Laminins, Including Lamin 5, and Adhere to Them through $\alpha 3$ - and $\alpha 6$-Containing Integrins." Investigative Ophthalmology \& Visual Science 2006. 47(12); 5537-5544.

Avila, MP, Farah ME, Santos A, Duprat JP, Woodward BW, Nau J. "Twelve-month short-term safety and visual-acuity results from a multicentre prospective study of epiretinal strontium-90 brachytherapy with bevacizumab for the treatment of subfoveal choroidal neovascularisation secondary to age-related macular degenerati." The British Journal of Ophthalmology, 2009: 93(3); 305-309.

Binder S, Krebs I, Hilgers R- D, Abri A, Stolba U, Assadoulina A. Outcome of transplantation of autologous retinal pigment epithelium in age-related macular degeneration: a prospective trial. Invest Ophthalmol Vis Sci 2004; 45(11): 4151-60.

Beatty S, Koh H, Phil M, Henson D, Boulton M "The role of oxidative stress in the pathogenesis of age related macular degeneration." Surv Ophthalmol. 2000: 45; 115-134

Blaauwgeers HG, Holtkamp GM, Rutten H, Witmer AN, Koolwijk P, Partanen TA, Alitalo K, Kroon ME, Kijlstra A, van Hinsbergh VW, Schlingemann RO. "Polarized vascular endothelial growth factor secretion by human retinal pigment epithelium and localization of vascular endothelial growth factor receptors on the inner choriocapillaris. Evidence for a trophic paracrine relation." Am J Pathol 1999; 155: 421-428.

Brandl F, Henke M, Rothschenk S, Gschwind R, Breunig M, Blunk T, Tessmar J, Gopferich A. "Poly(EthyleneGlycol) Based Hydrogels for Intraocular Applications." Advanced Engineering Materials 9007: 9(12); 1141-1149.

Browning DJ, Kaiser PK, Rosenfeld PJ, Stewart MW. “Aflibercept for age-related macular degeneration: a game-changer or quiet addition?" Am J Ophthalmol. 2012; 154(2): 222-226.

Burke JM. "Epithelial phenotype and the RPE: Is the answer blowing in the Wnt?" Progress in Retinal and Eye Research. 2008: 579-595. 
de Jong, Paulus. "Mechanisms of Disease: Age-Related Macular Degeneration." The New England Journal of Medicine 2006; 355(14): 1474-1485.

Del Priore LV, Tezel TH. "Reattachment rate of human retinal pigment epithelium to layers of human Bruch's membrane". Arch Ophthalmol 1998; 116(3): 335-41.

Del Priore, LV., Tezel TH, Kaplan HJ. "Maculoplasty for age-related macular degeneration: Reengineering Bruch's membrane and the human macula." Progress in Retinal and Eye Research, 2006: 25:539-562.

Doheny Eye Institute. "The Amsler Grid and Age-related Macular Degeneration" 2007. http://www.doheny.org/patientcare/amsler_grid.pdf; accessed July 23, 2013.

The Eye Diseases Prevalence Research Group. "Prevalence of Age-Related Macular Degeneration in the United States" Archives of Ophthalmology. 2004; 122(4): 564-572.

Feng, Yuezhong, and Milan Mrksich. "The Synergy Peptide PHSRN and the Adhesion Peptide RGD Mediate Cell Adhesion through a Common Mechanism." Biochemistry, 2004; 43:15811-15821.

Forrester JV, Dick AD, McMenamiri PG, Lee WR, The eye: basic sciences in practice. $2^{\text {nd }}$. London: Harcourt Publishers Limited; 2002.

Gibas I, Janik H. "Review: Synthetic Polymer Hydrogels for Biomedical Applications" Chemistry and Chemical Technology. 2010; 4(4); 297-304.

Gobin, Andrea S., and Jennifer L. West. "Cell migration through defined, synthetic extracellular matrix analogues." Journal of the Federation of American Societies for Experimental Biology, 2002.

Gombotz WR, Guanghui W, Horbett TA, Hoffman AS. "Protein adsorption to poly ethylene oxide) surfaces." Journal of Biomedical Materials Research. 1991; 25: $1547-1562$.

Grierson I, Hiscott P, Hogg P, Robey H, Mazure A and Larkin G. "Development, repair and regeneration of the retinal pigment epithelium." Eye, 1994; 8(2): 255262.

Guyton JE, Hall AC. Guyton and Hall Textbook of Medical Physiology, Philadelphia: Saunders, 12 ${ }^{\text {th }}, 2011$. Chapter 50. 
Hahn MS, Taite LJ, Moon JJ, Rowland MC, Ruffino KA, West JL. "Photolithographic patterning of polyethylene glycol hydrogels" Biomaterials. 2006; 27(12): 2519-2524.

Hirano, Y, M Okuno, T Hayashi, K Goto, and A Makajima. "Cell-attachment activities of surface immobilized oligopeptides RGD, RGDS, RGDV, RGDT, and YIGSR toward five cell lines." Journal of Biomaterial Science Polymer Edition, 1993: 235-43.

Hollifield J, Witkovsky P. "Pigmented retinal epithelium involvement in photoreceptor development and function.” Exp ZooI. 1974; 189: 357-77.

Hudspeth AJ, Yee AG. "The intercellular junctional complexes of retinal pigment epithelia." Invest Ophthalmol 1973; 12:354-65.

Ito Y, Kajihara M, Imanishi Y. "Materials for enhancing cell adhesion by immobilization of cell-adhesive peptide." Journal of Biomedical Material Research. 1991; 25(11): 1325-37.

Jiang, LQ, Jorquera M, Streilein JW, "Immunologic consequences of intraocular implantation of retinal pigment epithelial allografts." Exp. Eye Res. 1994; 58(6): 719-728.

Kaplan HJ, Del Priore LV, Tezel TH, Berger AS. "Retinal Pigment Epithelial Transplantation for Subfoveal Neovascularization in Age-related Macular Degeneration: a Clinical Trial." Washington, DC. Abstract presented as the Annual Meeting of The Retina Society

Karp, Gerald. Cell and Molecular Biology. Atlantic Highlands: John Wiley \& Sons, Inc., 2008.

Kubota A, Nishida K, Yamato M, Yang J, Kikuchi A, Okano T, Tano Y. "Transplantable retinal pigment epithelial cell sheets for tissue engineering." Biomaterials, 2006; 27(19): 3639-3644.

Larson Eye Center. "Human Eye Anatomy", 2012. http://www.larsoneyecenter.com/ chicago/images/anatomy-of-the-eye.gif; accessed February 2, 2013.

Lee CJ, Fishman HA, Bent SF. "Spatial cues for the enhancement of retinal pigment epithelial cell function in potential transplants." Biomaterials, 2007: 28; 21922201

Lee CJ, Vroom JA, Fishman HA, Bent SF. "Determination of human lens capsule permeability and its feasibility as a replacement for Bruch's membrane." Biomaterials. 2006; 27: 1670-1678. 
Li H, Wang H, Wang F, Gu Q, Xu X. "Snail Involves in the Transforming Growth Factor beta 1-Mediated Epithelial-Mesenchymal Transition of Retinal Pigment Epithelial Cells" PLoS One. 2011; 6(8): e23322.

Lu L, Yaszemski MJ, Mikos AG. "Retinal pigment epithelium engineering using synthetic biodegradable polymers." Biomaterials, 2001: 3345-3355.

Luthert, PJ. "Pathogenesis of age-related macular degeneration". Diagnostic Histopathology, 2011; 17(1): 10-16.

Mann BK, Gobin AS, Tsai AT, Schmedlen RH, West JL. "Smooth muscle cell growth in photopolymerized hydrogels with cell adhesive and proteolytically degradable domains: synthetic ECM analogs for tissue engineering". Biomaterials. 2001; 22: 3045-3051.

Marshall J, Hussain AA, Starita C, Moore DJ, Patmore AL. Aging and Bruch's membrane. In: Marmor MF, Wolfensberger TJ, editors. The retinal pigment epithelium: function and disease. Oxford: Oxford University Press; 1998.

McKay BS, Irving PE, Skumatz CM, Burke JM, "Cell-cell adhesion molecules and the development of an epithelial phenotype in cultured human retinal pigment epithelial cells." Exp. Eye Res. 1997; 65: 661-671.

Mecklenburg, Lars, and Ulrich Schraermeyer. "An Overview on the Toxic Morphological Changes in the Retinal Pigment Epithelium after Systemic Compound Administration." Toxicologic Pathology, 2007; 35: 252-267.

Miyajima-Uchida H, Hayashi H, Beppu R, Kuroki M, Fukami M, Arakawa F, Tomita Y, Kuroki M, Oshima K. "Production and accumulation of thrombospondin- 1 in human retinal pigment epithelial cells." Invest Ophthalmol Vis Sci 2000; 41: 561-567.

Moon JJ, Hahn MS, Kim I, Nsiah BS, West JL.”Micropatterning of Poly(Ethylene Glycol) Diacrylate Hydrogels with Biomolecules to Regulate and Guide Endothelial Morphogenesis" Tissue Engineering. 2009; 15(3): 579-585.

National Eye Institute, "Facts About Age-Related Macular Degeneration”, September 2009. http://www.nei.nih.gov/health/maculardegen/armd_facts.asp; accessed February 2, 2013.

National Eye Institute, “Age-Related Macular Degeneration”, February 2011. http://report.nih.gov/NIHfactsheets/ViewFactSheet.aspx?csid=69; accessed February 2, 2013.

Nowak, Jerzy Z. "Age-related macular degeneration (AMD): pathogenesis and therapy." Pharmacological Reports 2006; 58; 353-363. 
Ohno-Matsui K, Morita I, Tombran-Tink J, Mrazek D, Onodera M, Uetama T, Hayano M, Murota SI, Mochizuki M. "Novel mechanism for age-related macular degeneration: an equilibrium shift between the angiogenesis factors VEGF and PEDF." J Cell Physiol 2001; 189: 323-333.

Ortega-Velázquez R, Díez-Marqués ML, Ruiz-Torres MP, González-Rubio M, Rodríguez-Puyol M, Rodríguez Puyol D. “Arg-Gly-Asp-Ser (RGDS) peptide stimulates transforming growth factor beta1 transcription and secretion through integrin activation." Journal of the Federation of American Societies for Experimental Biology. 2003. 17(11):1529-31.

Ozden S, Barron AR. "Molecular Weight of Polymers" 2012. http://cnx.org/content/m43550/1.1/; accessed July 24, 2013.

Roorda, Austin, Yubua Zhang, and Jacque L. Duncan. "High-Resolution In Vivo Imaging of the RPE Mosaic in Eyes with Retinal Disease." Investigative Ophthalmology \& Visual Science 2007; 48(5): 2297-2303.

Rosenfeld PJ, Brown DM, Heier JS, Boyer DS, Kaiser PK, Chung CY, Kim RY. "Ranibizumab for Neovascular Age-Related Macular Degeneration" N Engl J Med. 2006; 355:1419-143

Sandig M, Kalnins VI. "Subunits in zonulae adhaerentes and striations in the associated circumferential microfilament bundles in chicken retinal pigment epithelial cells in situ." Exp Cell Res 1988; 175: 1-14.

Scherzer, CM "Evaluation of the effect on cellular attachment and morphology caused by different attachment factors for RPE cells on PEG hydrogels." Unpublished master's thesis, University of Louisville, Louisville, Kentucky. 2011.

Shier D, Butler J, Lewis R. Hole's Essentials of Human Anatomy and Physiology. $11^{\text {th }}$, New York: McGraw-Hill; 2012.

Sheridan C, Williams R, Grierson I. "Basement membranes and artificial substrates in cell transplantation”. Graefe's Arch Clin Exp Opthalmol 2004; 242: 68-75

Sun H, Nathans J. "The Challenge of Macular Degeneration". Scientific American 2001; 285(4): 68-75

Weldon, S. “The Retinal Pigment Epithelium in Visual Function.” 2007. http://www.ami.org/meetings/2007/pressroom/WeldonS.jpg; accessed July 16, 2013.

Zarbin Ma. "Age-related macular degeneration: review of pathogenesis." Eur J Ophthalmol 1998; 8(4): 199-206. 
APPENDIX I: Gel Concentration Quantification Images

All images follow the same naming format:

Gel-(Concentration)-(Gel Number)-(Image Number)

All quantification images are taken at 60x. 


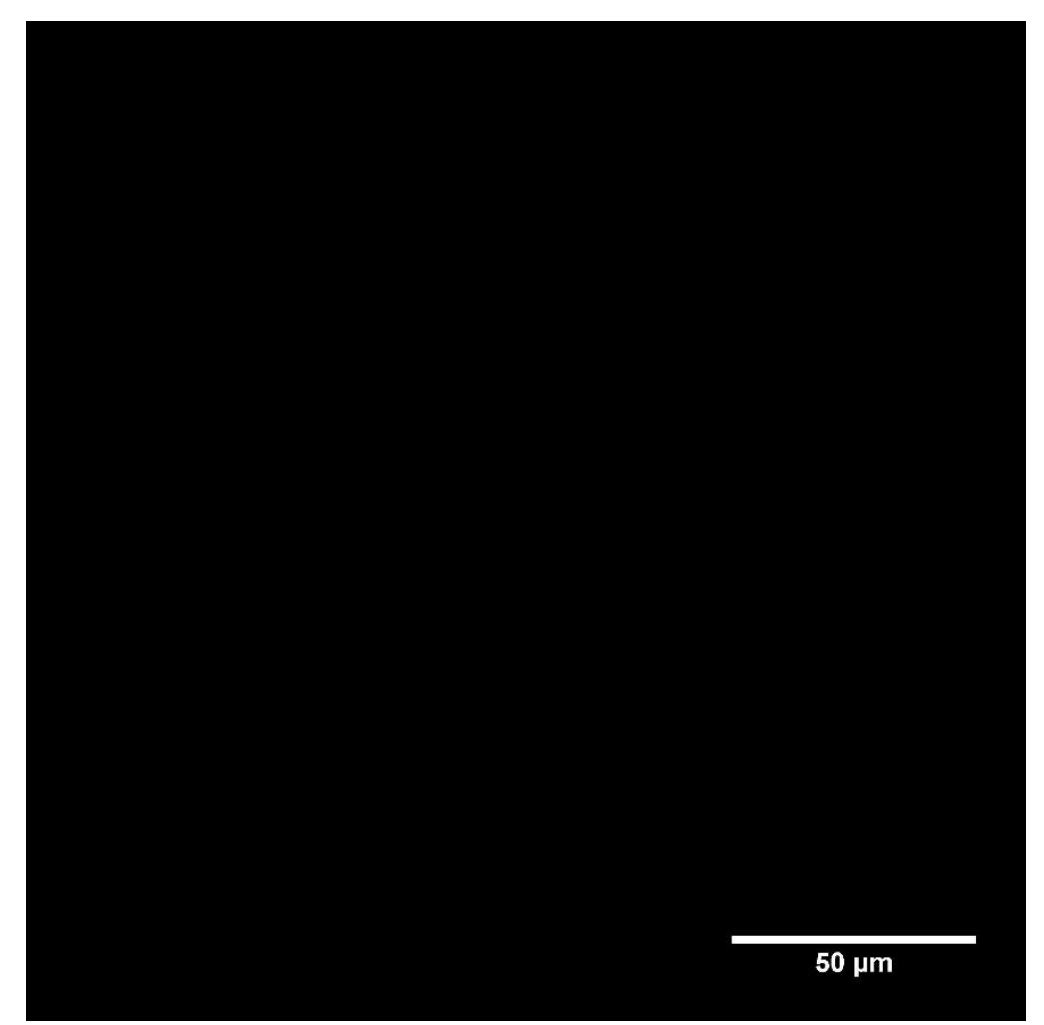

FIGURE A1.1- Gel 0-1-1

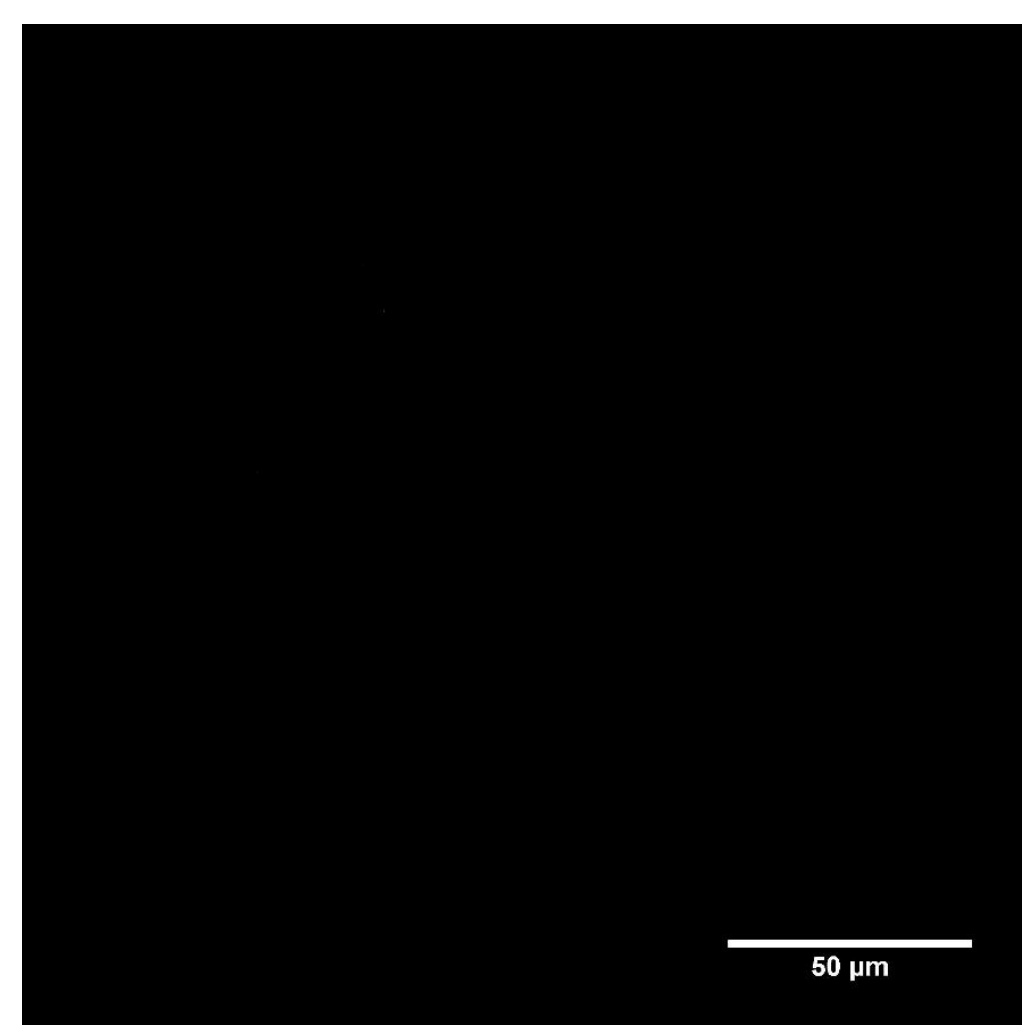

FIGURE A1.2- Gel 0-2-1 


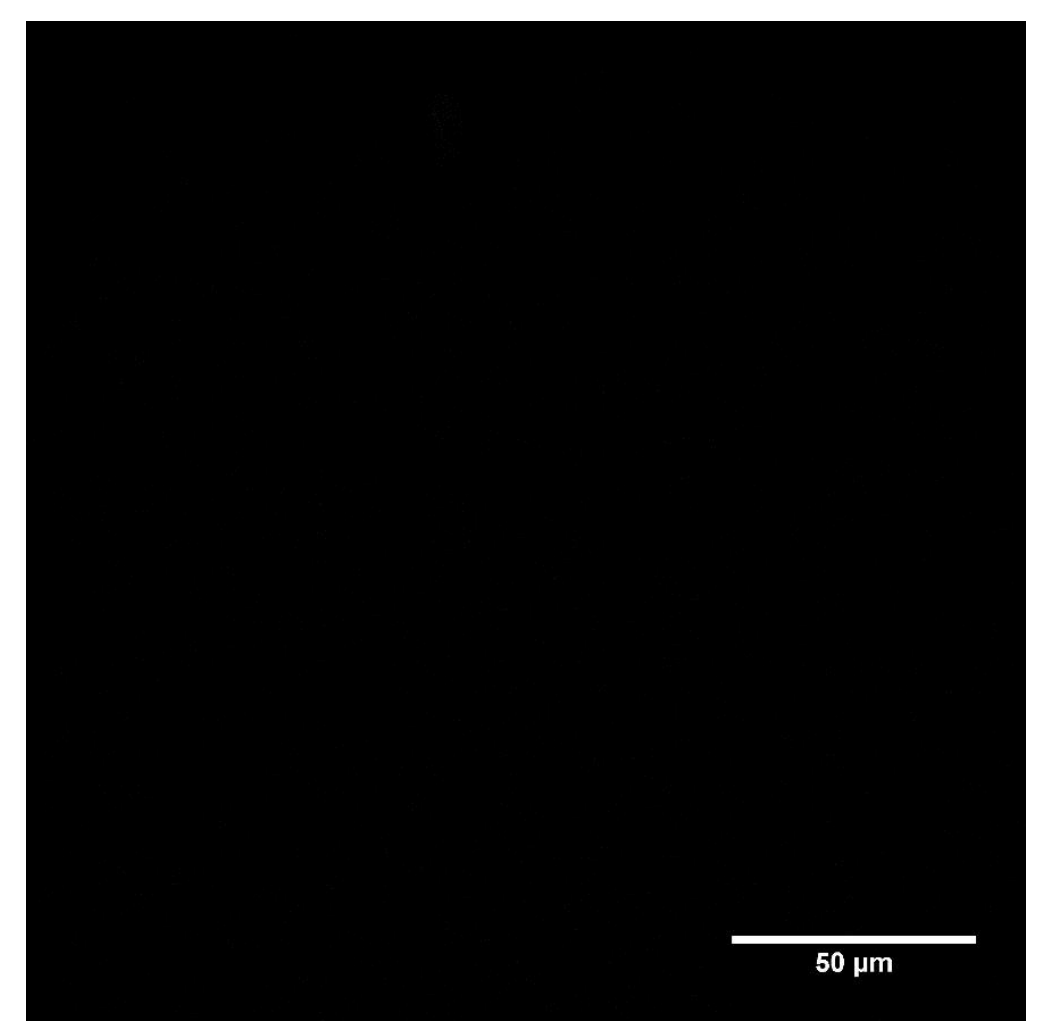

FIGURE A1.3- Gel 0-3-1

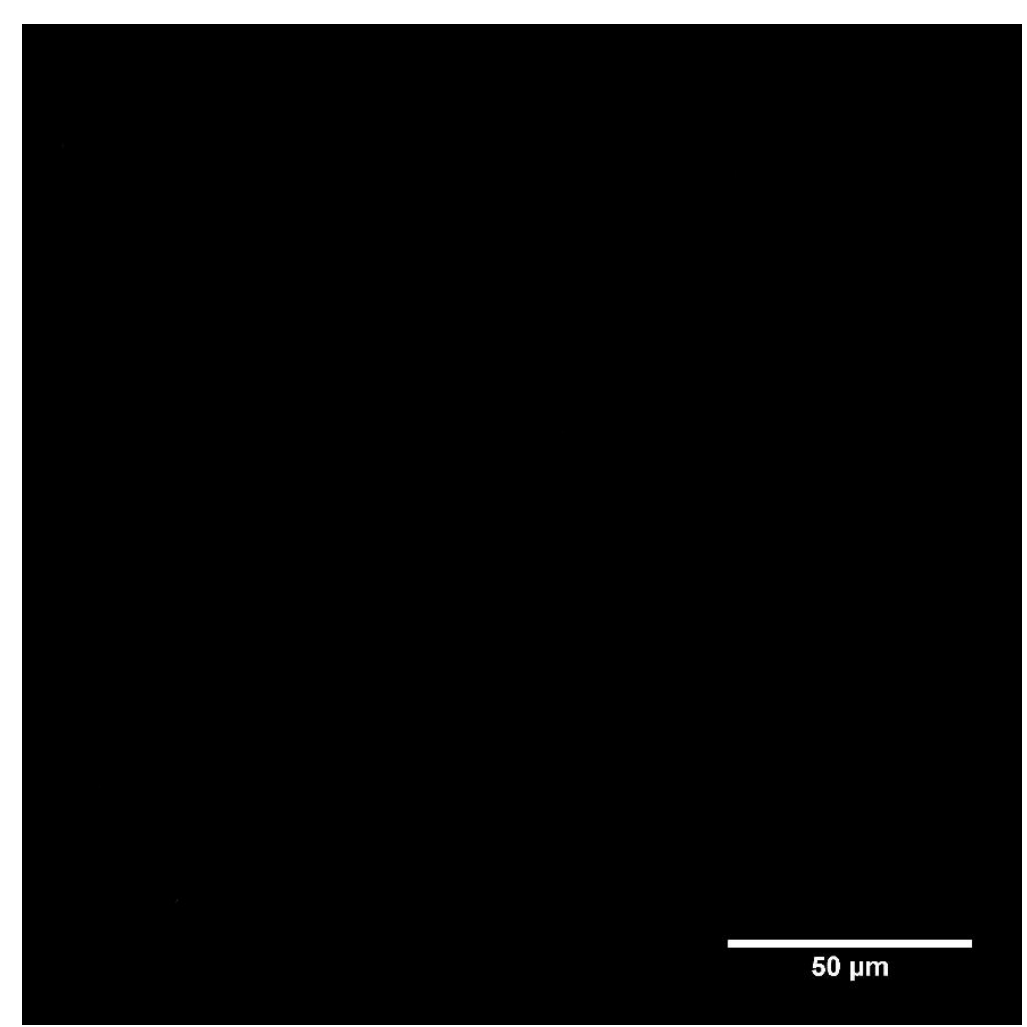

FIGURE A1.4- Gel 0-4-1 


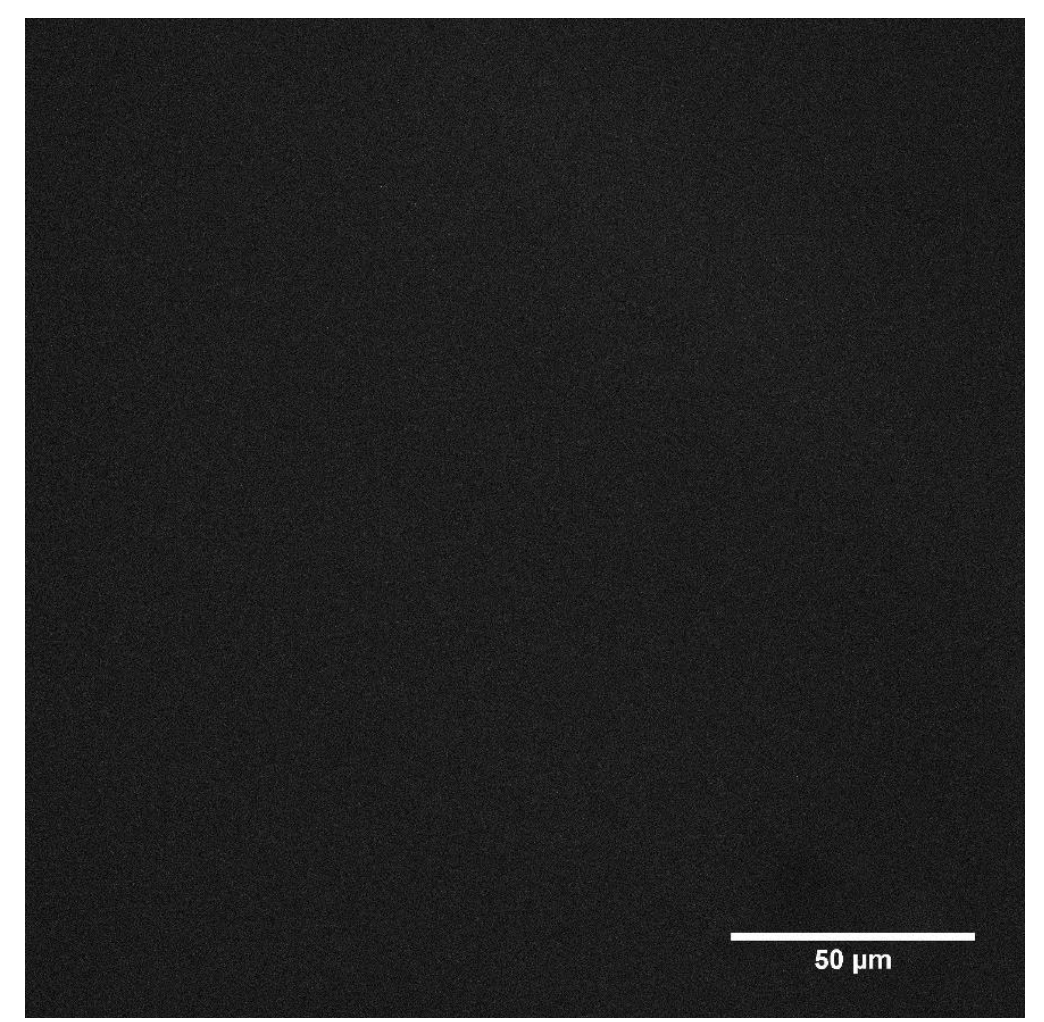

FIGURE A1.5- Gel 5-1-1

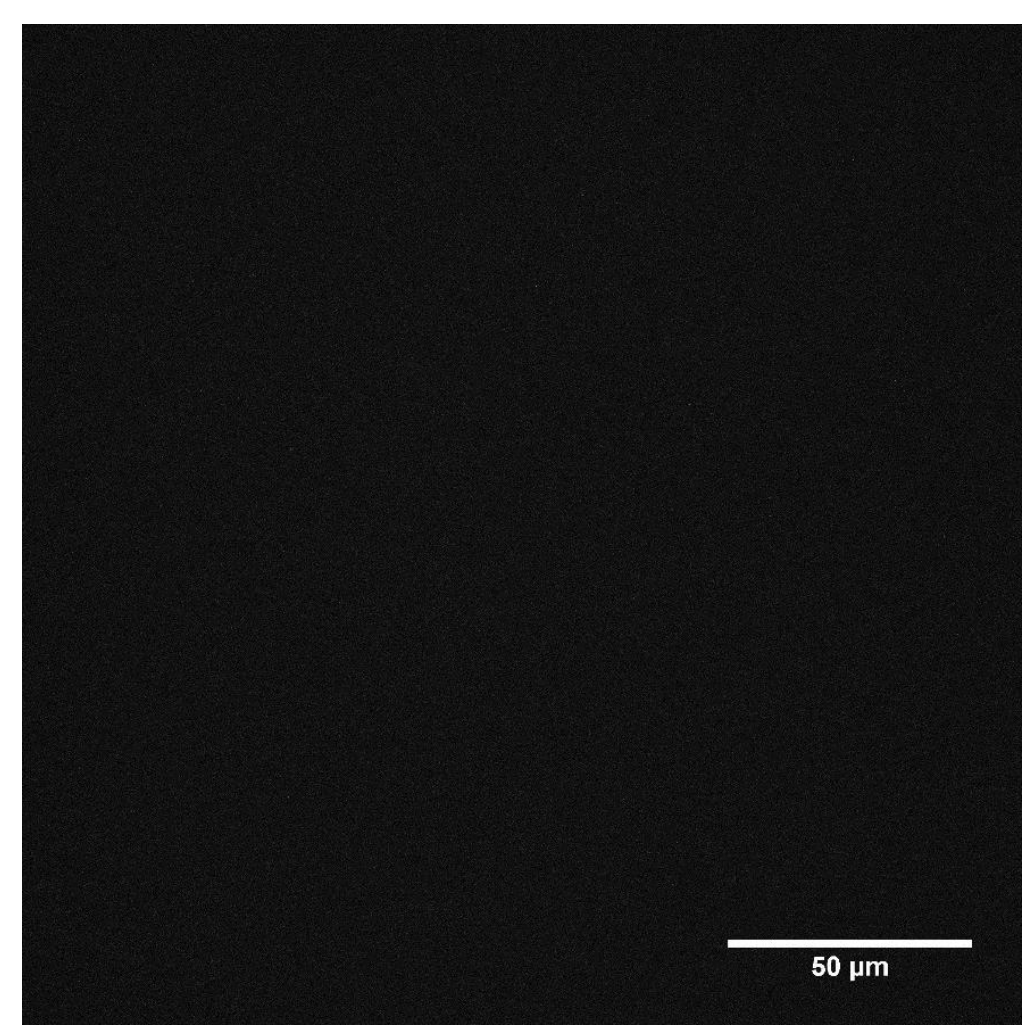

FIGURE A1.6- Gel 5-2-1 


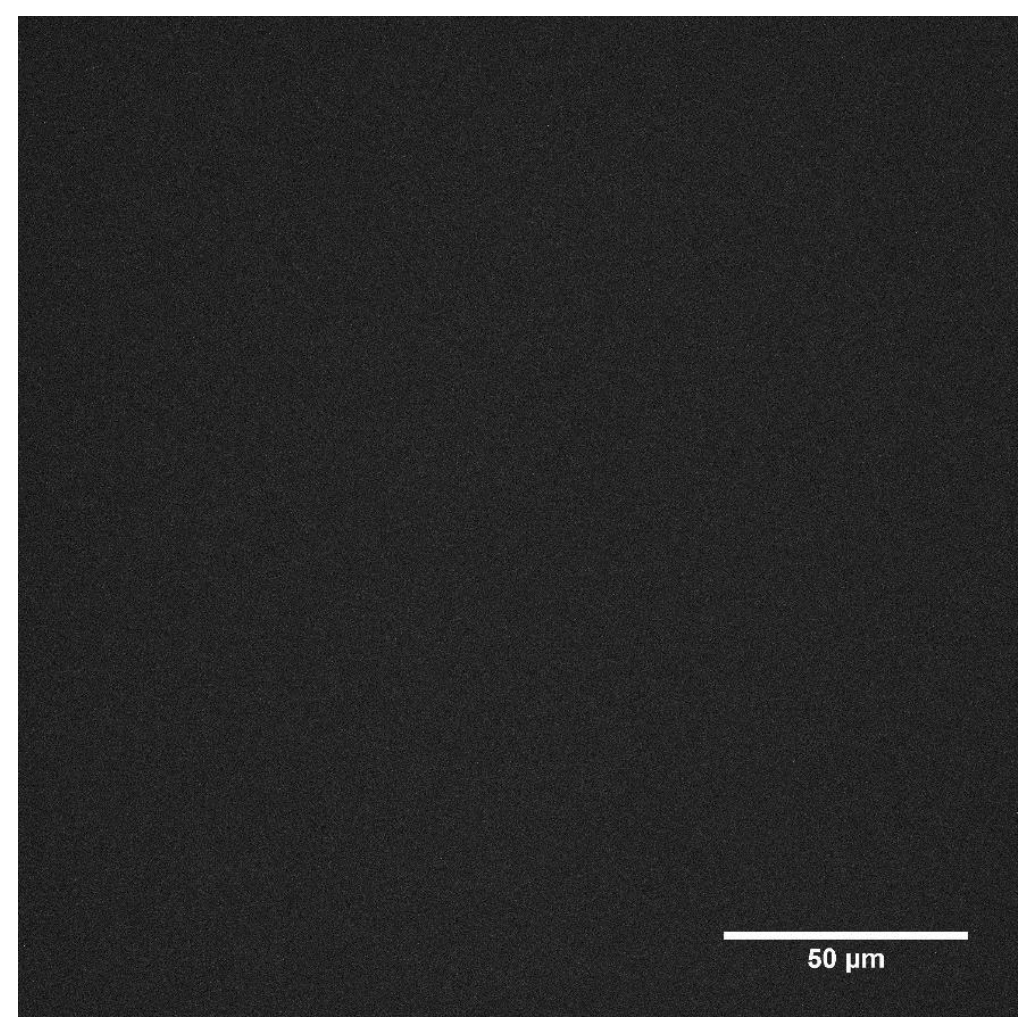

FIGURE A1.8- Gel 5-4-1 


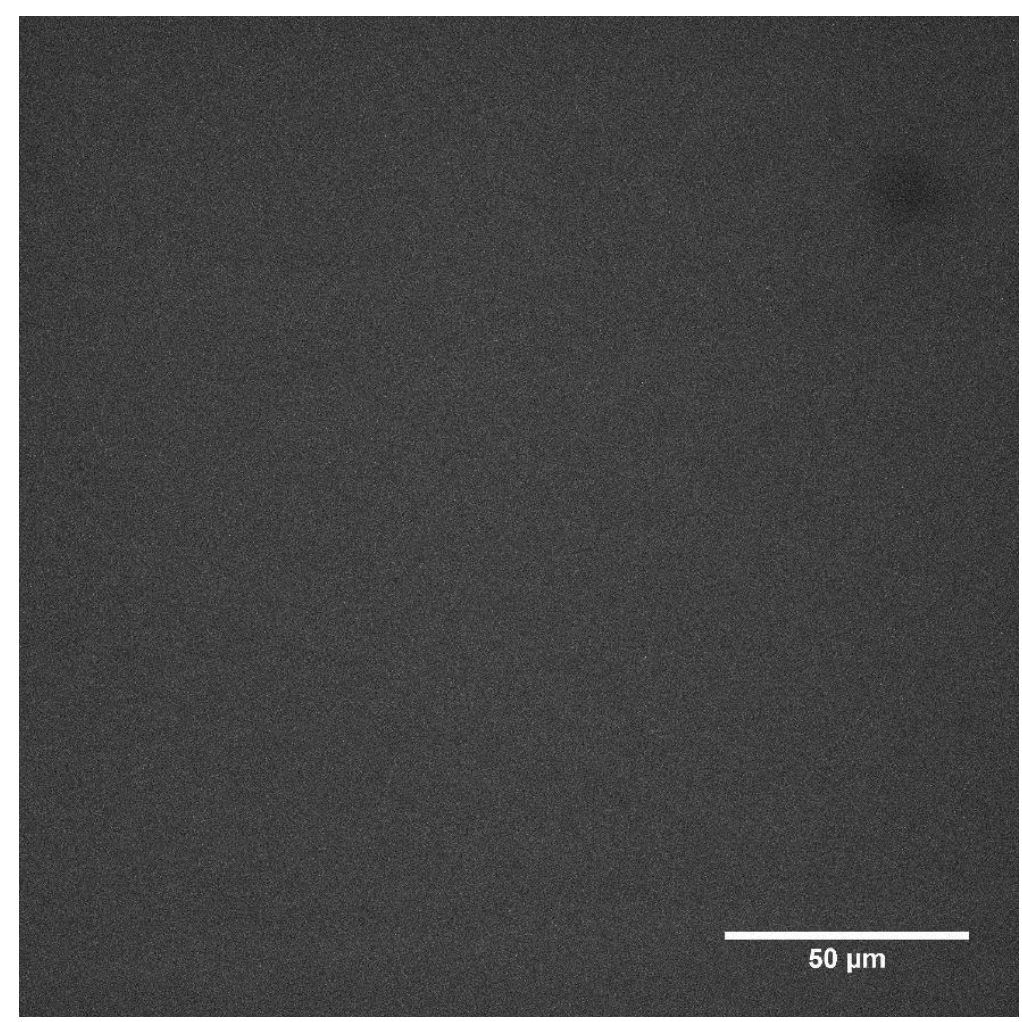

FIGURE A1.10- Gel 10-2-1 


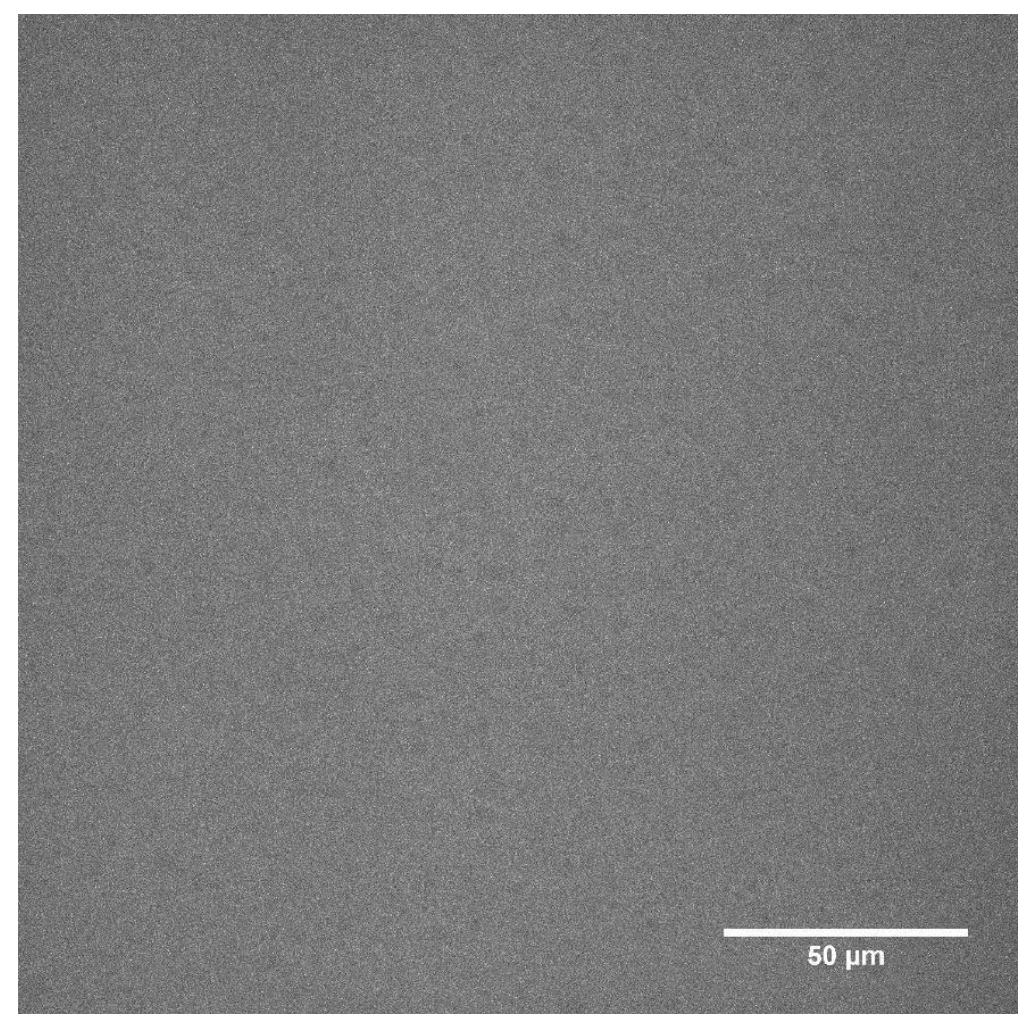

FIGURE A1.11- Gel 10-3-1

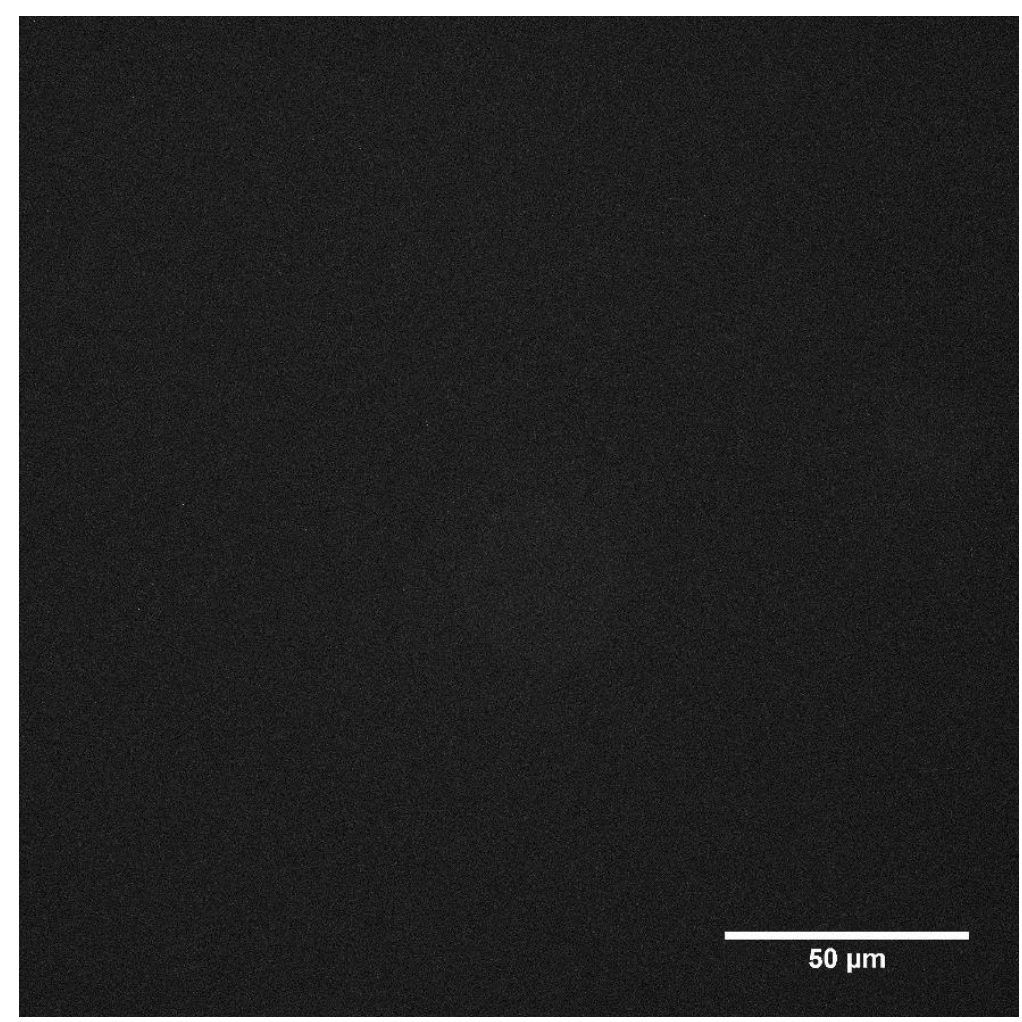

FIGURE A1.12- Gel 10-4-1 


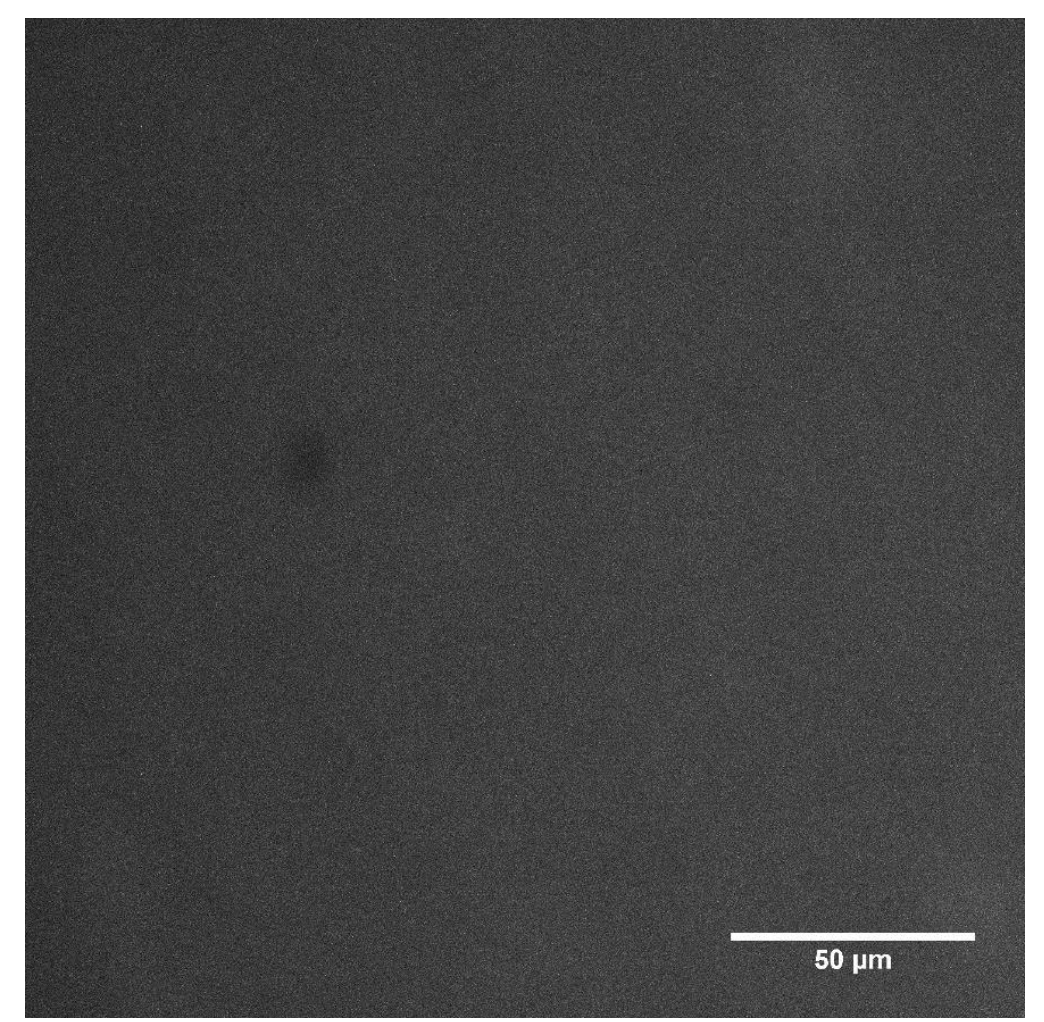

FIGURE A1.13- Gel 20-1-1

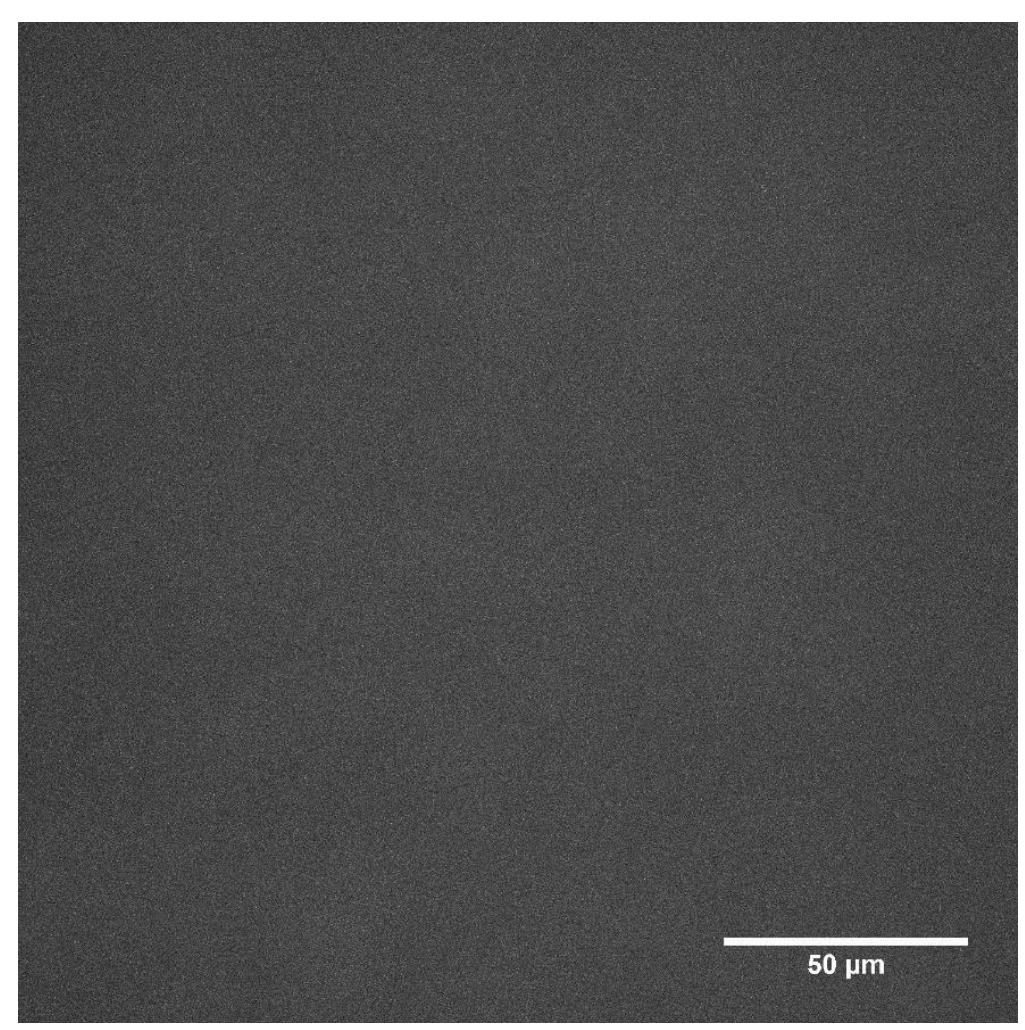

FIGURE A1.14- Gel 20-2-1 


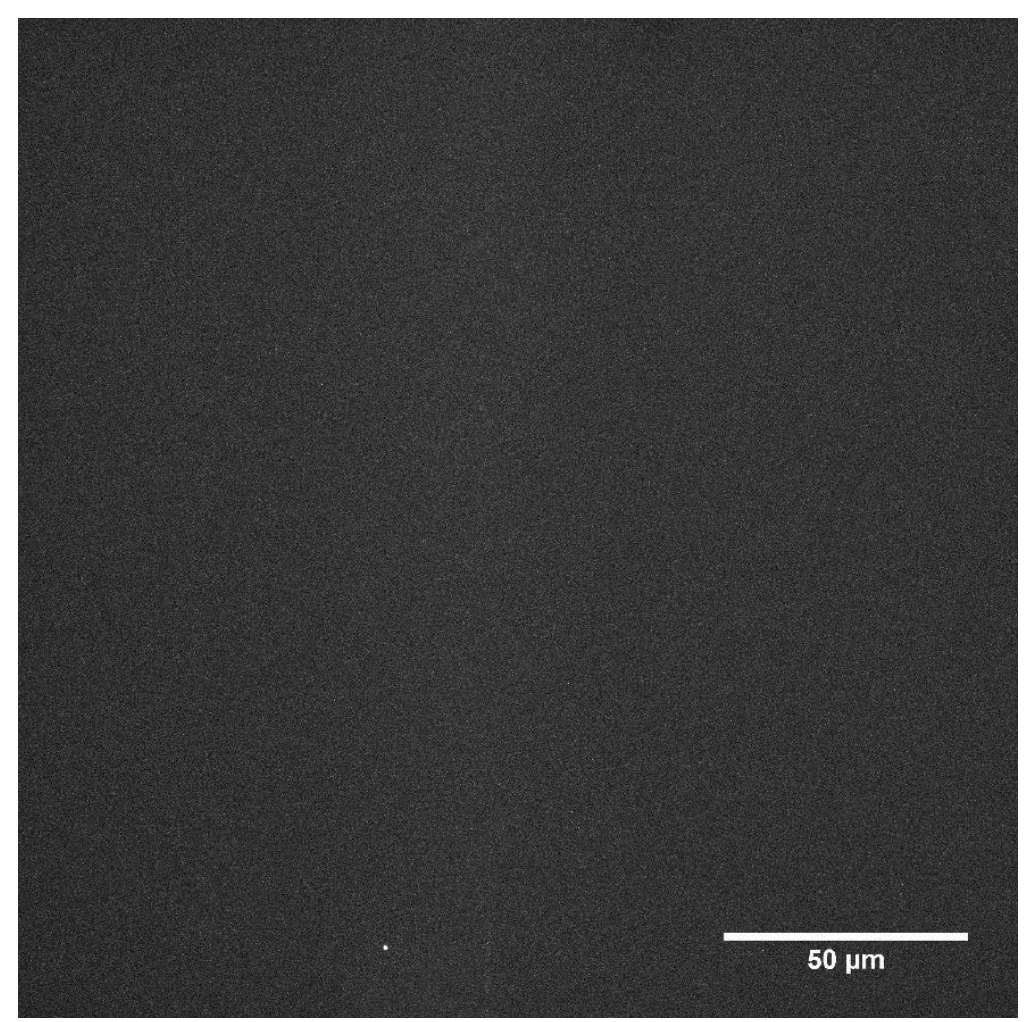

FIGURE A1.15- Gel 20-3-1

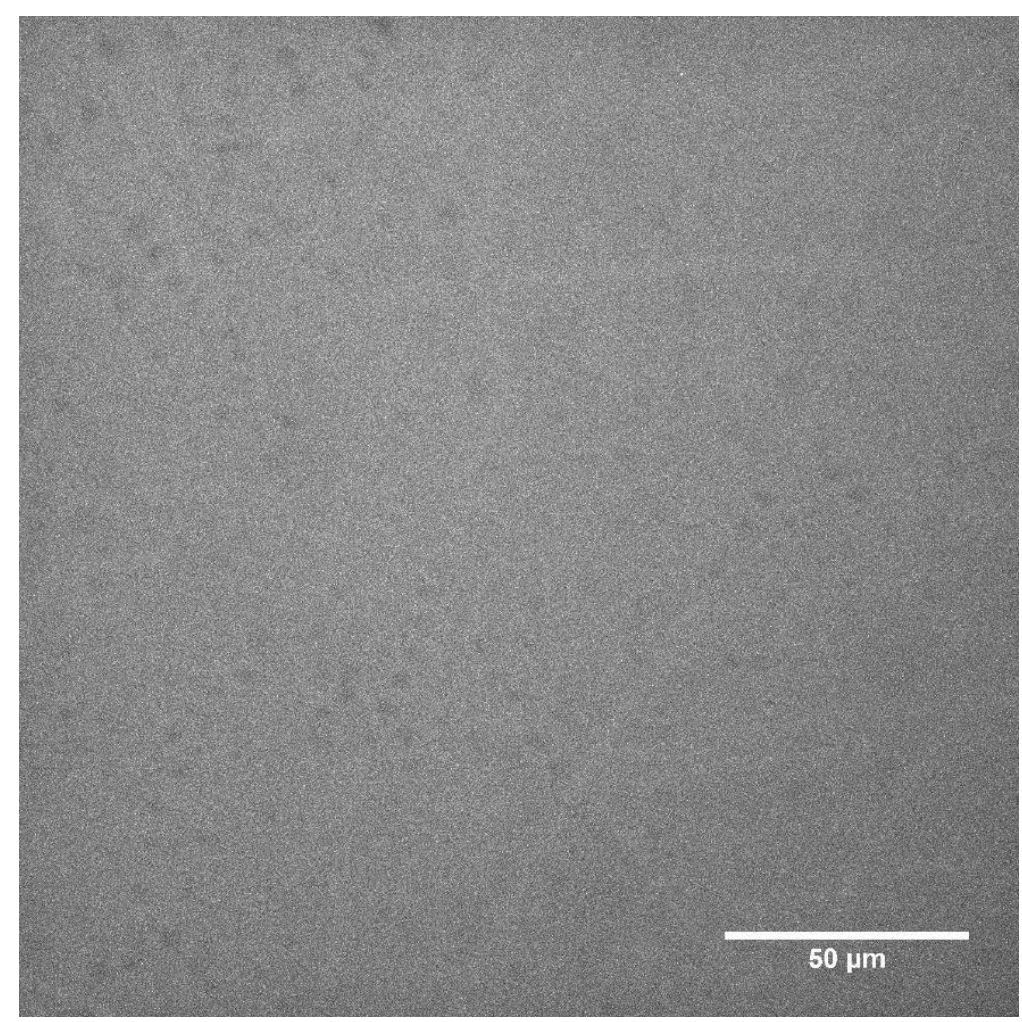

FIGURE A1.16- Gel 20-4-1 


\title{
APPENDIX II: Gel Confluency Images
}

\author{
All images follow the same naming format: \\ (Cell Type) (Concentration)-(Gel Number)-(Image Number)
}

All quantification images are taken at 60x.

Solid black images represent complete (100\%) cellular coverage. 


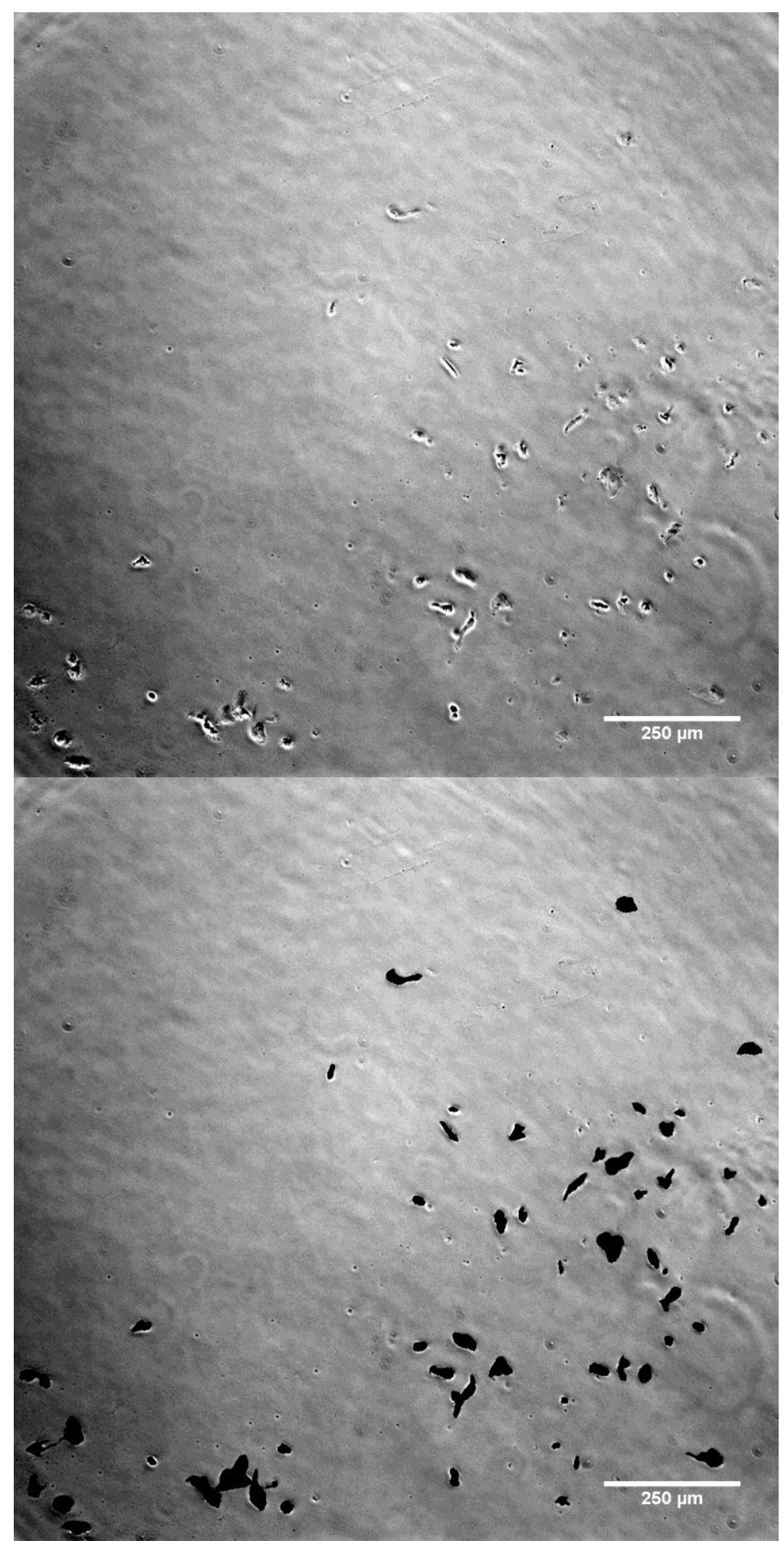

FIGURE A2.1- ARPE 0-1-1 Confluency 


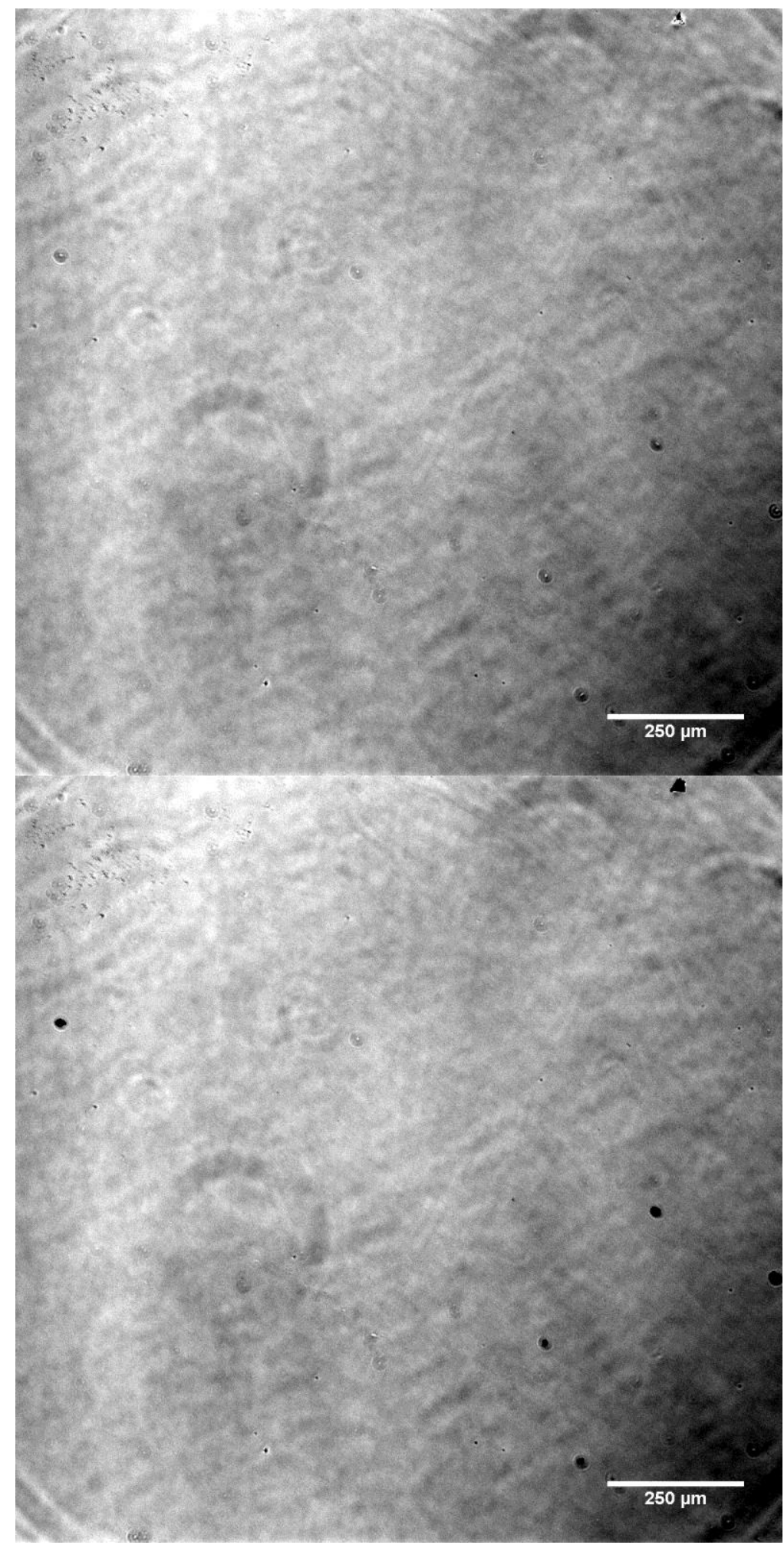

FIGURE A2.2- ARPE 0-1-2 Confluency 


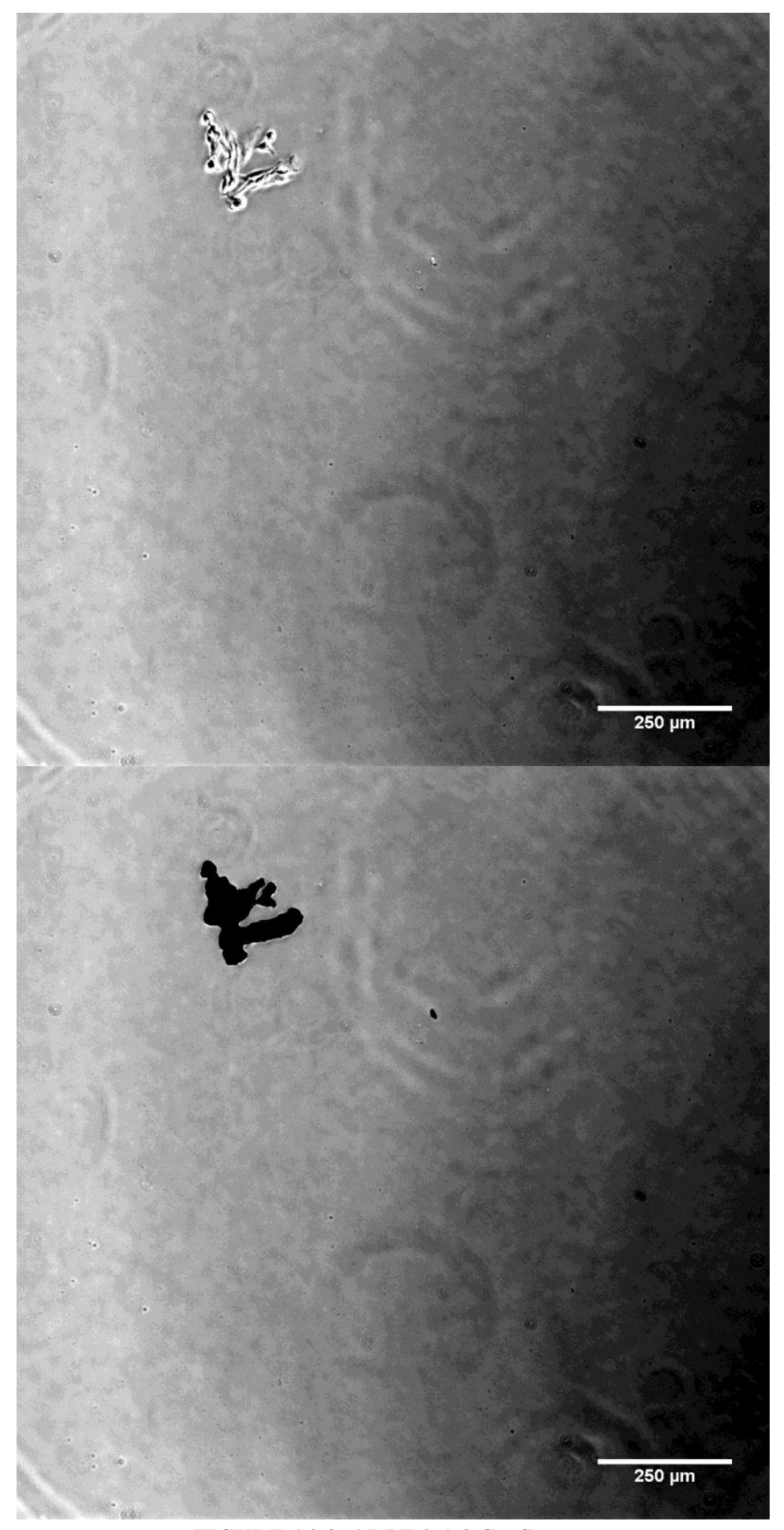

FIGURE A2.3- ARPE 0-1-3 Confluency 


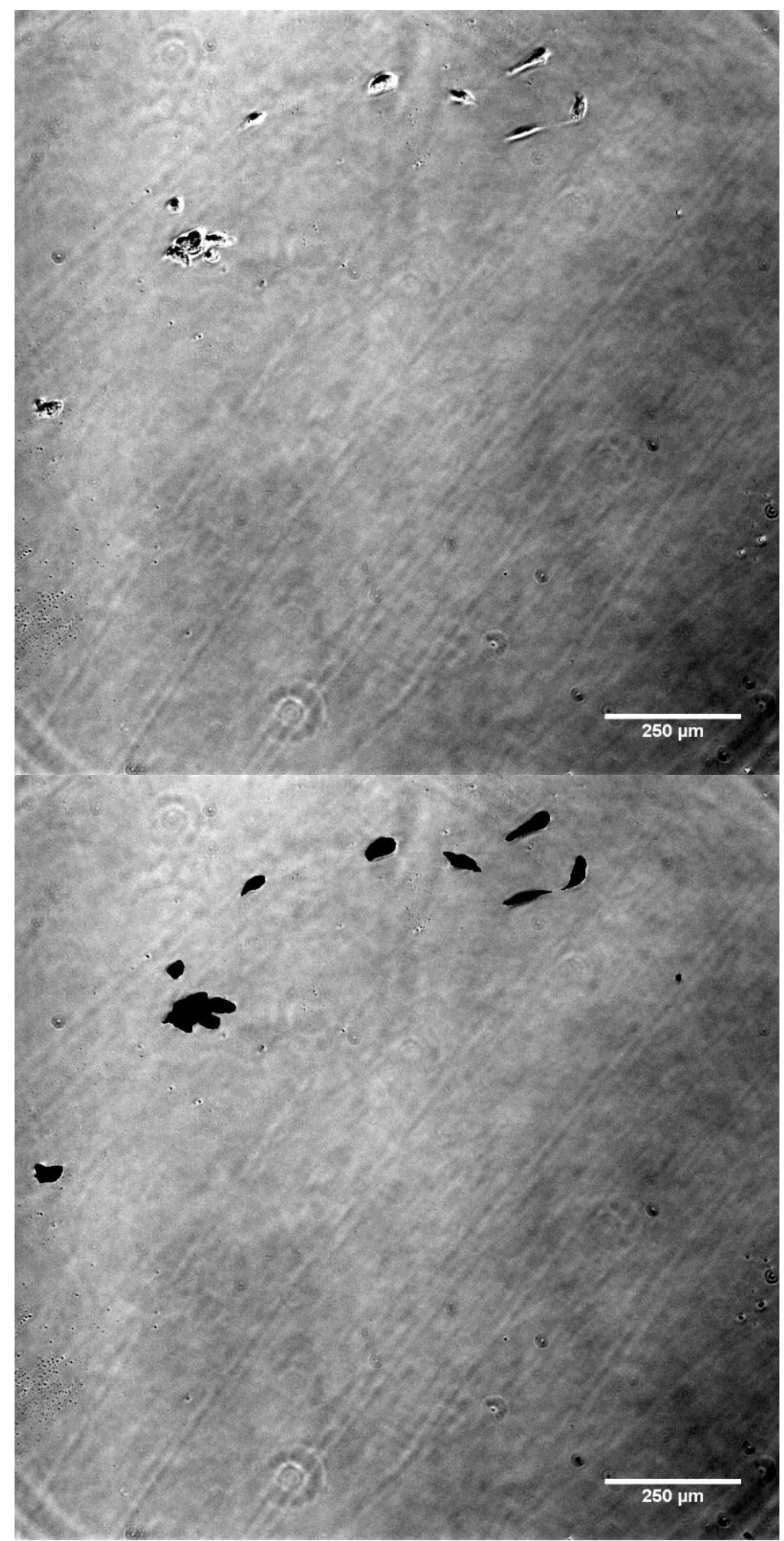

FIGURE A2.4- ARPE 0-2-1 Confluency 


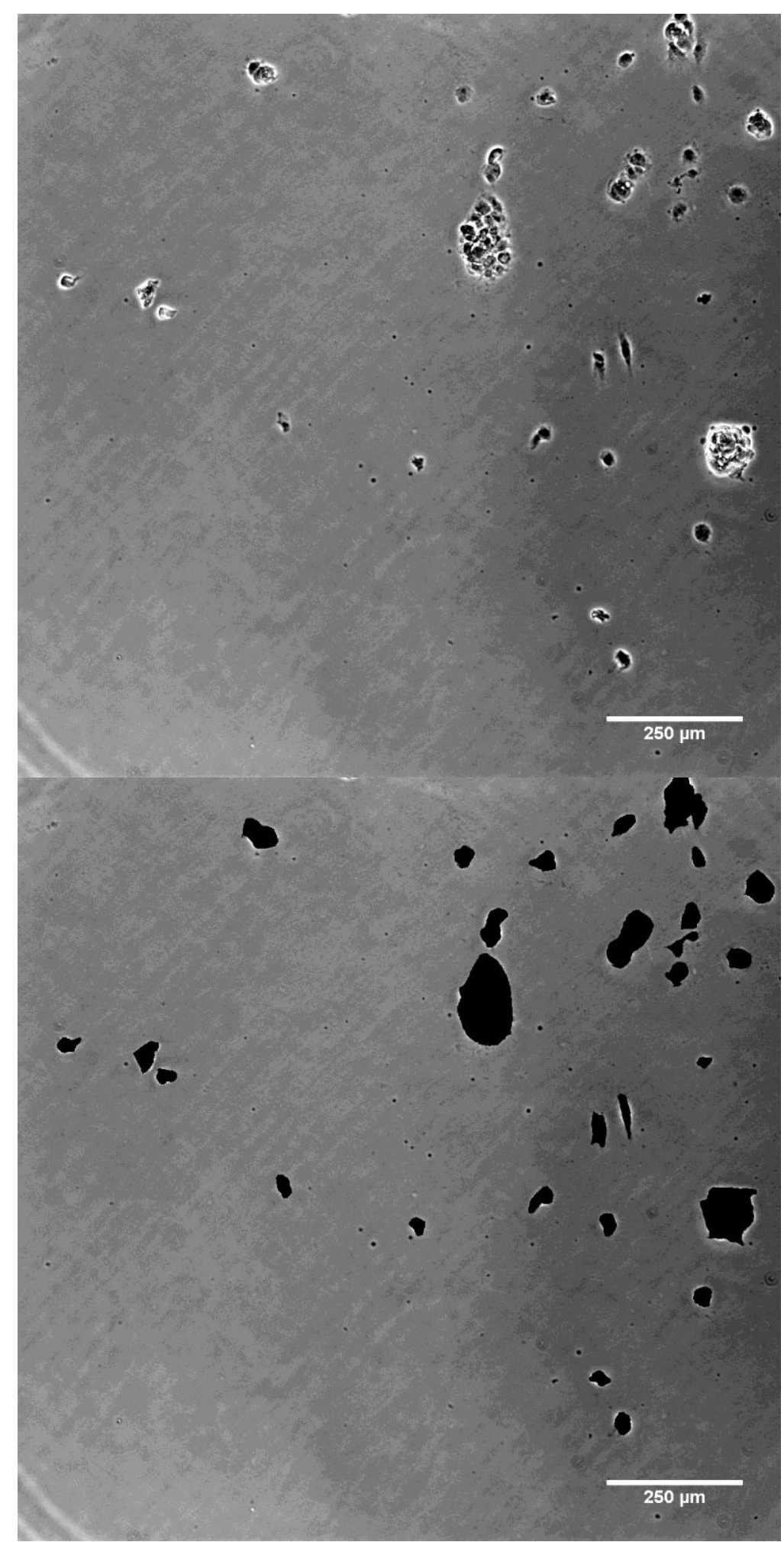

FIGURE A2.5- ARPE 0-2-2 Confluency 


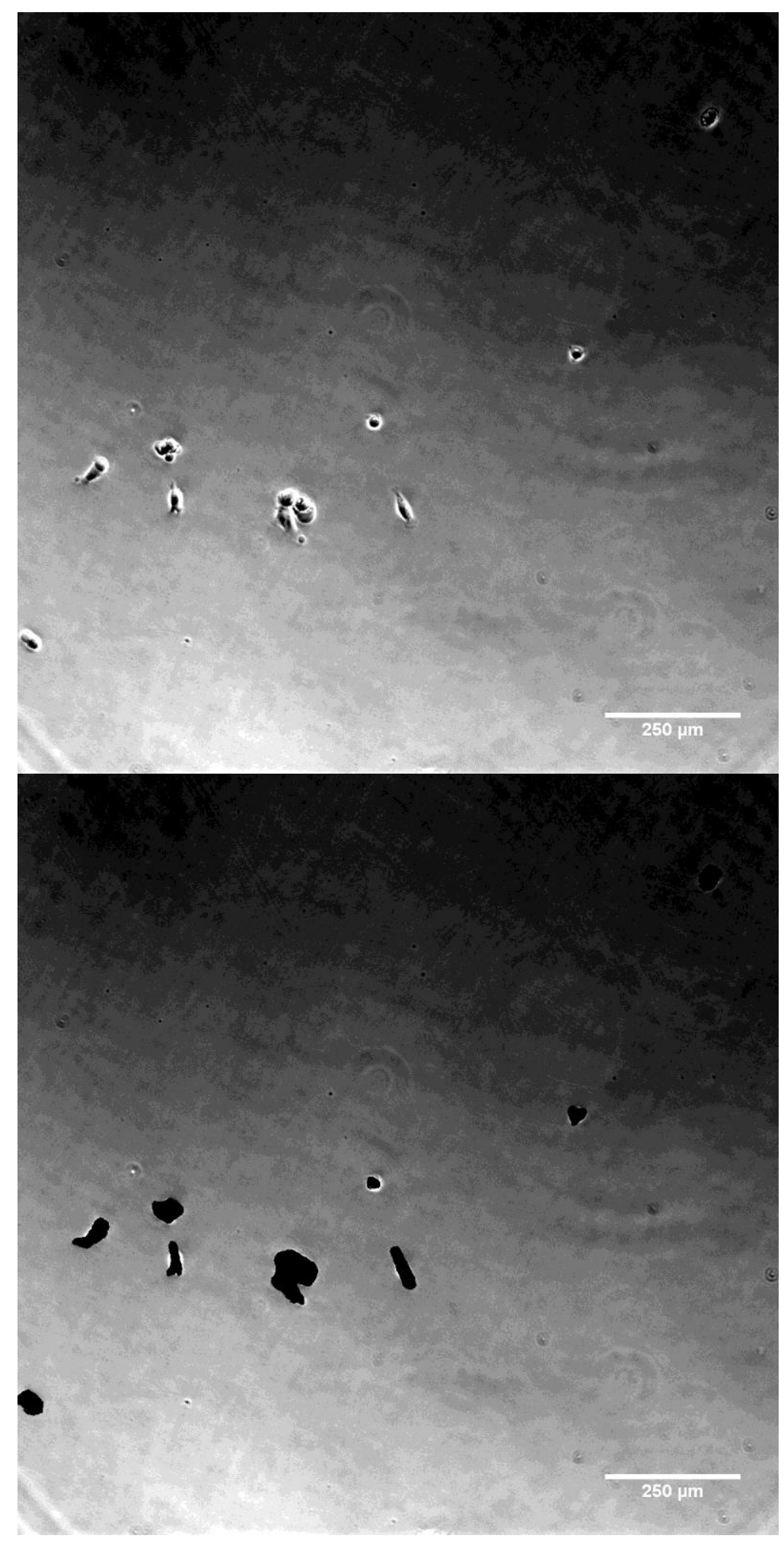

FIGURE A2.6- ARPE 0-2-3 Confluency 


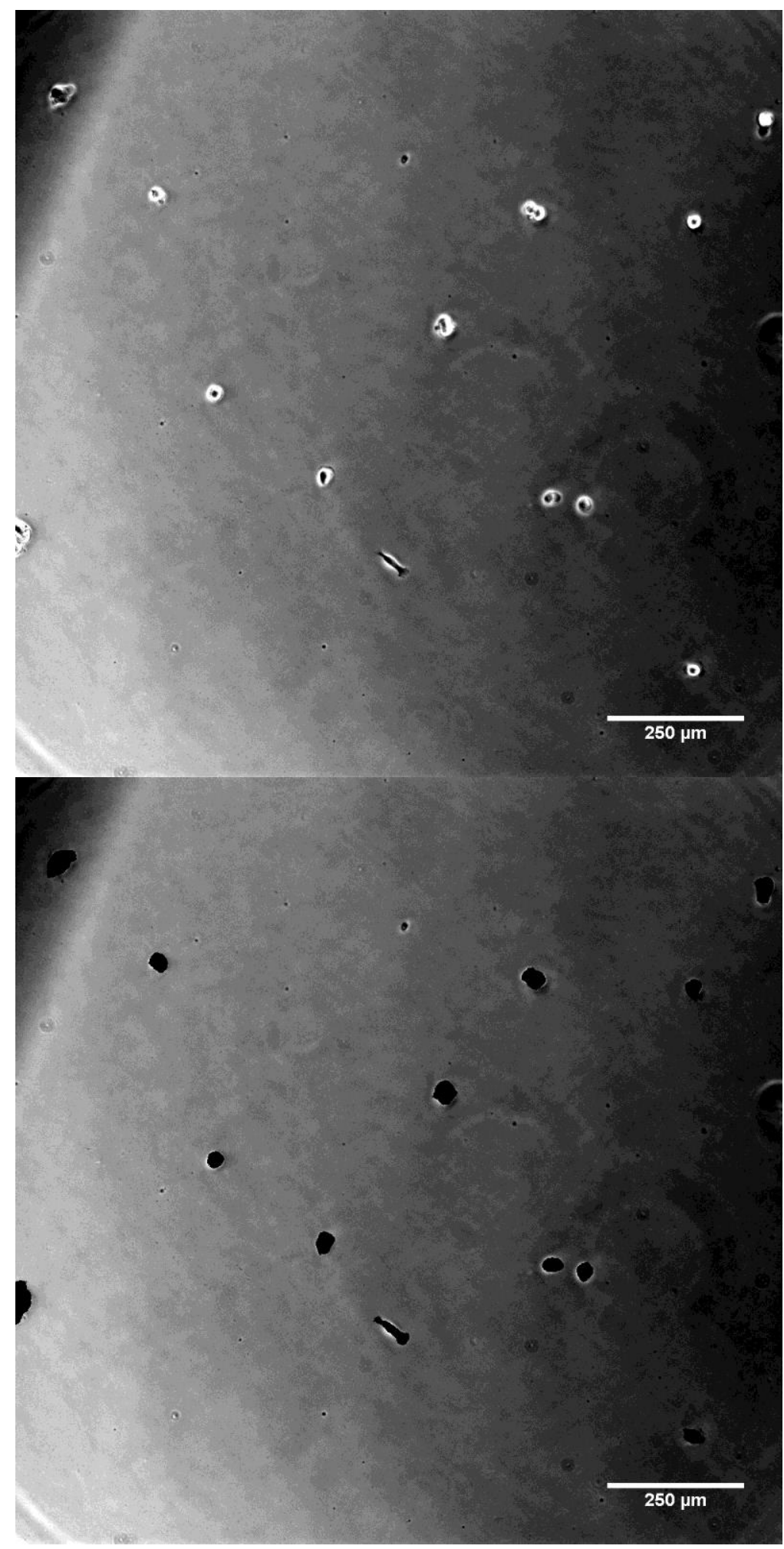

FIGURE A2.7- ARPE 0-3-1 Confluency 


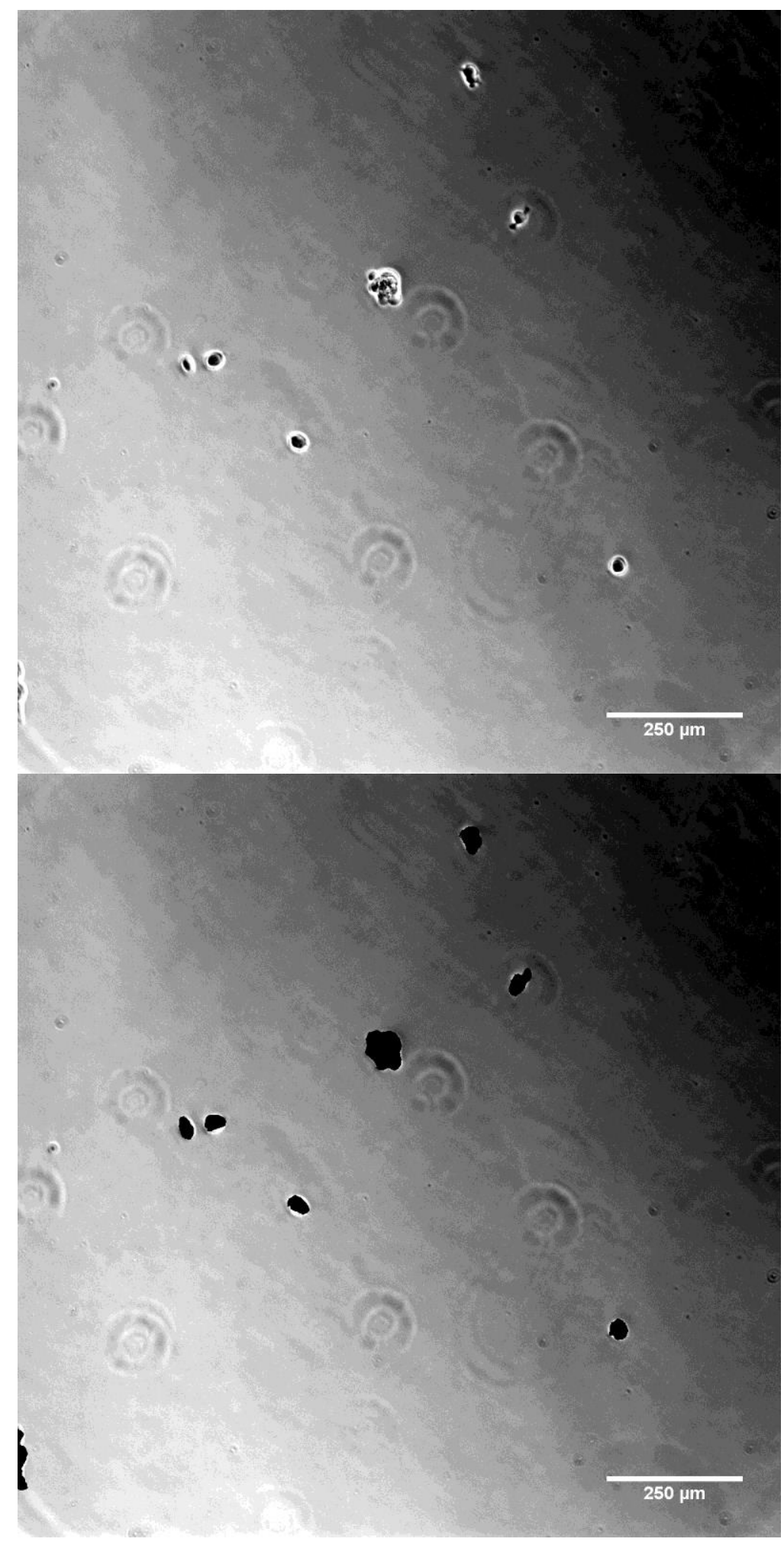

FIGURE A2.8- ARPE 0-3-2 Confluency 


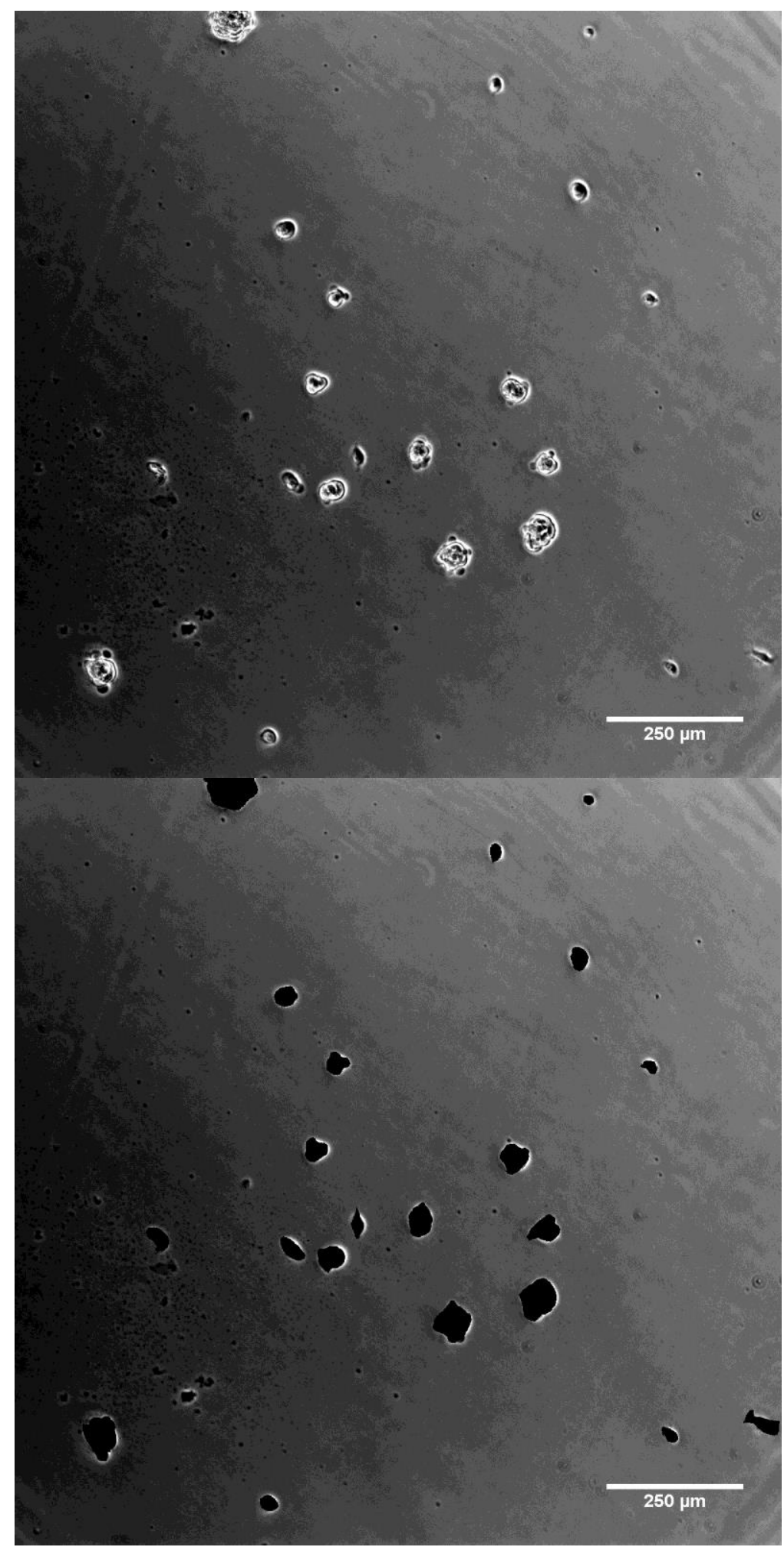

FIGURE A2.9- ARPE 0-3-3 Confluency 


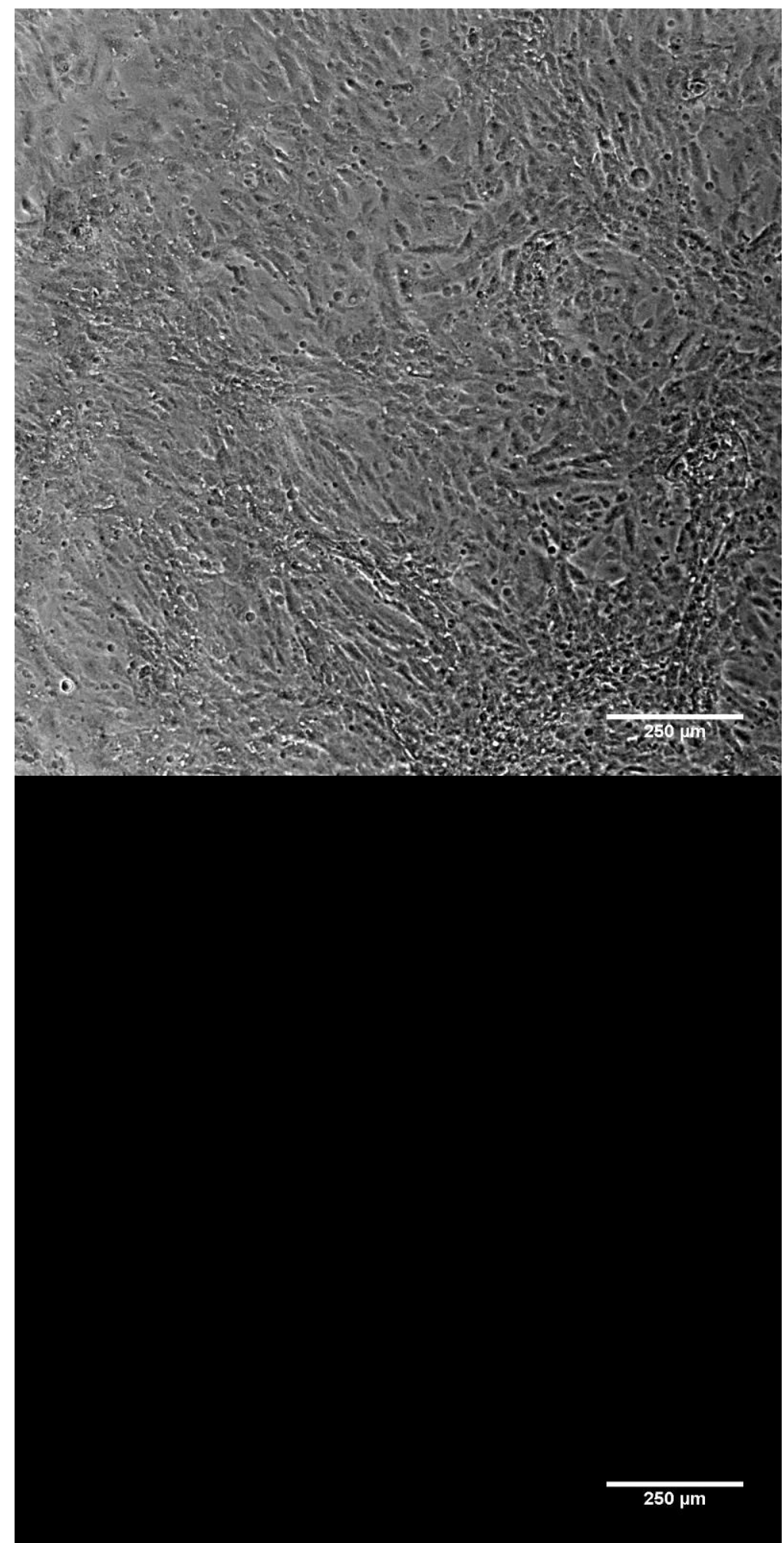

FIGURE A2.10- ARPE 5-1-1 Confluency 


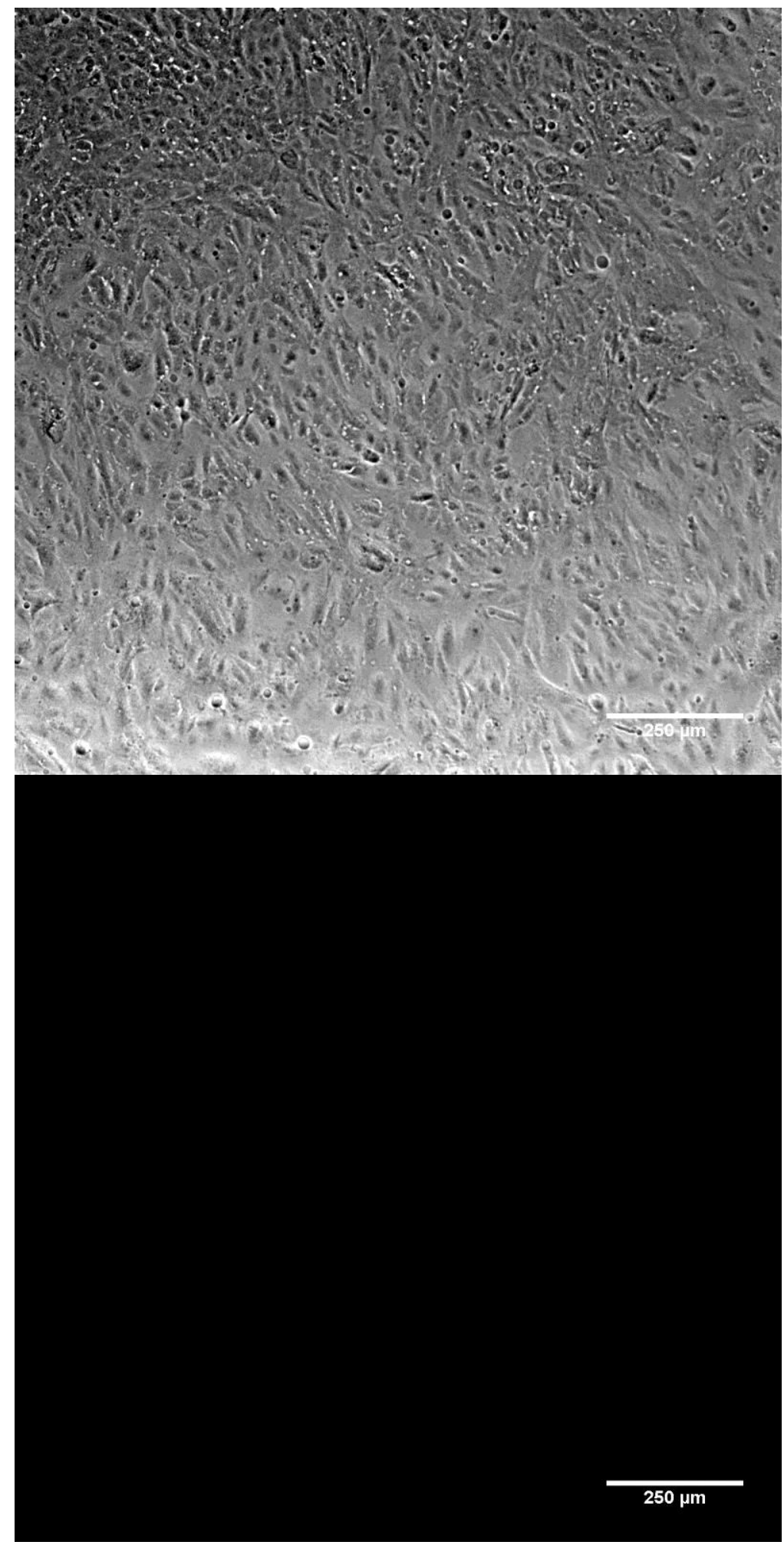

FIGURE A2.11- ARPE 5-1-2 Confluency 


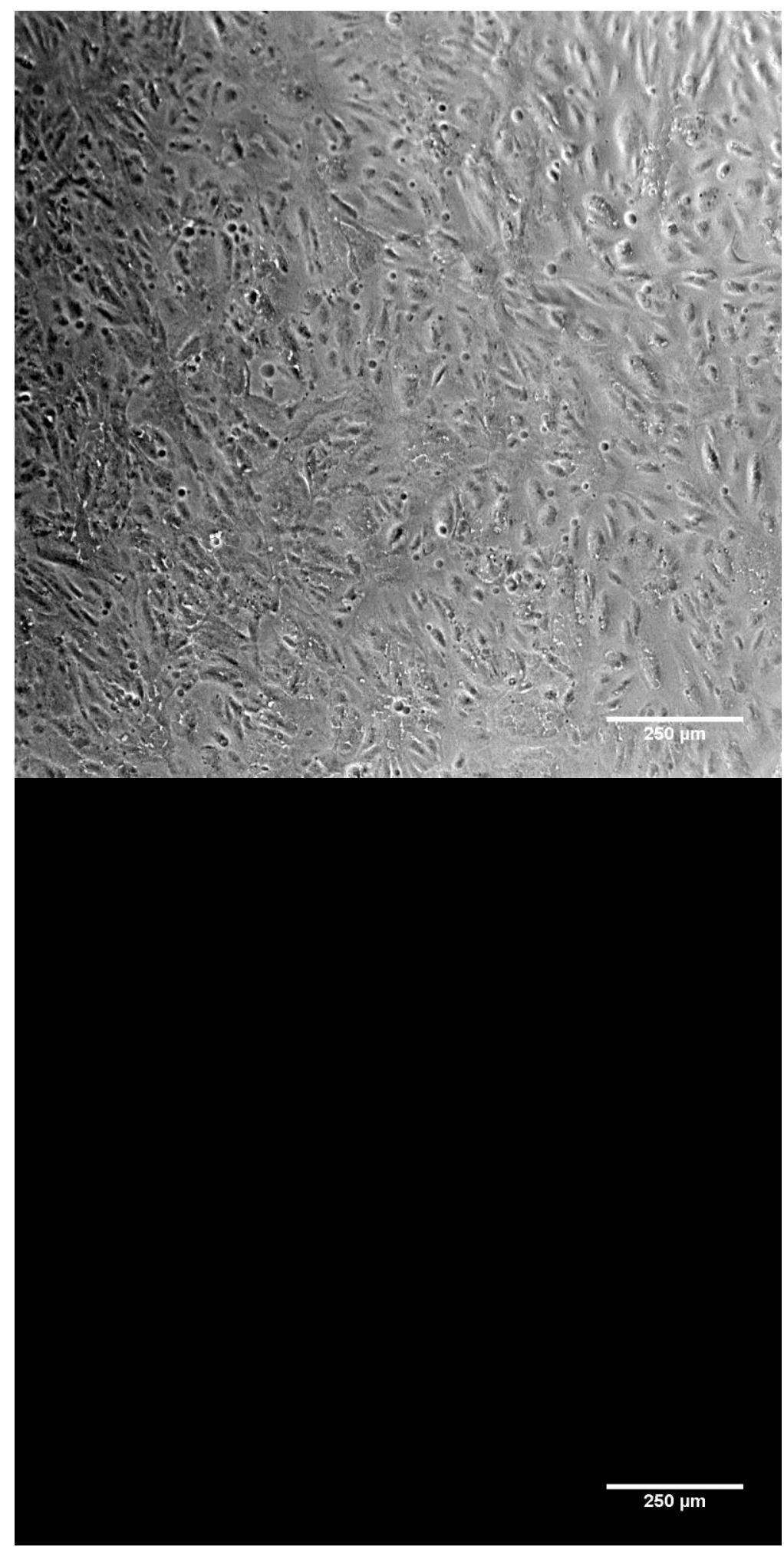

FIGURE A2.12- ARPE 5-1-3 Confluency 


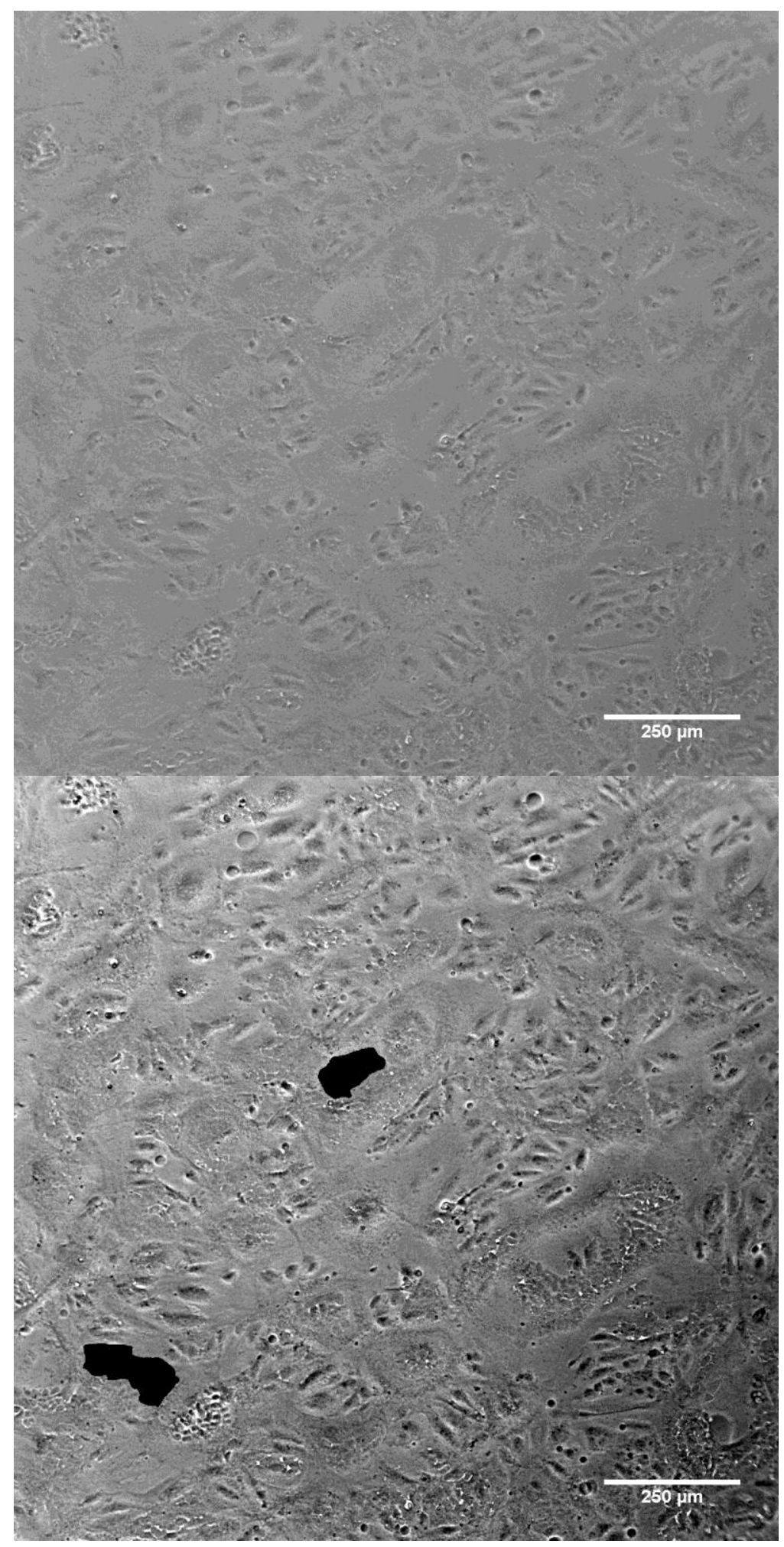

FIGURE A2.13- ARPE 5-2-1 Confluency 


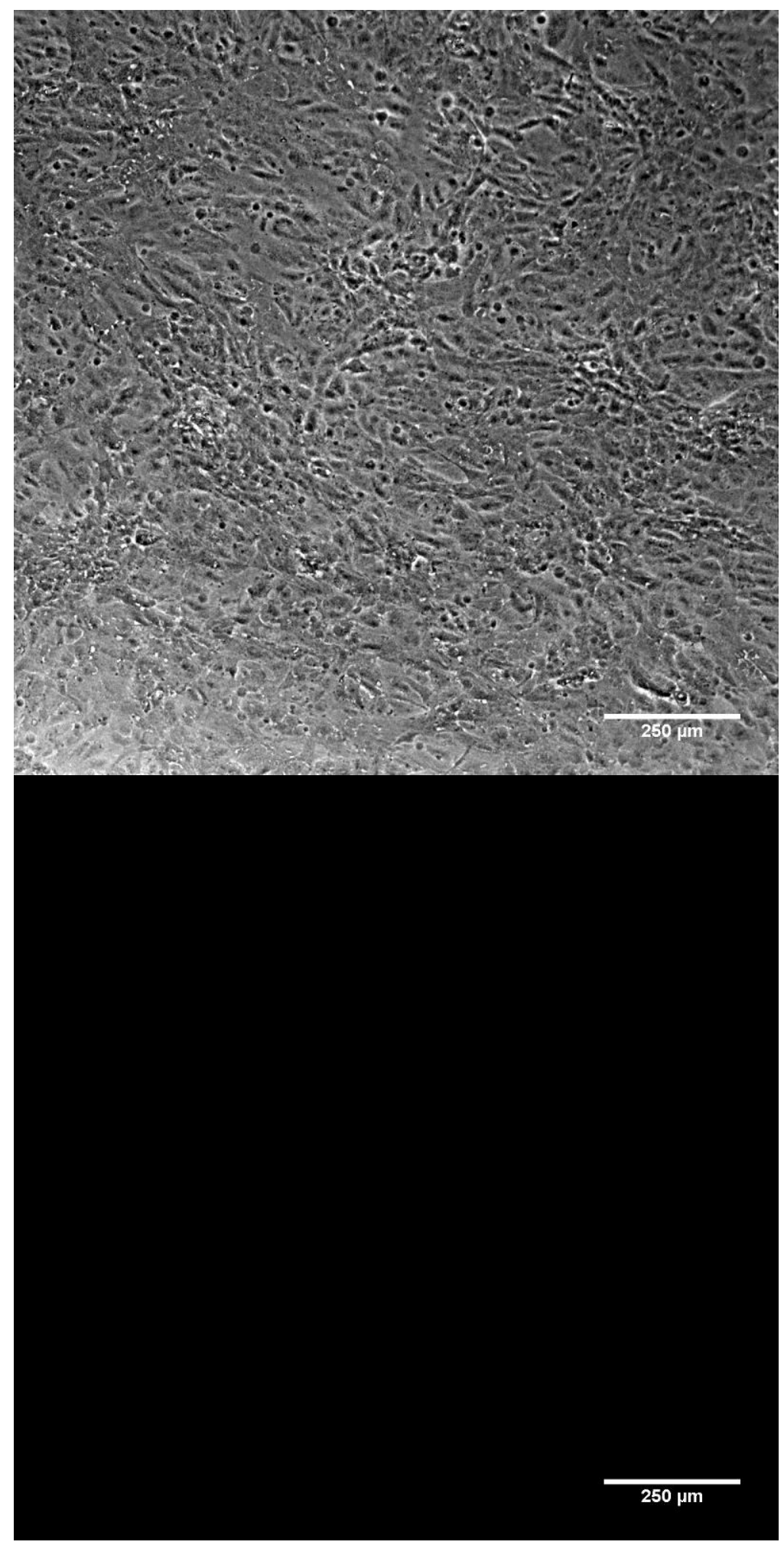

FIGURE A2.14- ARPE 5-2-2 Confluency 


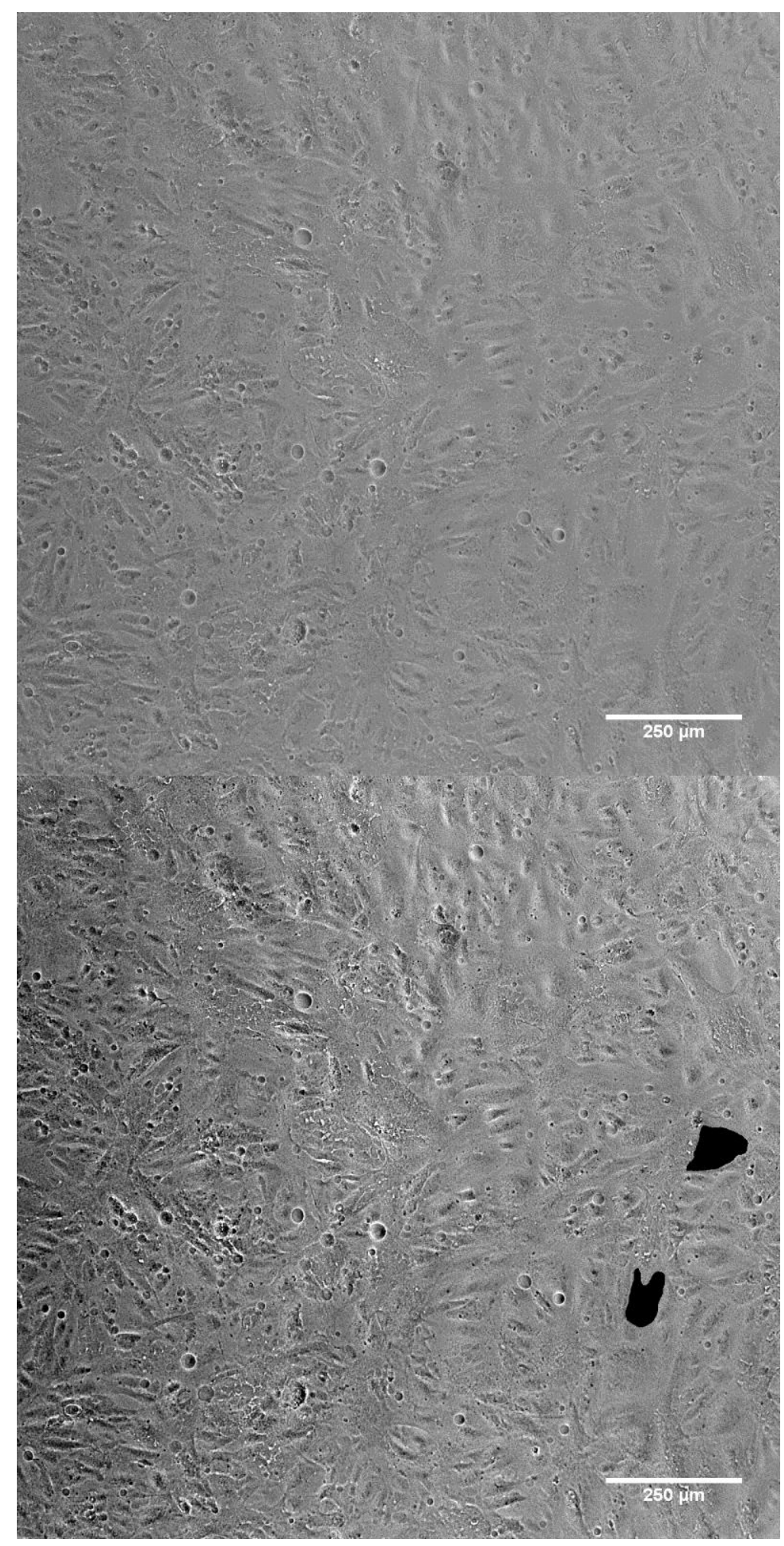

FIGURE A2.15- ARPE 5-2-3 Confluency 


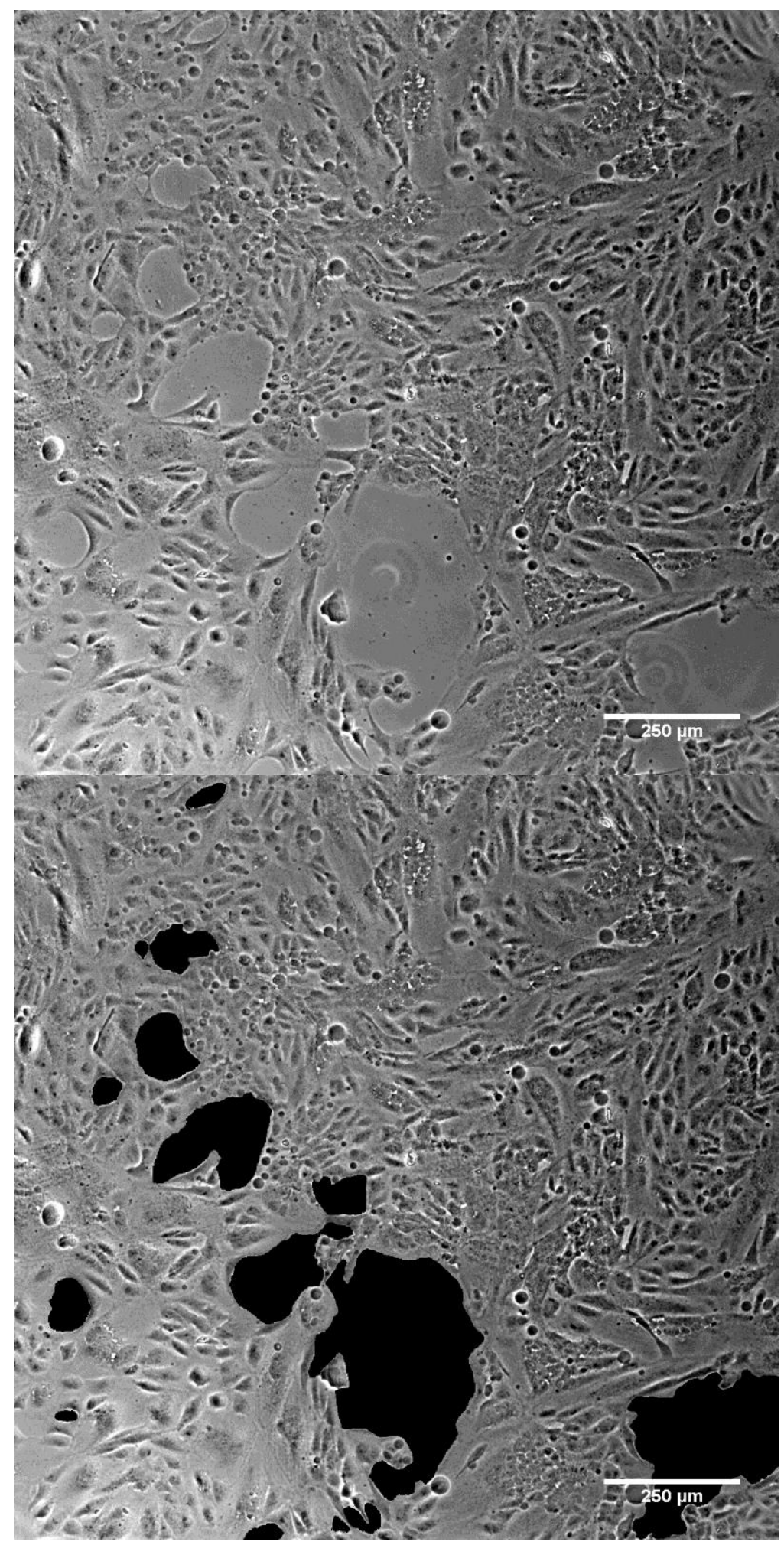

FIGURE A2.16- ARPE 5-3-1 Confluency 


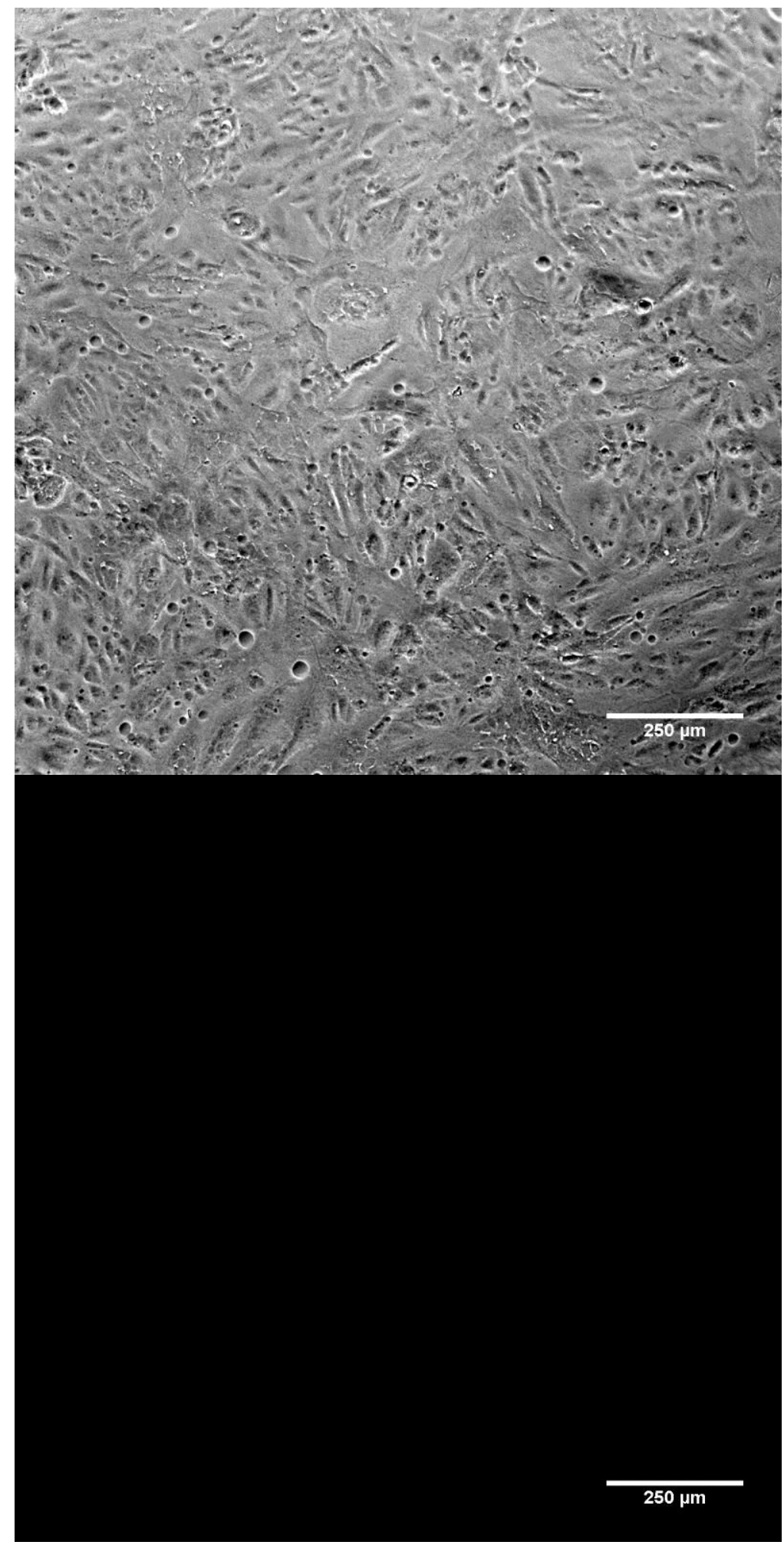

FIGURE A2.17- ARPE 5-3-2 Confluency 


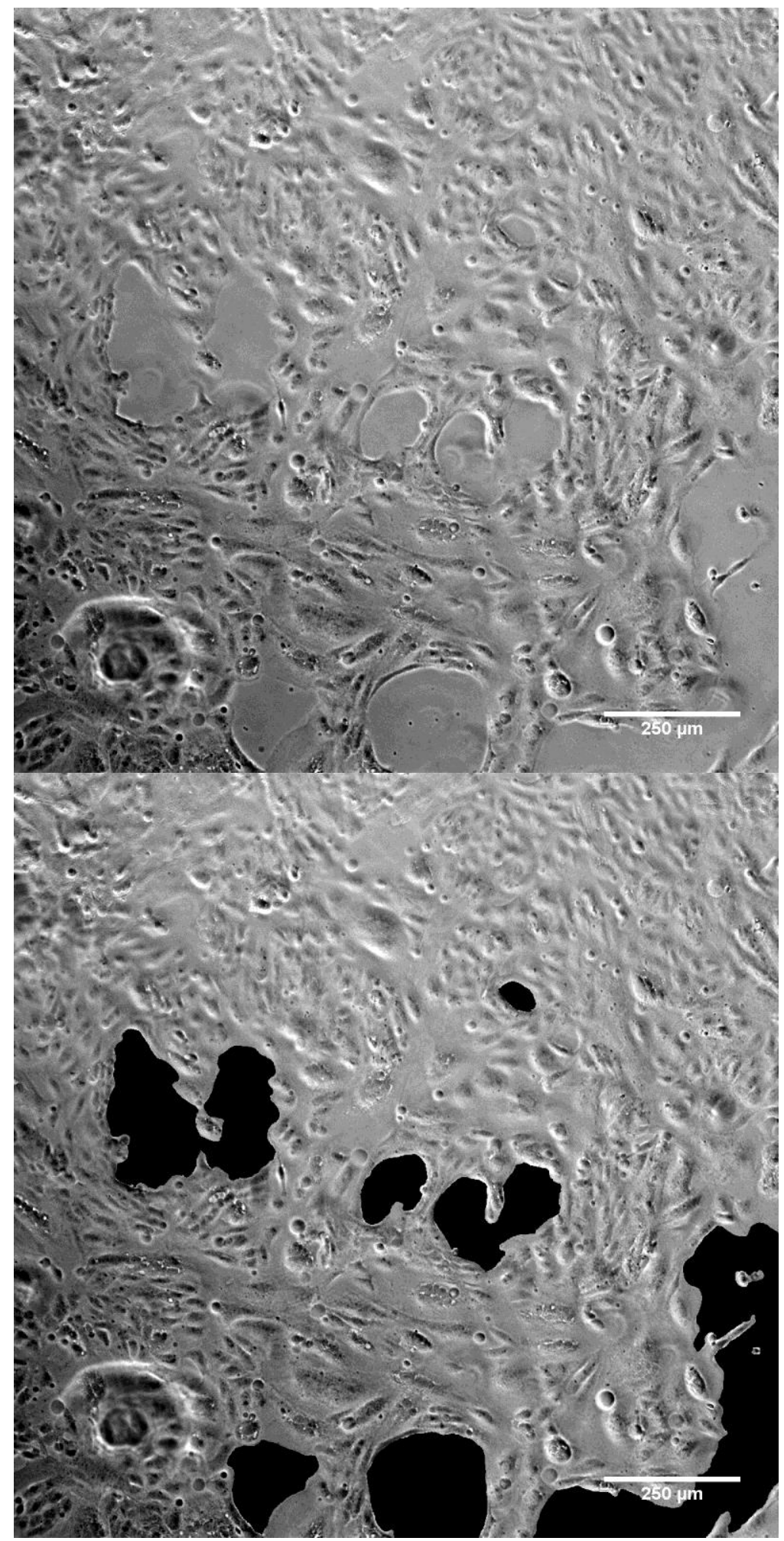

FIGURE A2.18- ARPE 5-3-3 Confluency 


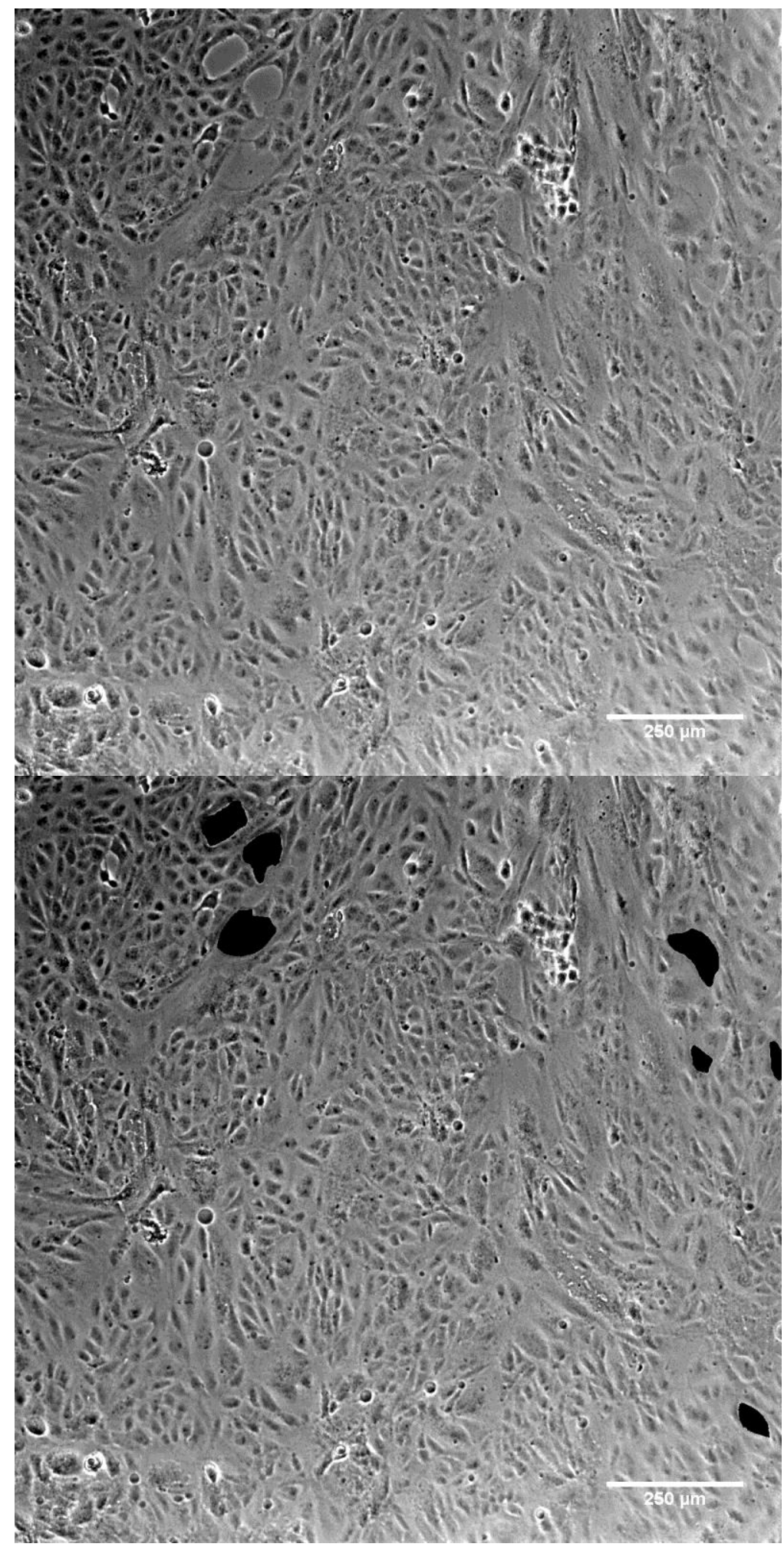

FIGURE A2.19- ARPE 10-1-1 Confluency 


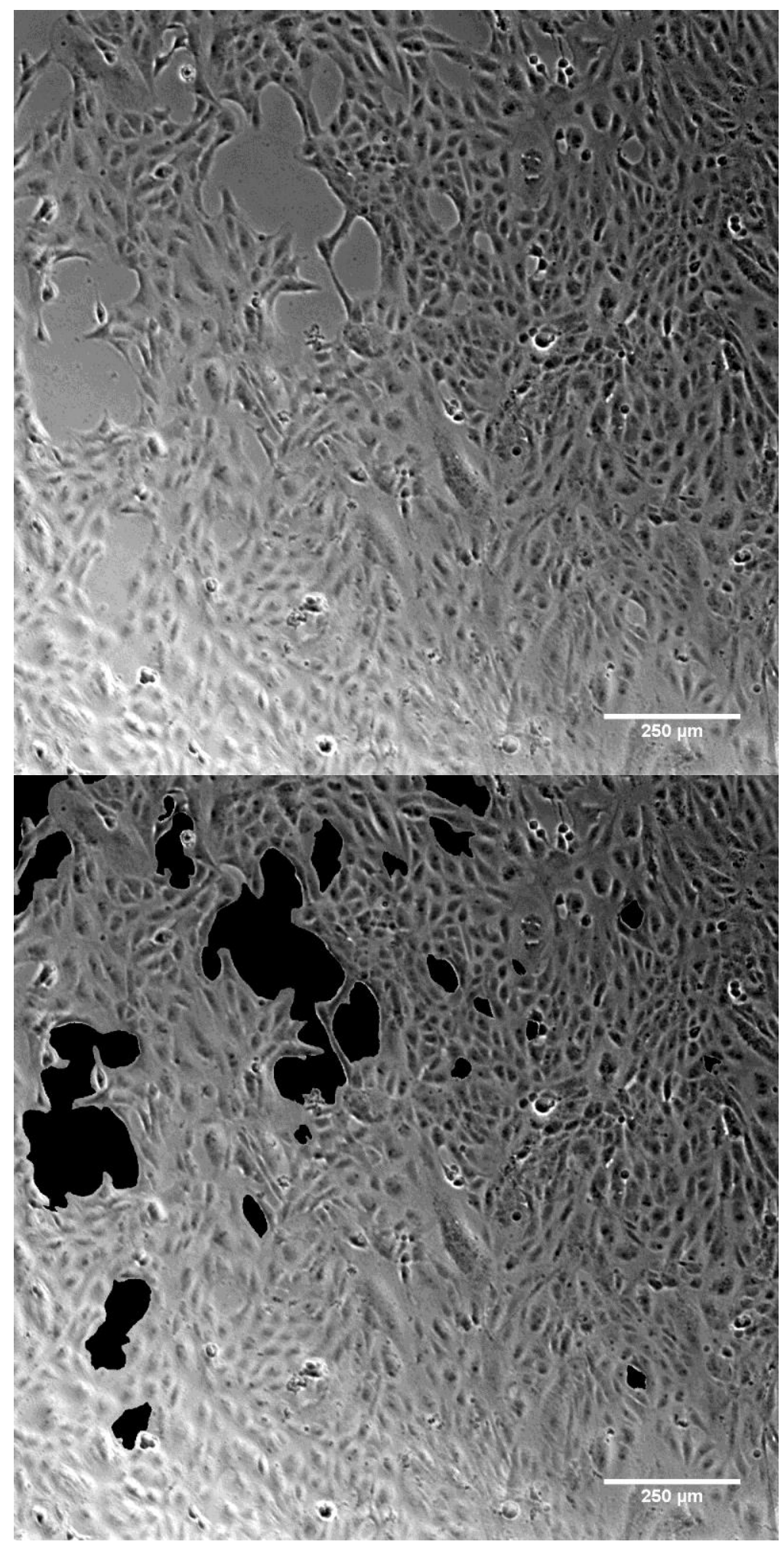

FIGURE A2.20- ARPE 10-1-2 Confluency 


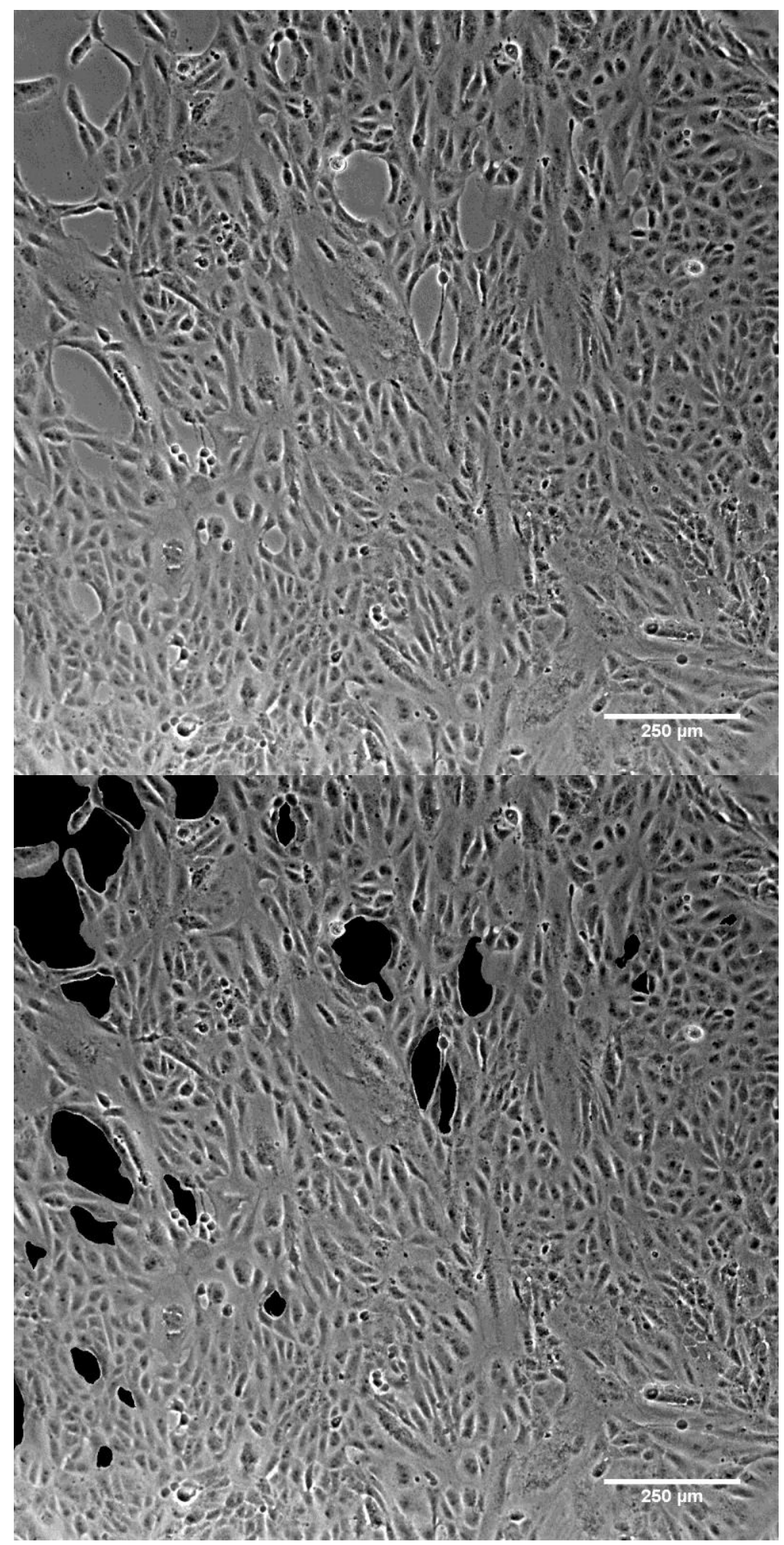

FIGURE A2.21- ARPE 10-1-3 Confluency 


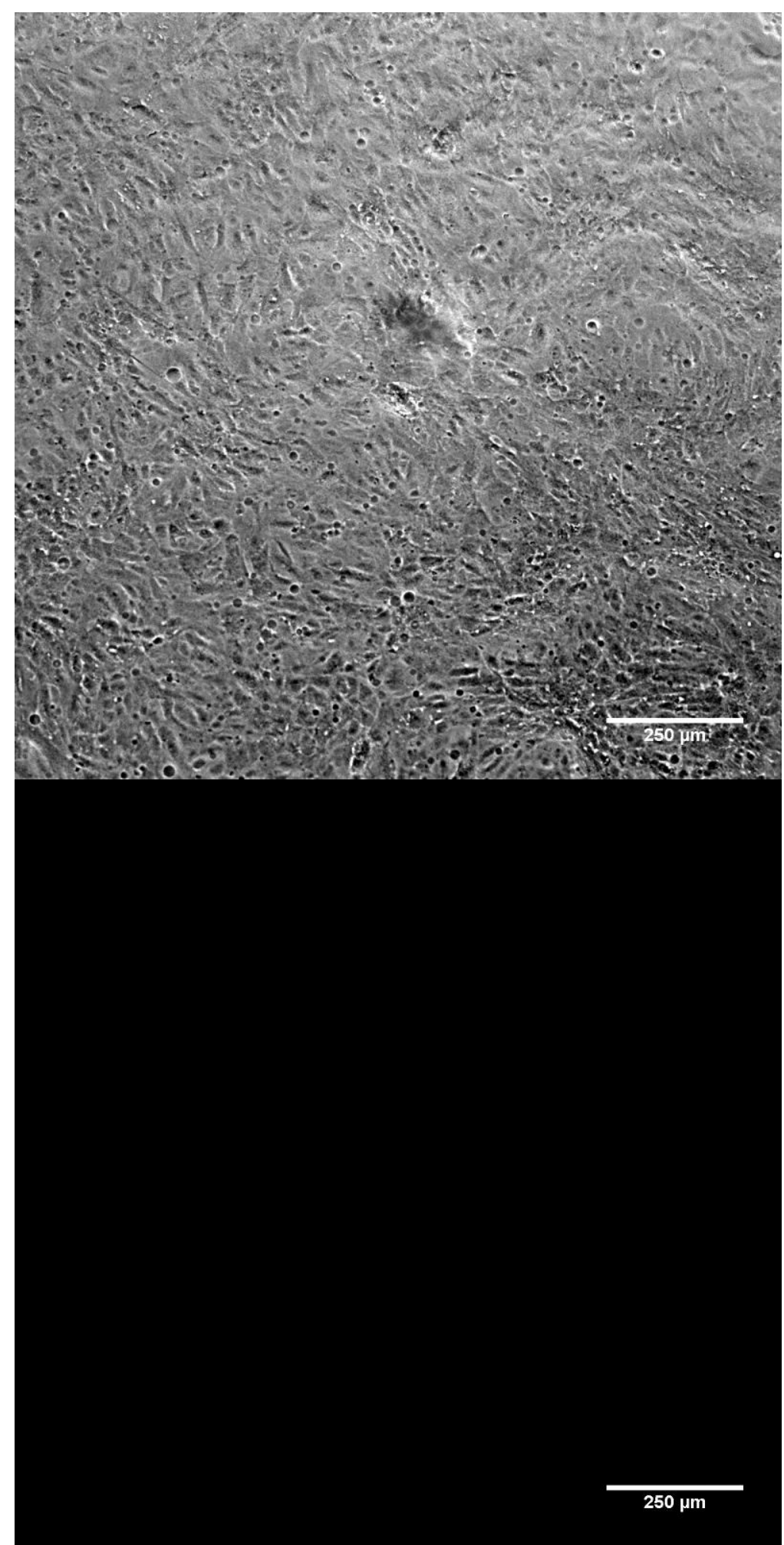

FIGURE A2.22- ARPE 10-2-1 Confluency 


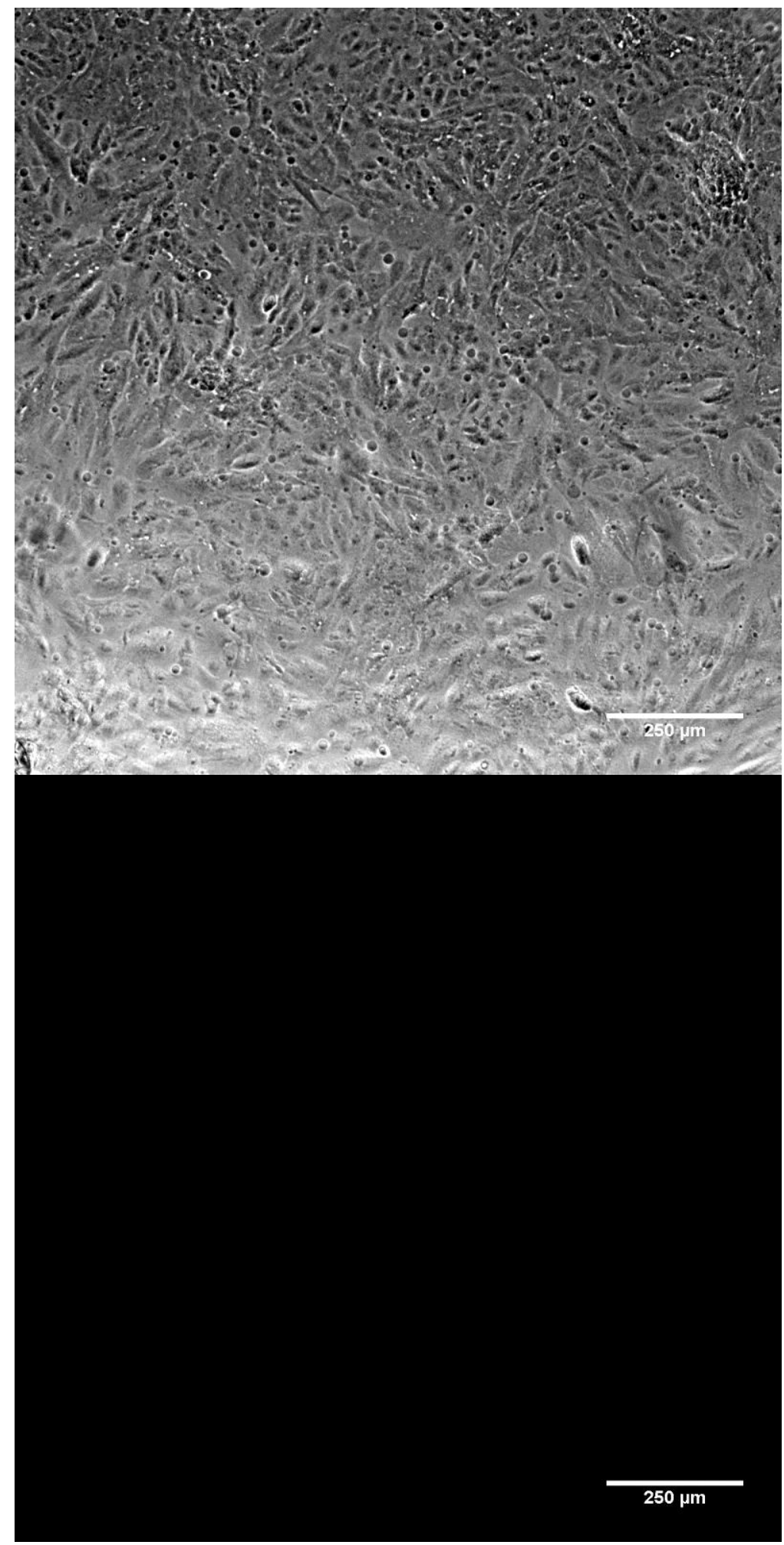

FIGURE A2.23- ARPE 10-2-2 Confluency 


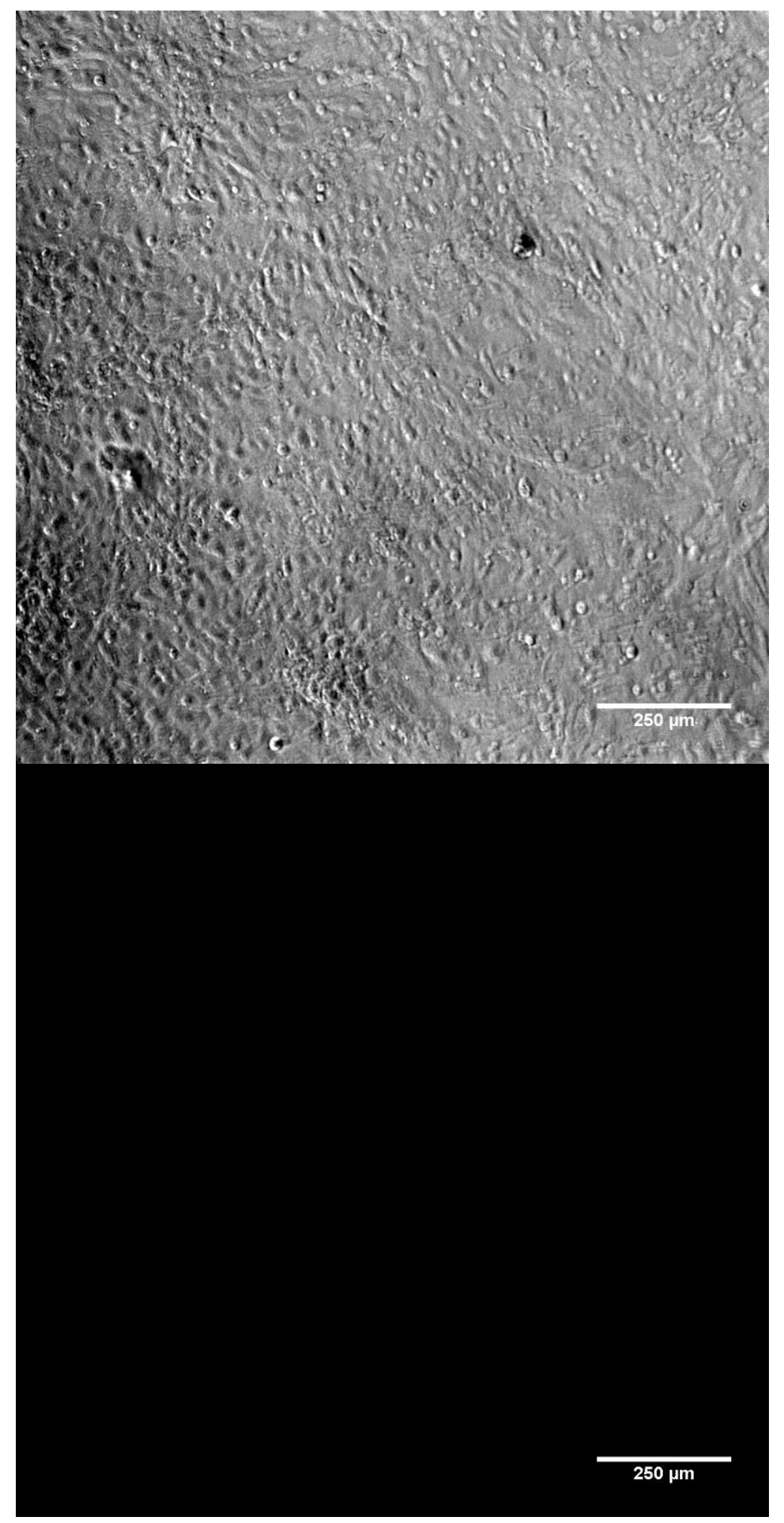

FIGURE A2.24- ARPE 10-2-3 Confluency 


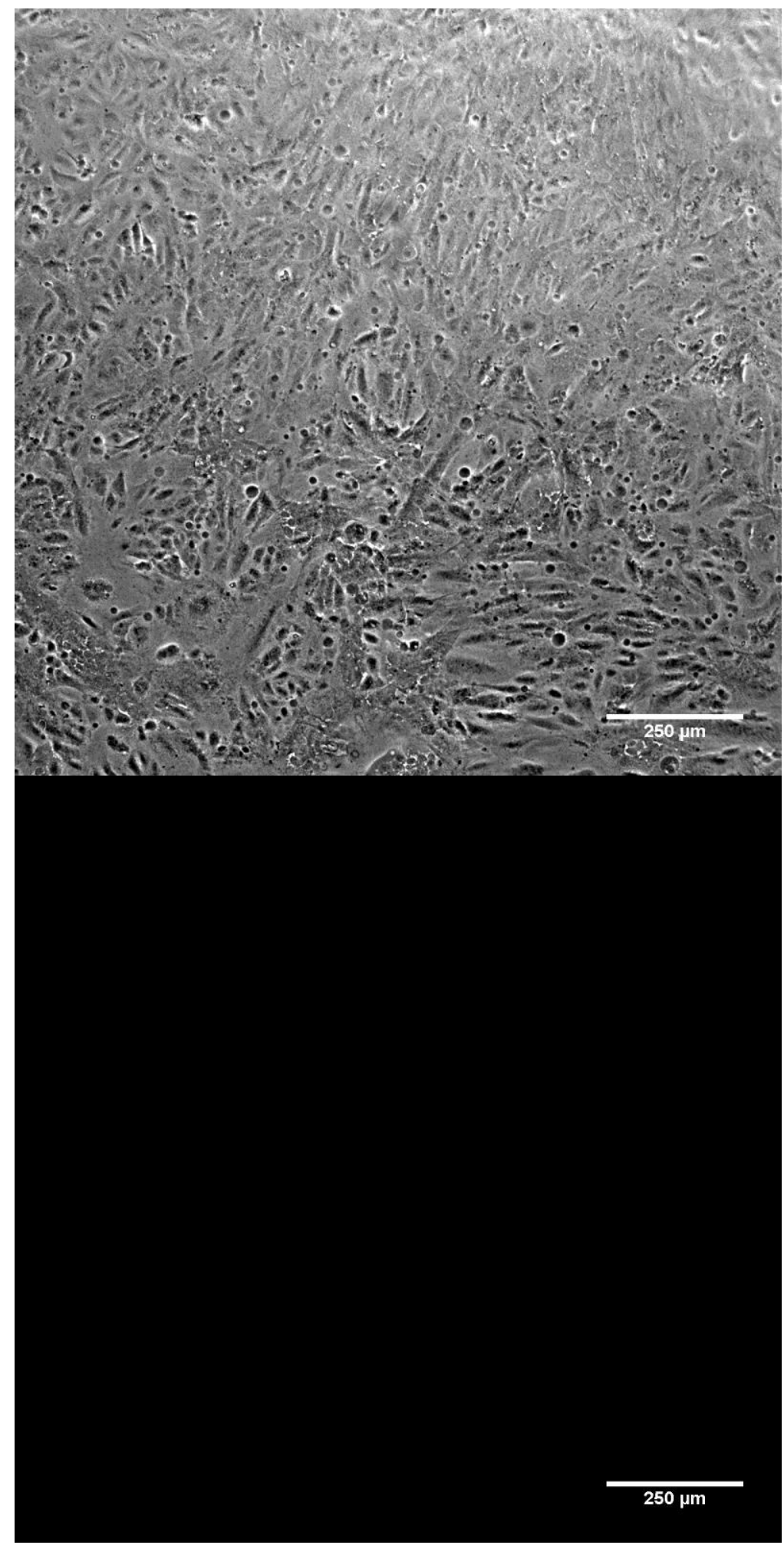

FIGURE A2.25- ARPE 10-3-1 Confluency 


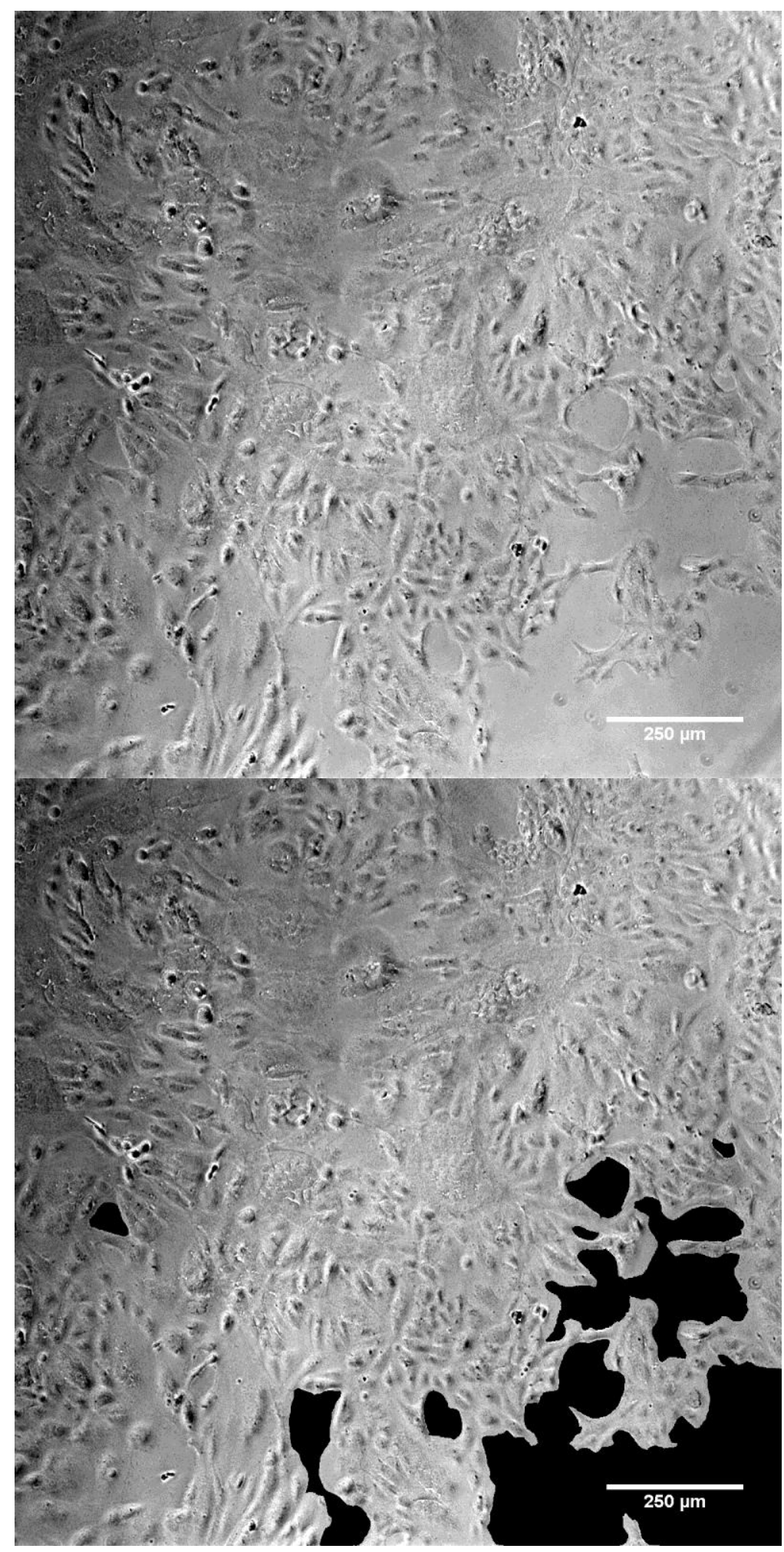

FIGURE A2.26- ARPE 10-3-2 Confluency 


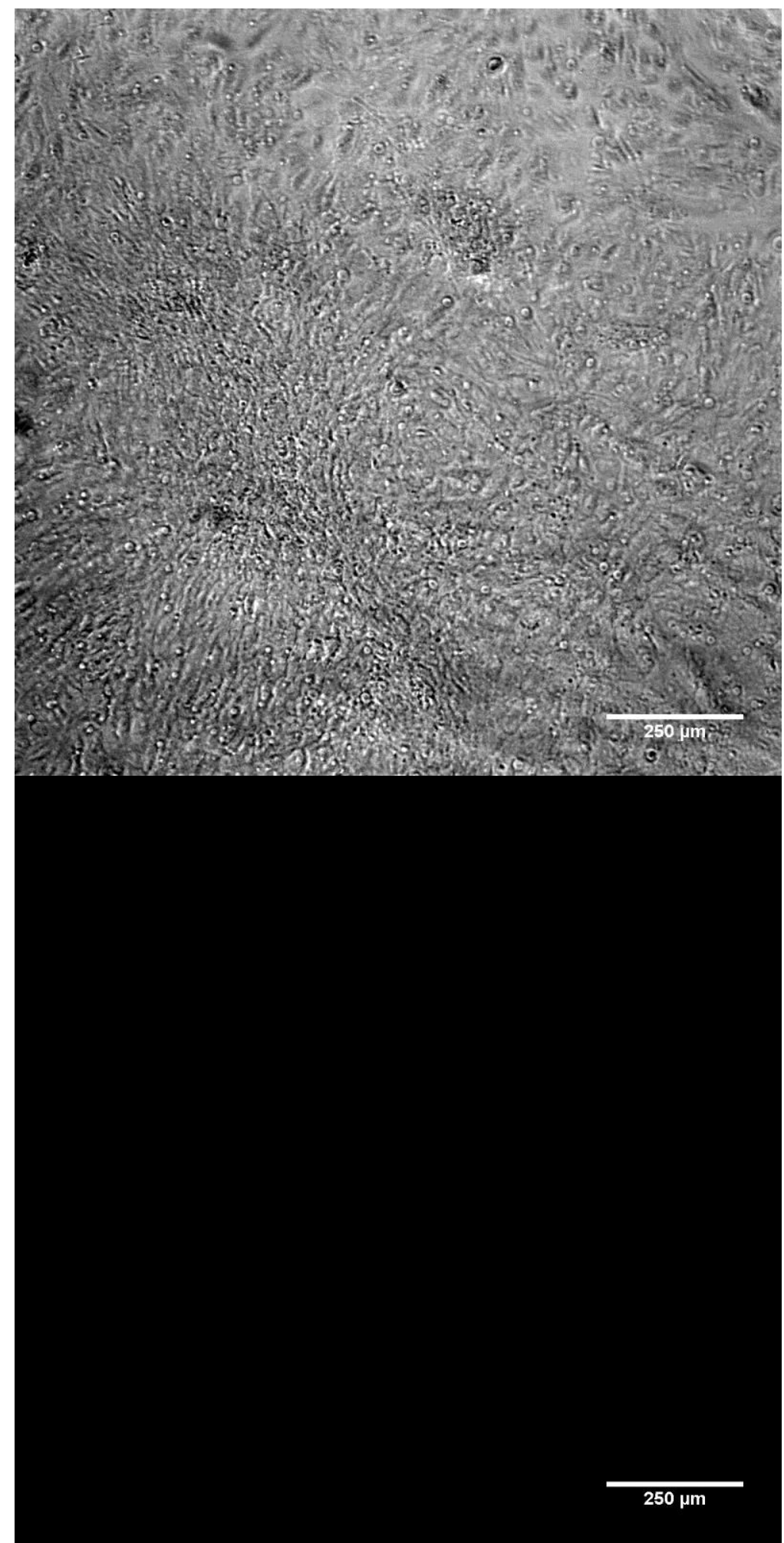

FIGURE A2.27- ARPE 10-3-3 Confluency 


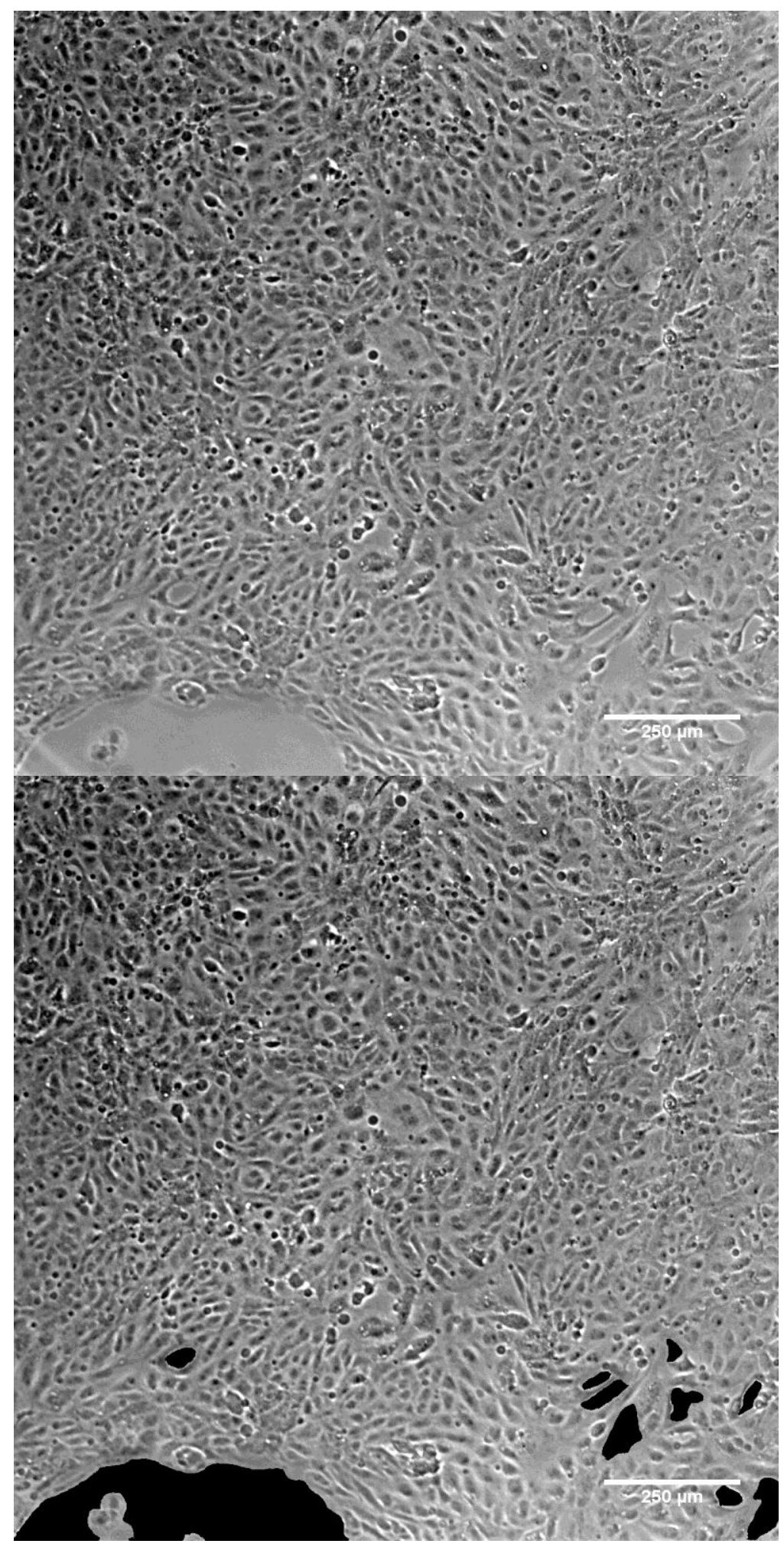

FIGURE A2.28- ARPE 20-1-1 Confluency 


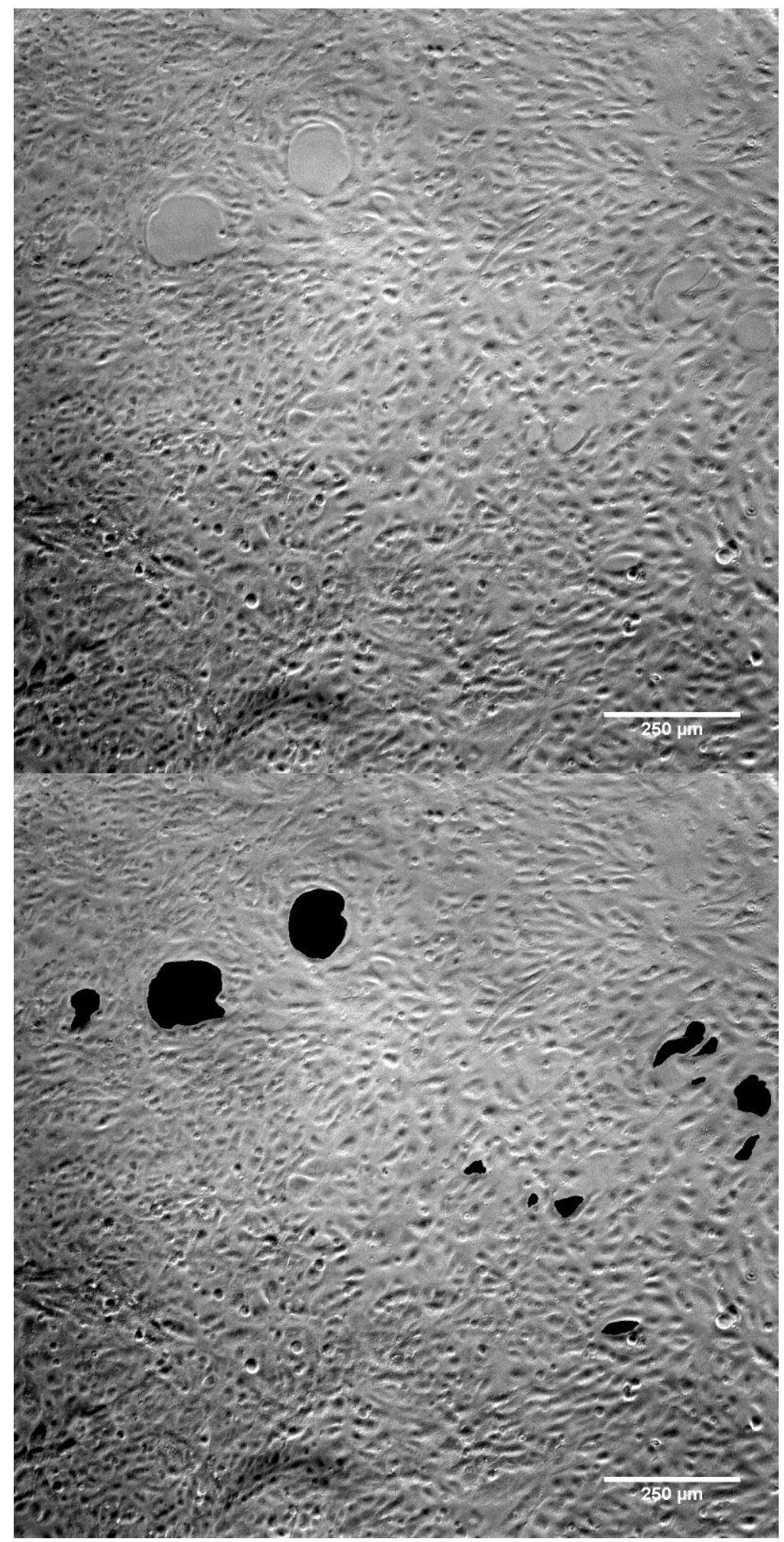

FIGURE A2.29- ARPE 20-1-2 Confluency 


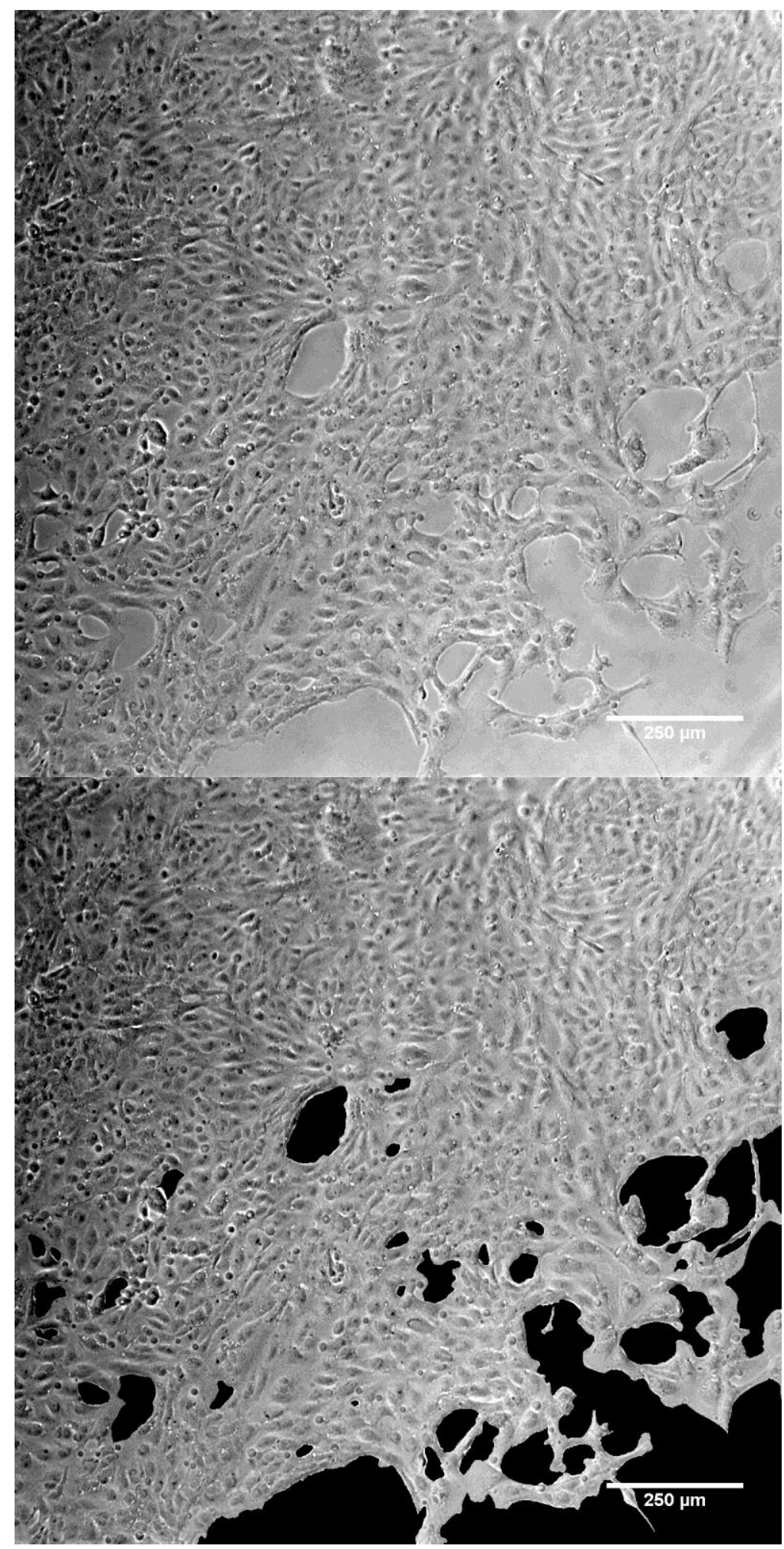

FIGURE A2.30- ARPE 20-1-3 Confluency 


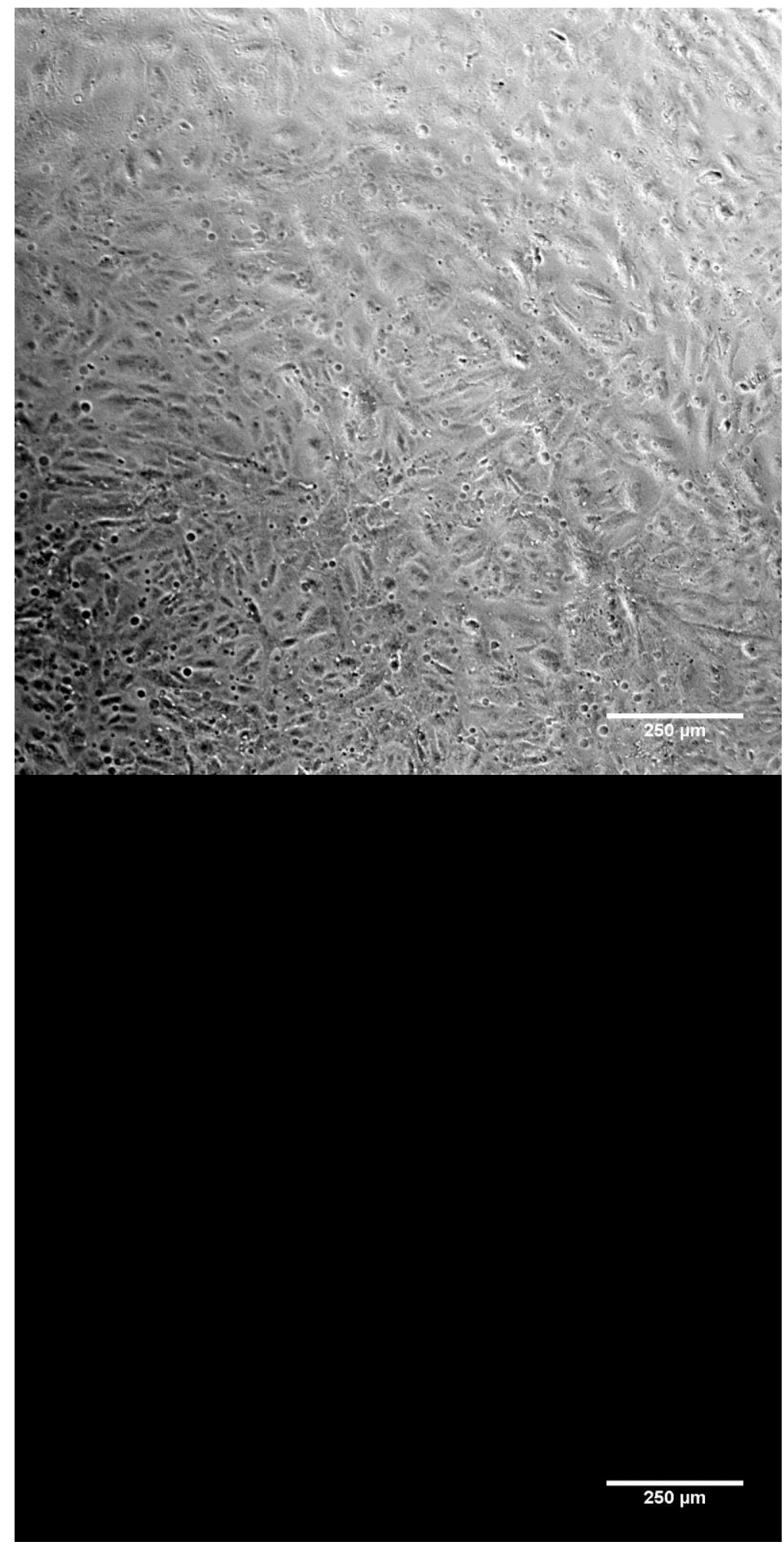

FIGURE A2.31- ARPE 20-2-1 Confluency 


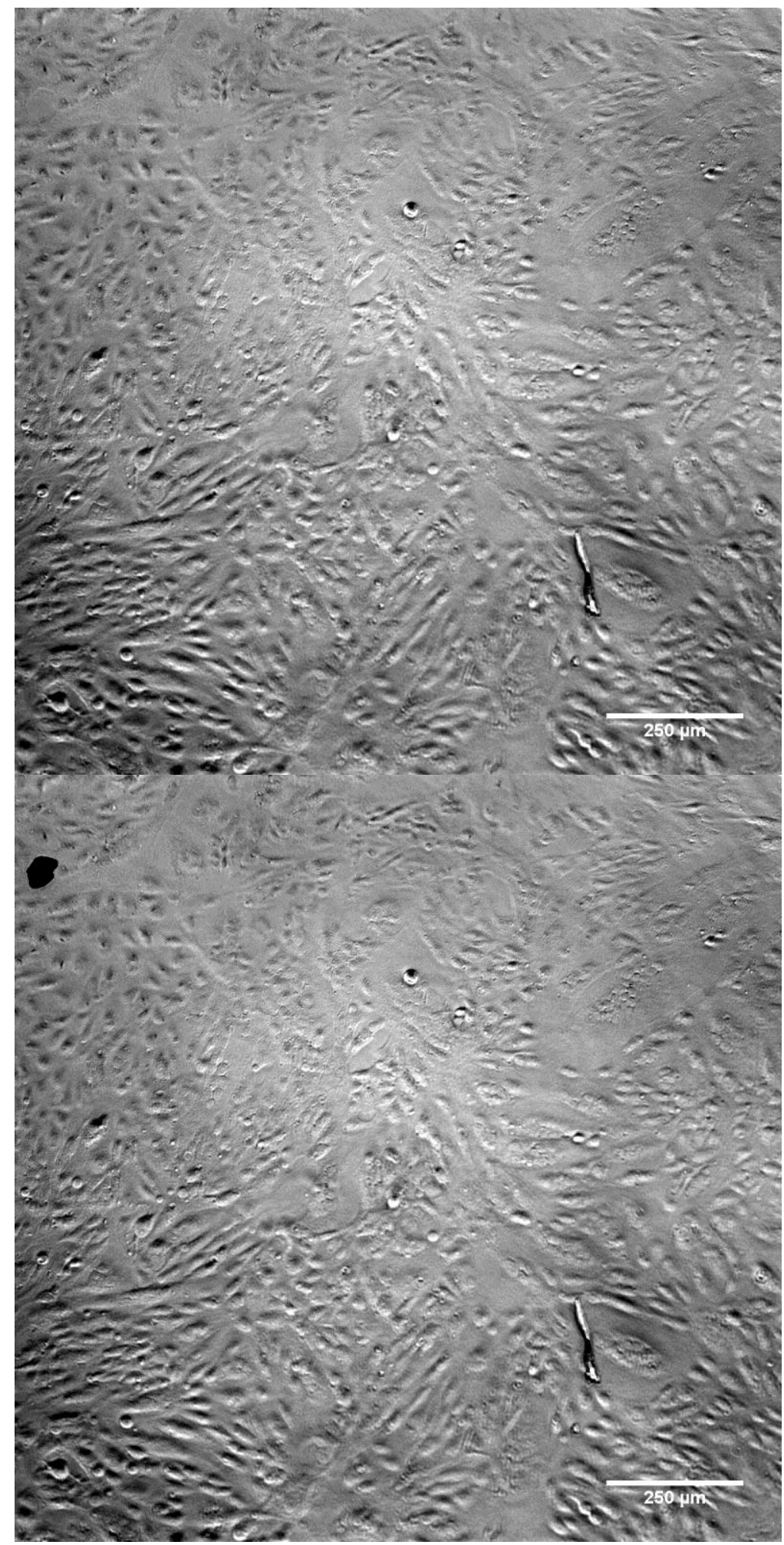

FIGURE A2.32- ARPE 20-2-2 Confluency 


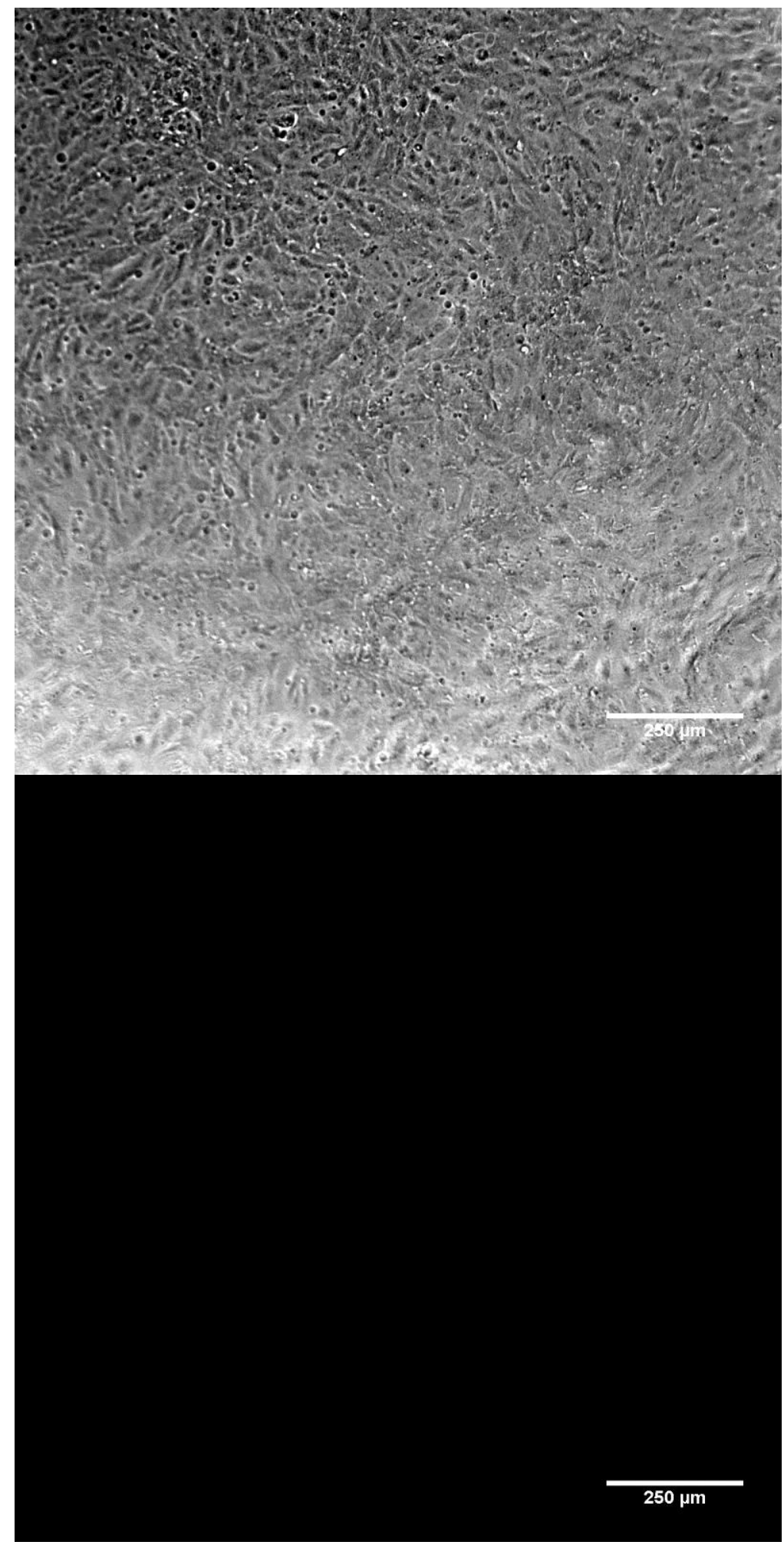

FIGURE A2.33- ARPE 20-2-3 Confluency 


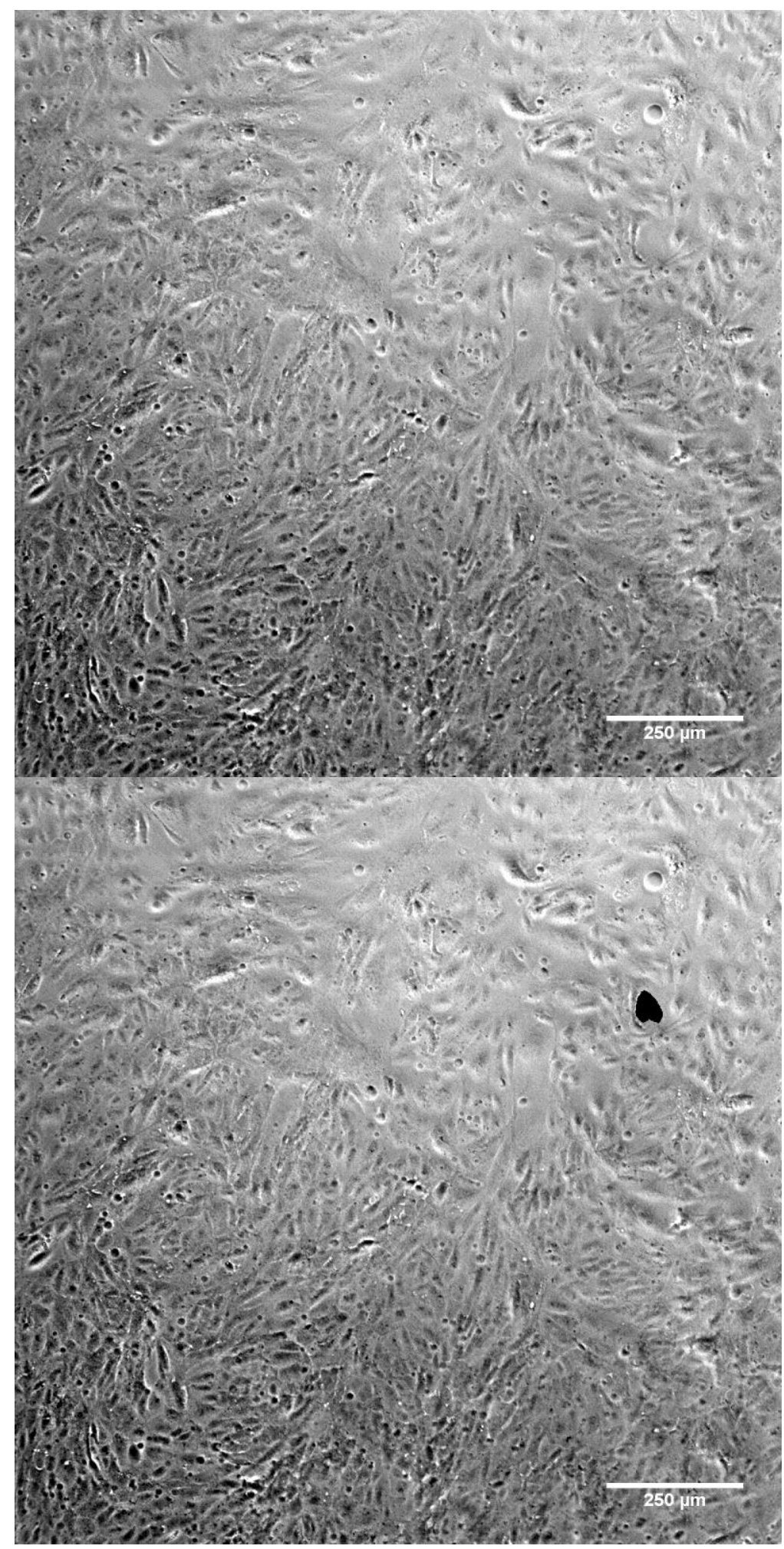

FIGURE A2.34- ARPE 20-3-1 Confluency 


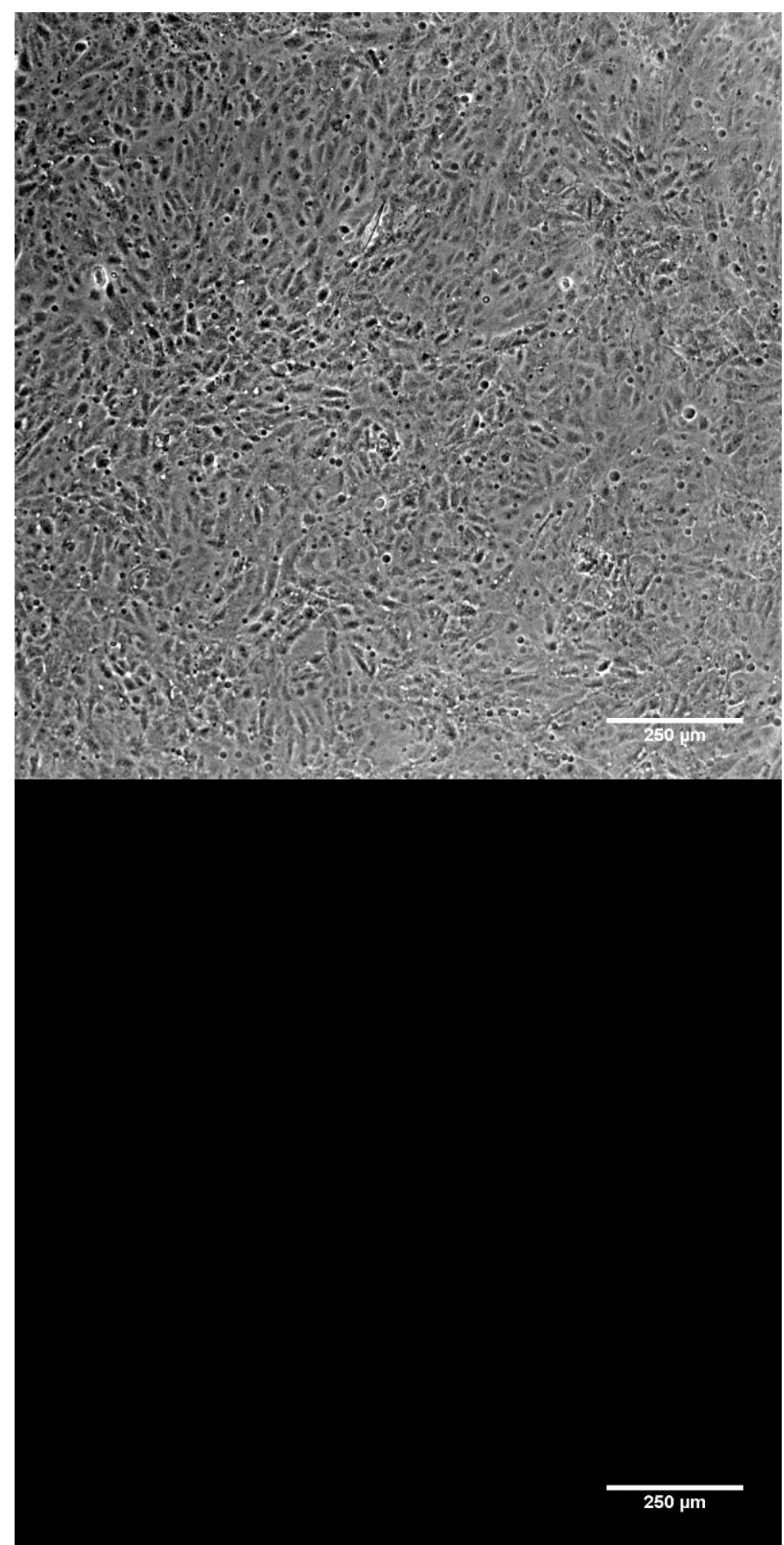

FIGURE A2.35- ARPE 20-3-2 Confluency 


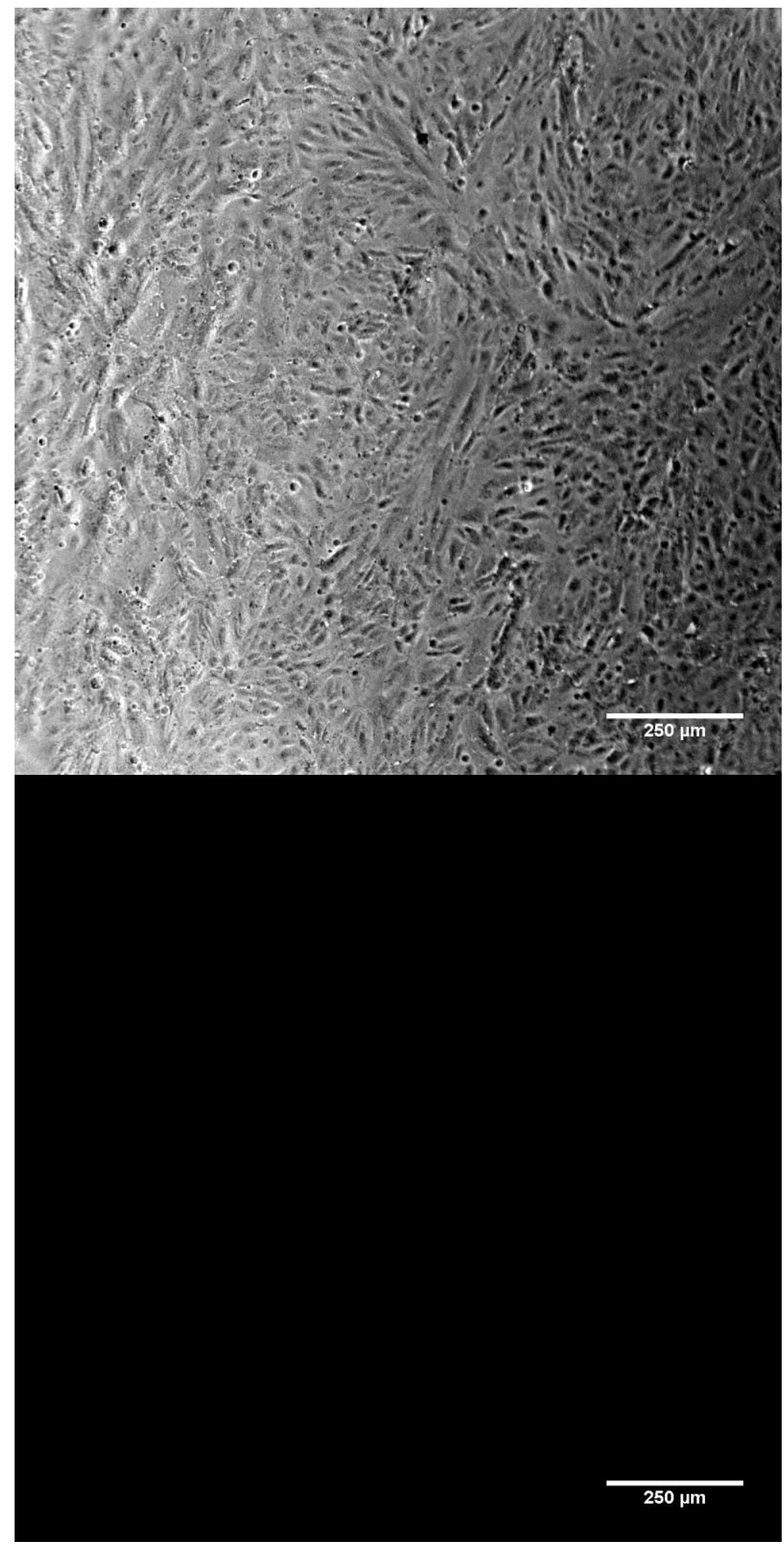

FIGURE A2.36- ARPE 20-3-3 Confluency 


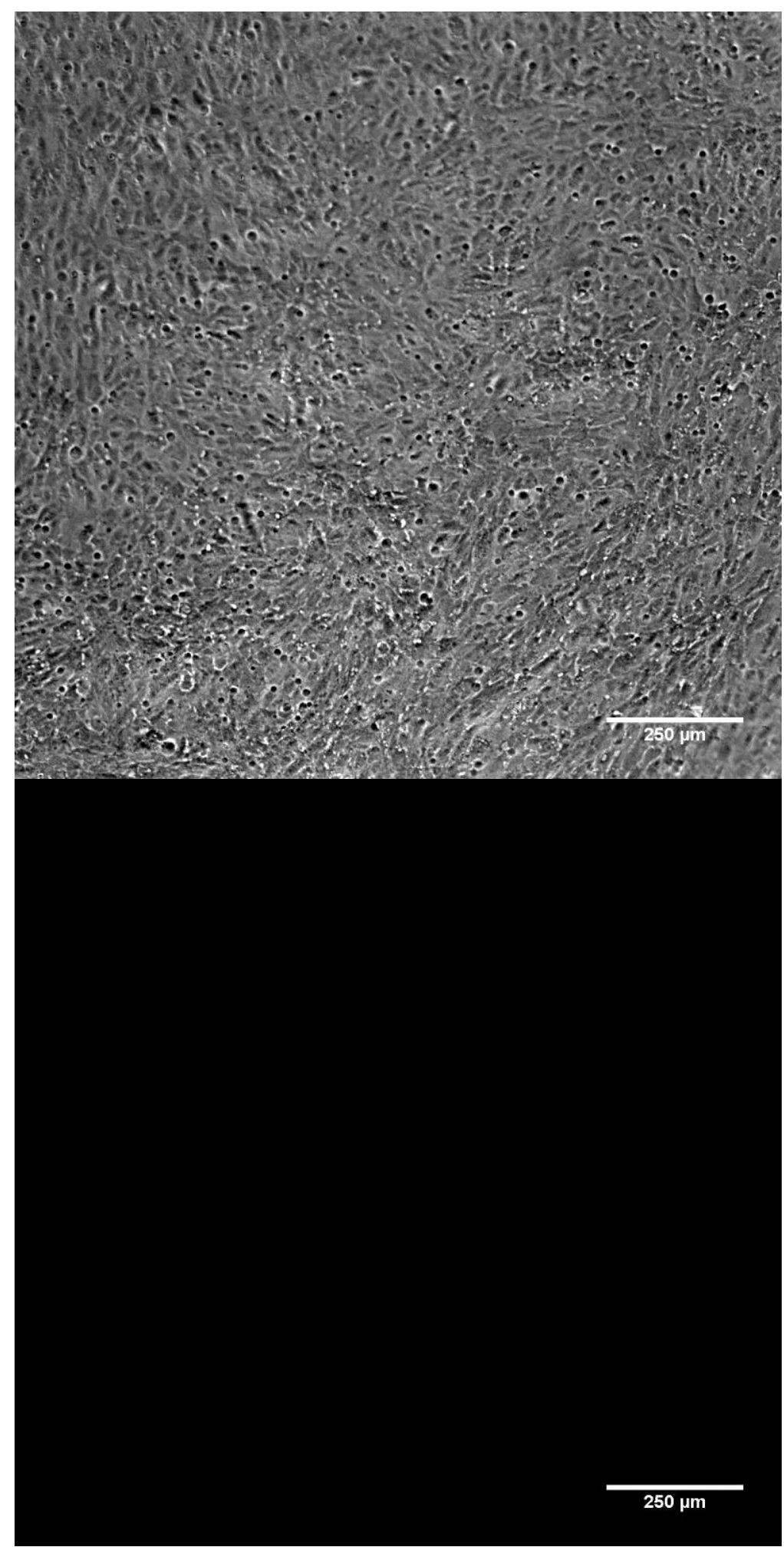

FIGURE A2.37- ARPE FN-1-1 Confluency 


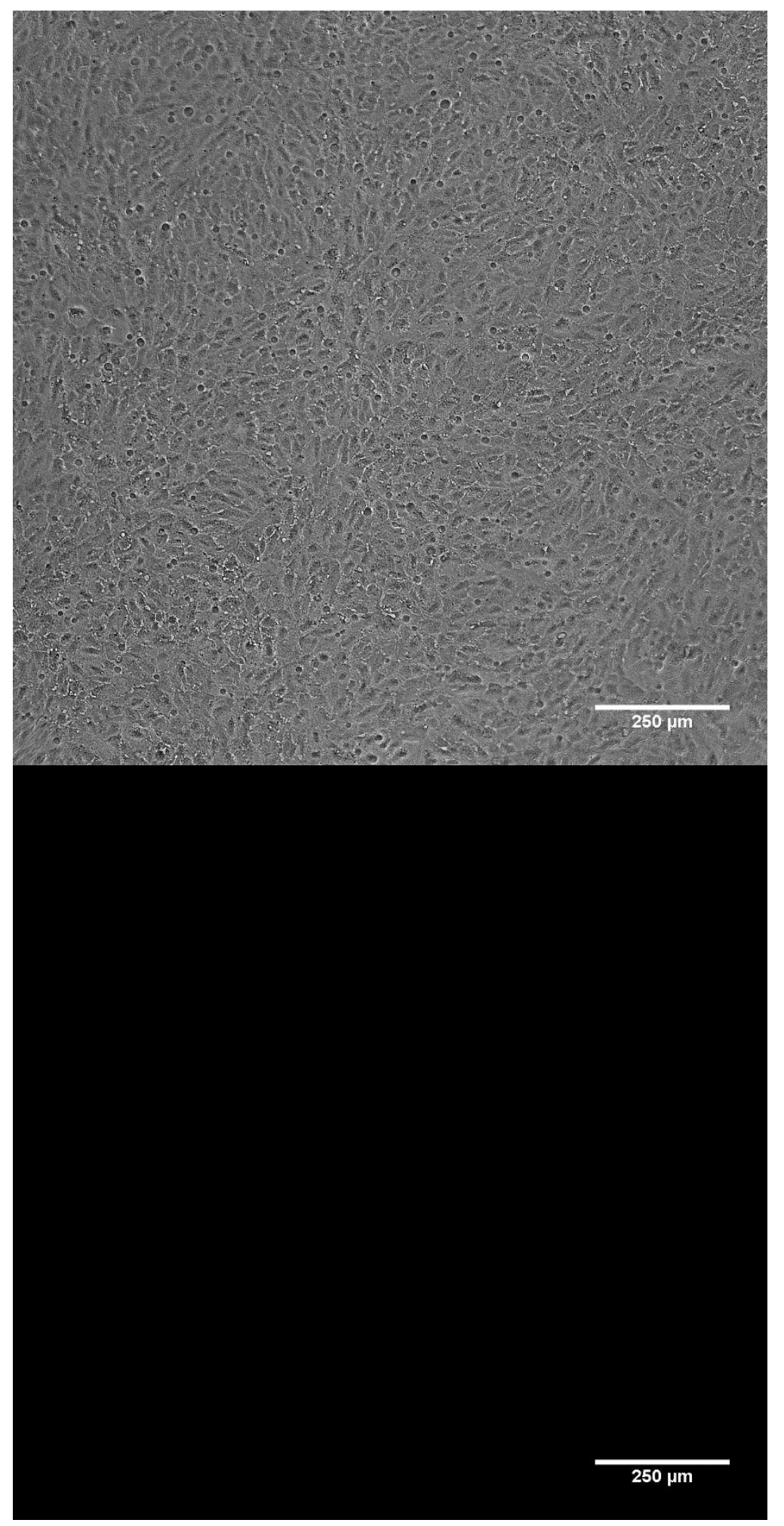

FIGURE A2.38- ARPE FN-1-2 Confluency 


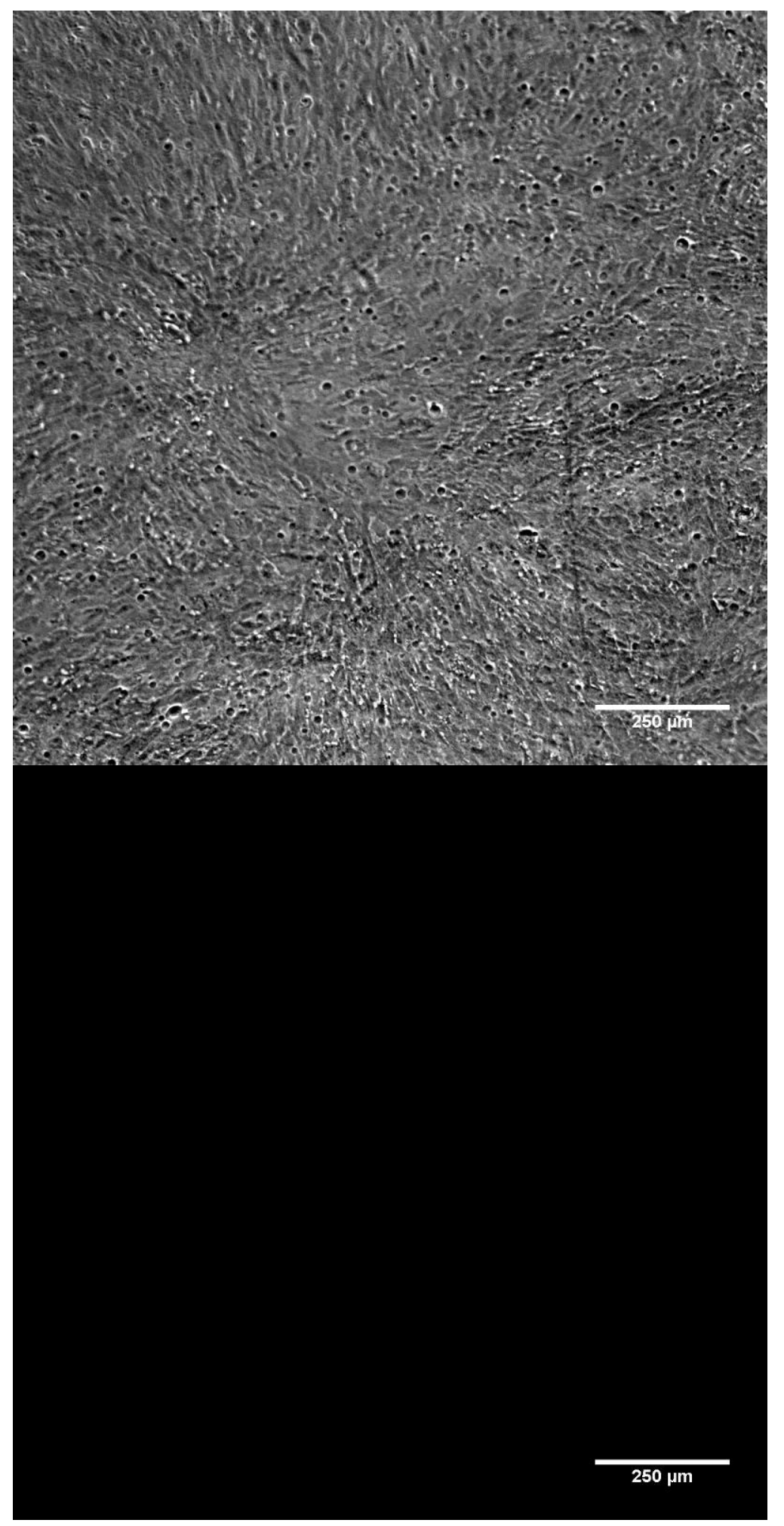

FIGURE A2.39- ARPE FN-1-3 Confluency 


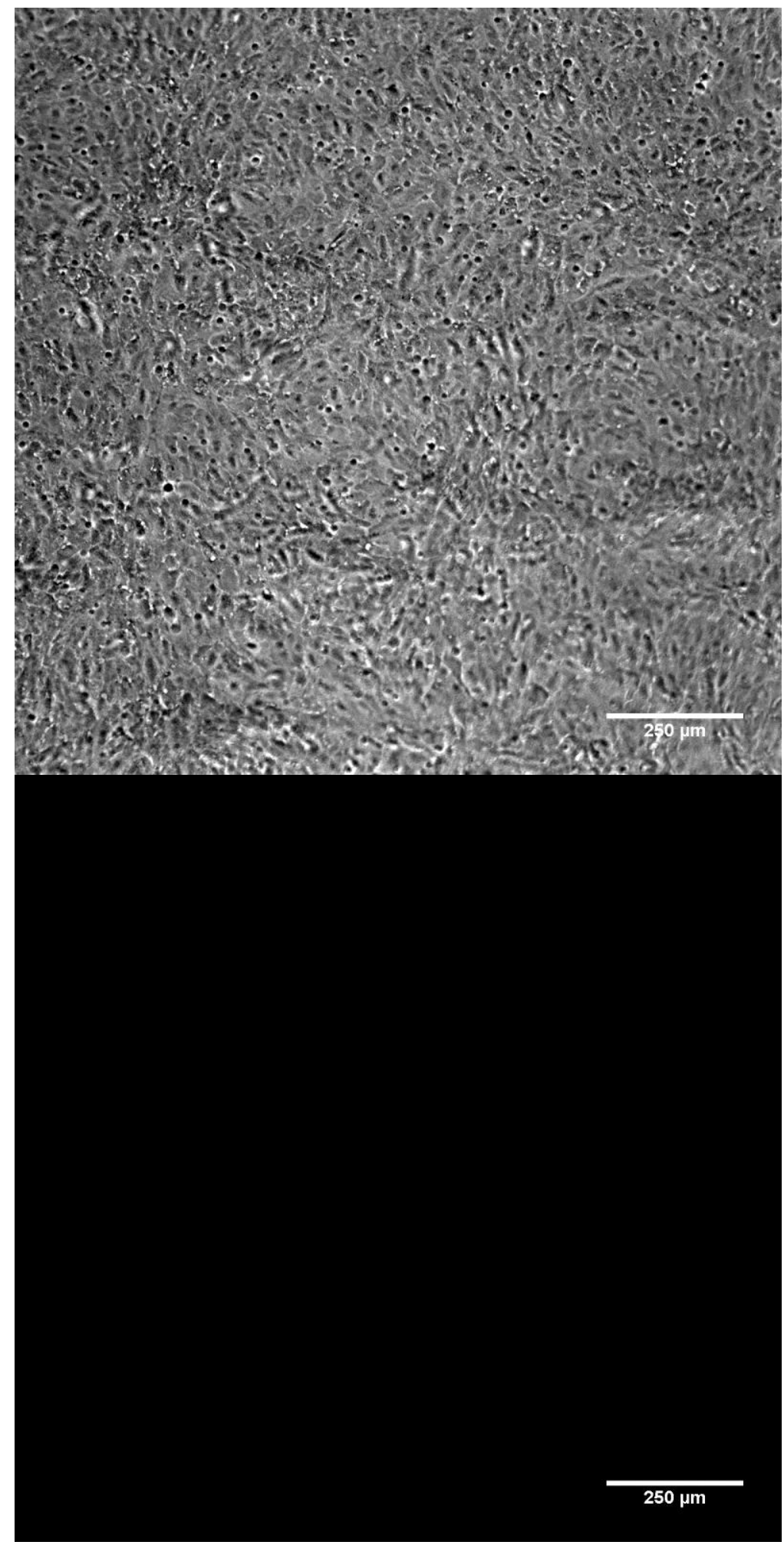

FIGURE A2.40- ARPE FN-2-1 Confluency 


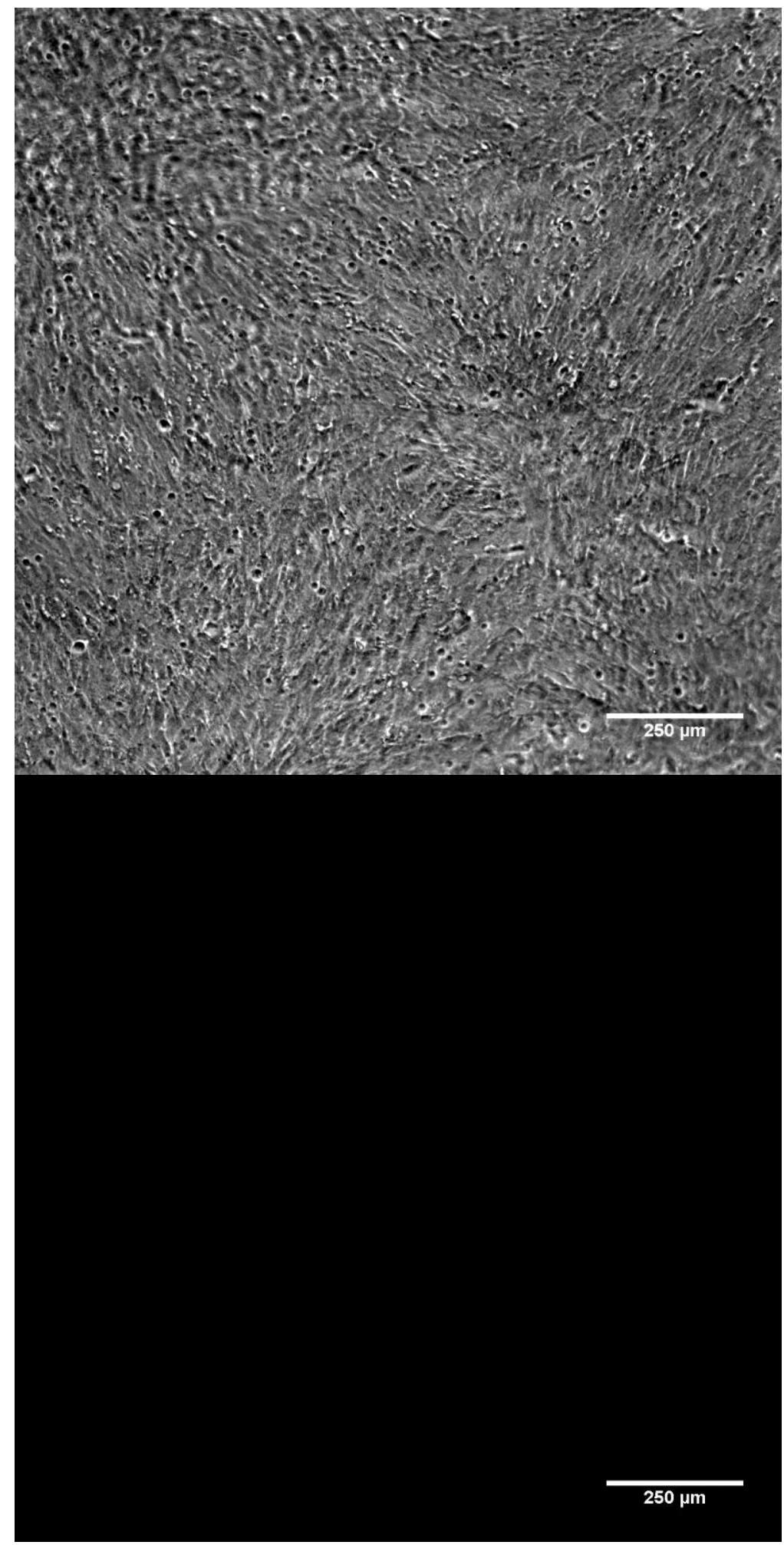

FIGURE A2.41- ARPE FN-2-2 Confluency 


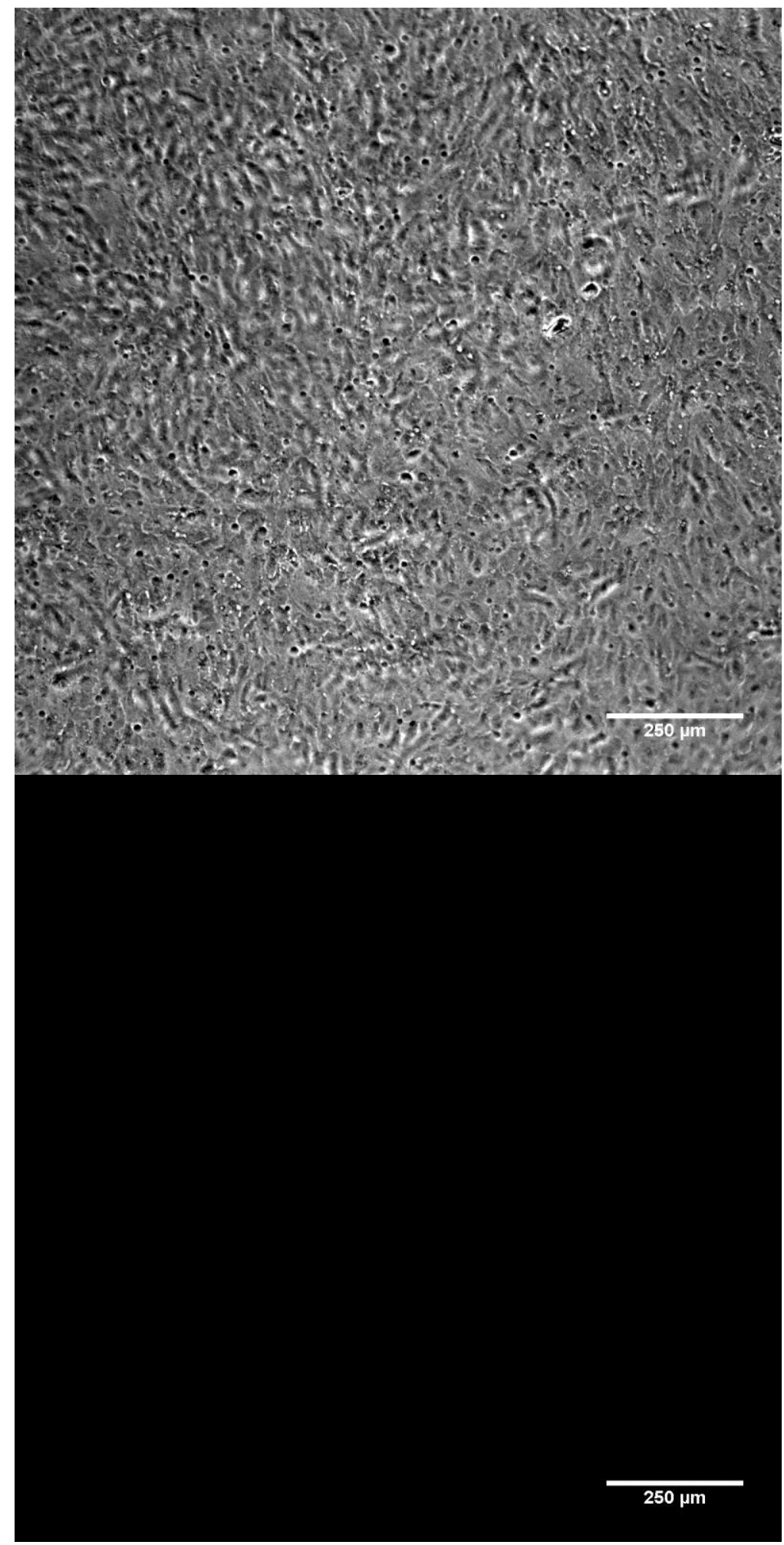

FIGURE A2.42- ARPE FN-2-3 Confluency 


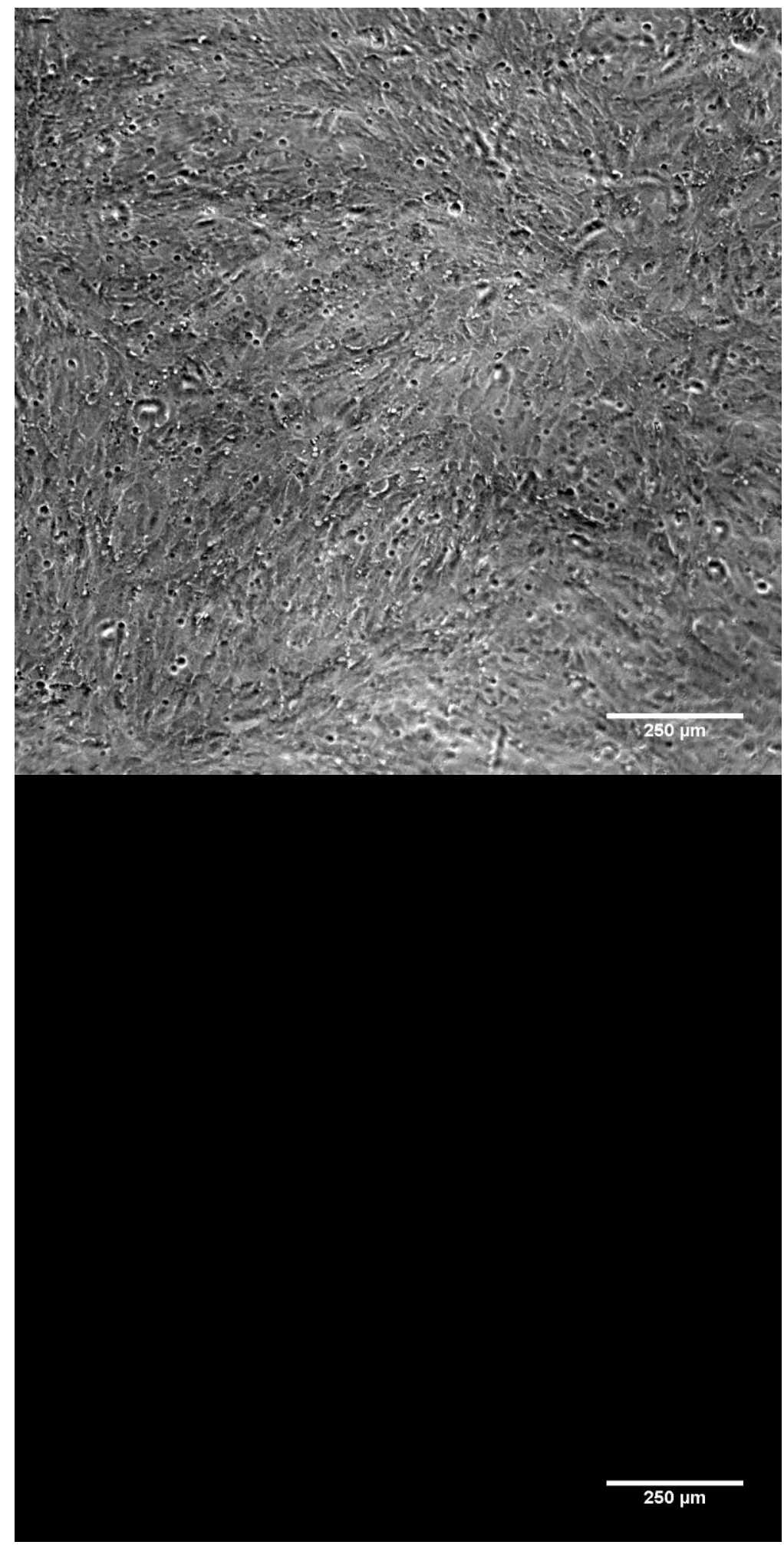

FIGURE A2.43- ARPE FN-3-1 Confluency 


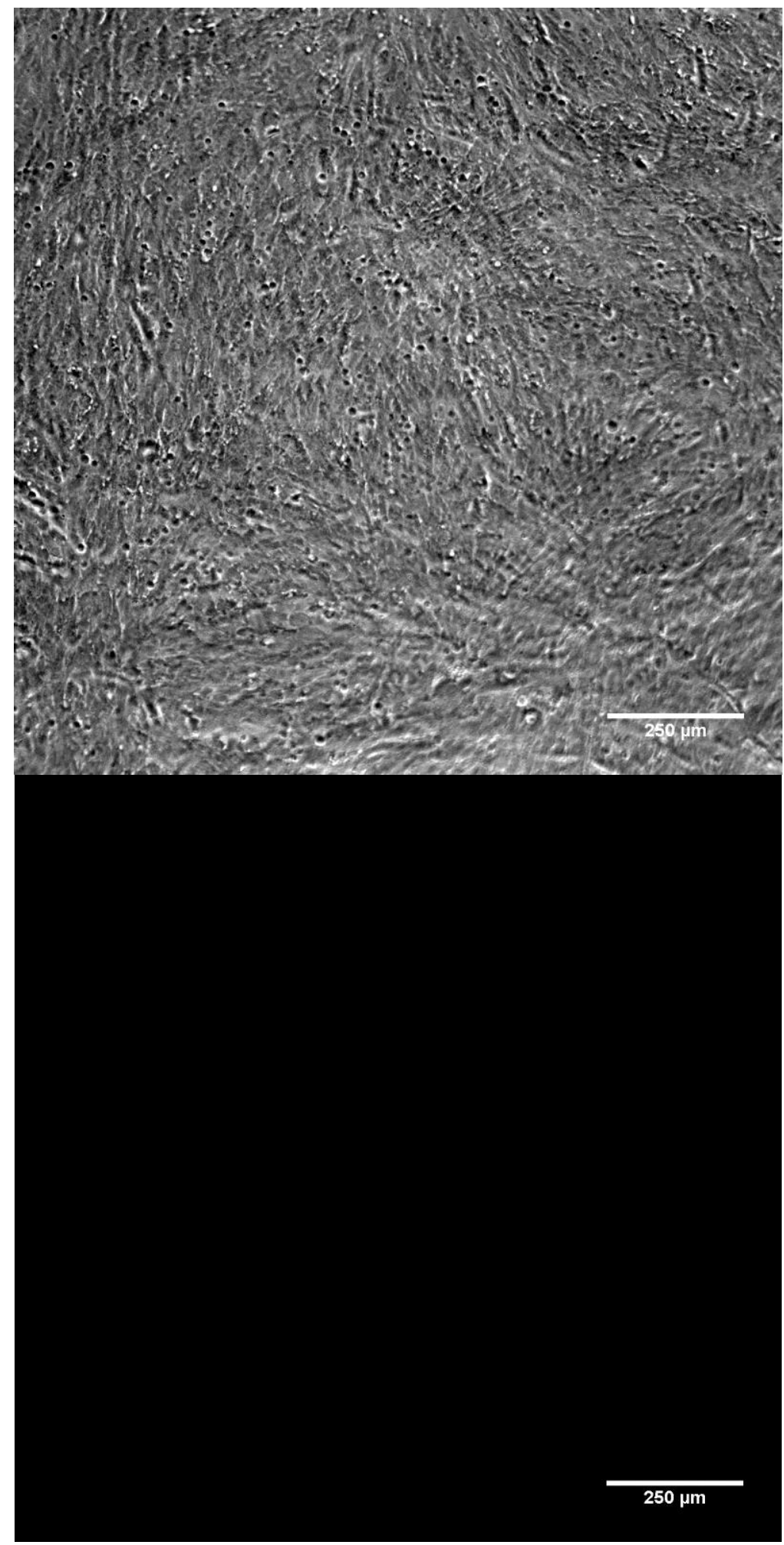

FIGURE A2.44- ARPE FN-3-2 Confluency 


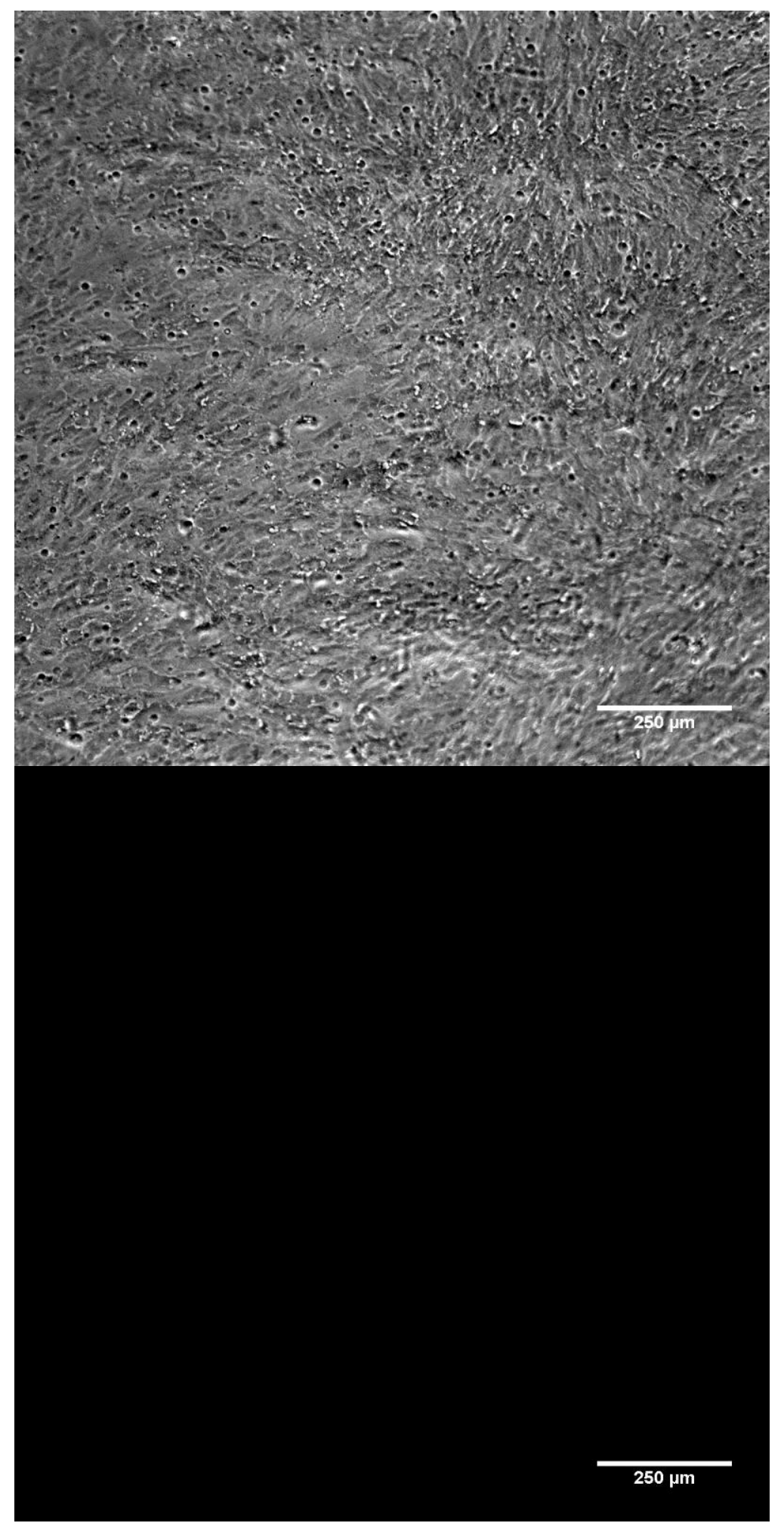

FIGURE A2.45- ARPE FN-3-3 Confluency 


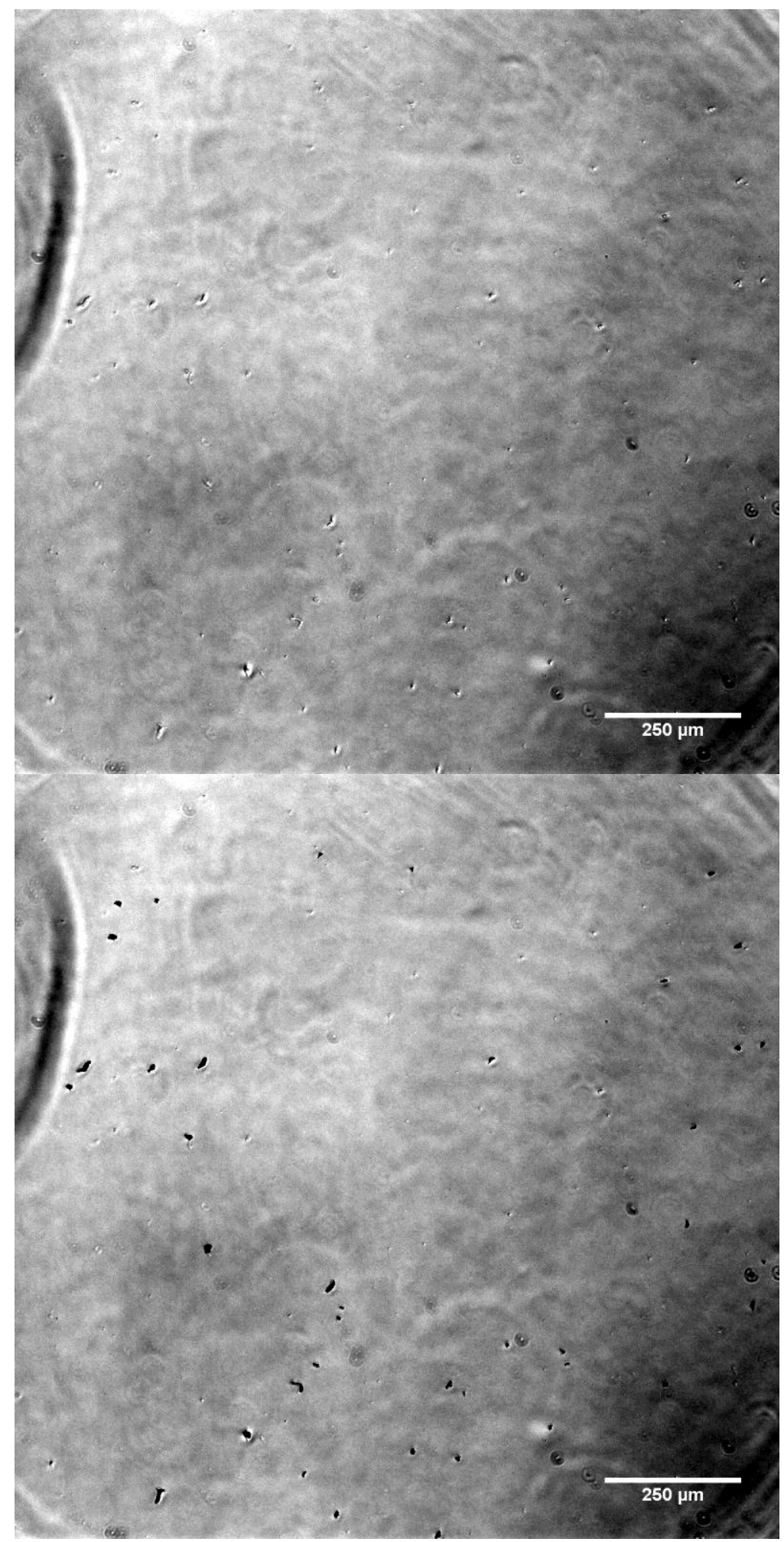

FIGURE A2.46- PRPE 0-1-1 Confluency 


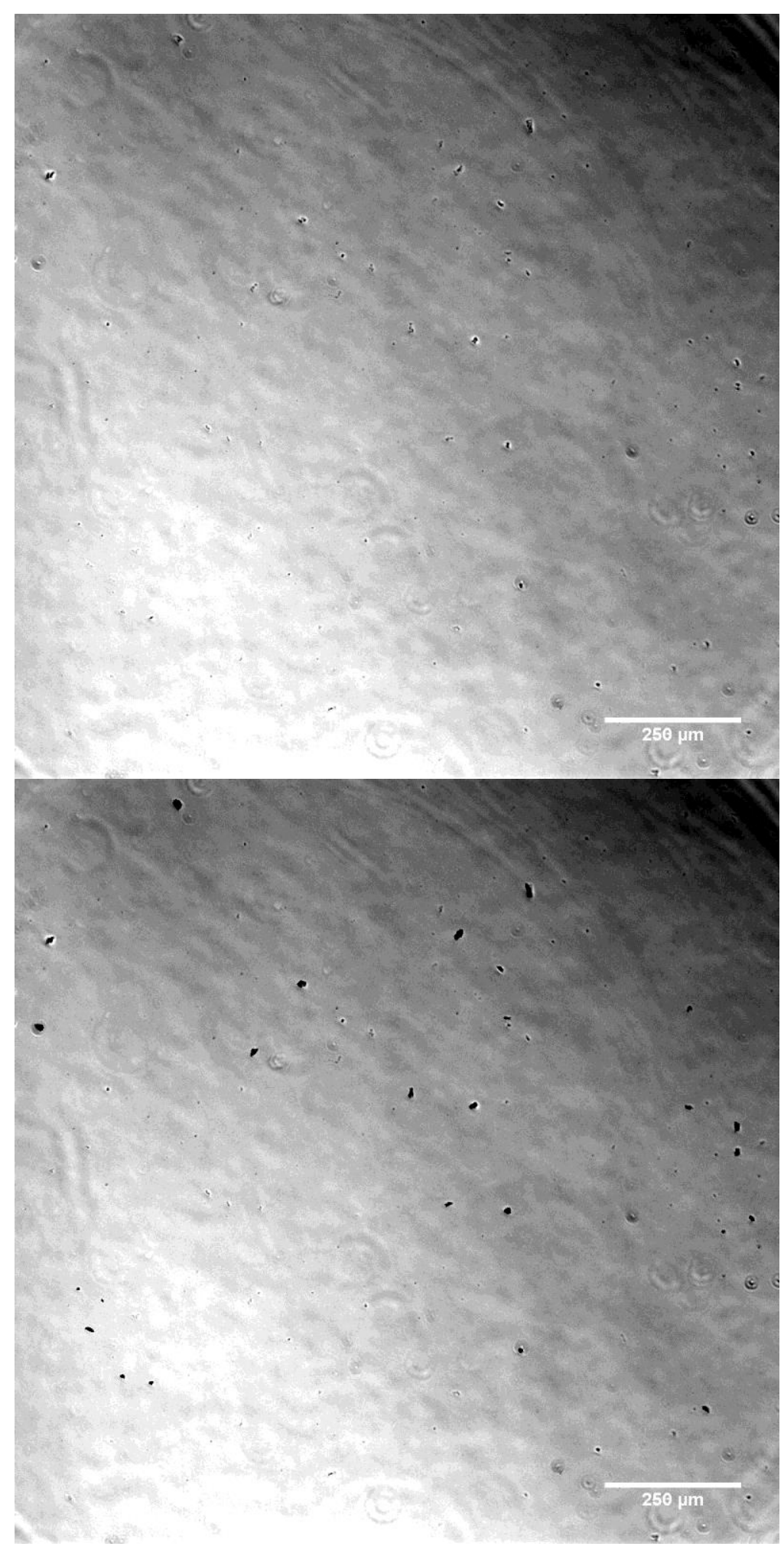

FIGURE A2.47- PRPE 0-1-2 Confluency 


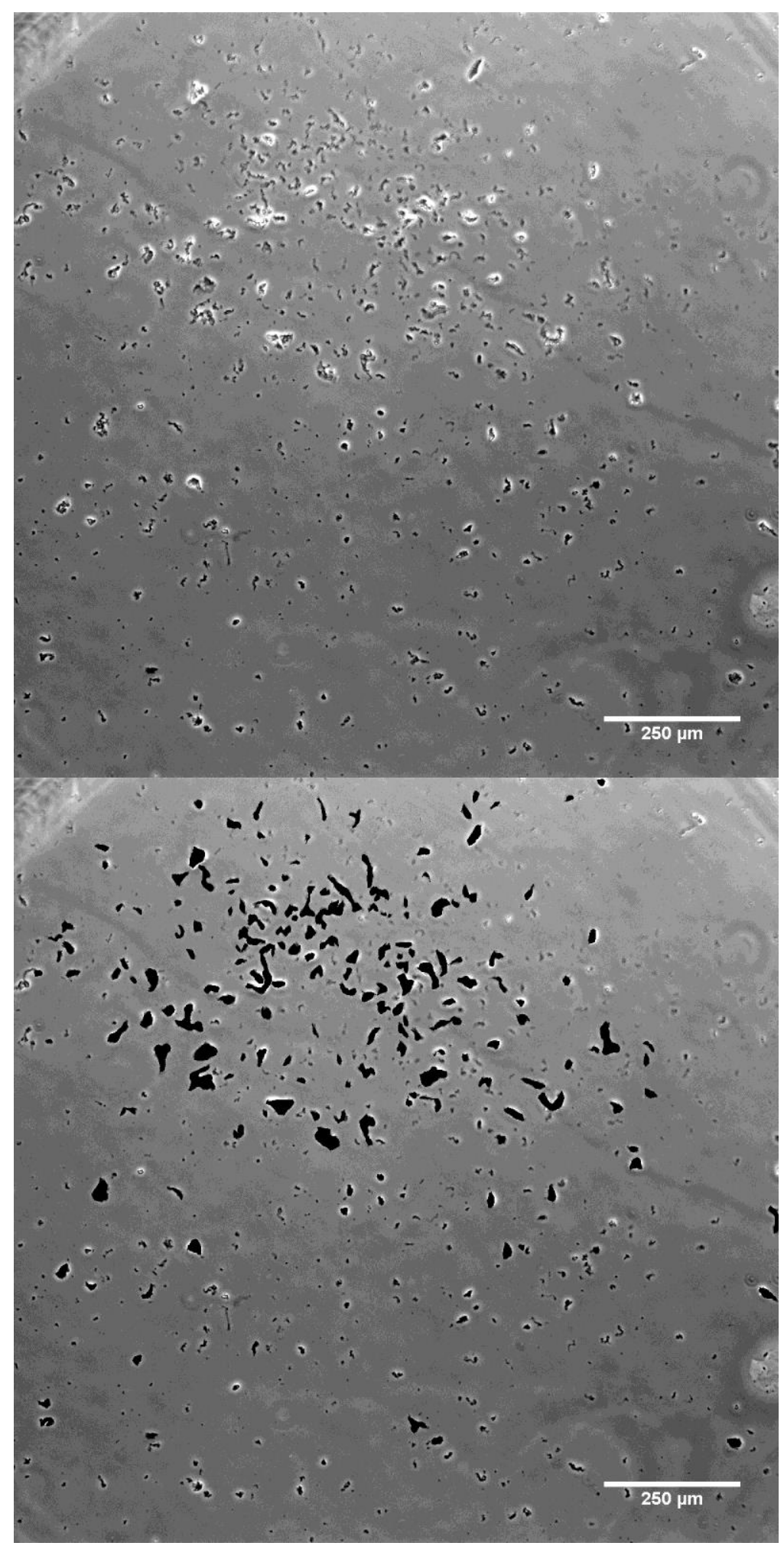

FIGURE A2.48- PRPE 0-1-3 Confluency 


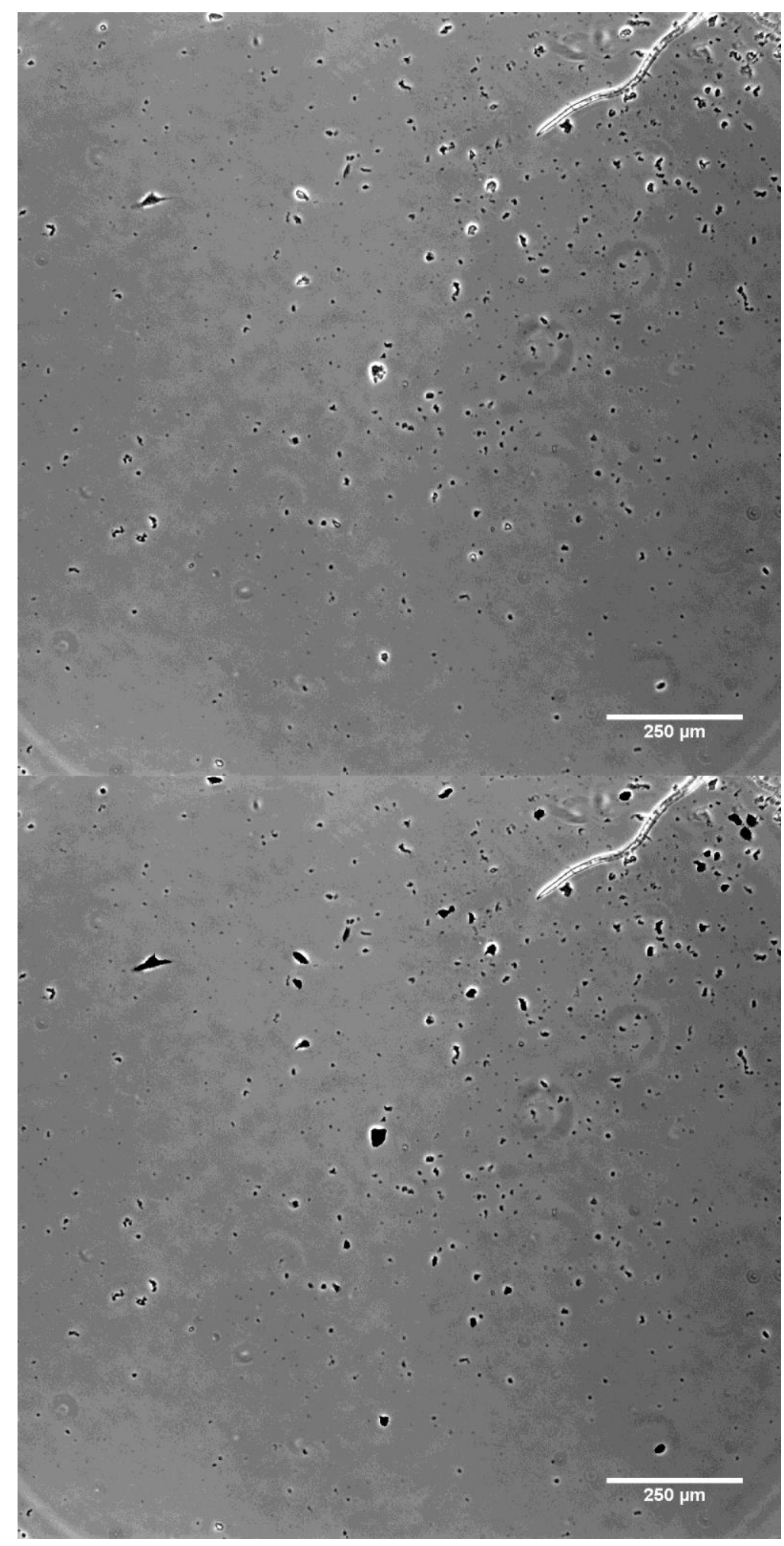

FIGURE A2.49- PRPE 0-2-1 Confluency 


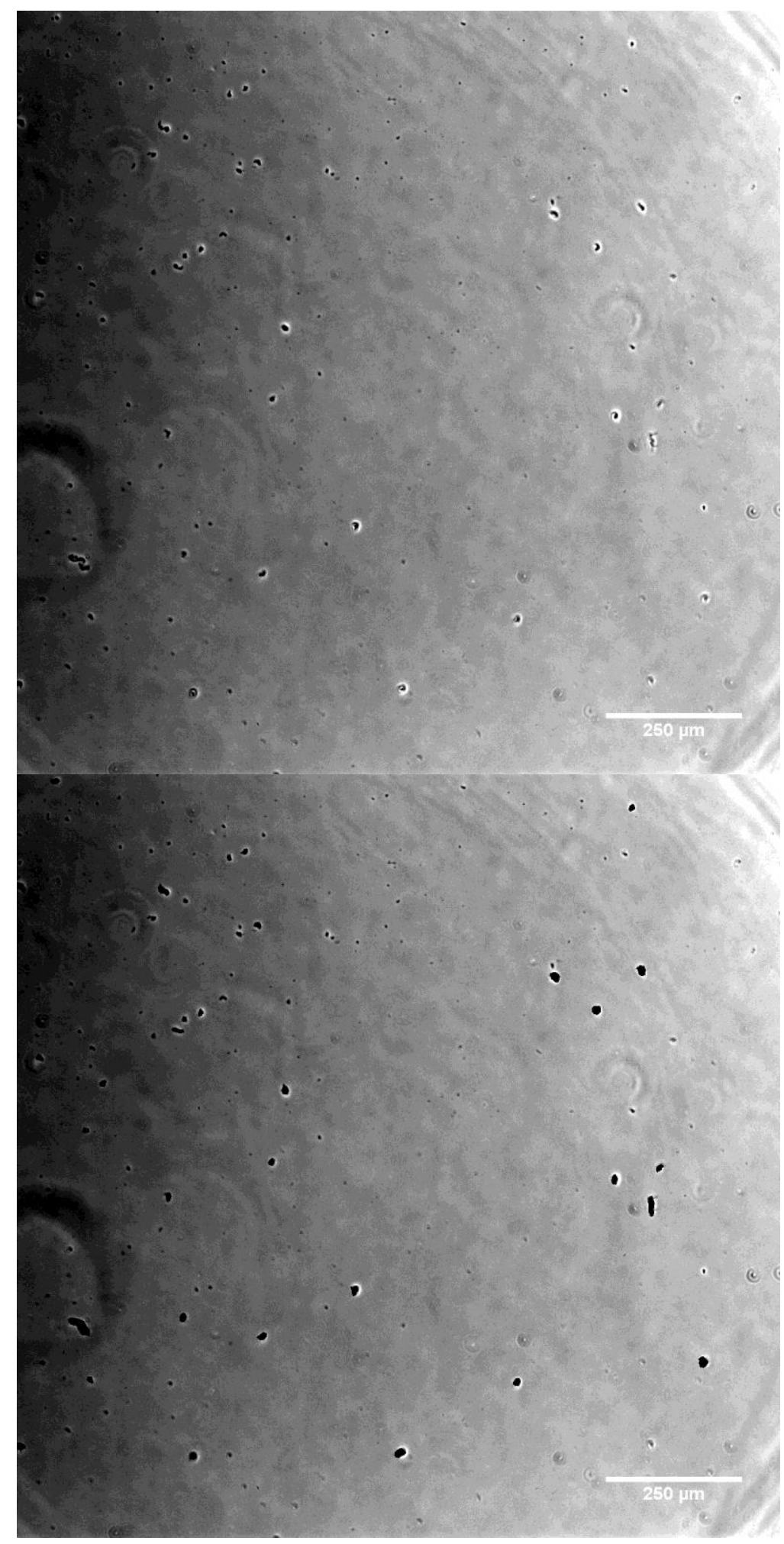

FIGURE A2.50- PRPE 0-2-2 Confluency 


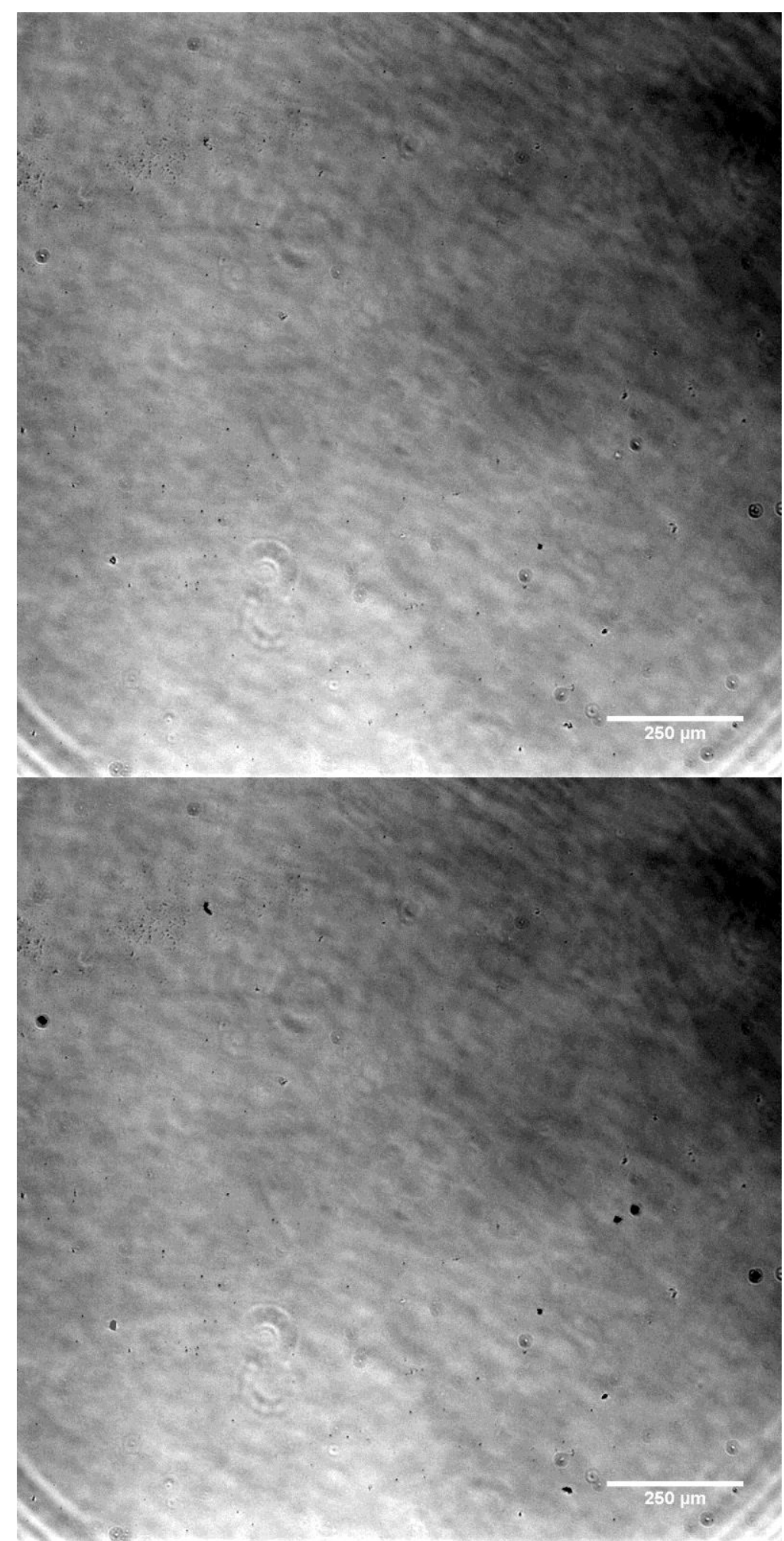

FIGURE A2.51- PRPE 0-2-3 Confluency 


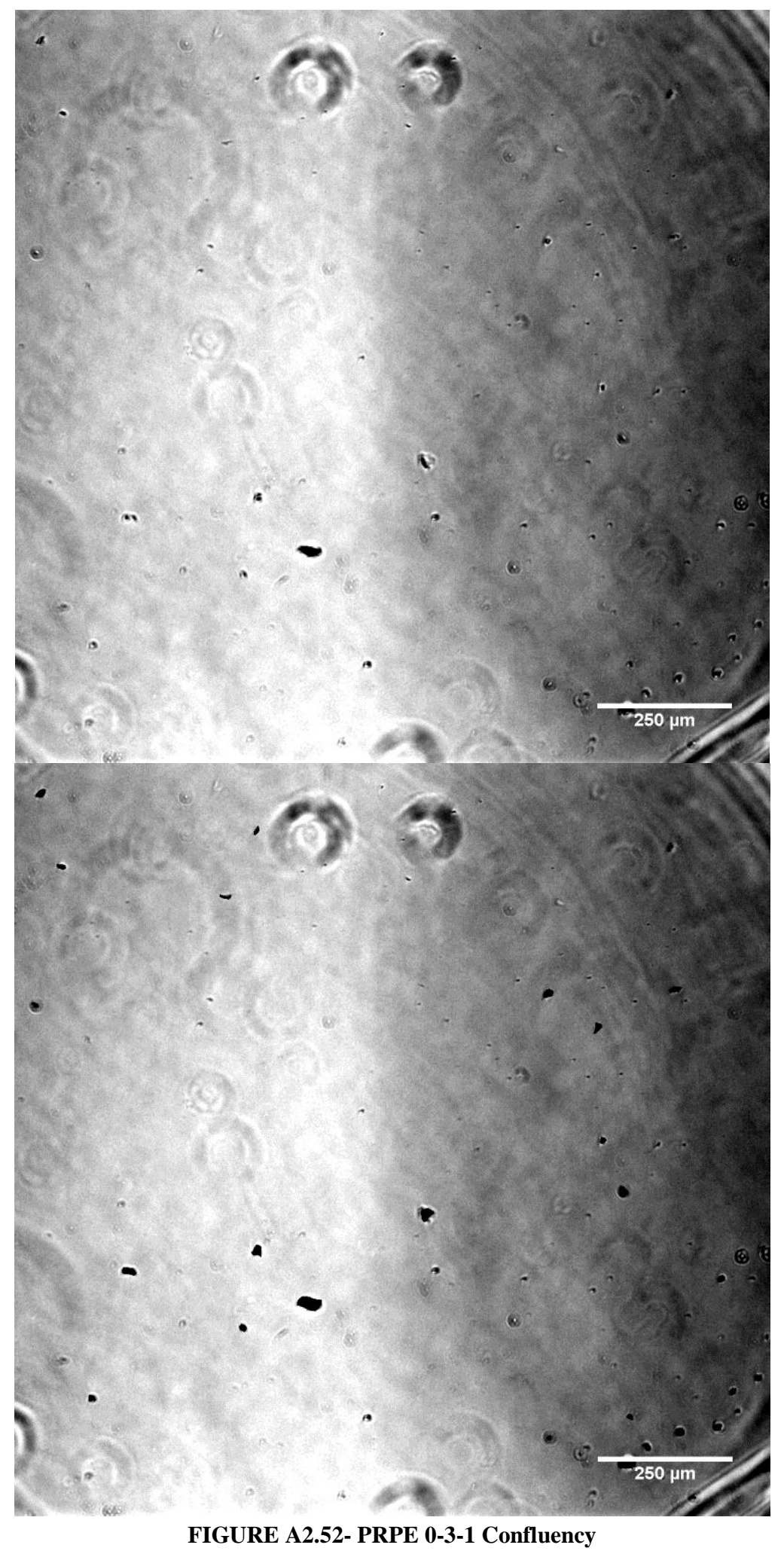




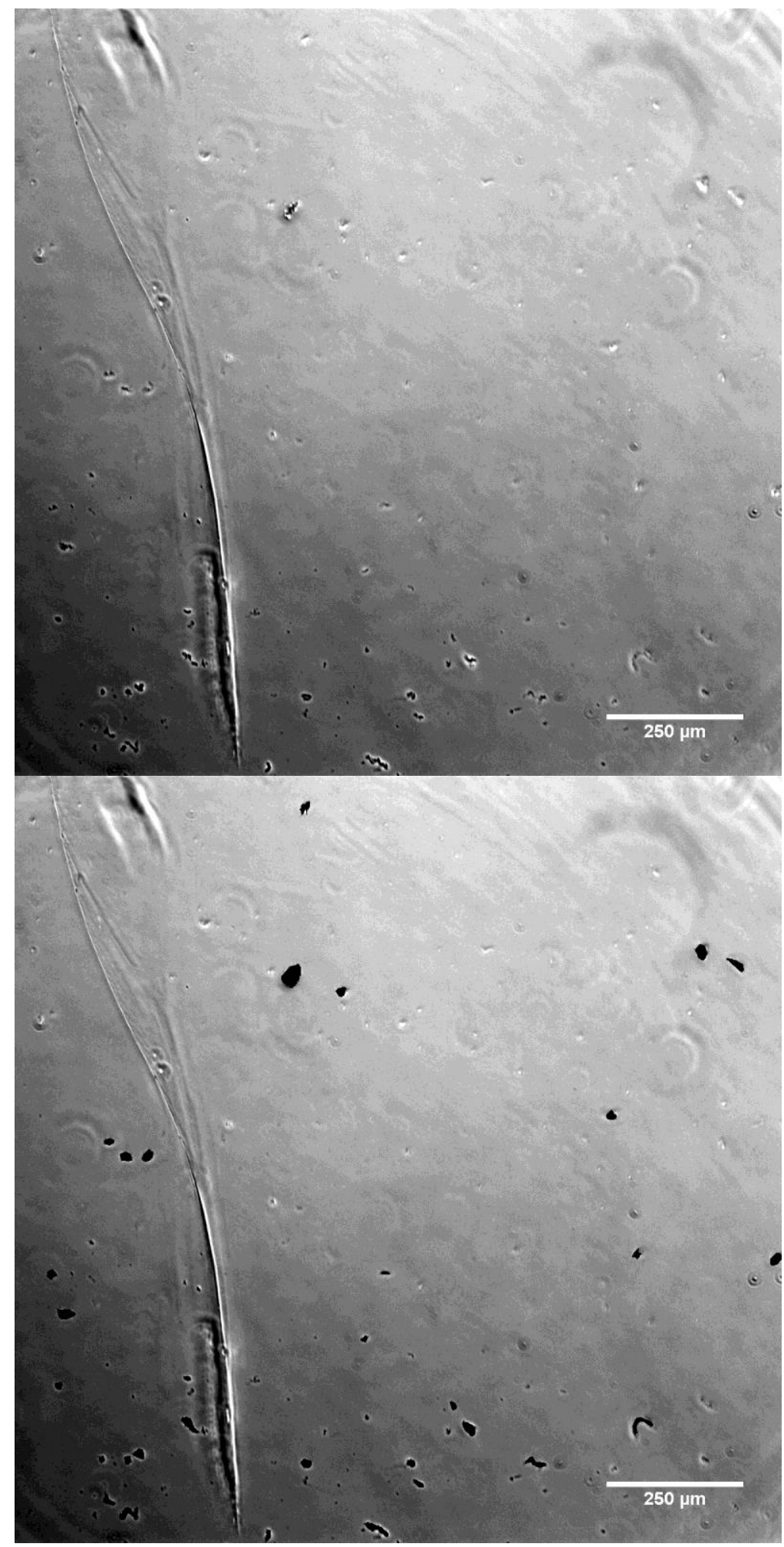

FIGURE A2.53- PRPE 0-3-2 Confluency 


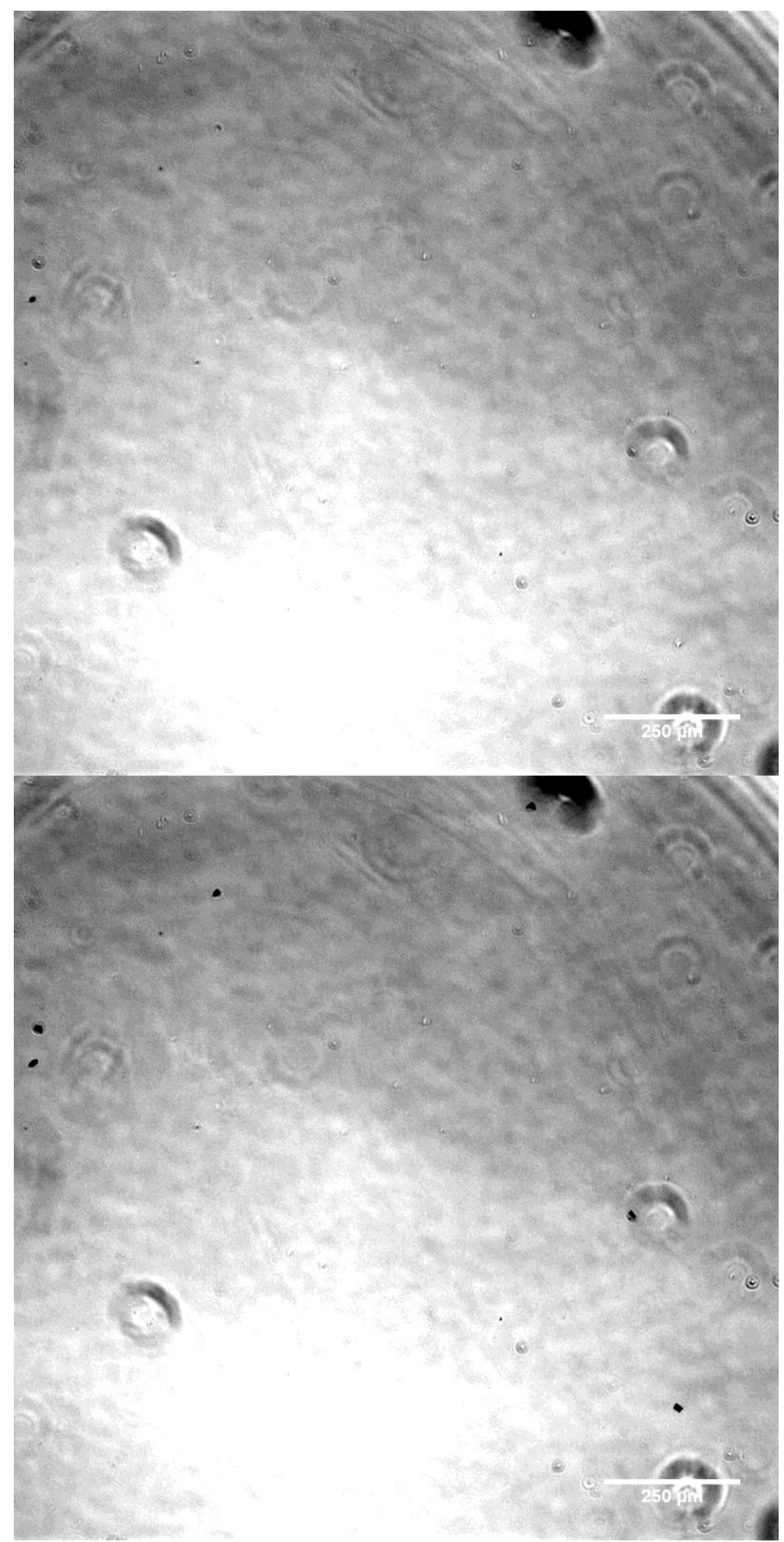

FIGURE A2.54- PRPE 0-3-3 Confluency 


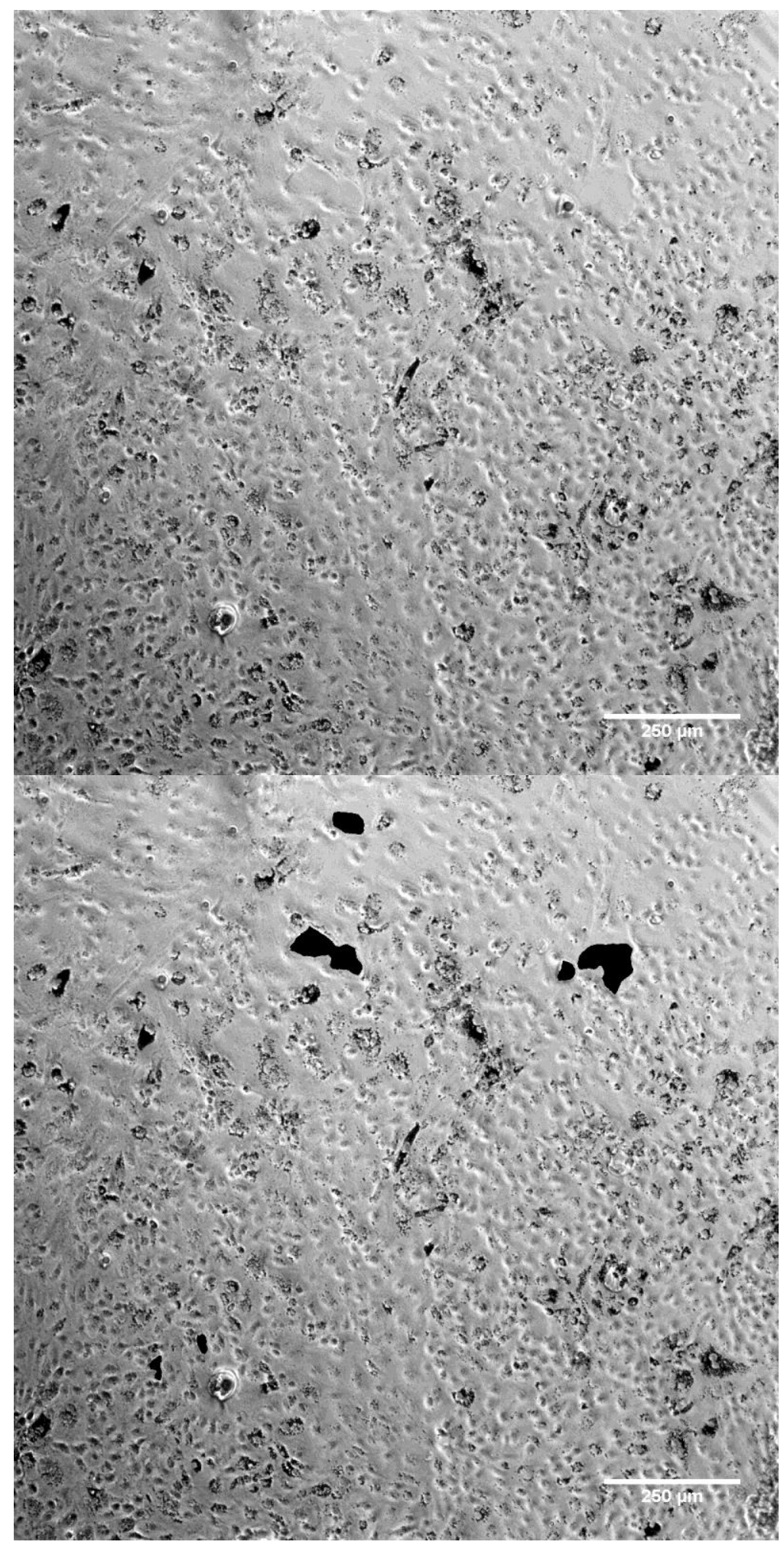

FIGURE A2.55- PRPE 5-1-1 Confluency 


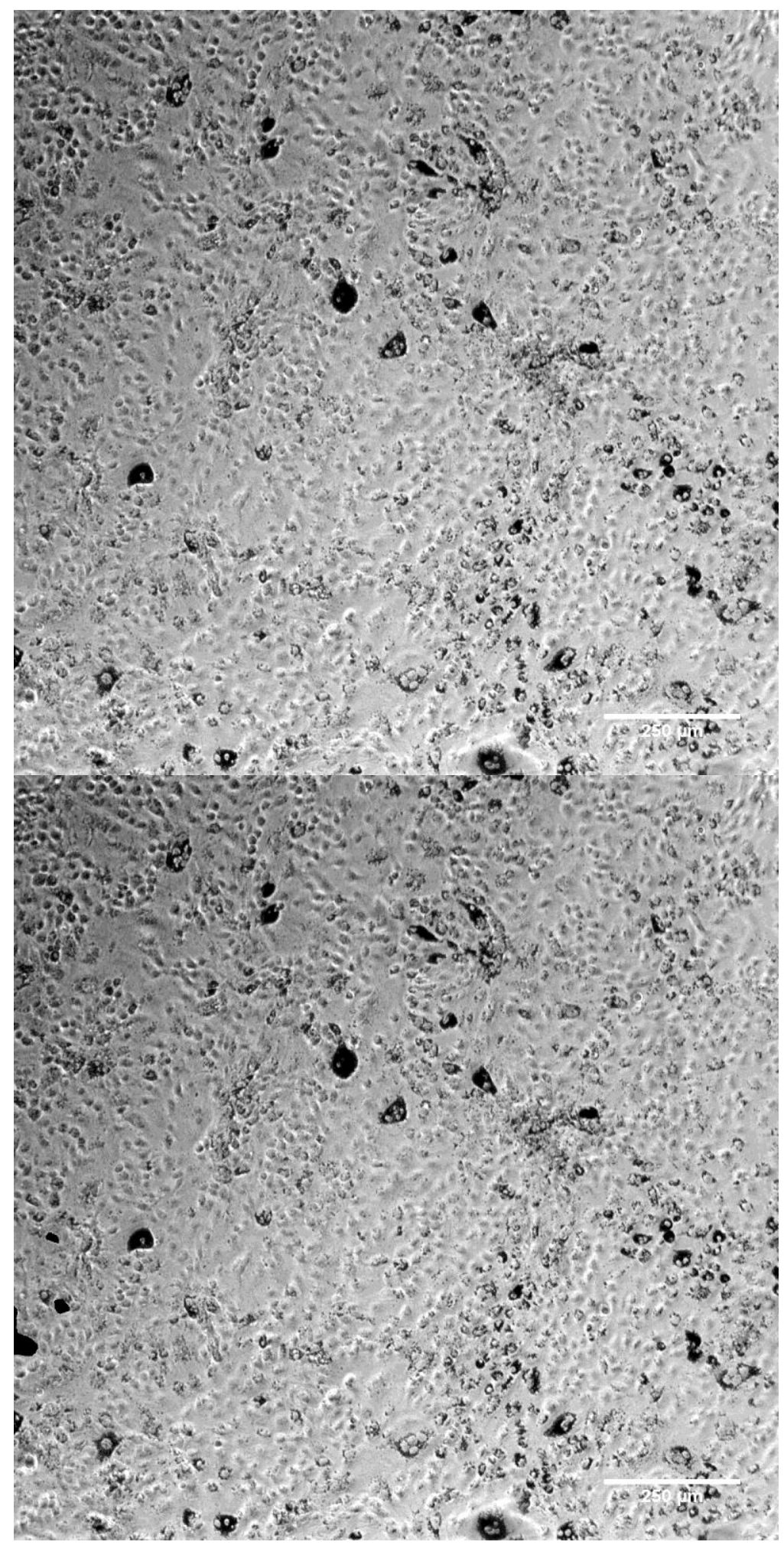

FIGURE A2.56- PRPE 5-1-2 Confluency 


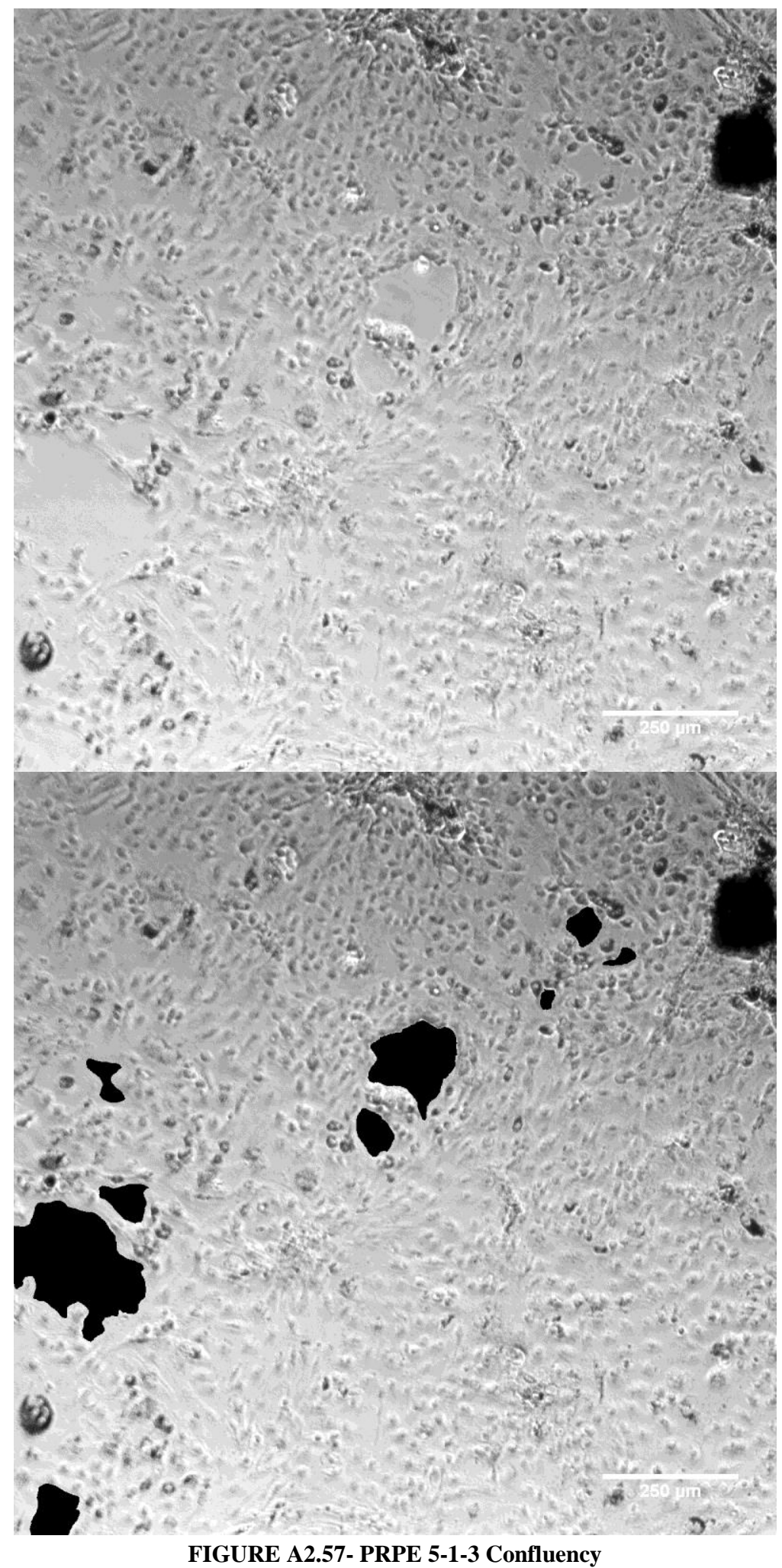




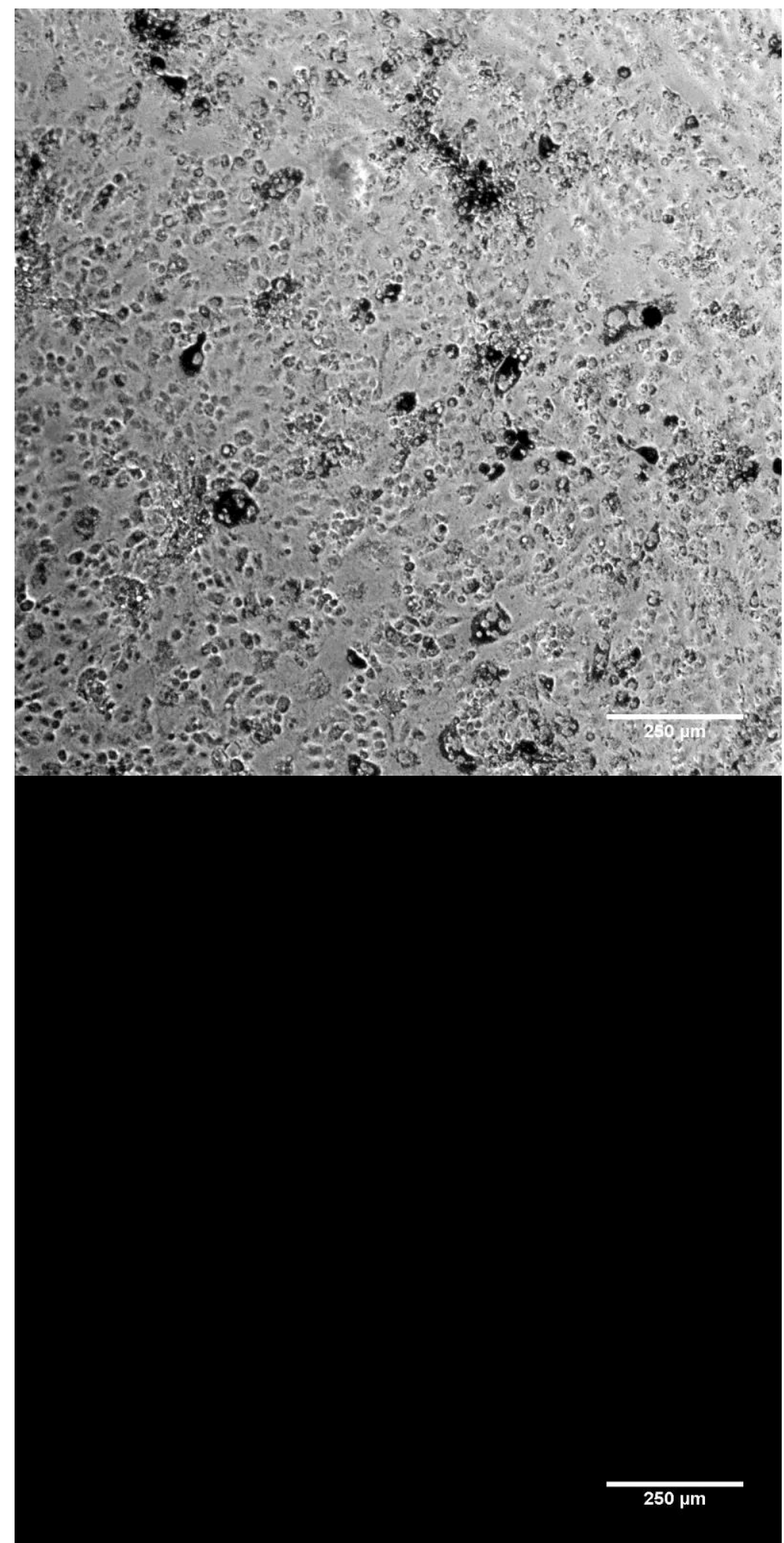

FIGURE A2.58- PRPE 5-2-1 Confluency 


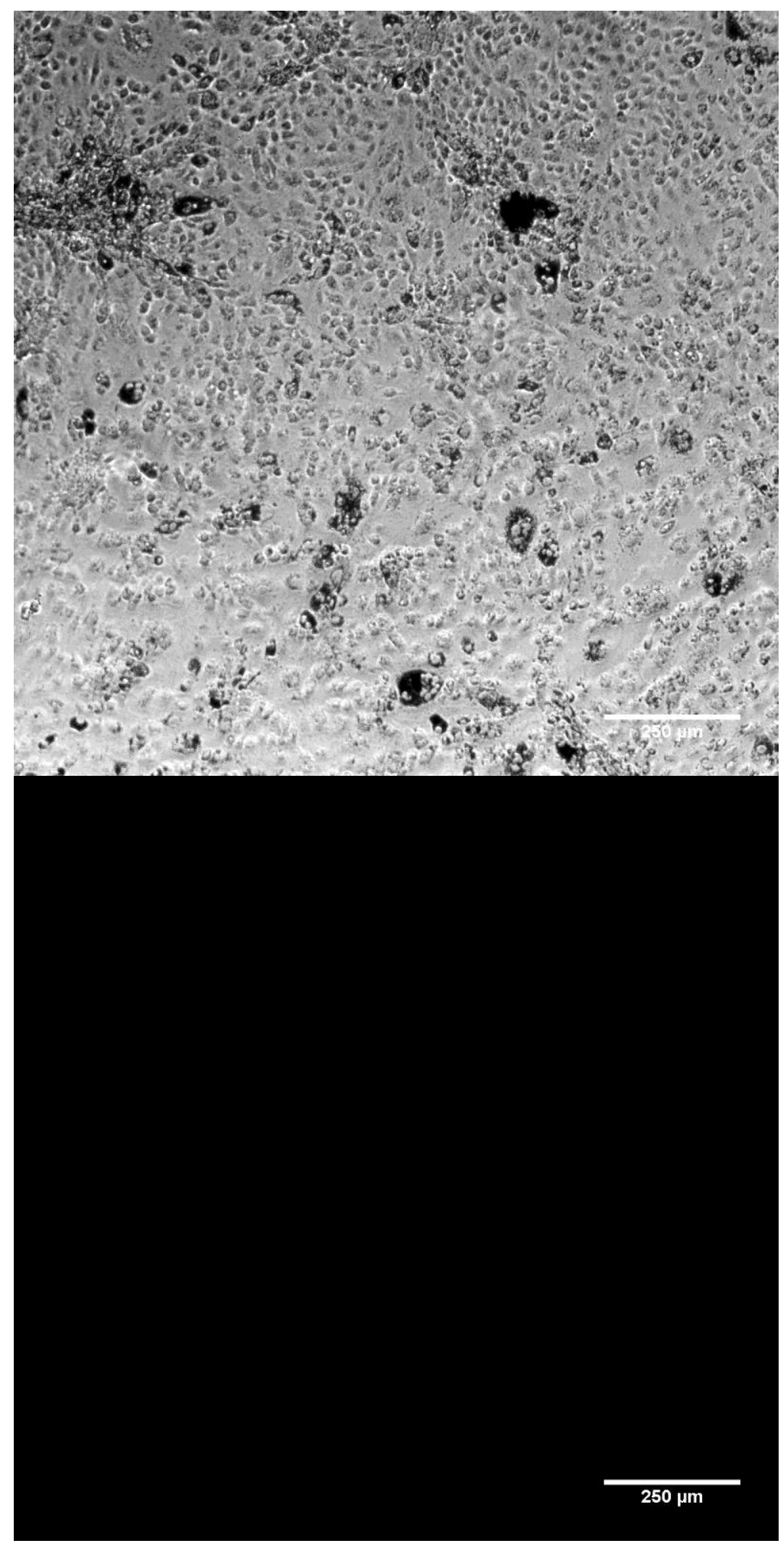

FIGURE A2.59- PRPE 5-2-2 Confluency 


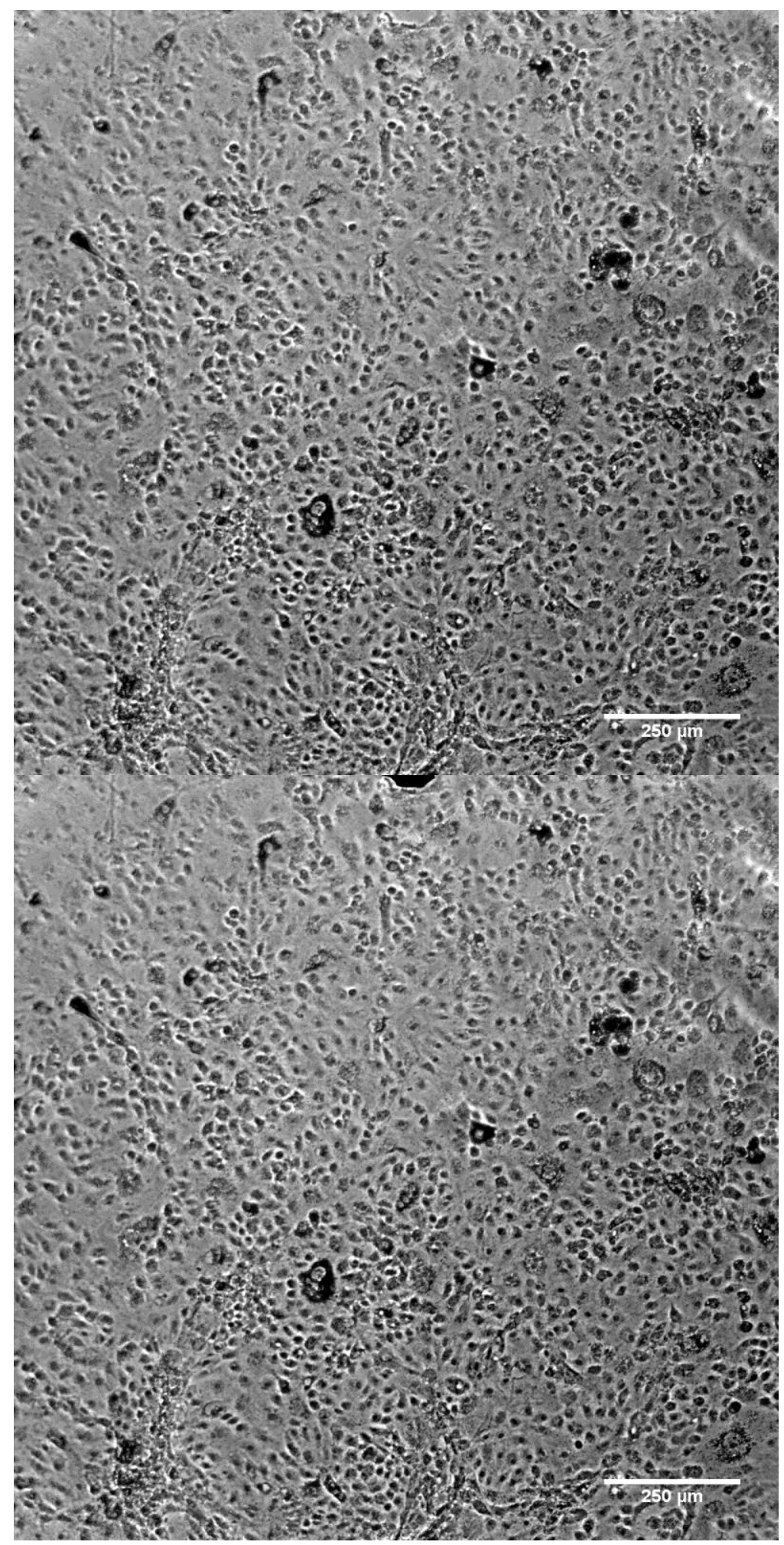

FIGURE A2.60- PRPE 5-2-3 Confluency 


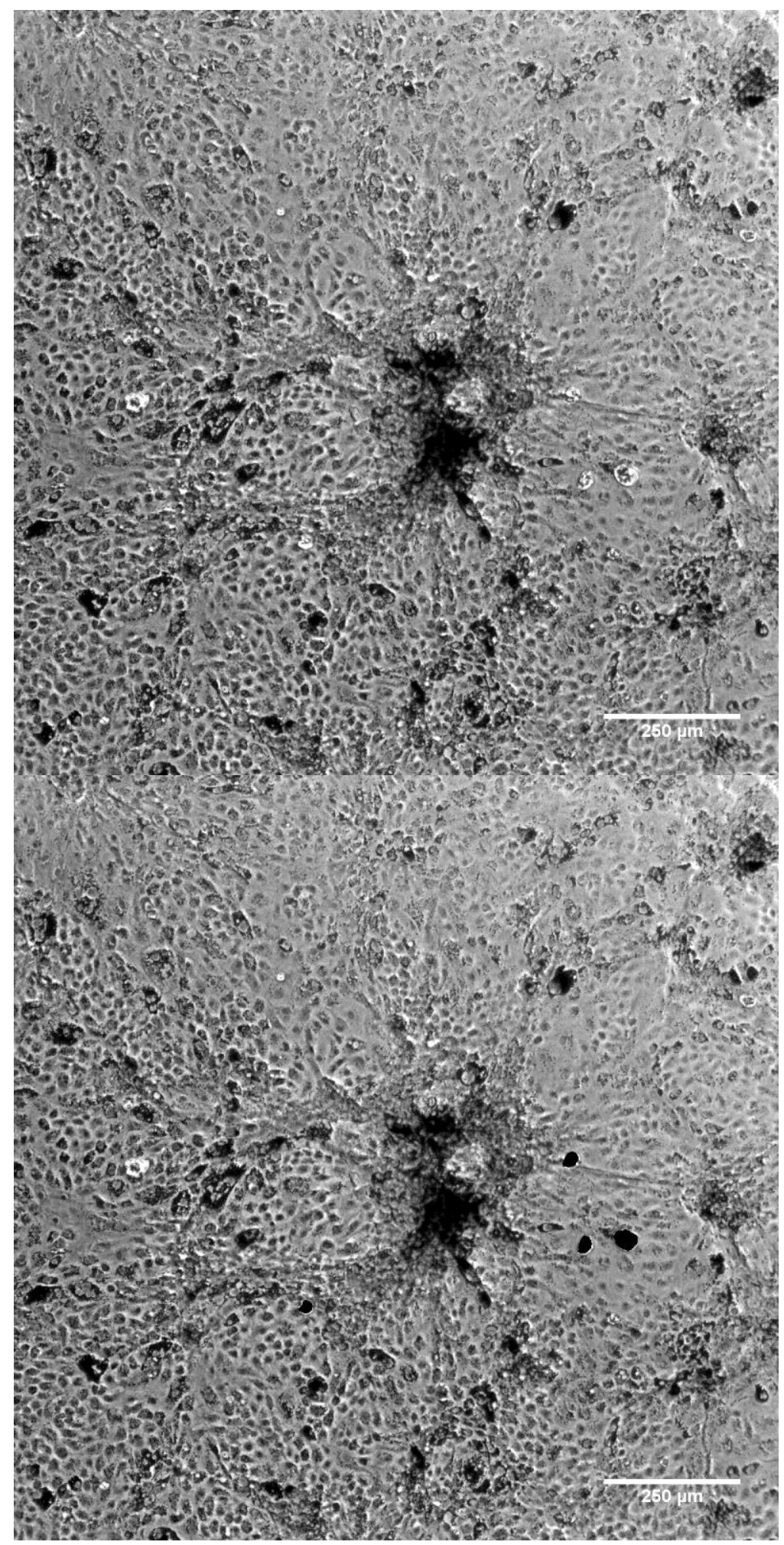

FIGURE A2.61- PRPE 5-3-1 Confluency 


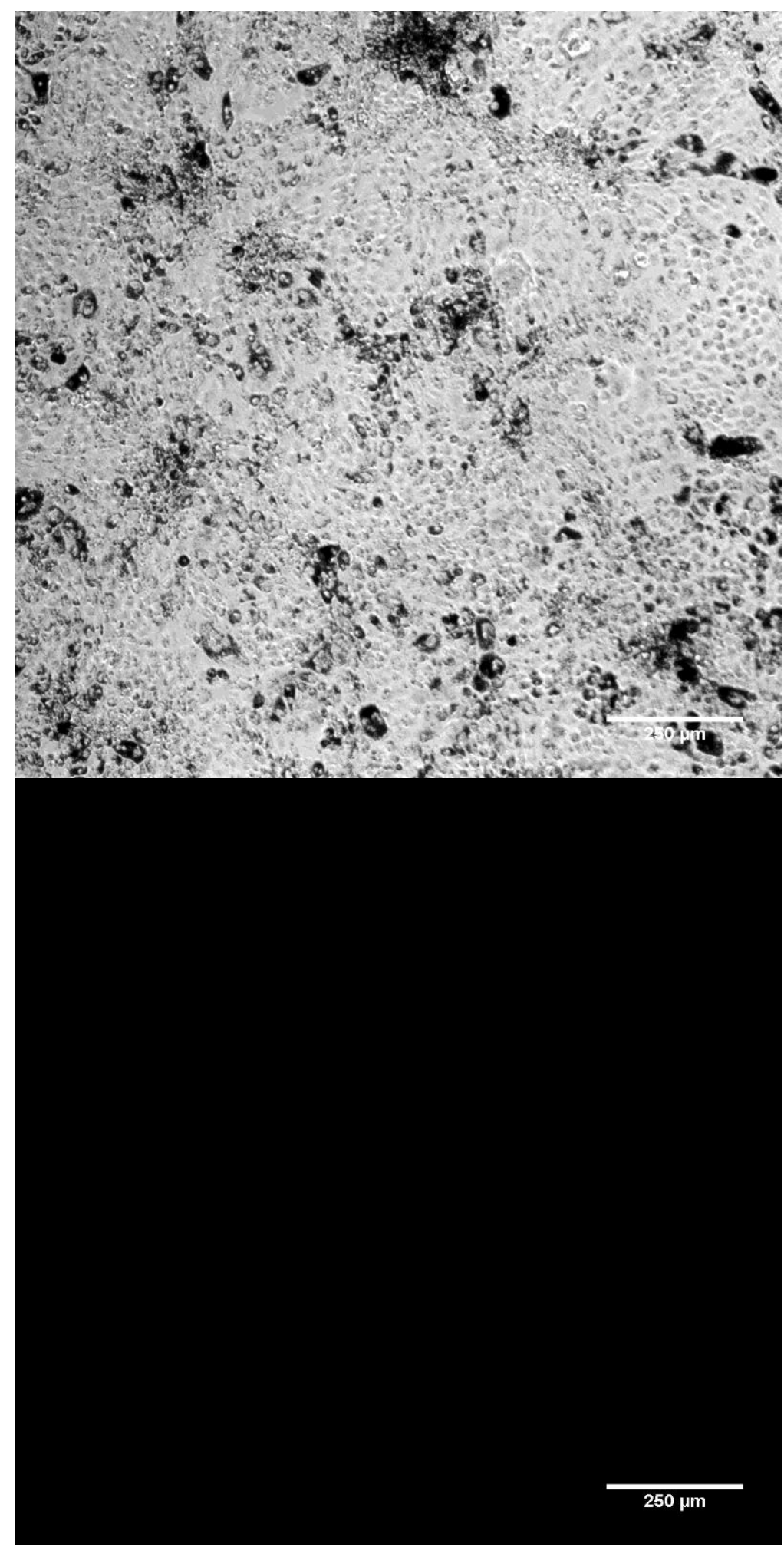

FIGURE A2.62- PRPE 5-3-2 Confluency 


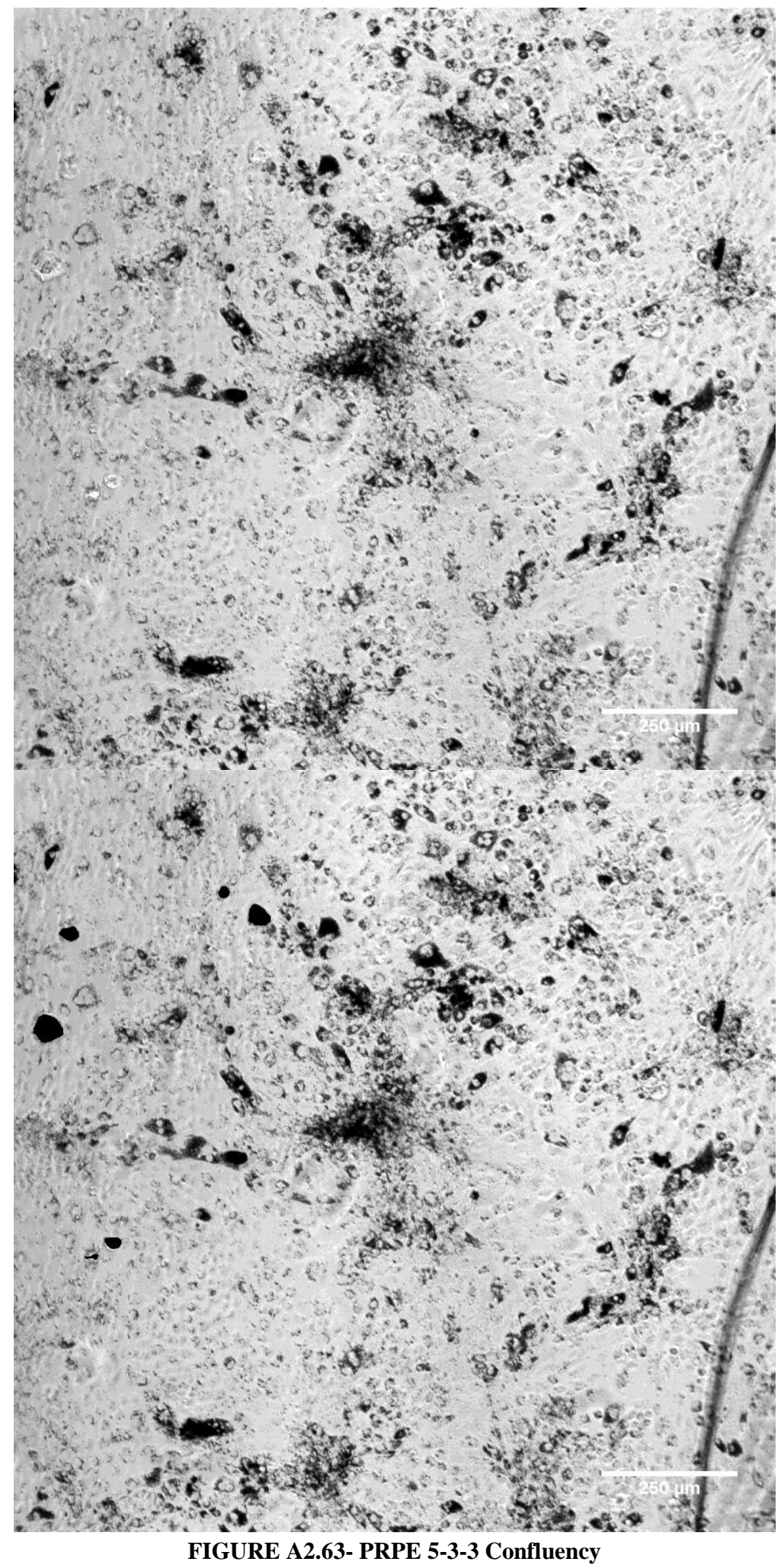




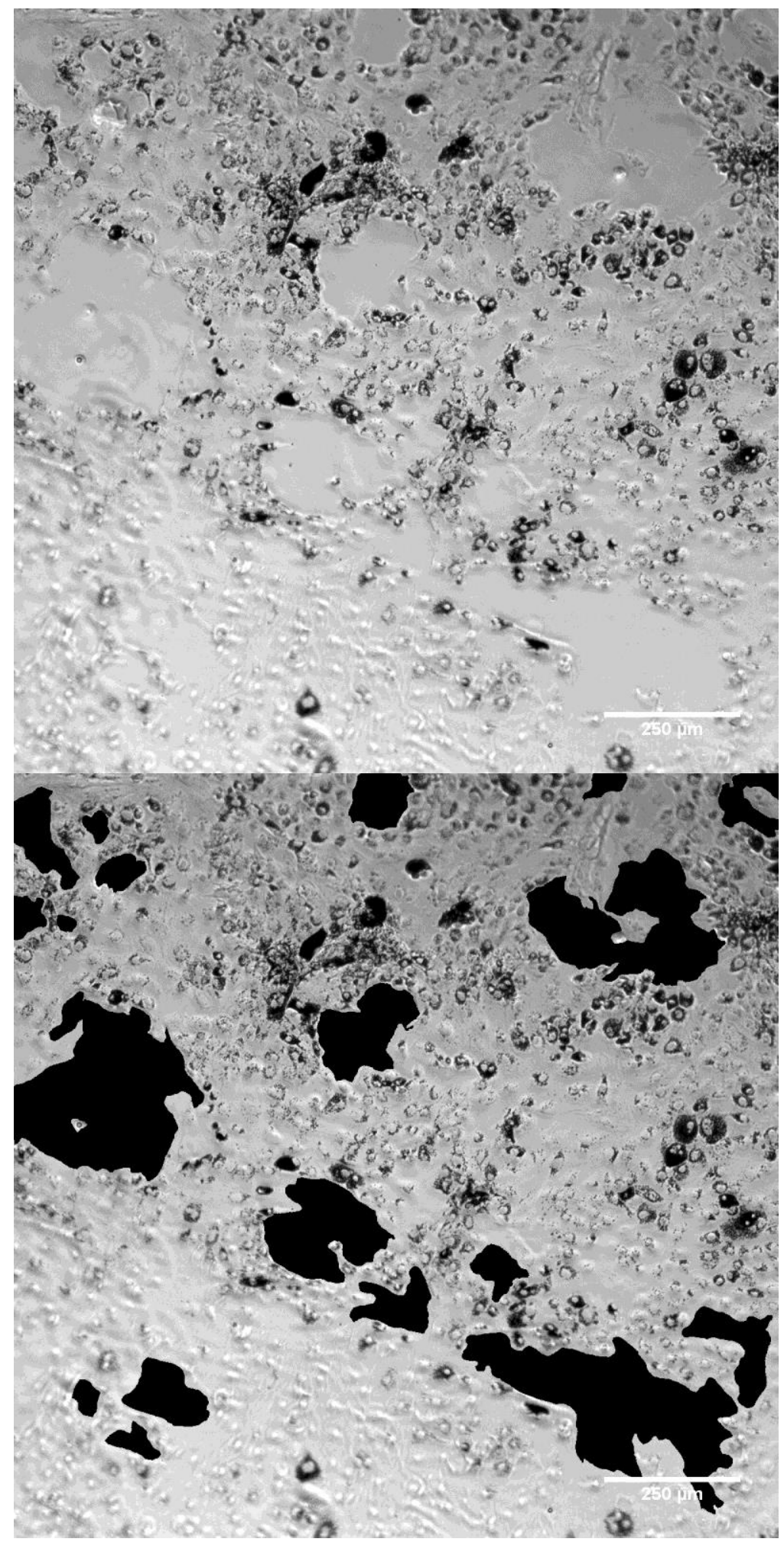

FIGURE A2.64- PRPE 10-1-1 Confluency 


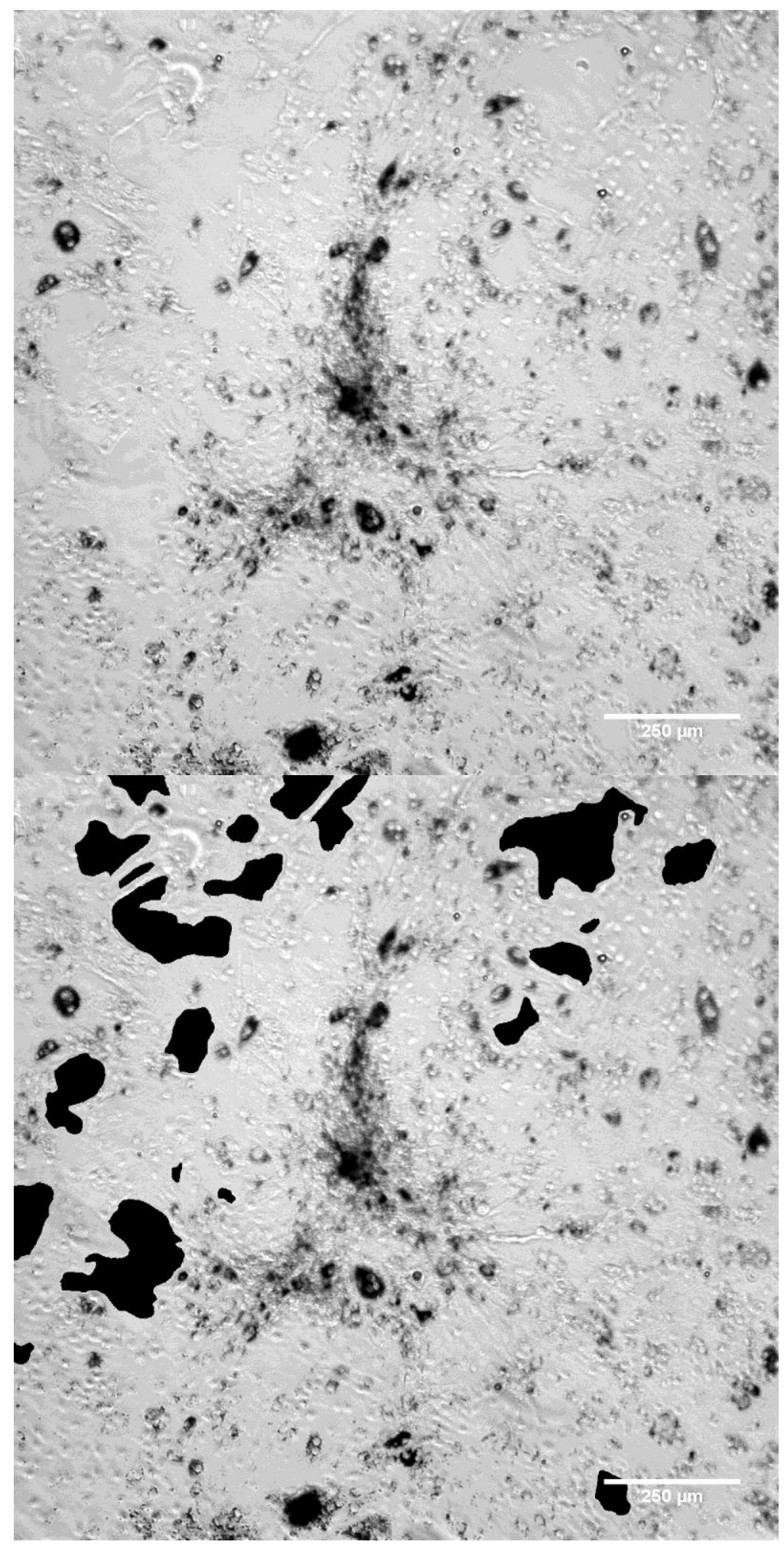

FIGURE A2.65- PRPE 10-1-2 Confluency 


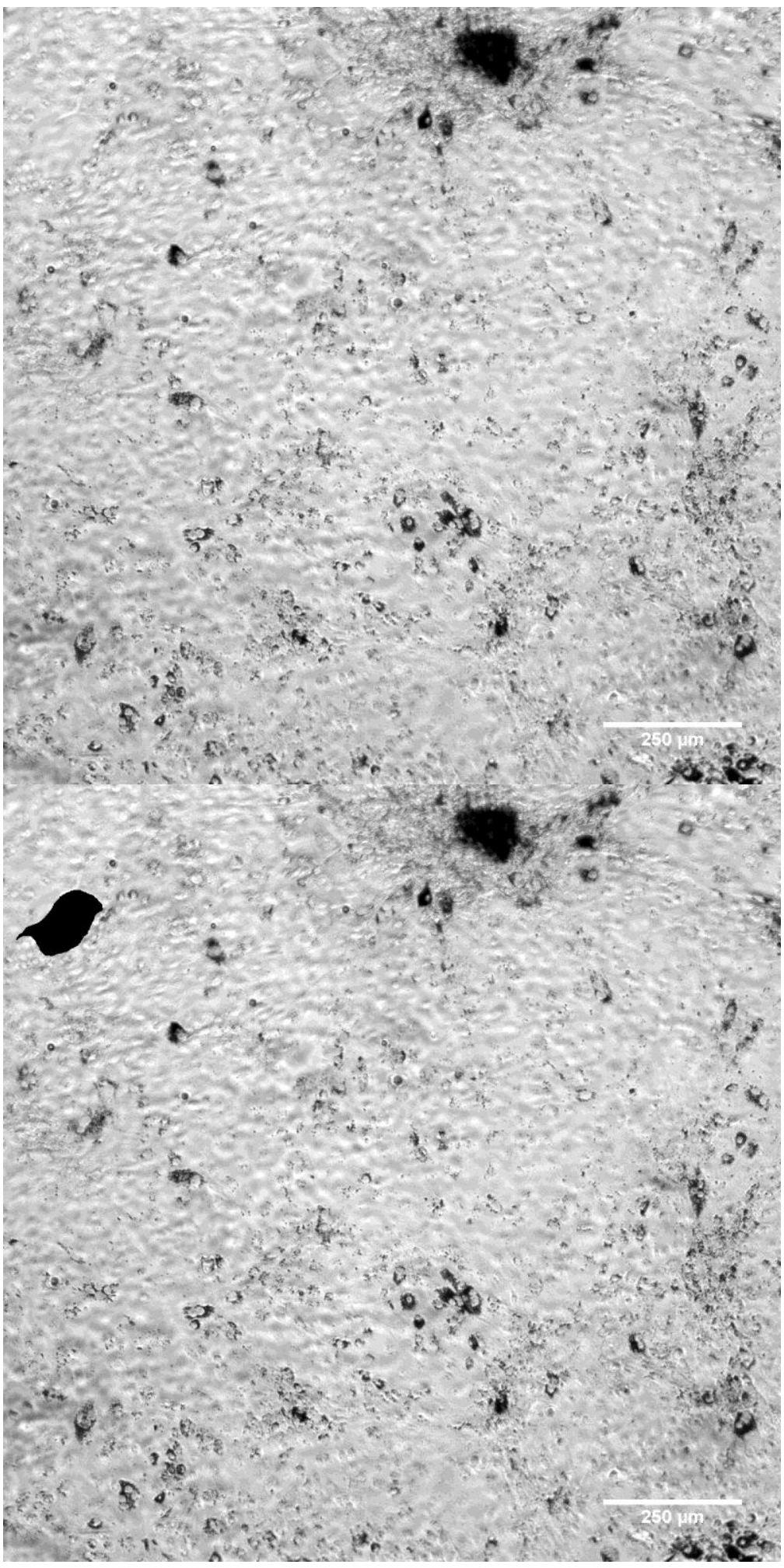

FIGURE A2.66- PRPE 10-1-3 Confluency 


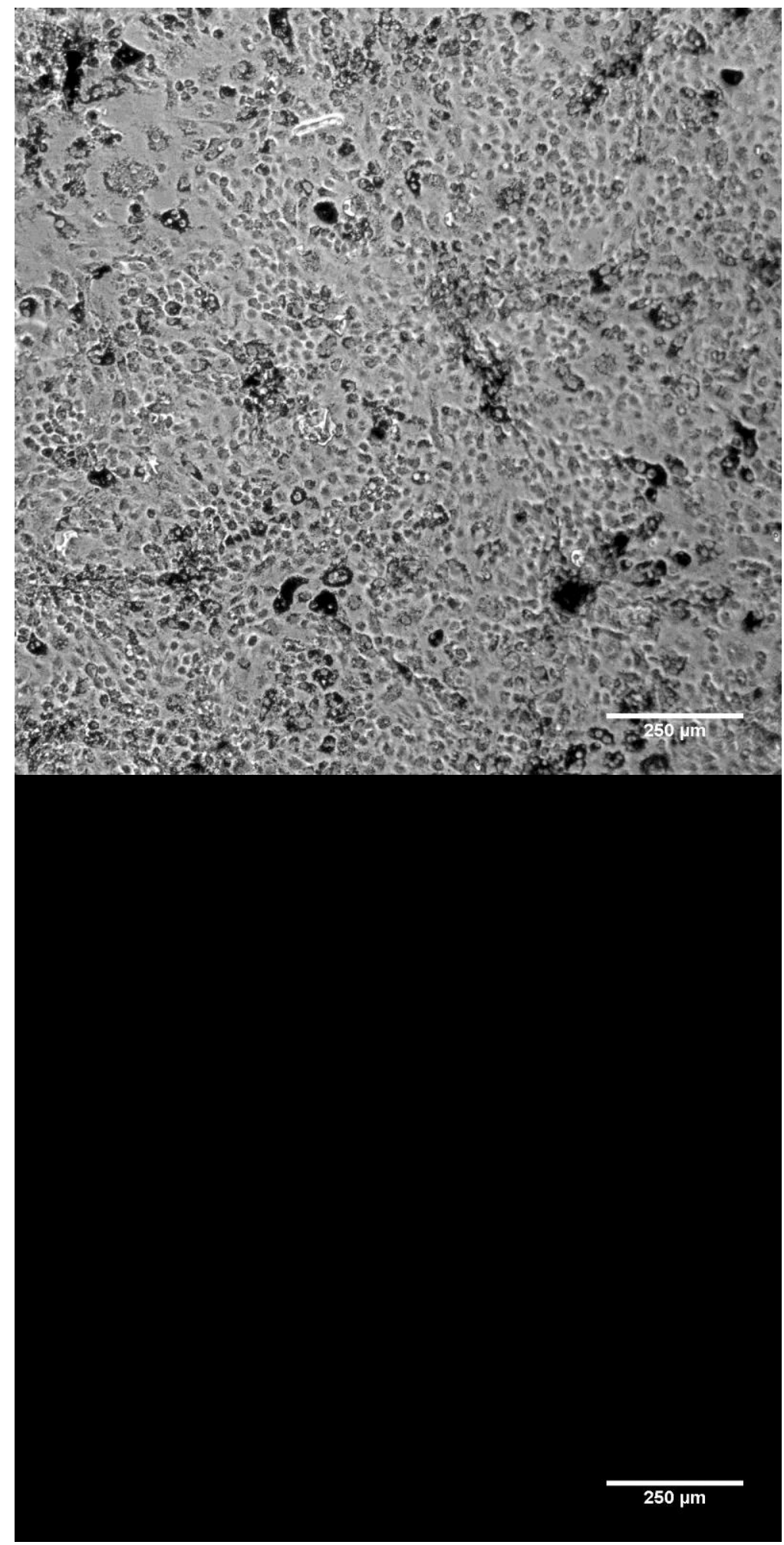

FIGURE A2.67- PRPE 10-2-1 Confluency 


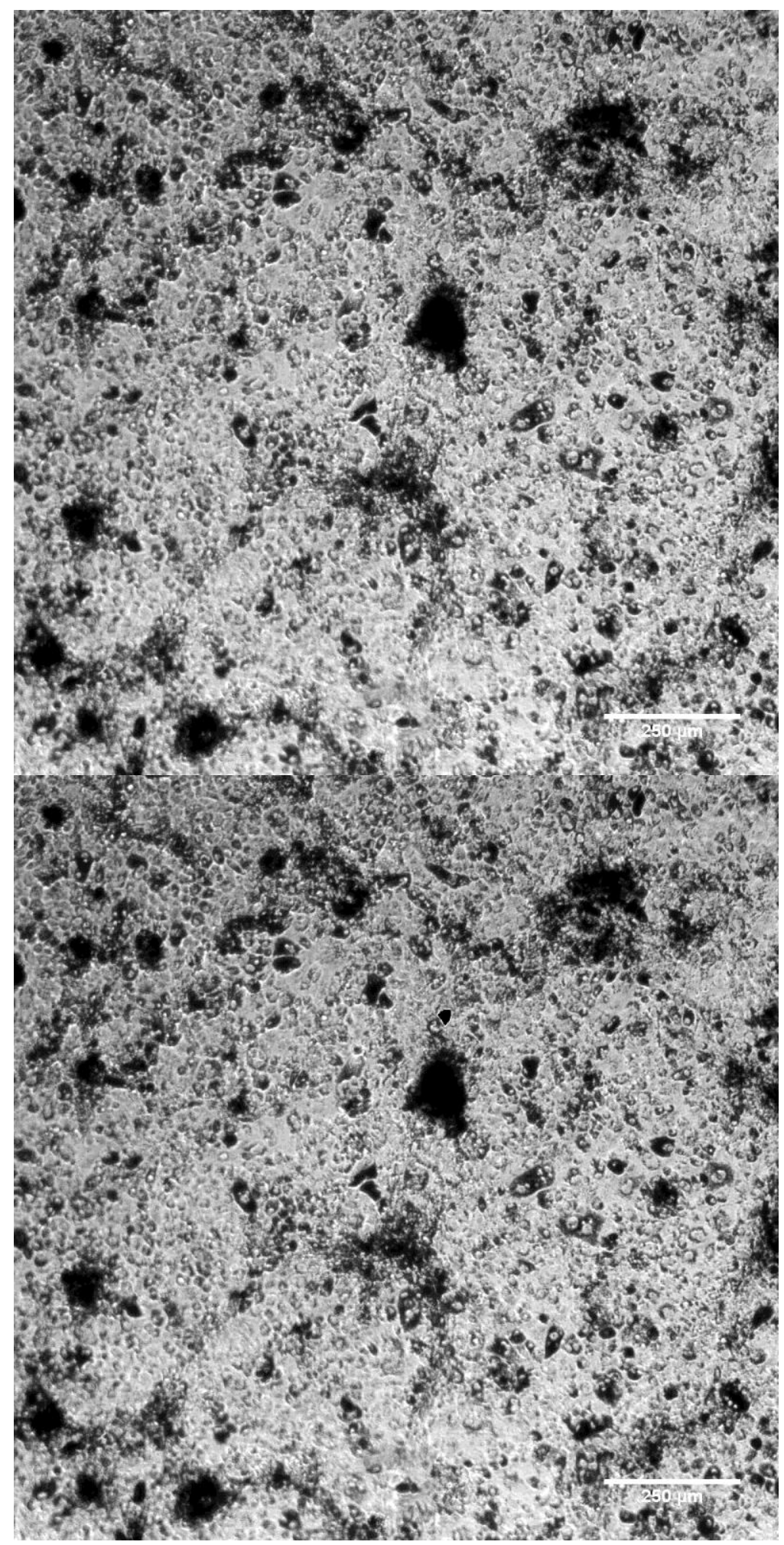

FIGURE A2.68- PRPE 10-2-2 Confluency 


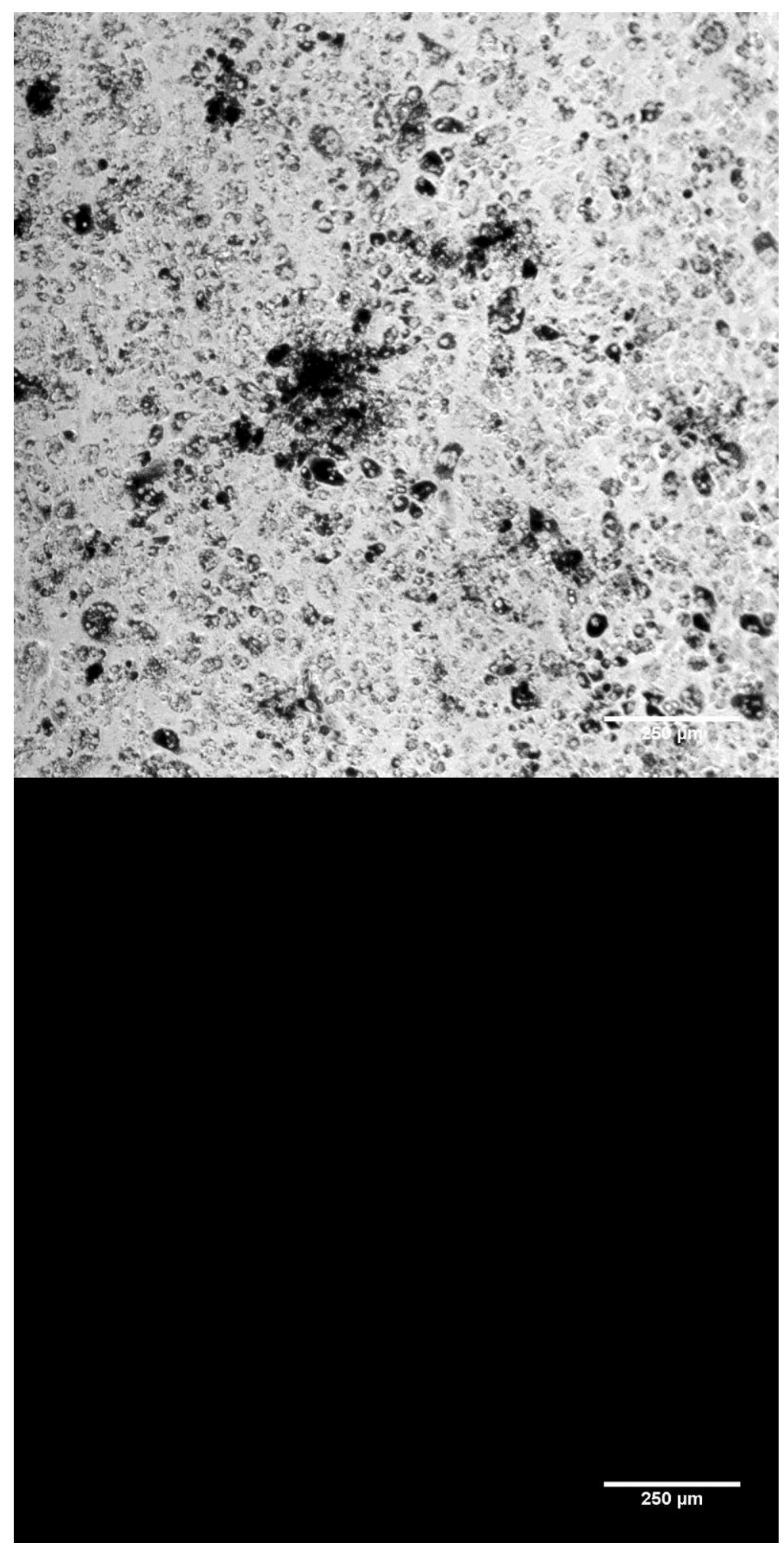

FIGURE A2.69- PRPE 10-2-3 Confluency 


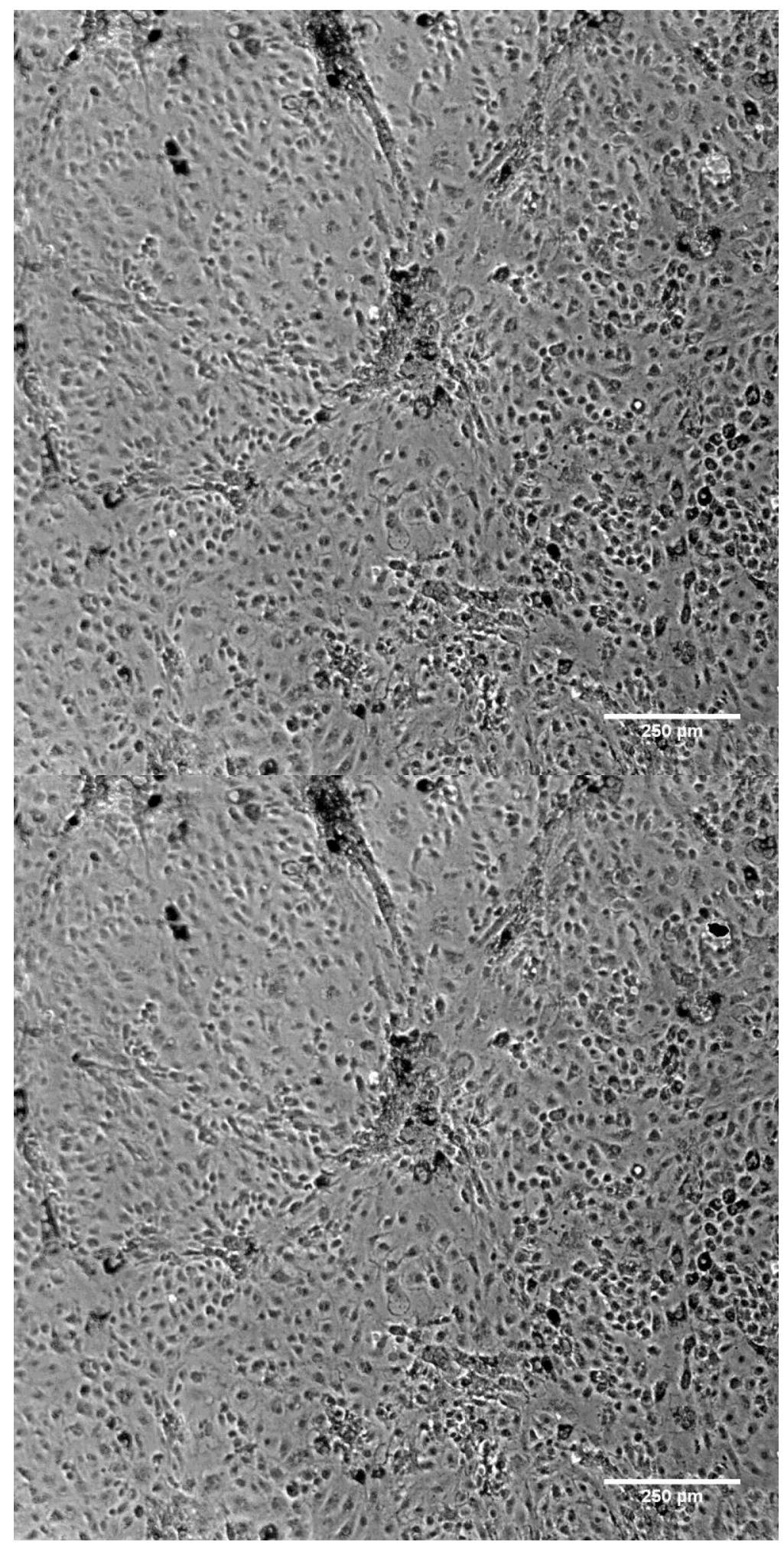

FIGURE A2.70- PRPE 10-3-1 Confluency 


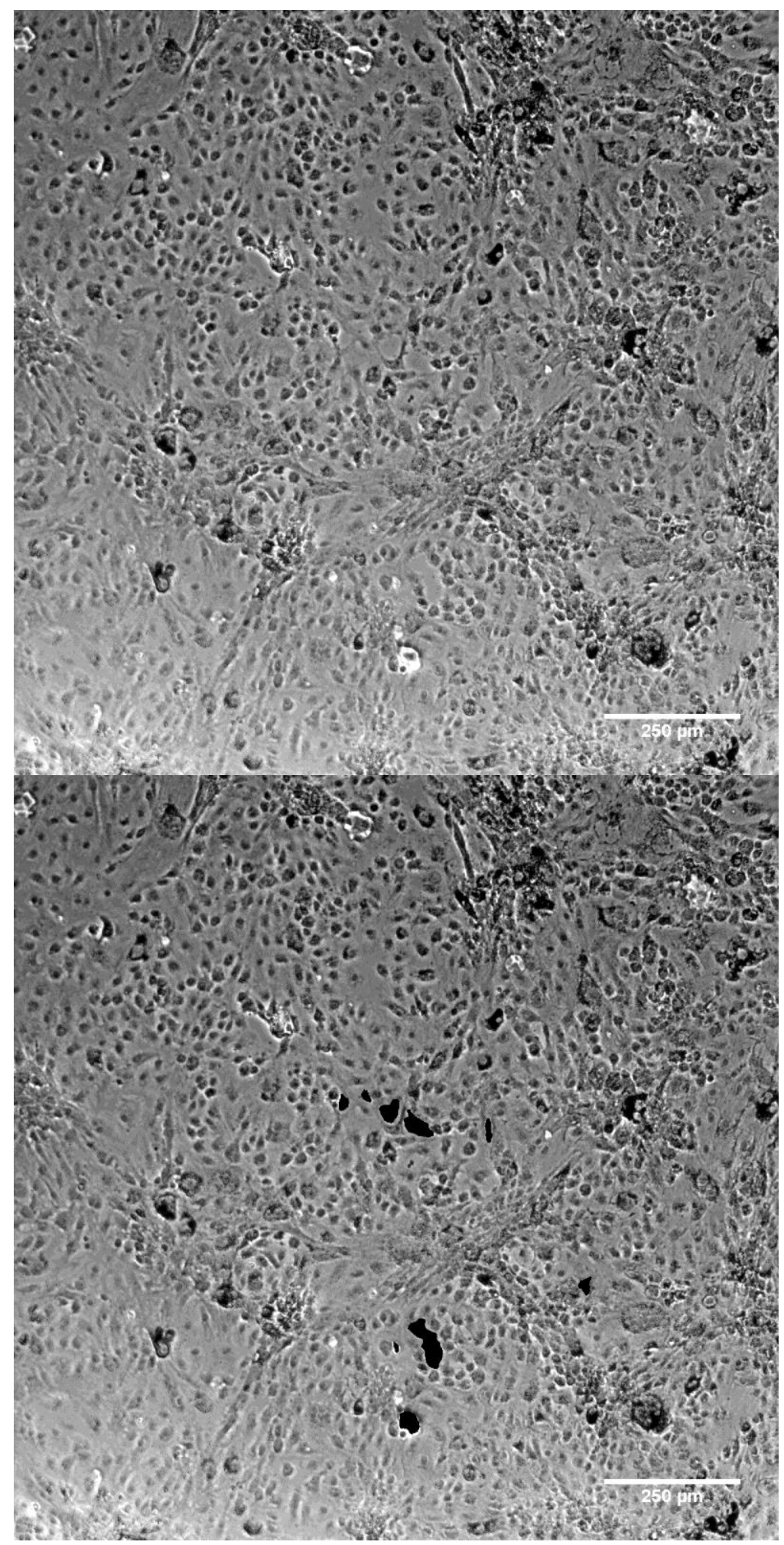

FIGURE A2.71- PRPE 10-3-2 Confluency 


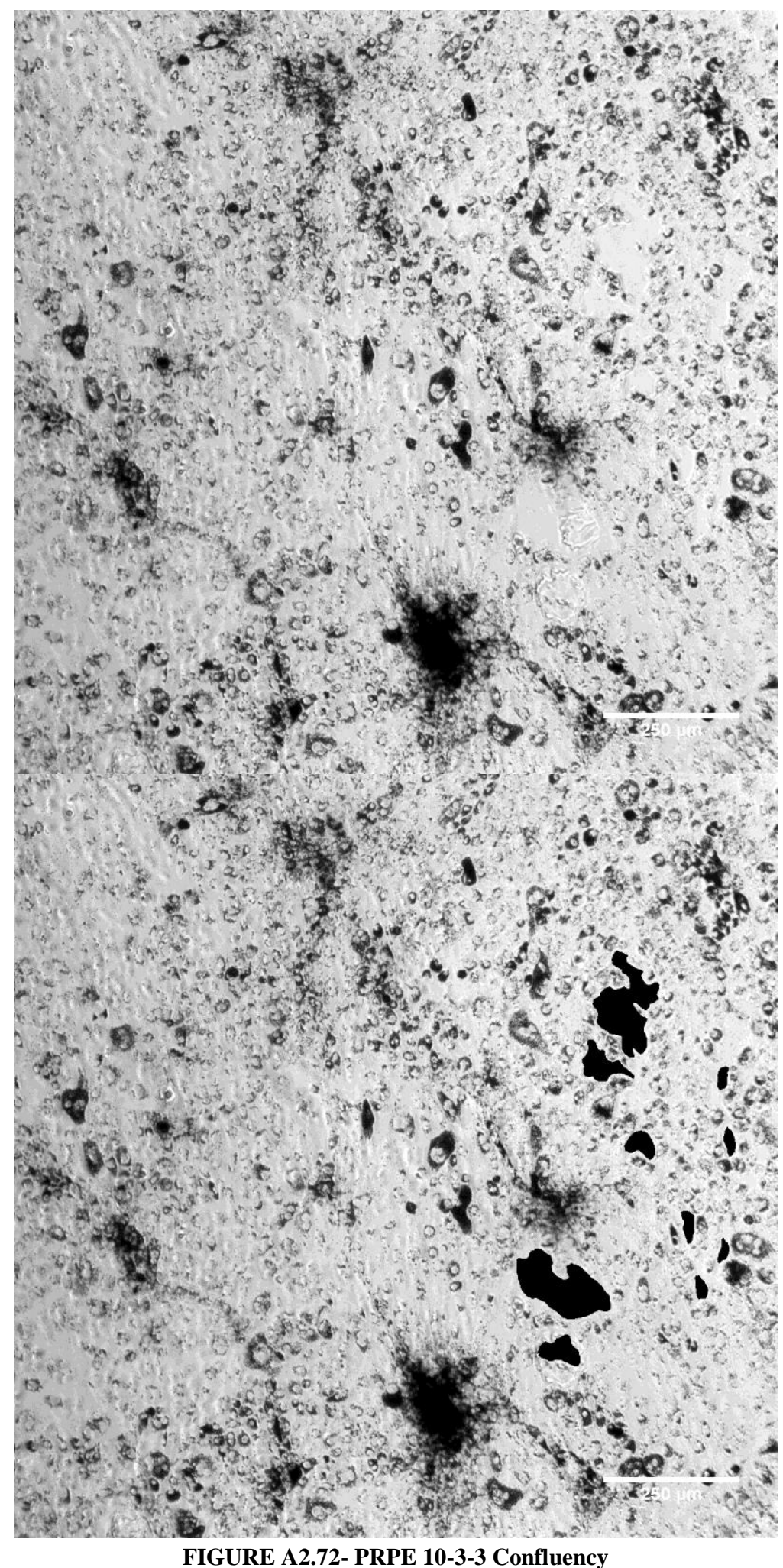




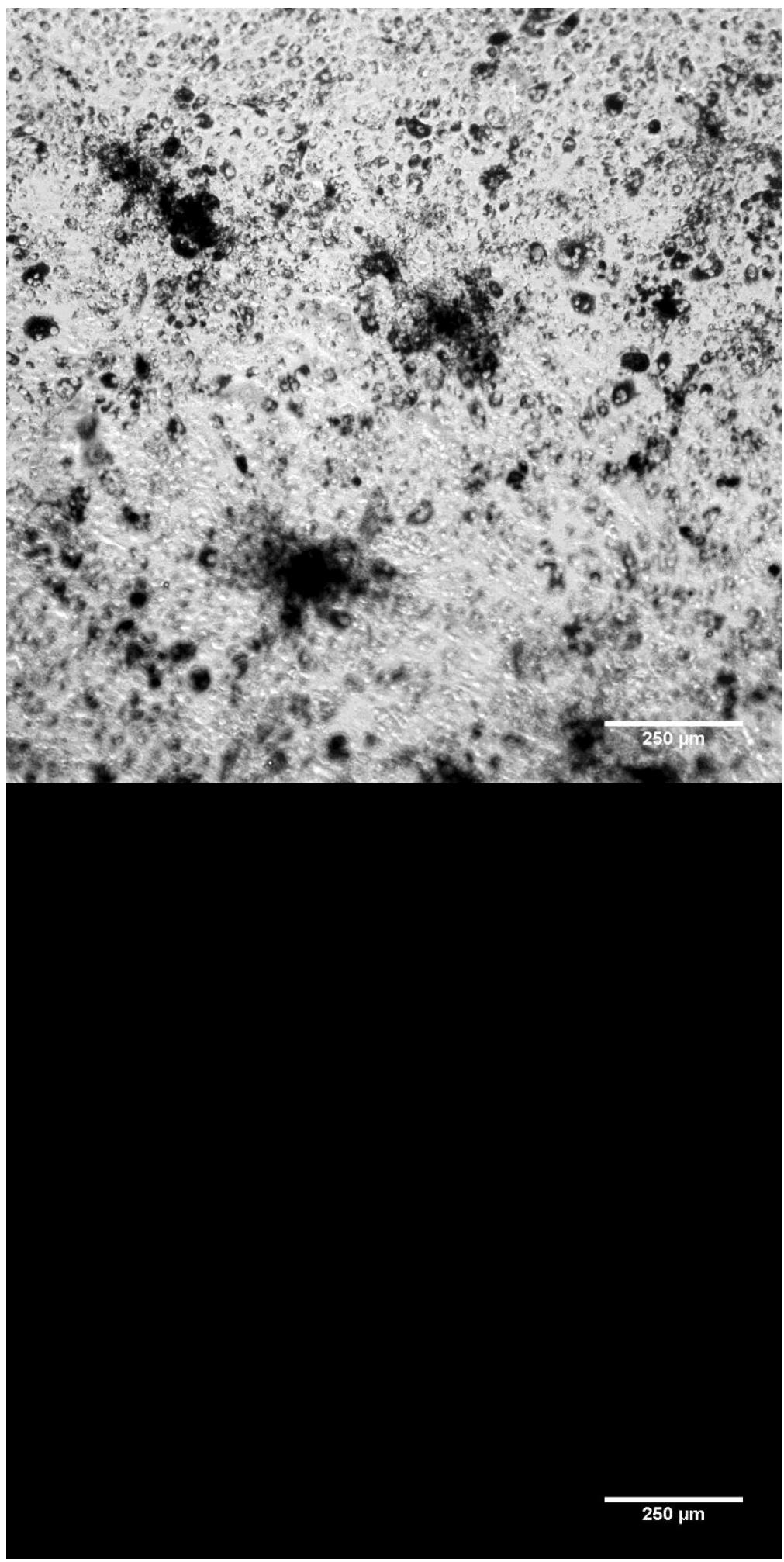

FIGURE A2.73- PRPE 20-1-1 Confluency 


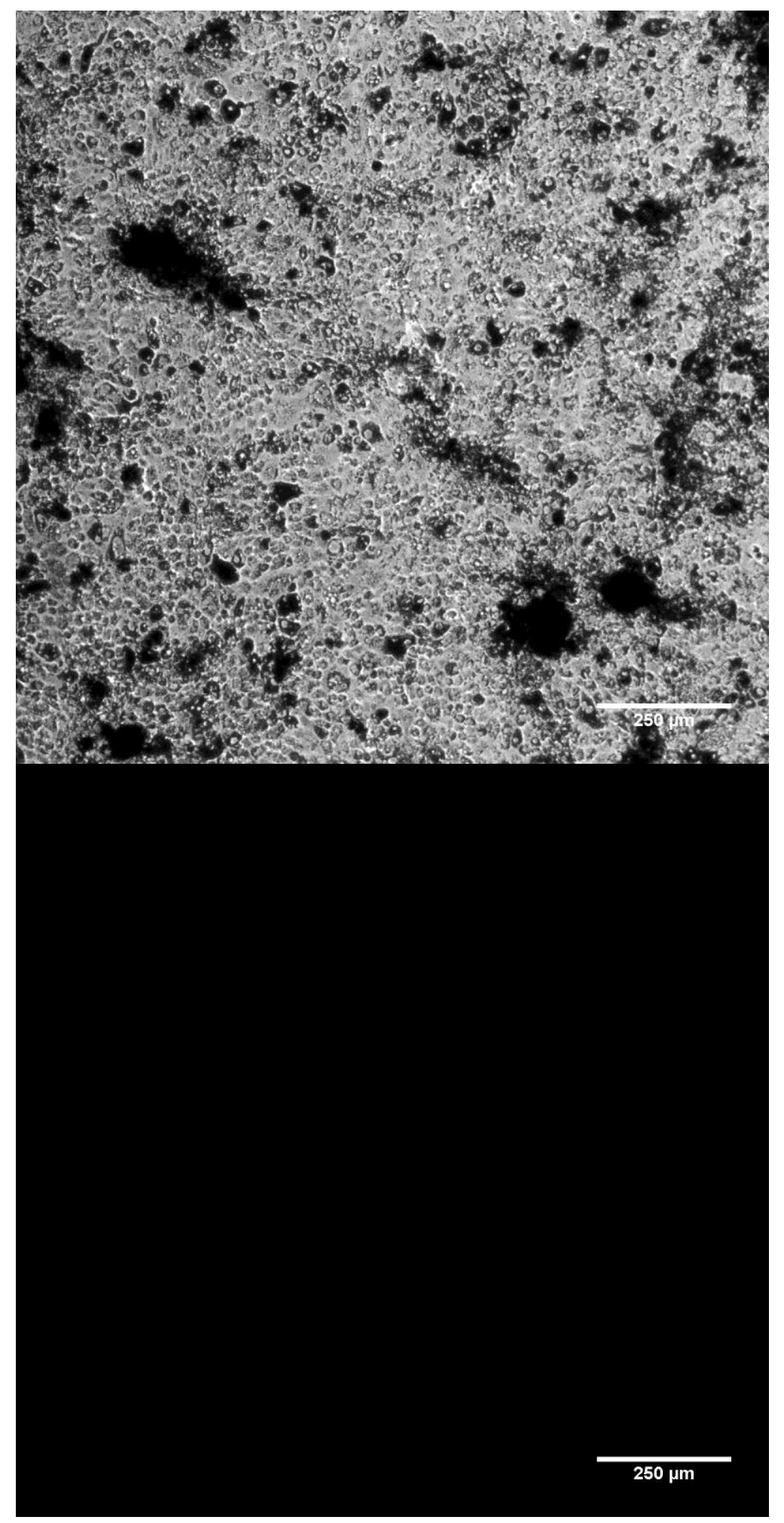

FIGURE A2.74- PRPE 20-1-2 Confluency 


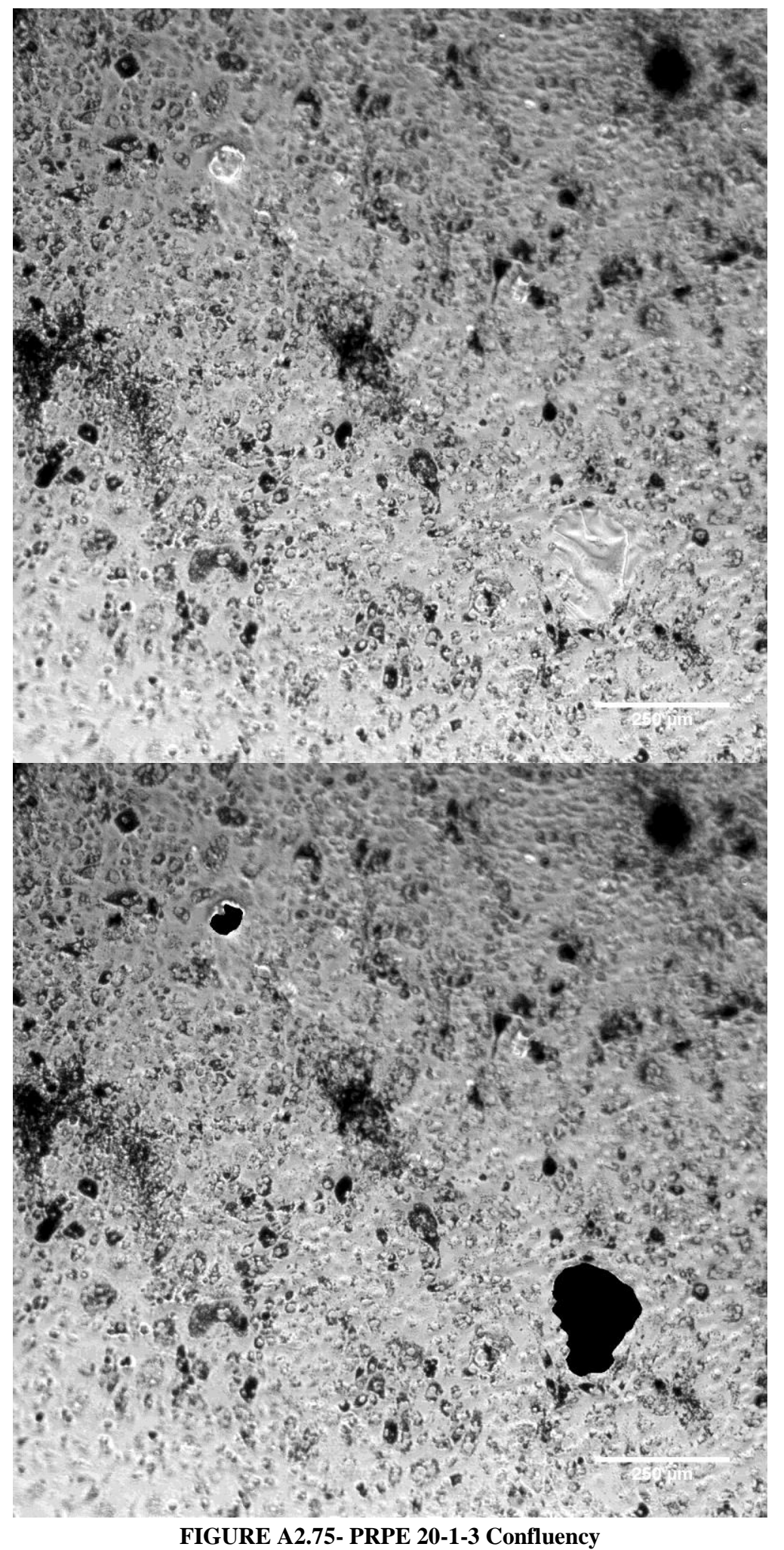




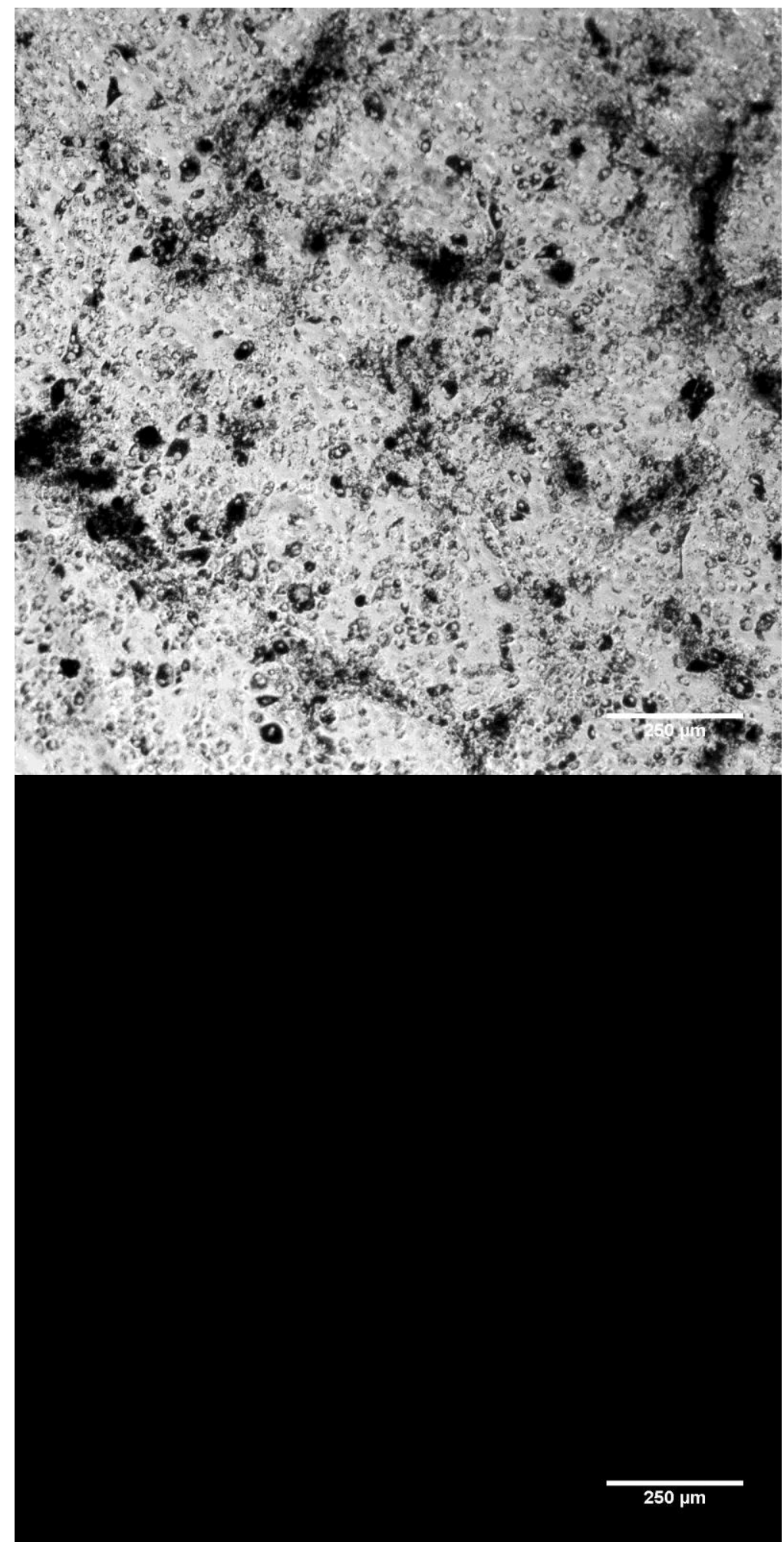

FIGURE A2.76- PRPE 20-2-1 Confluency 


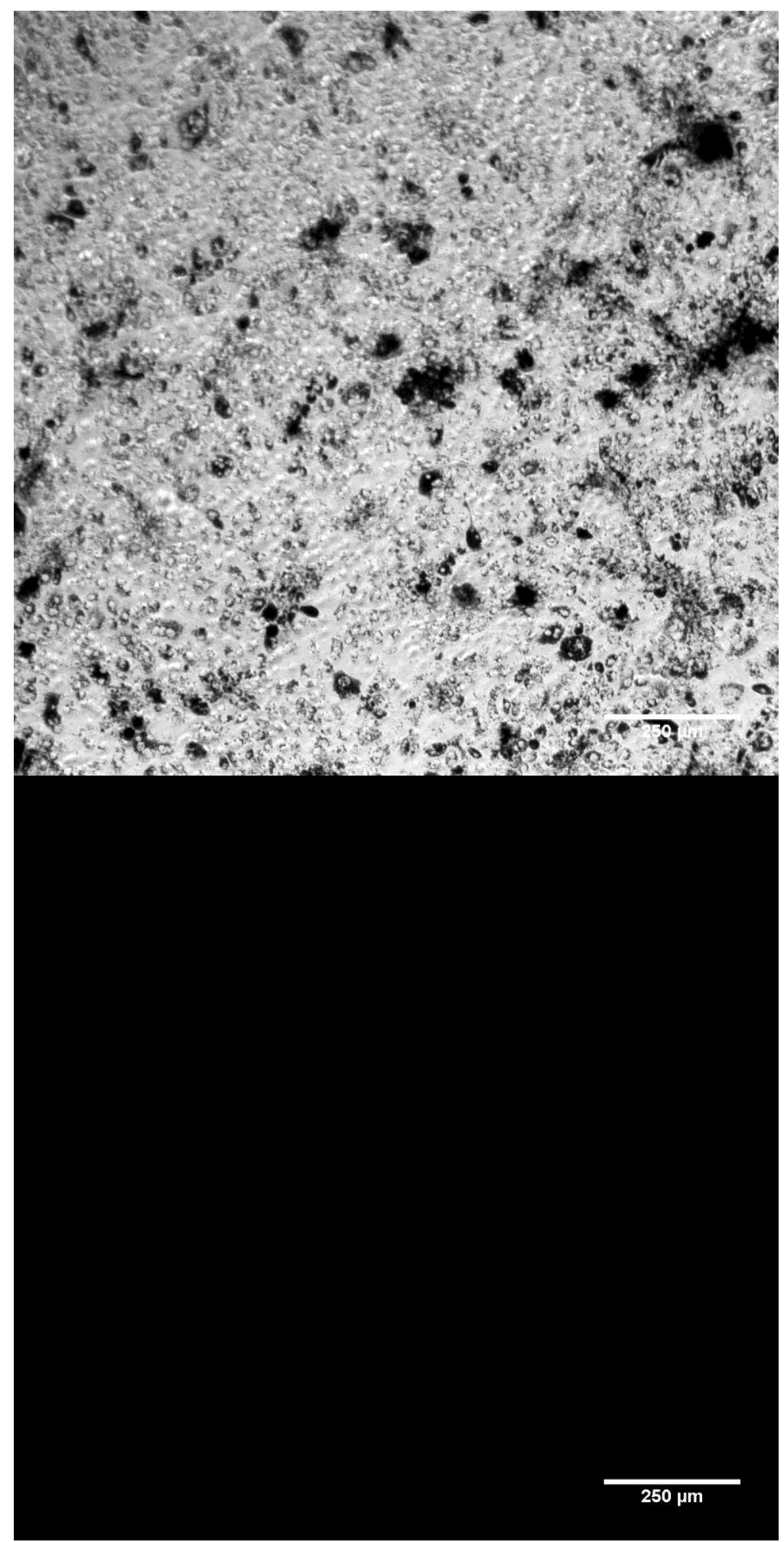

FIGURE A2.77- PRPE 20-2-2 Confluency 


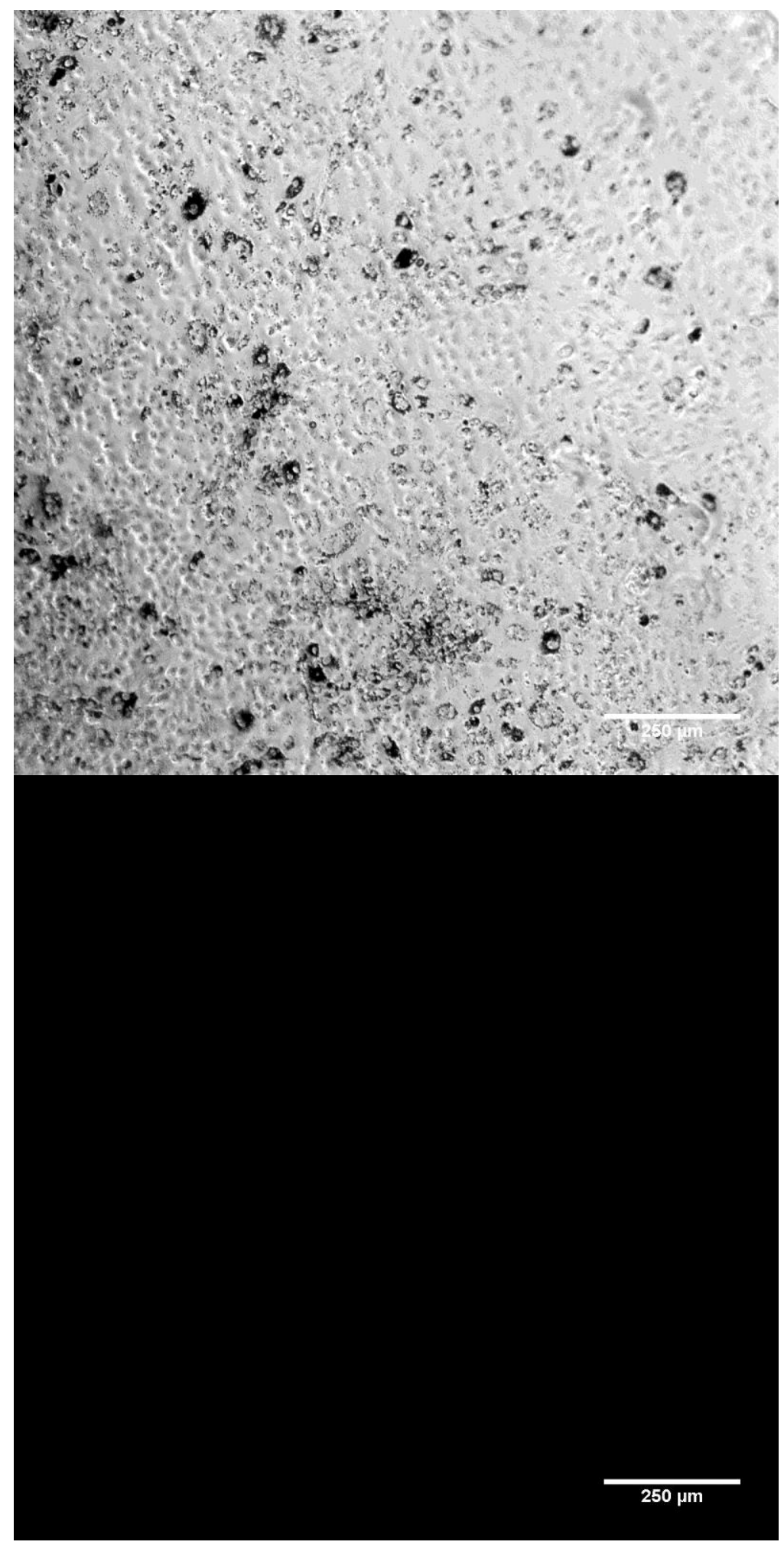

FIGURE A2.78- PRPE 20-2-3 Confluency 


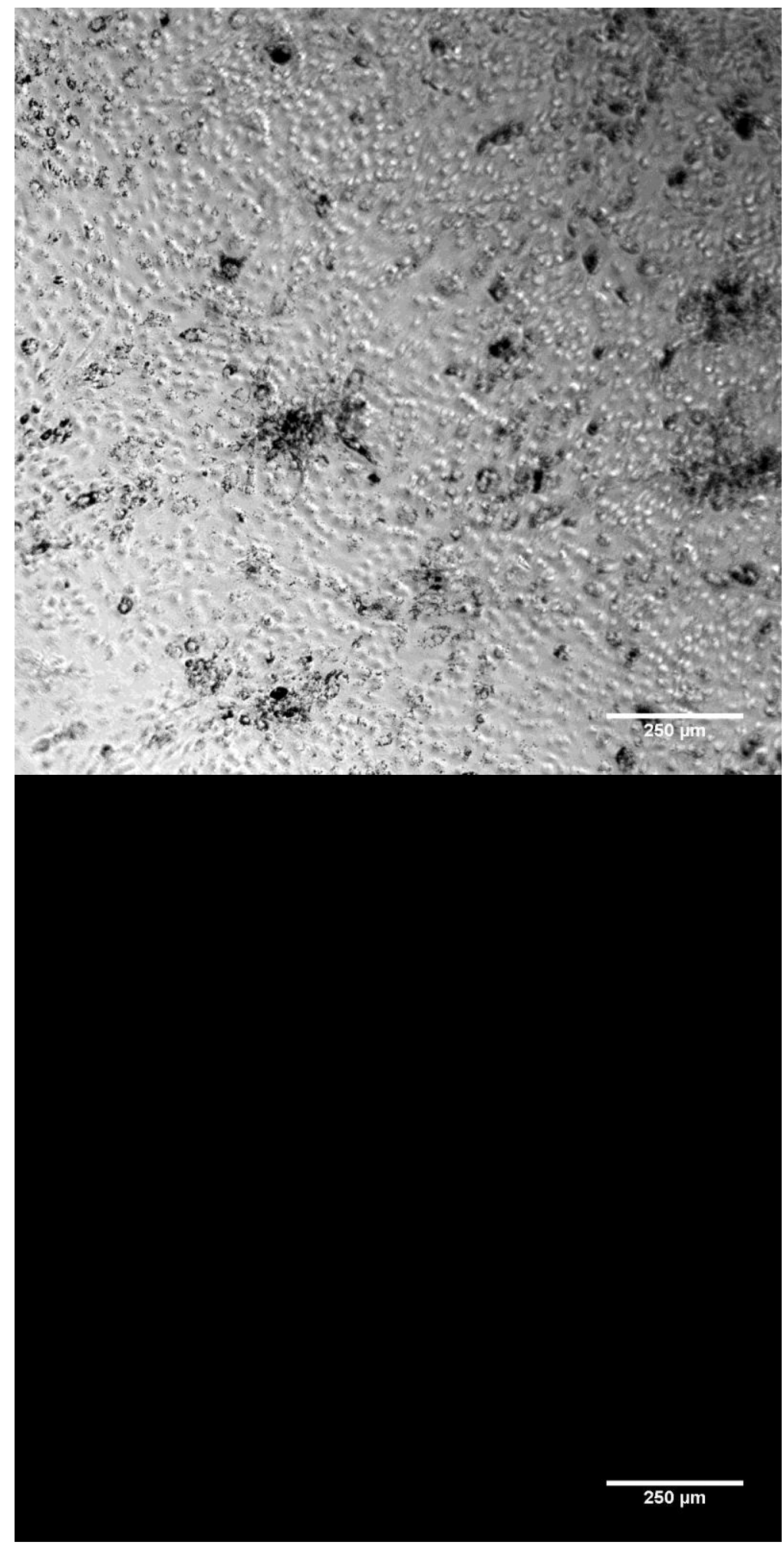

FIGURE A2.79- PRPE 20-3-1 Confluency 


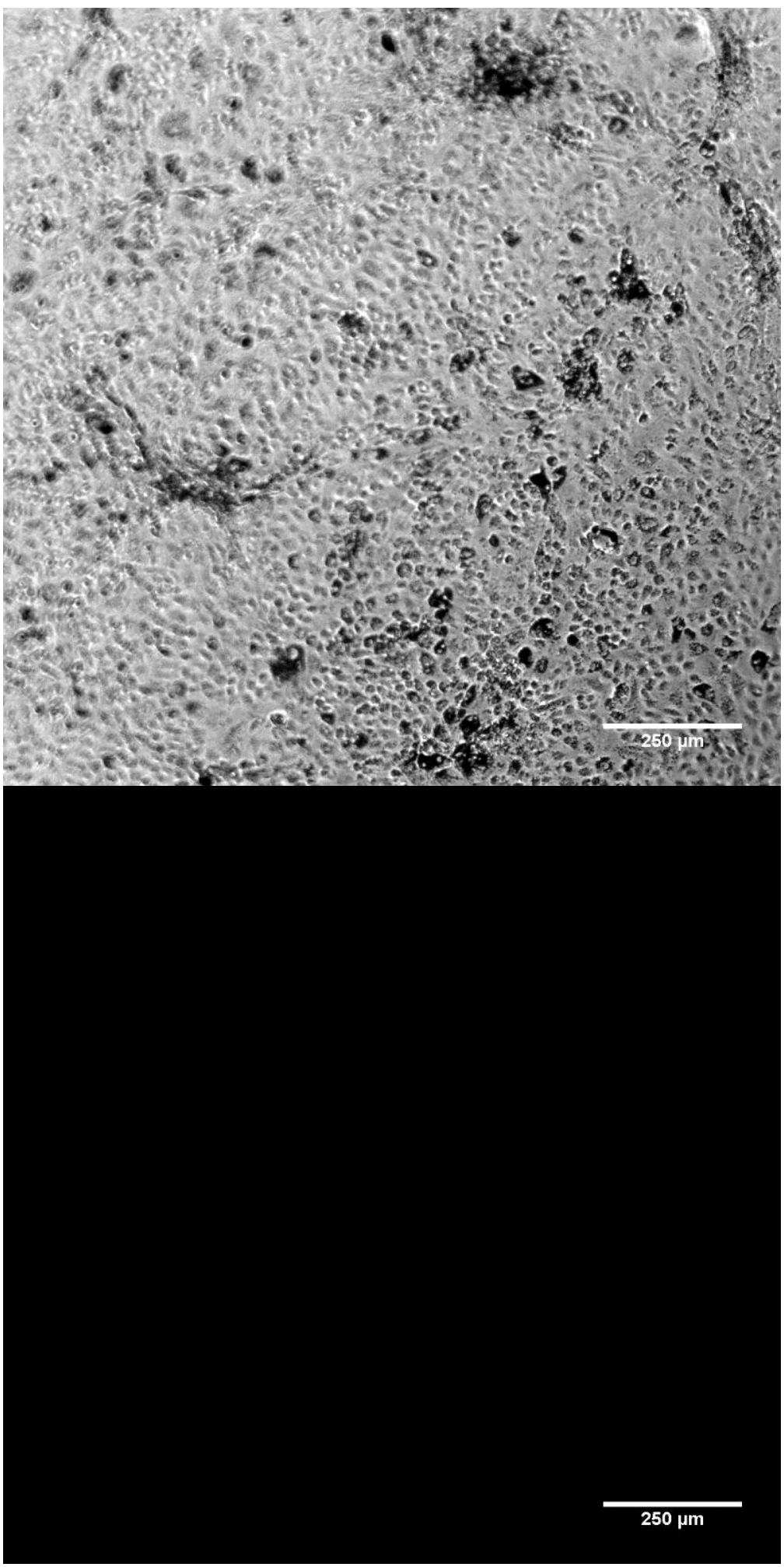

FIGURE A2.80- PRPE 20-3-2 Confluency 


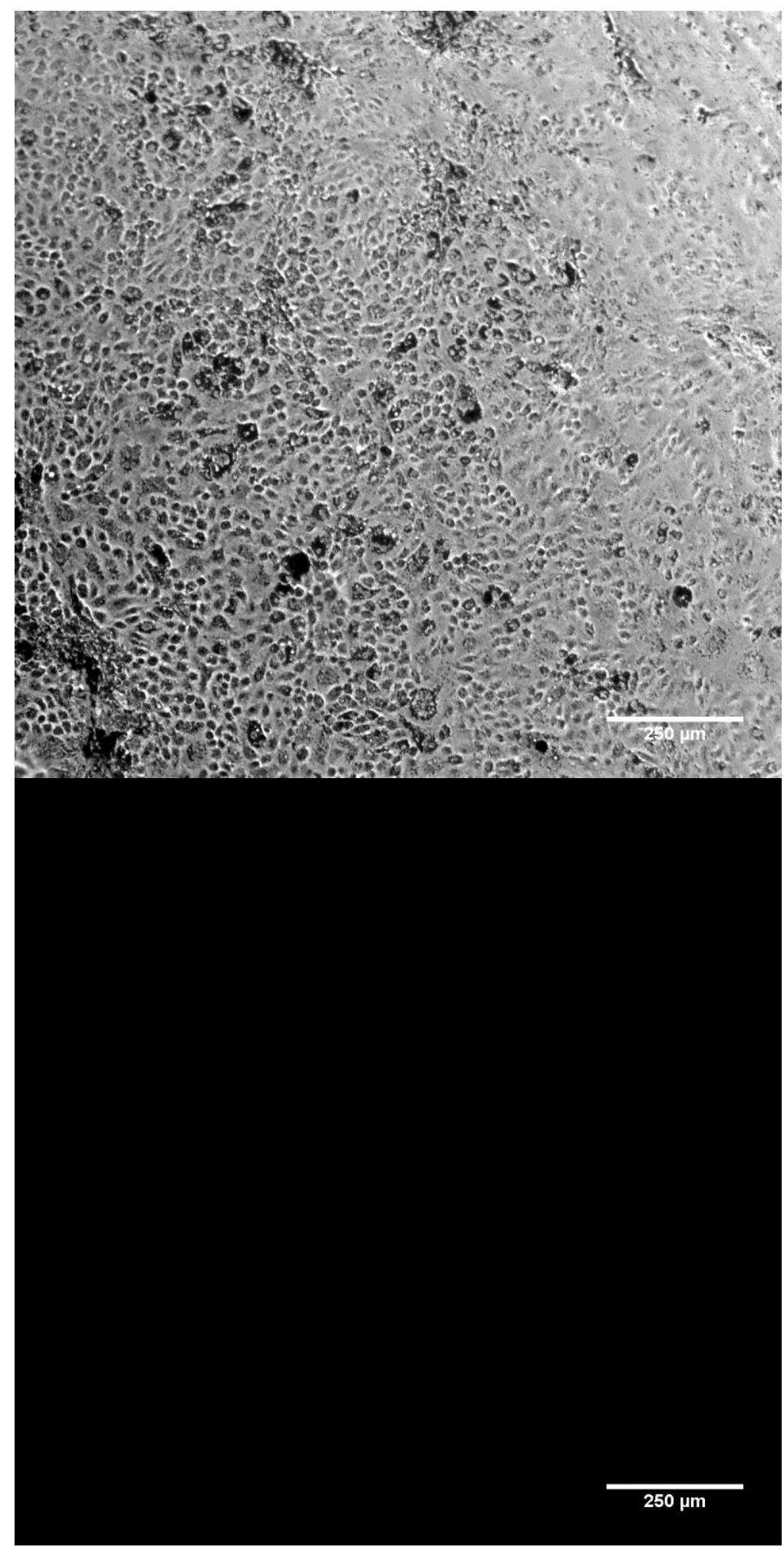

FIGURE A2.81- PRPE 20-3-3 Confluency 


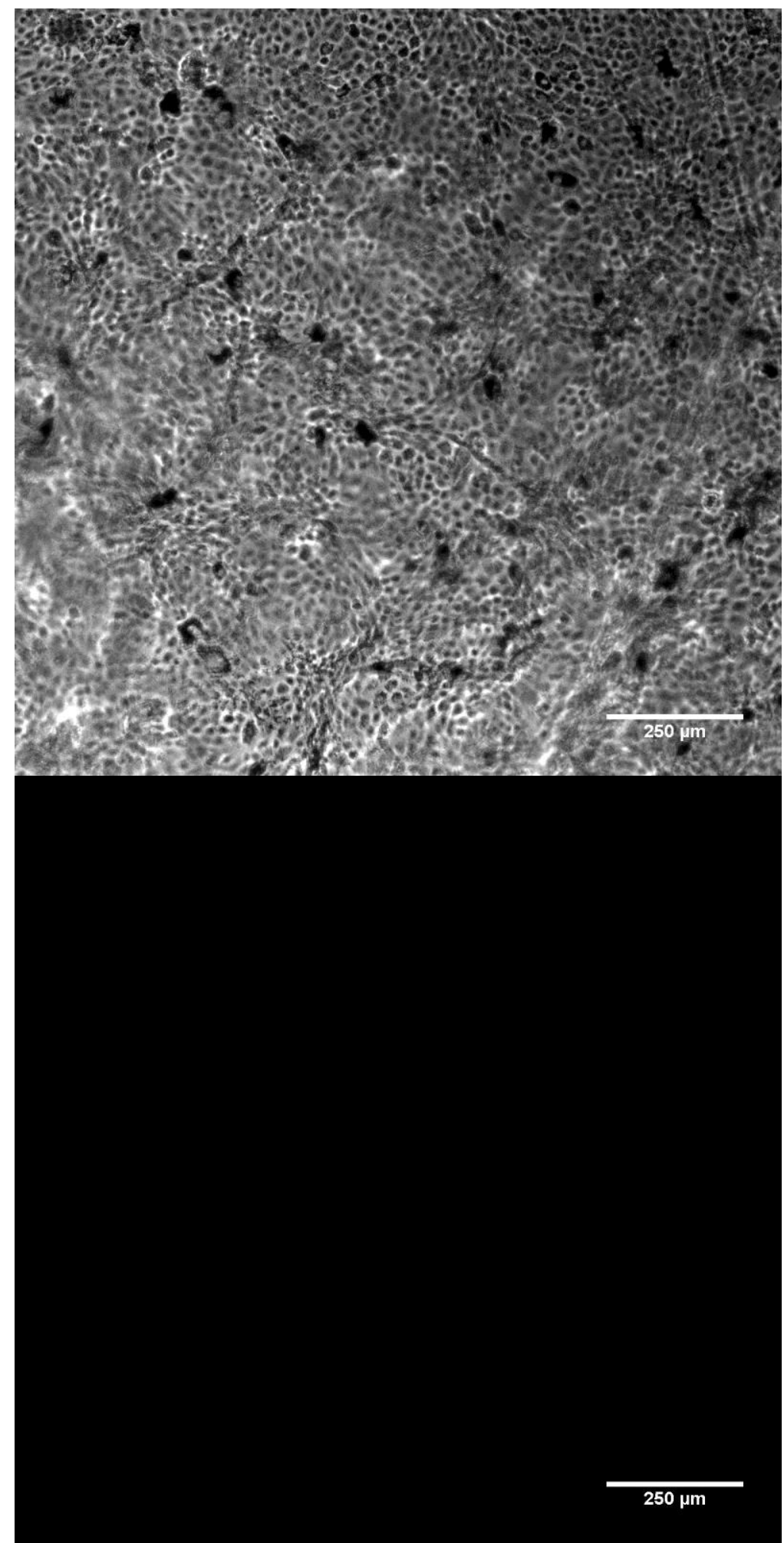

FIGURE A2.82- PRPE FN-1-1 Confluency 


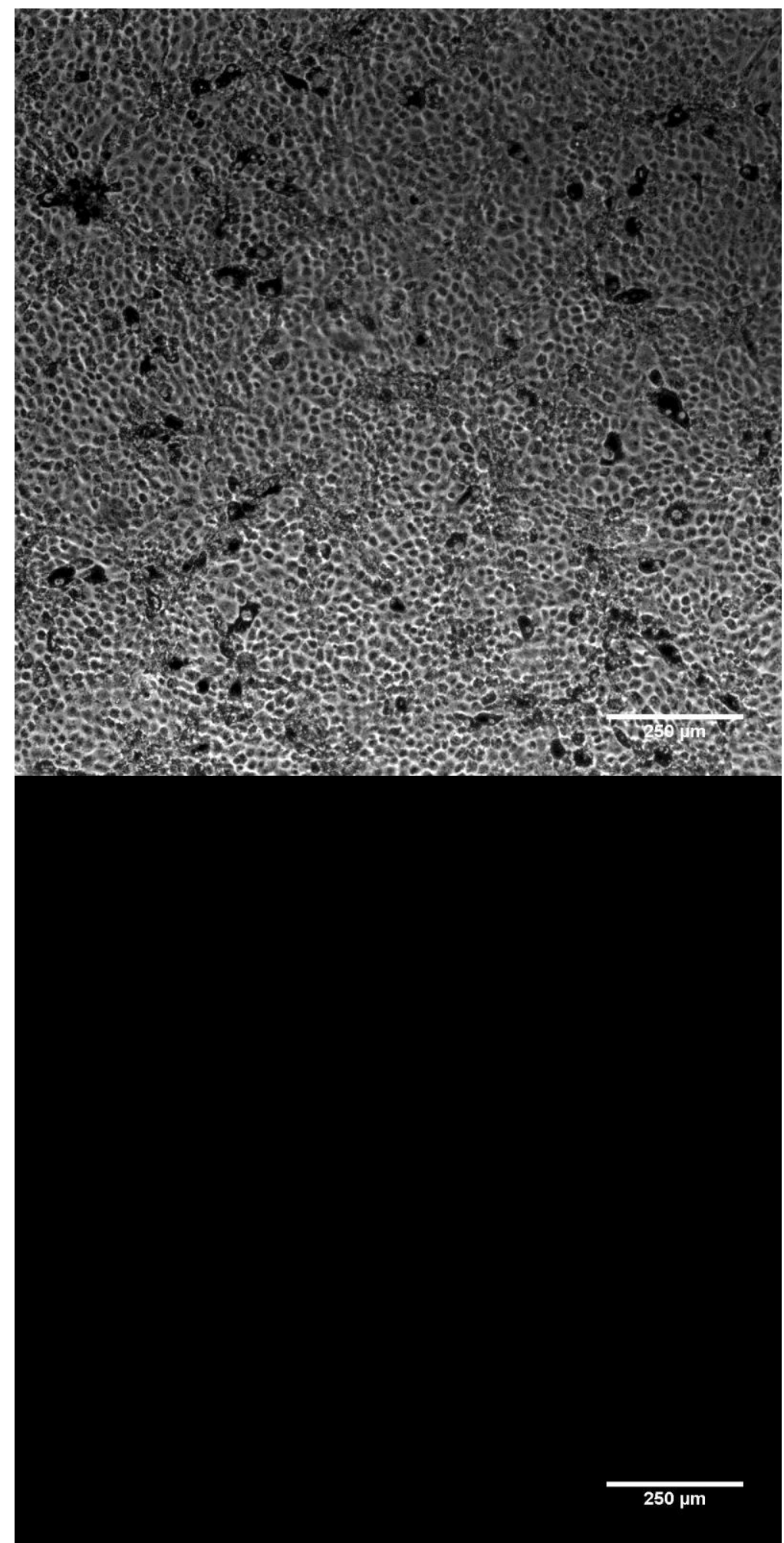

FIGURE A2.83- PRPE FN-1-2 Confluency 


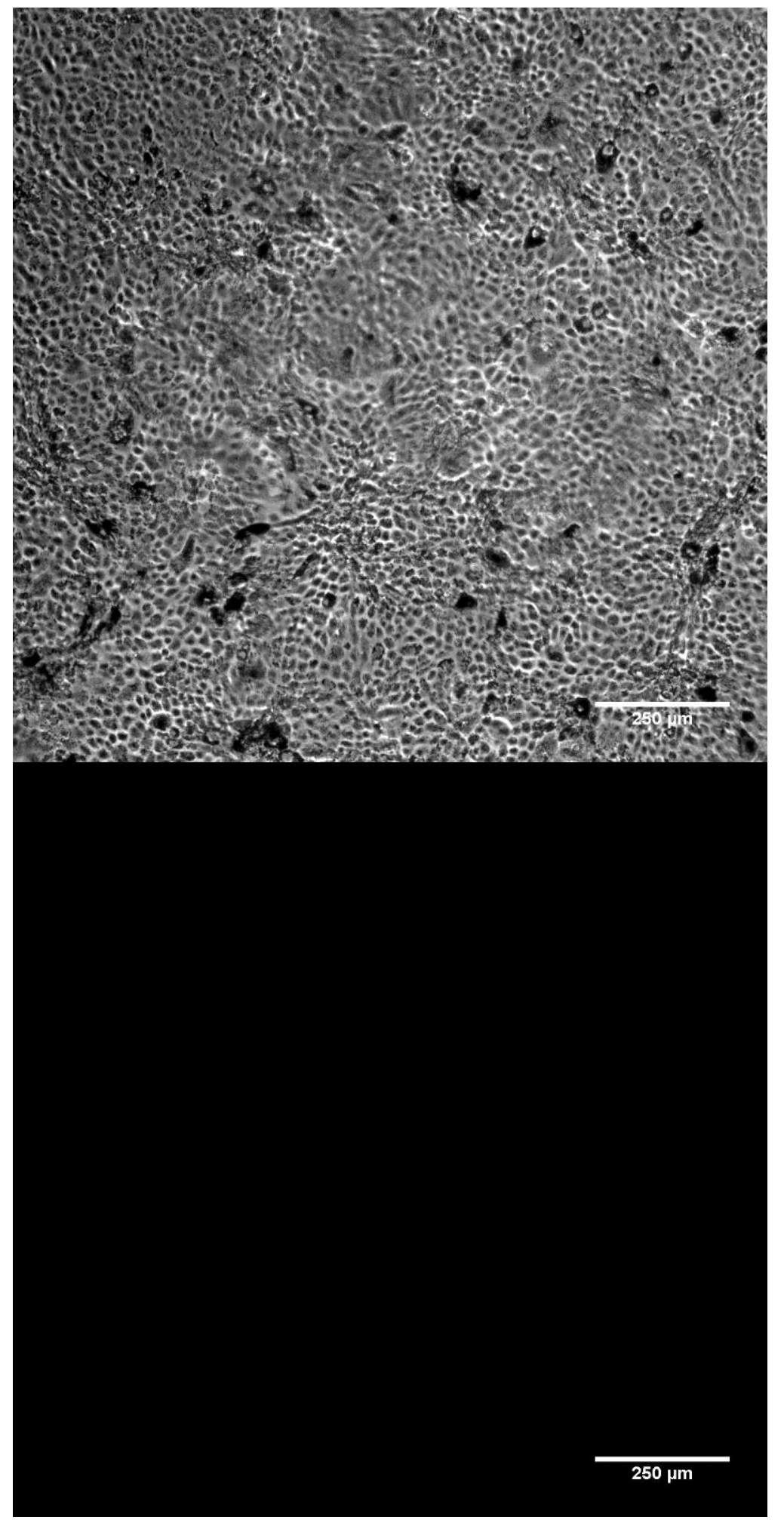

FIGURE A2.84- PRPE FN-1-3 Confluency 


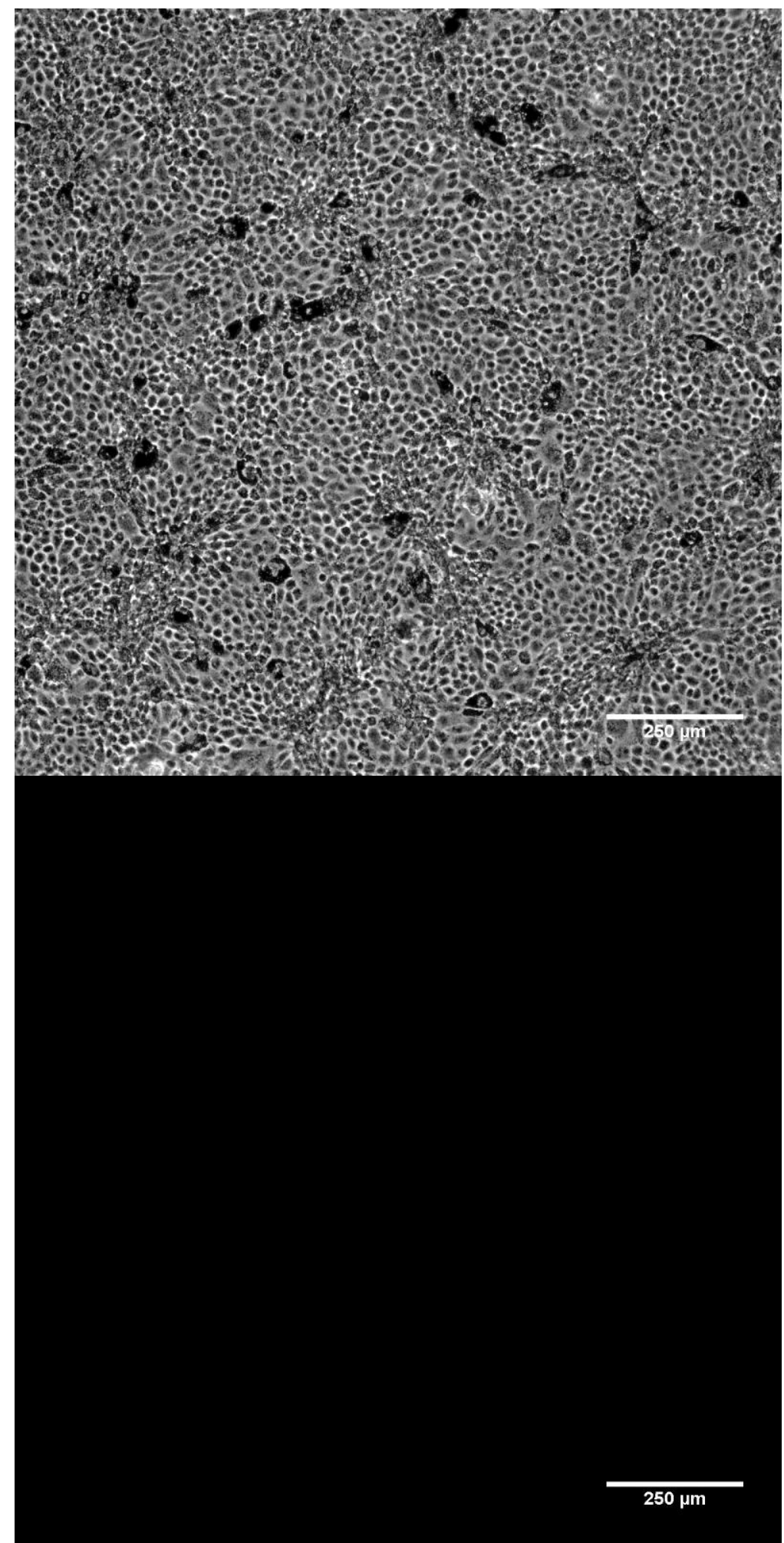

FIGURE A2.85- PRPE FN-2-1 Confluency 


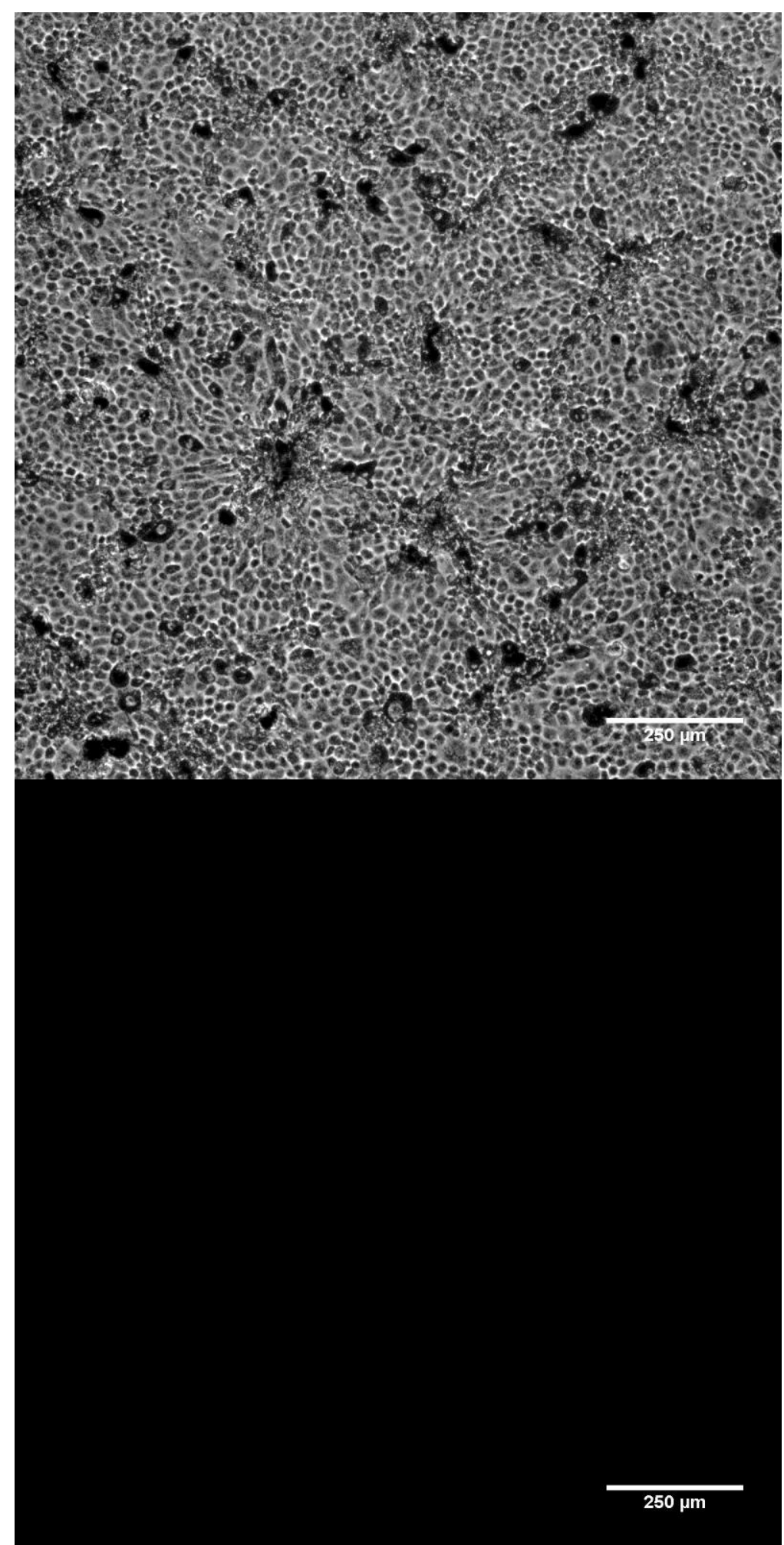

FIGURE A2.86- PRPE FN-2-2 Confluency 


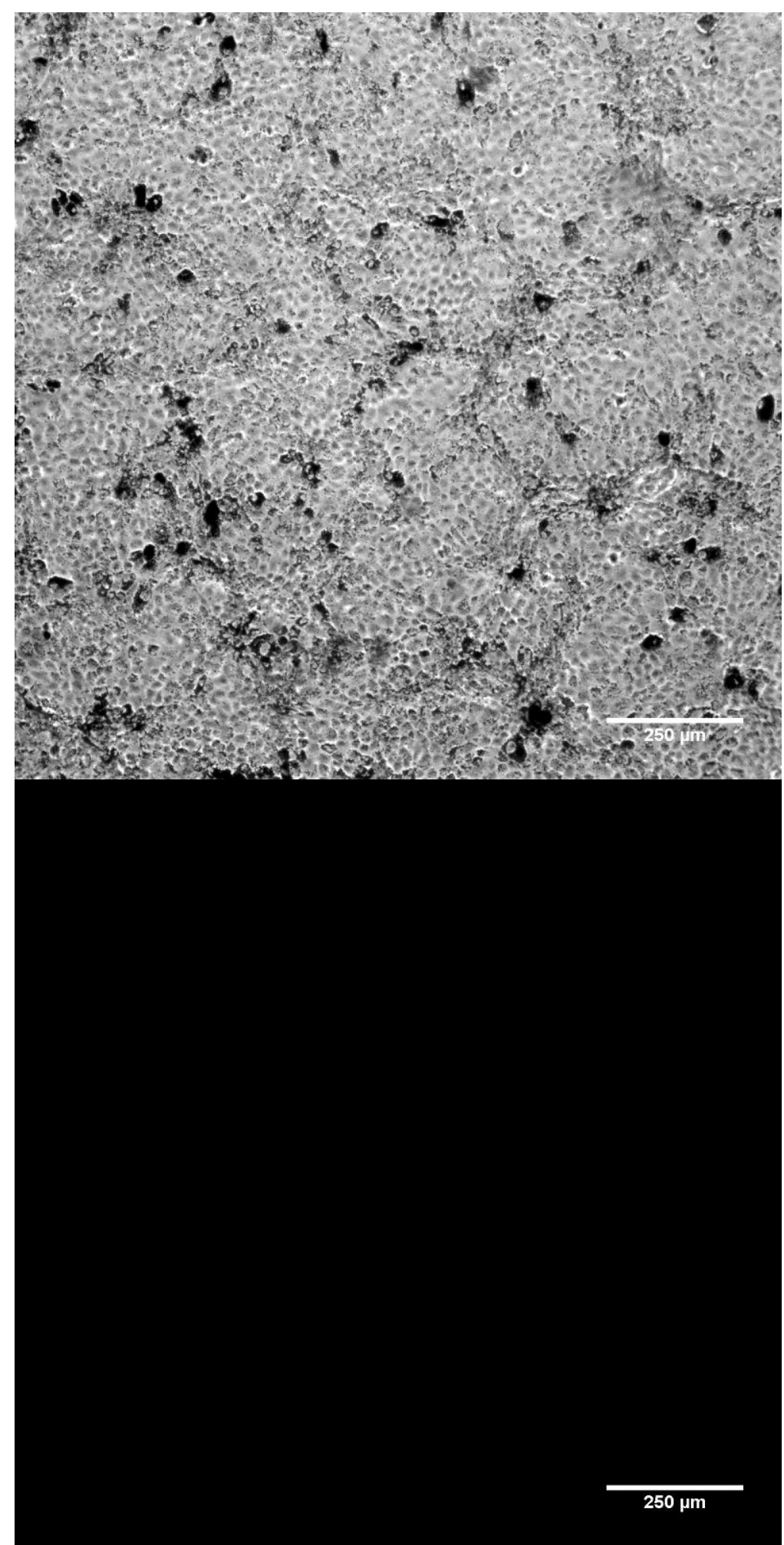

FIGURE A2.87- PRPE FN-2-3 Confluency 


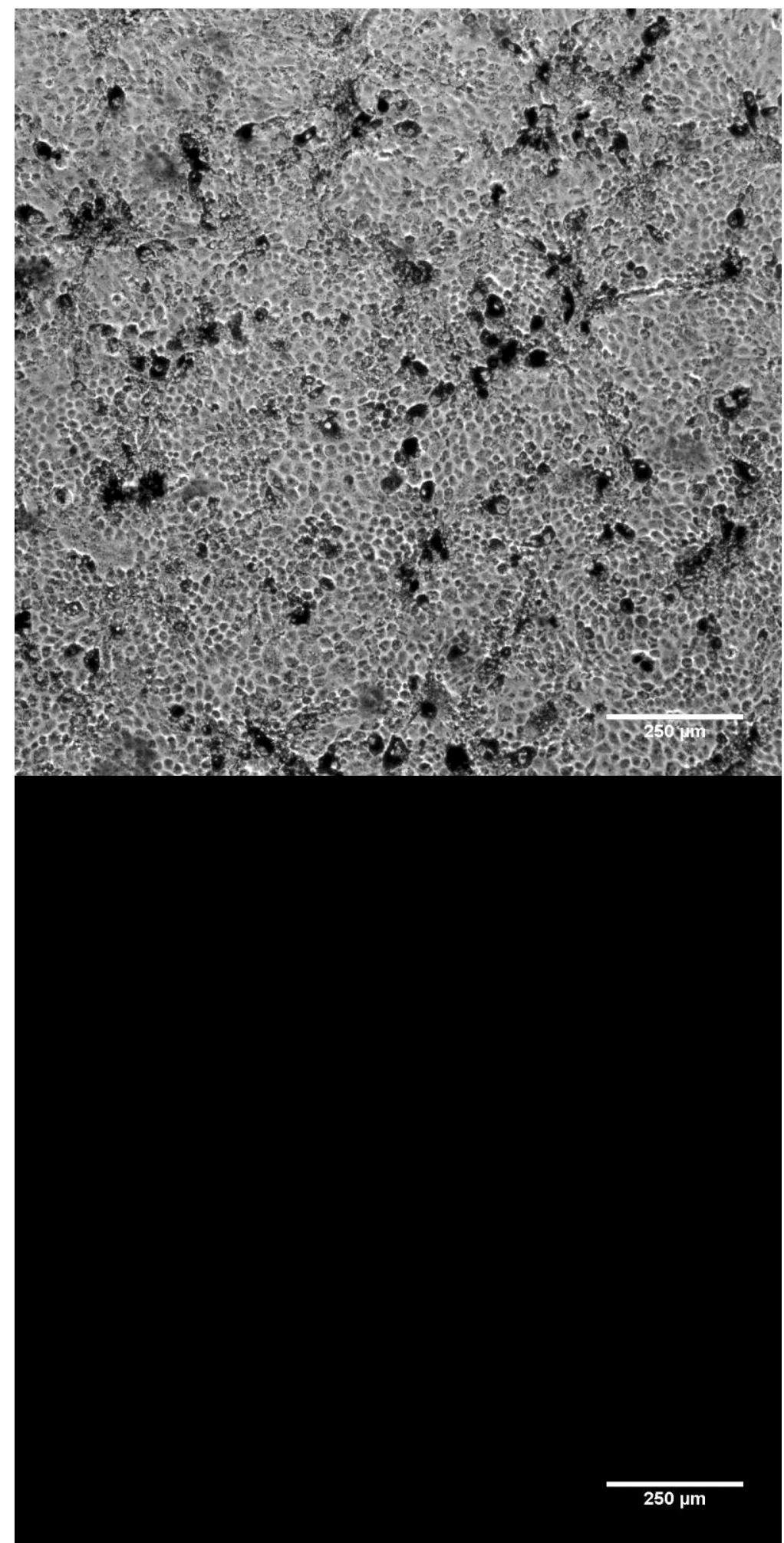

FIGURE A2.88- PRPE FN-3-1 Confluency 


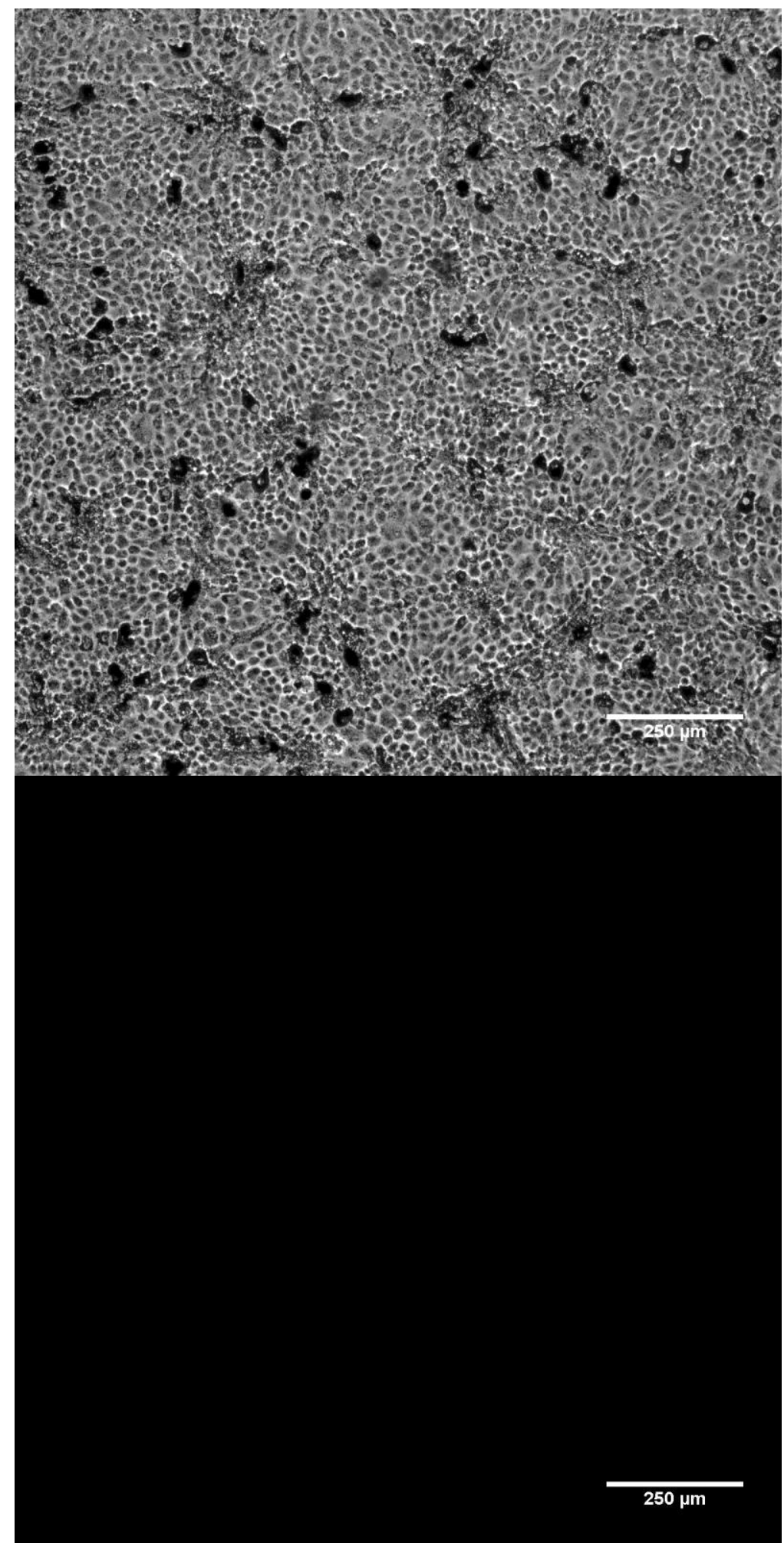

FIGURE A2.89- PRPE FN-3-2 Confluency 


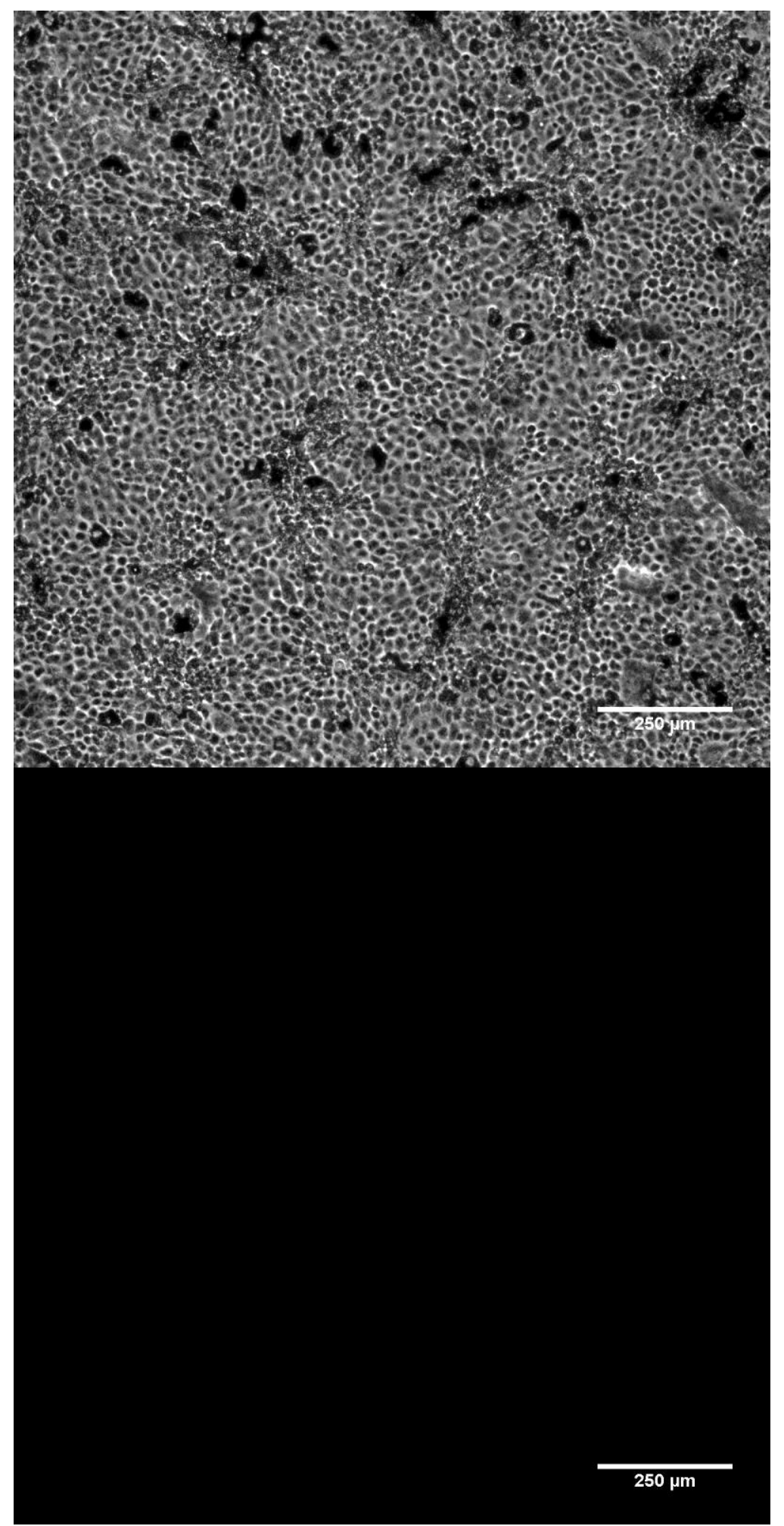

FIGURE A2.90- PRPE FN-3-3 Confluency 


\title{
APPENDIX III: Confocal Microscopy Images
}

\author{
All images follow the same naming format: \\ (Cell Type) -(Concentration)-(Gel Number)-(Image Number) (Date Taken) \\ All images are taken at 60x. \\ Top left: Composite image \\ Top right: Actin Staining \\ Bottom Left: DAPI staining \\ Bottom Right: ZO-1 staining
}








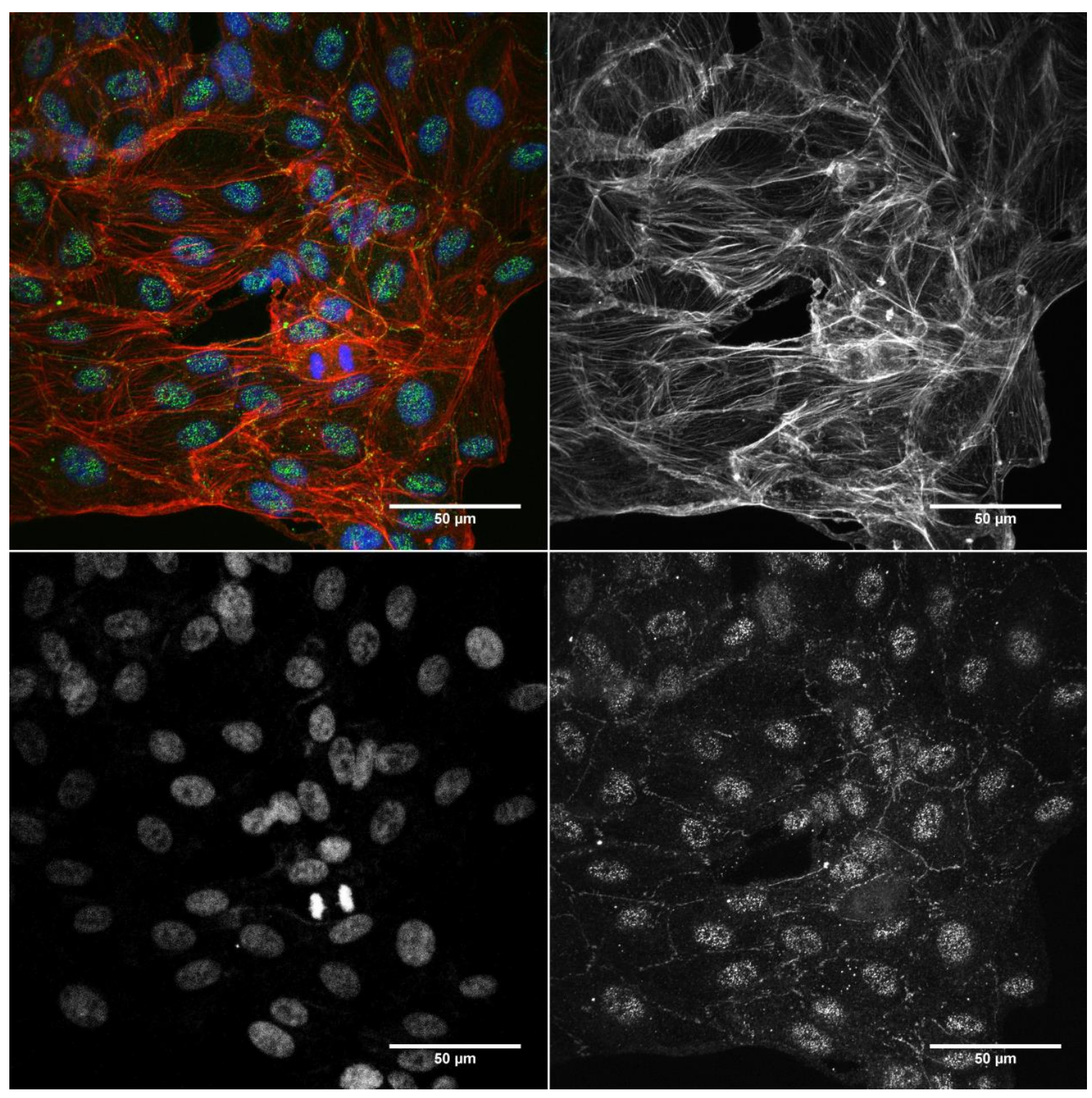

FIGURE A3.5- ARPE 5-1-1 (5/10/13) 


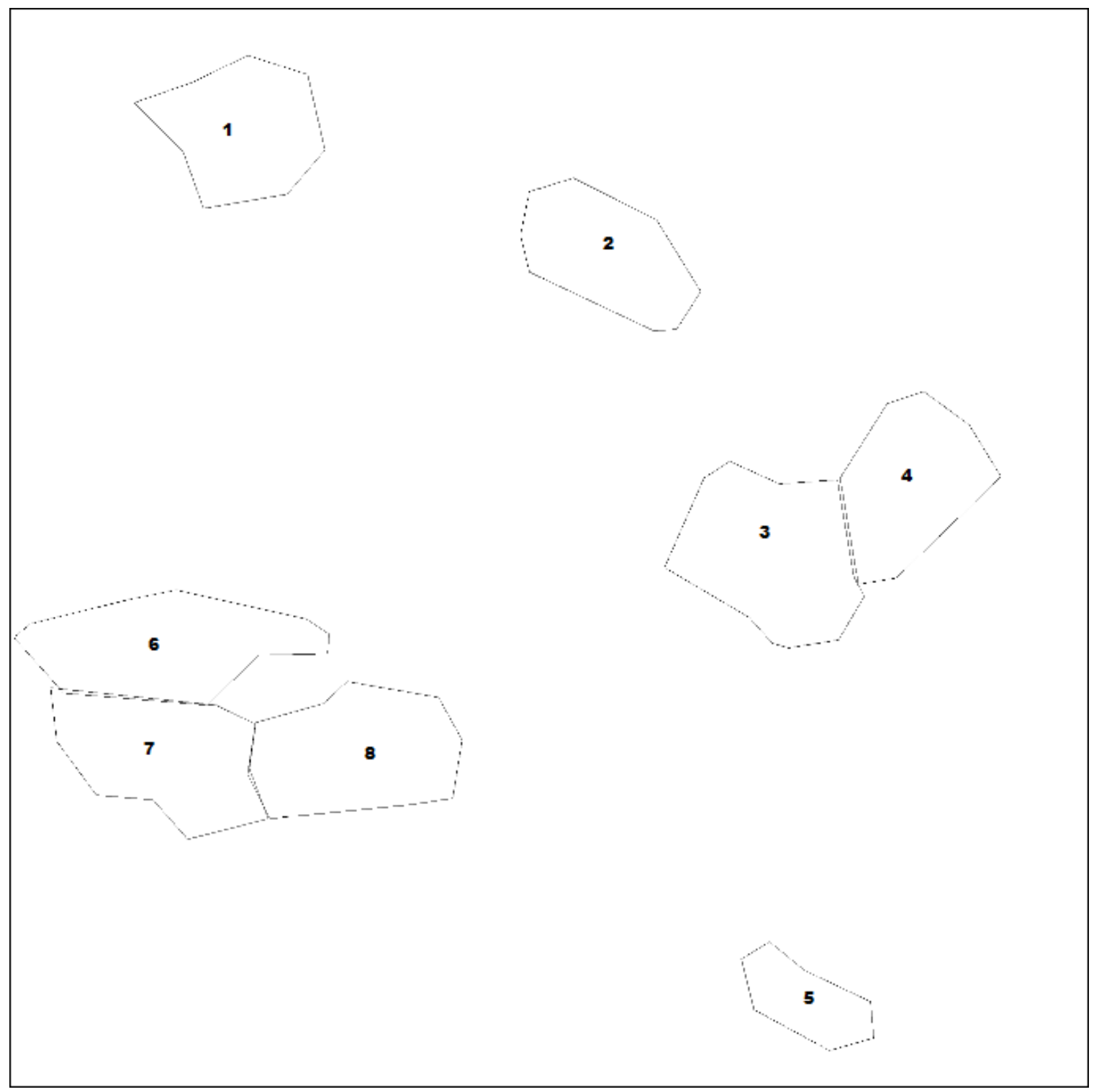

FIGURE A3.6- ARPE 5-1-1 ZO-1 Analysis 


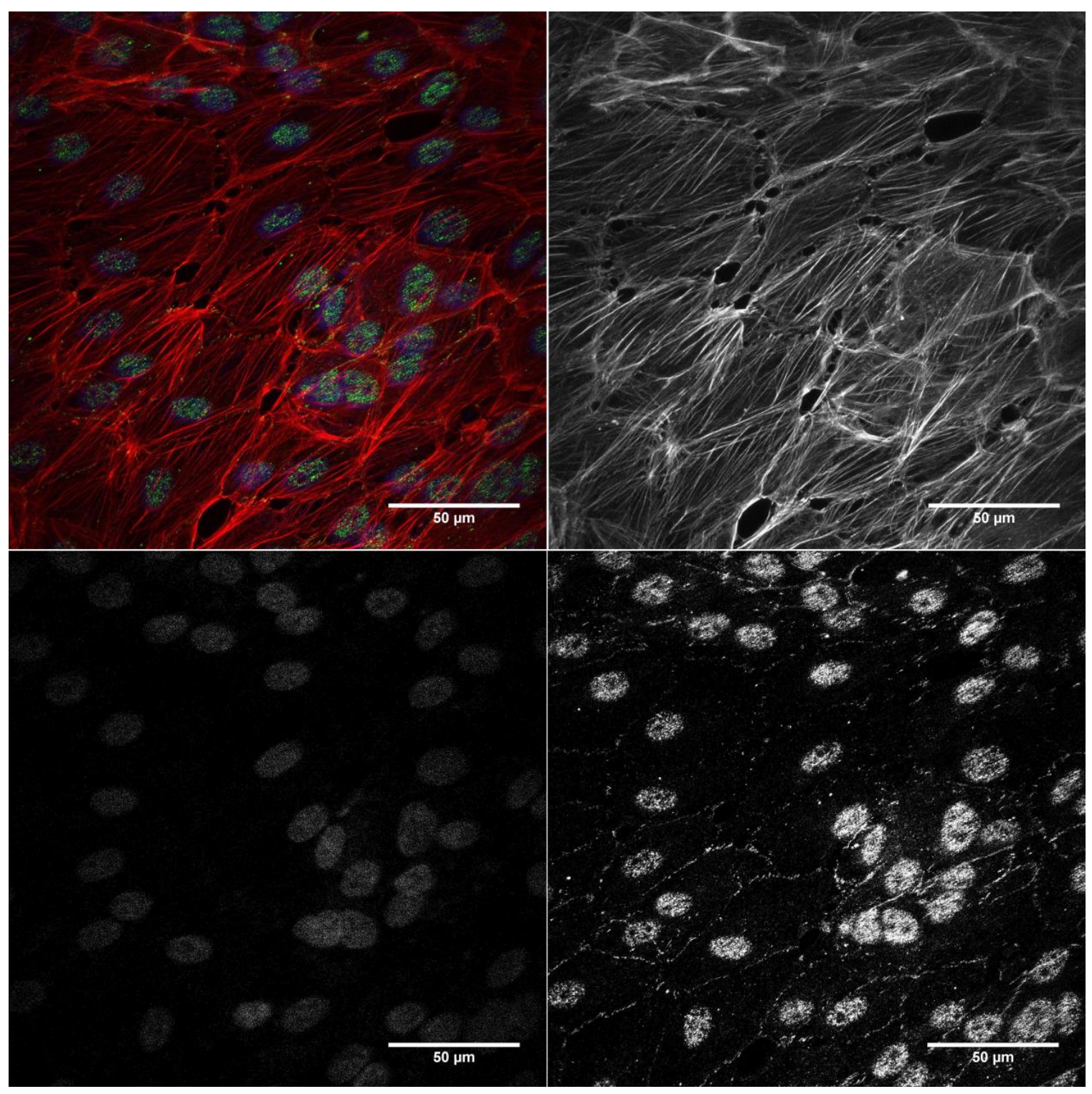

FIGURE A3.7- ARPE 10-1-1 (5/10/13) 


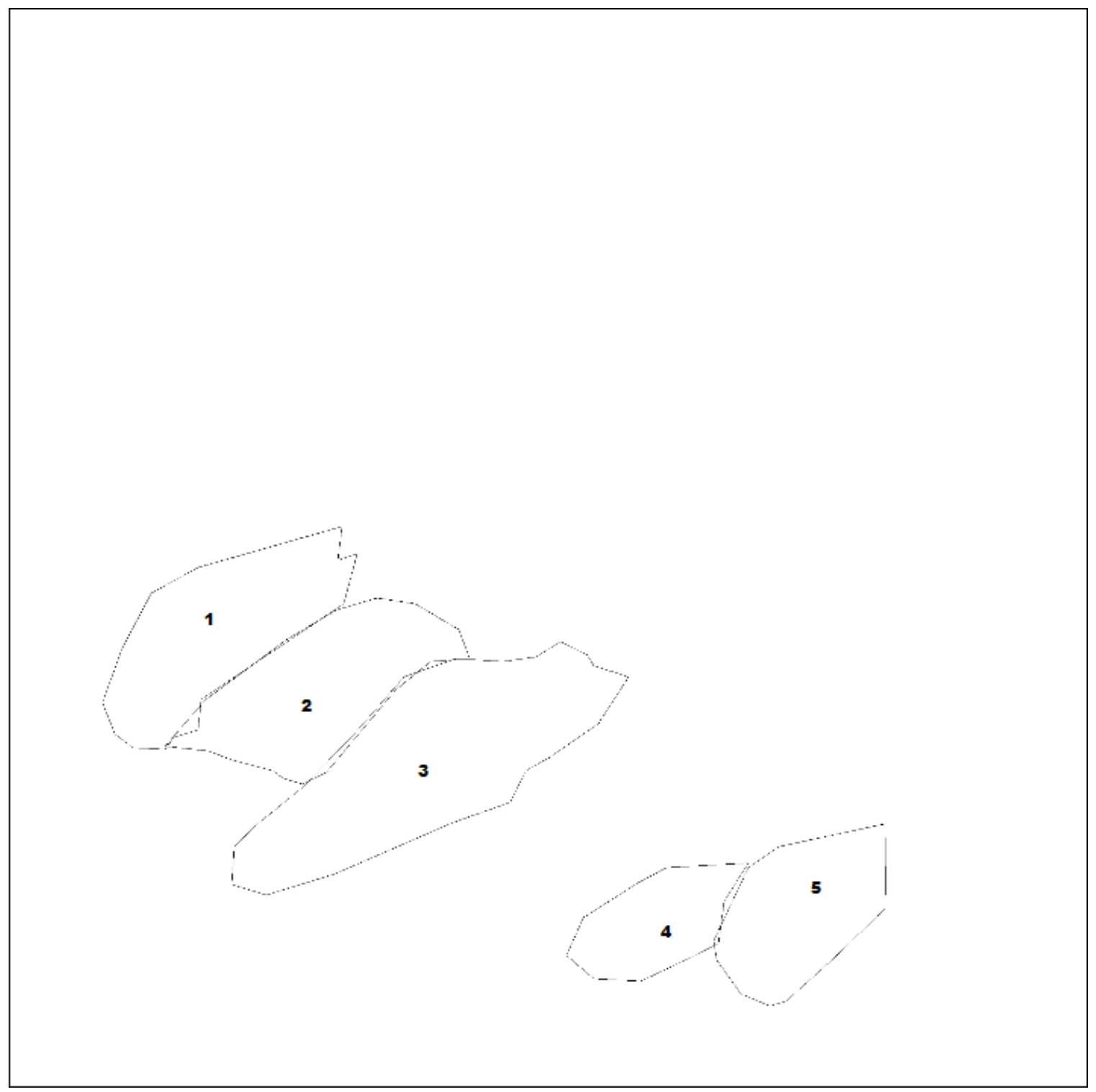

FIGURE A3.8- ARPE 10-1-1 ZO-1 Analysis 


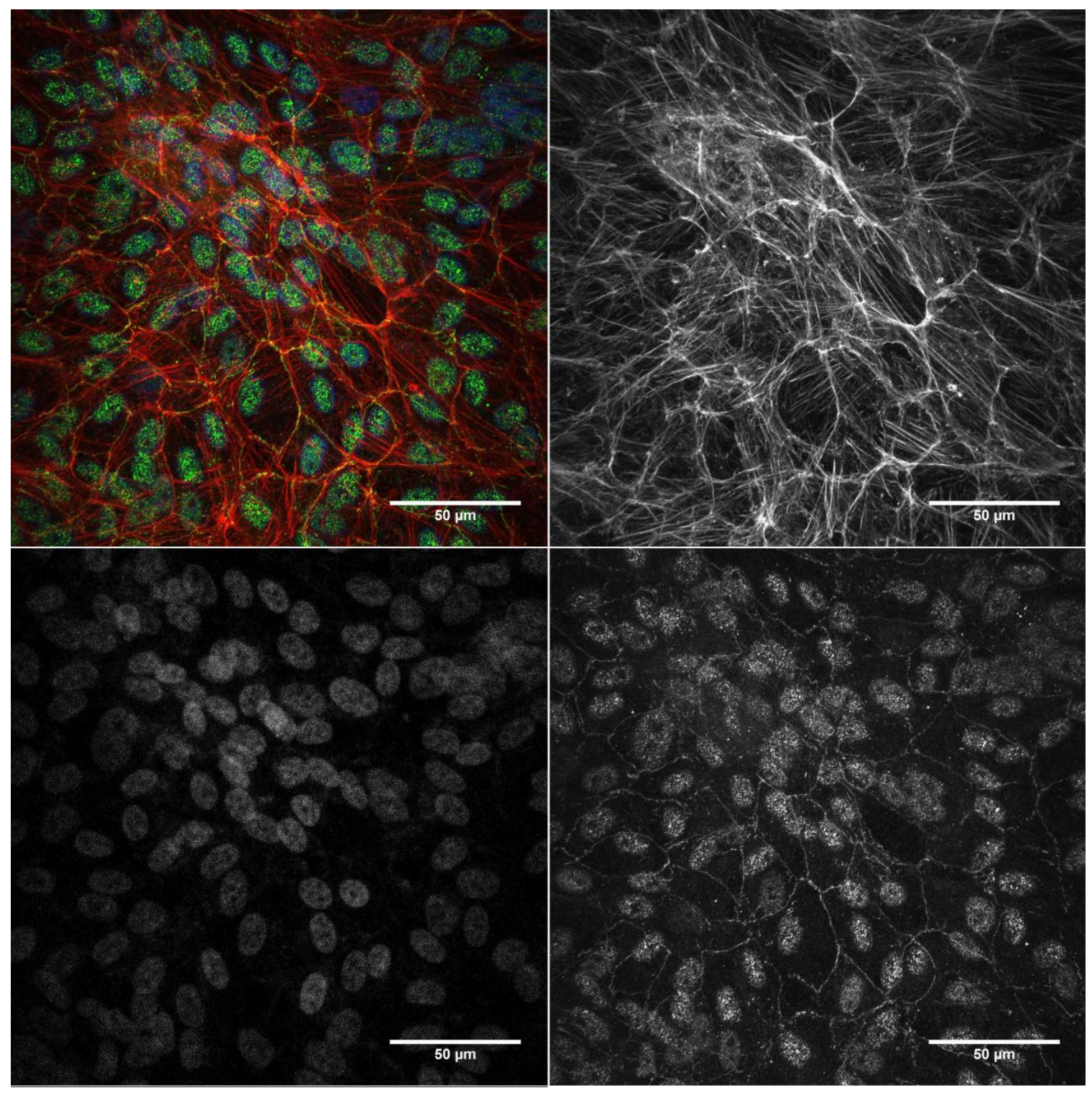

FIGURE A3.9- ARPE 10-2-1 (5/10/13) 


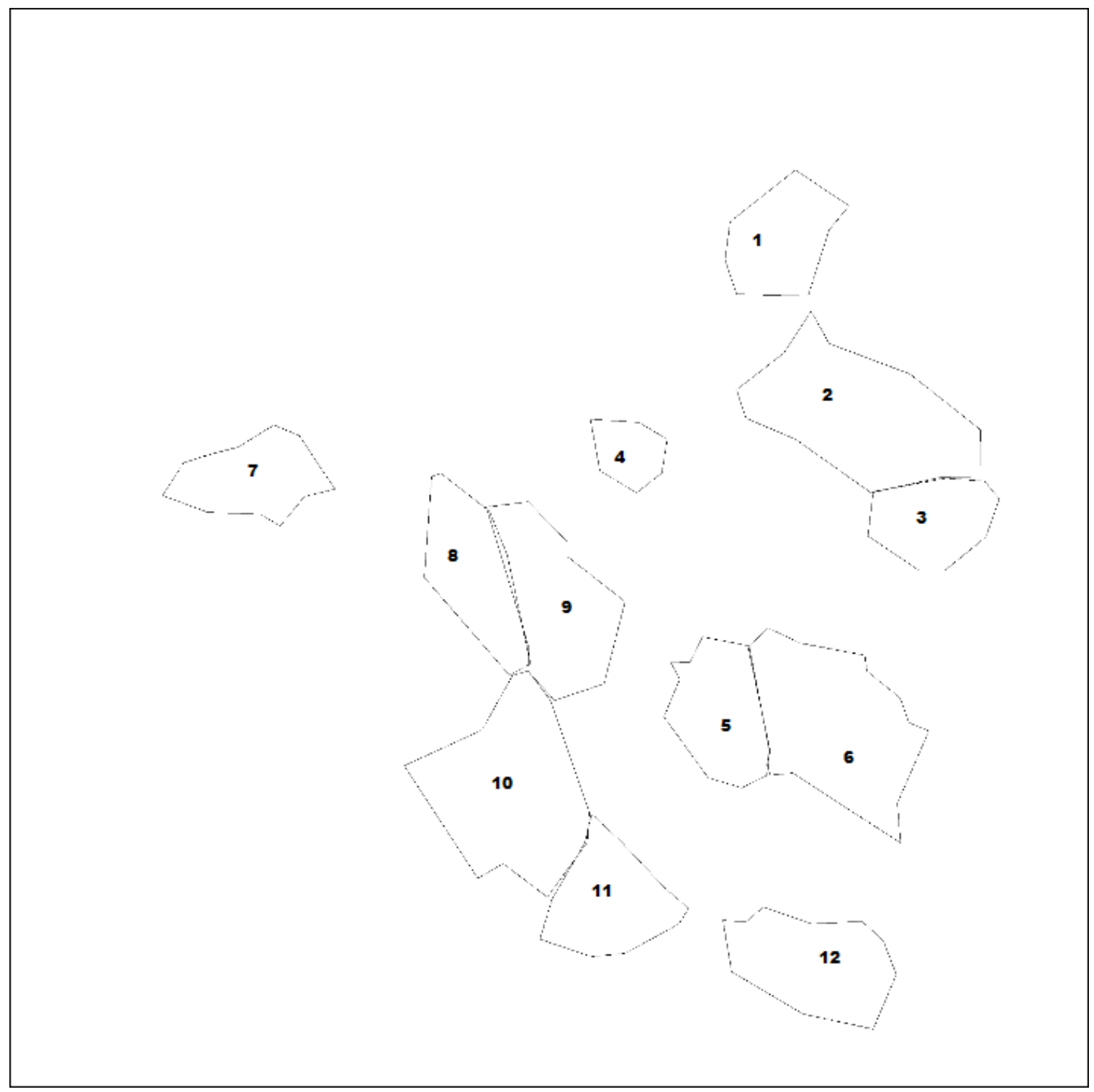

FIGURE A3.10- ARPE 10-2-1 ZO-1 Analysis 


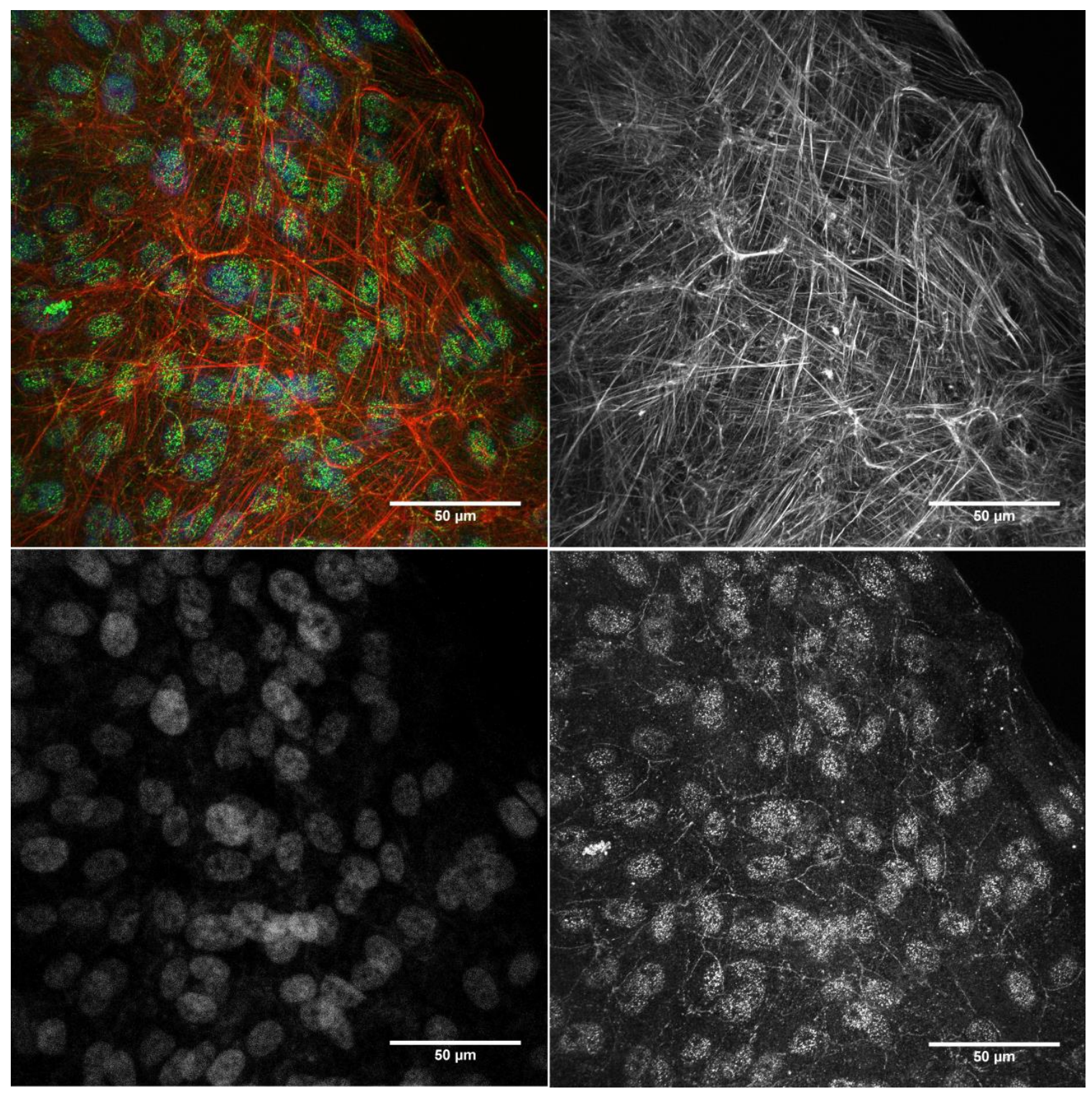

FIGURE A3.11- ARPE 20-1-3 (5/10/13) 


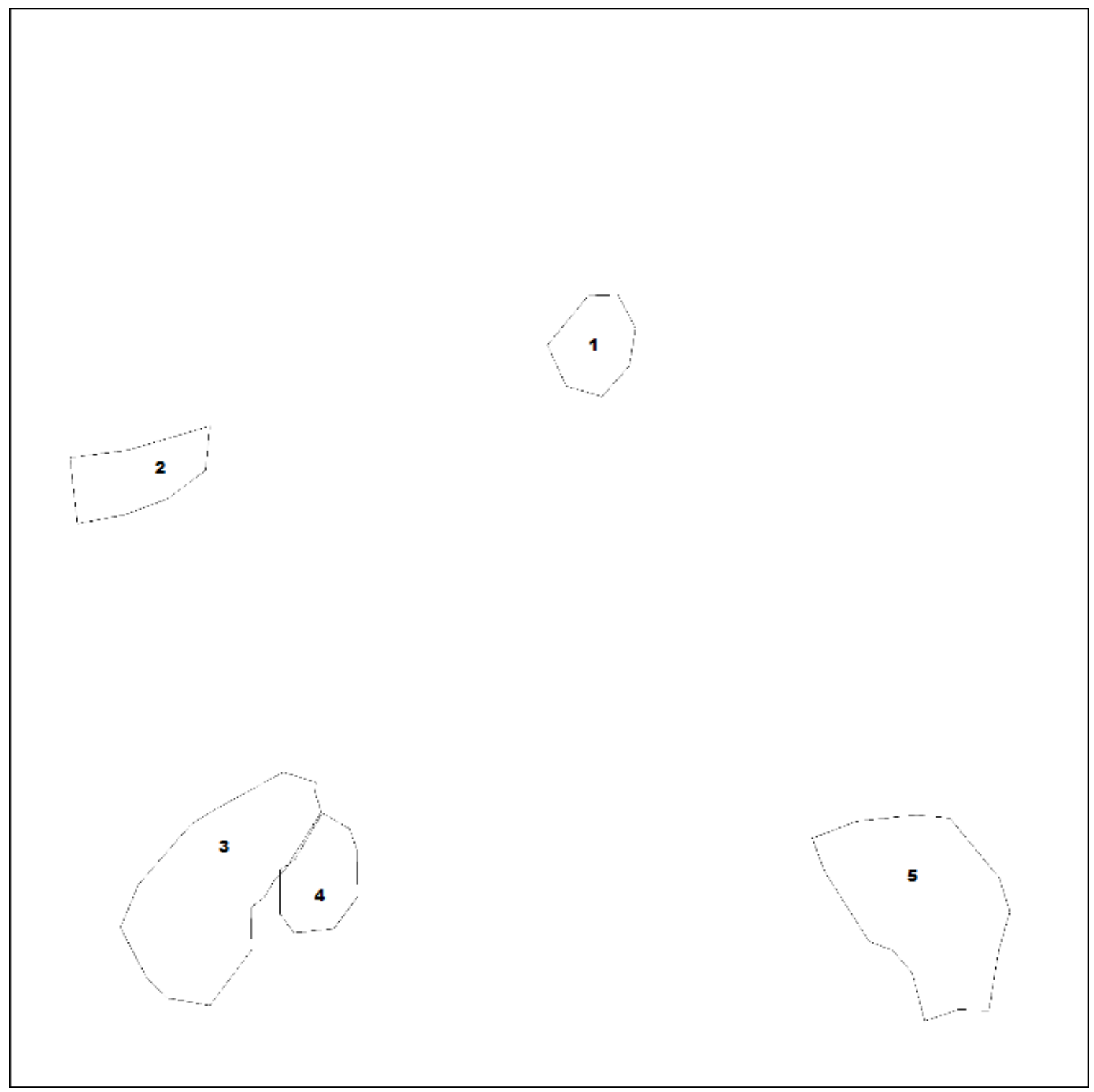

FIGURE A3.12- ARPE 20-1-3 ZO-1 Analysis 


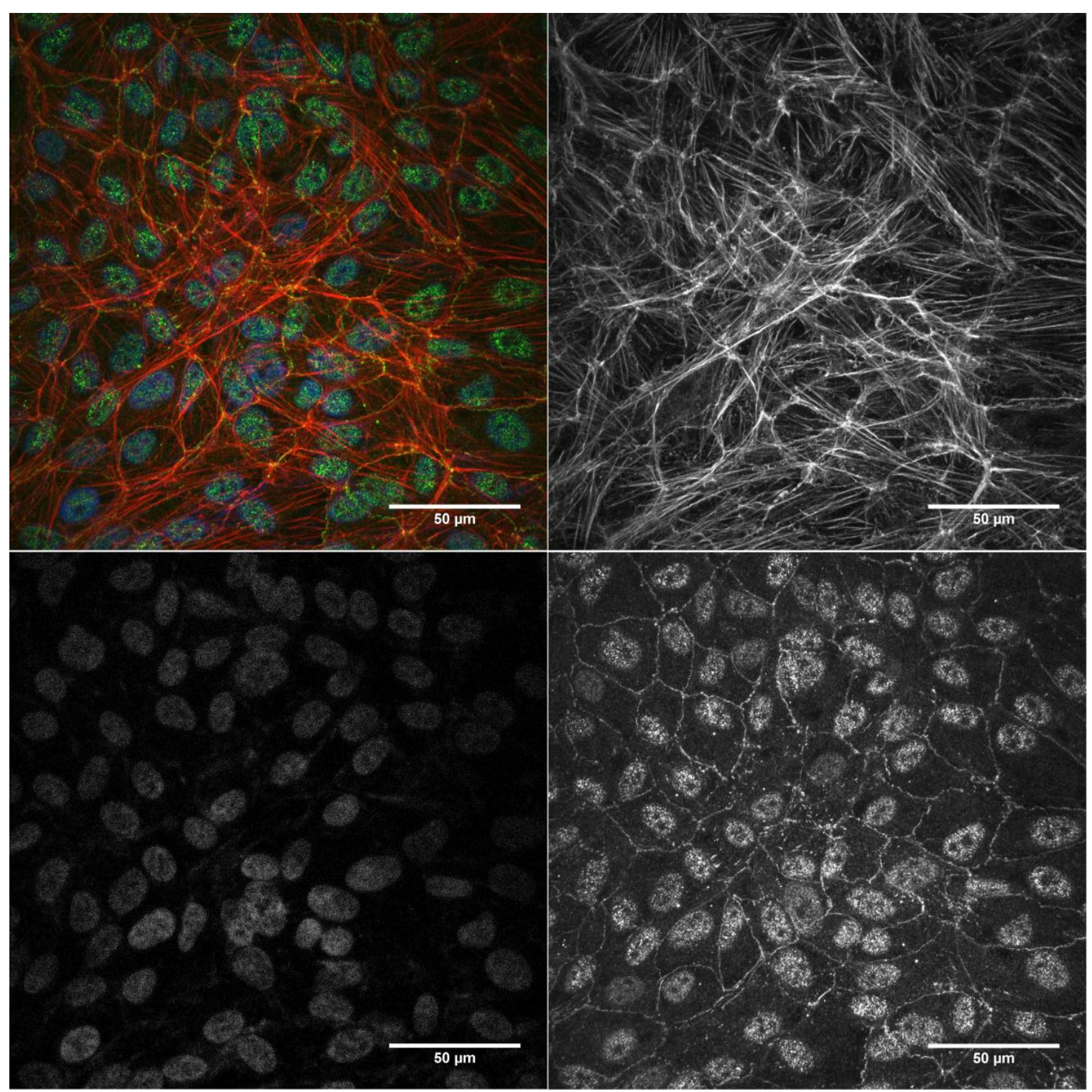

FIGURE A3.13- ARPE 20-2-1 (5/10/13) 


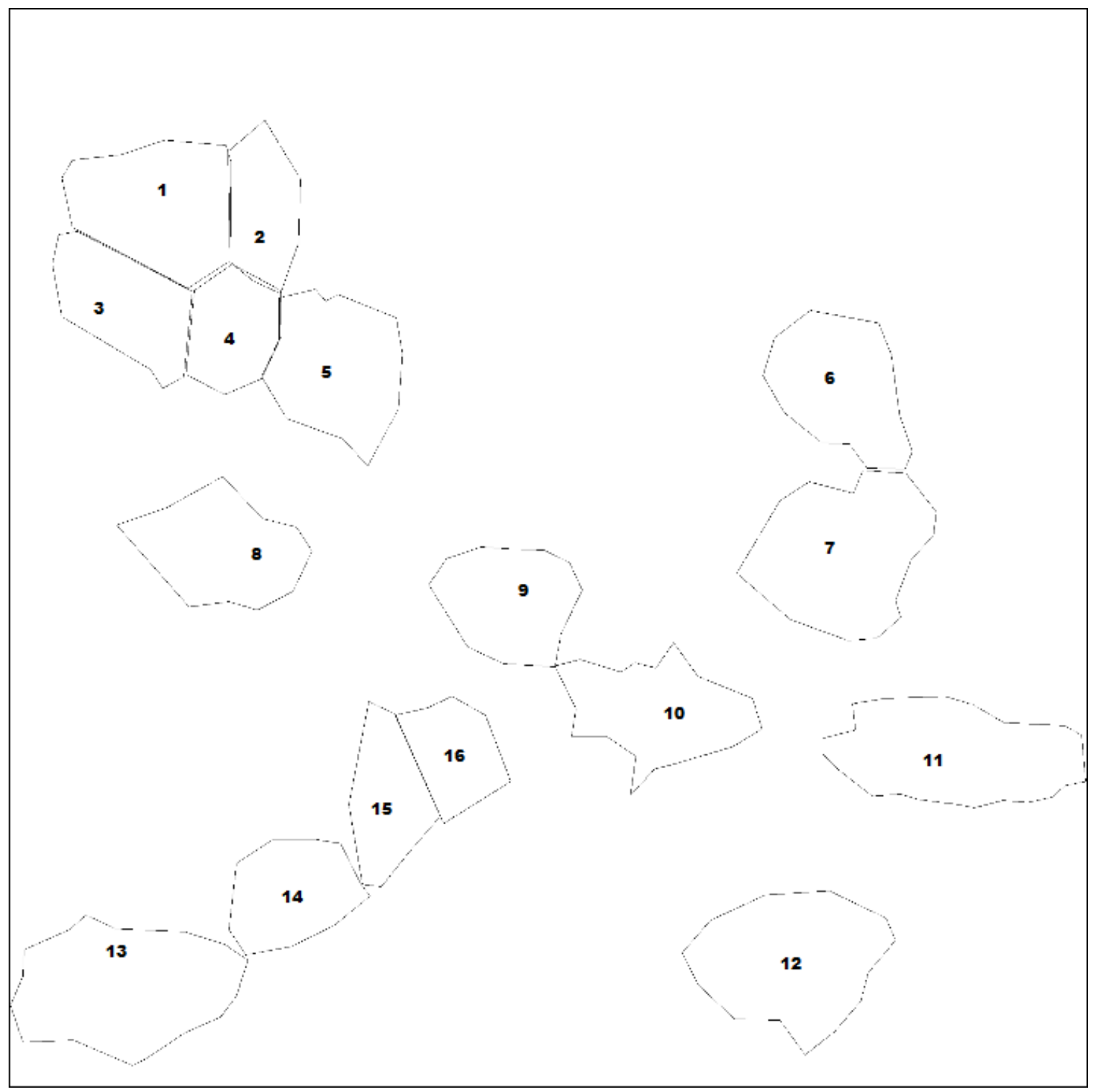

FIGURE A3.14-ARPE 20-2-1 ZO-1 Analysis 


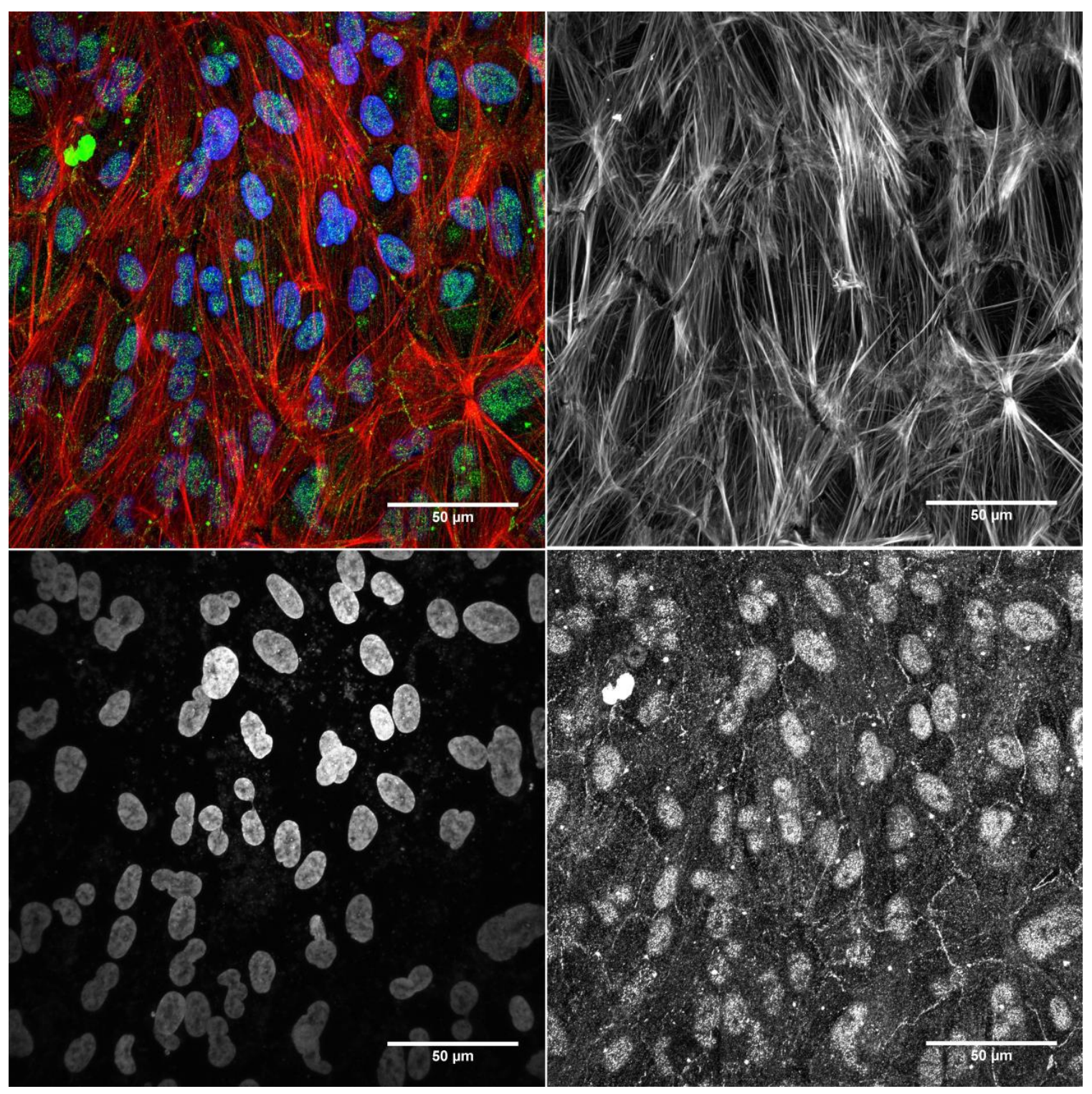

FIGURE A3.15- ARPE FN-1-4 (6/7/13) 


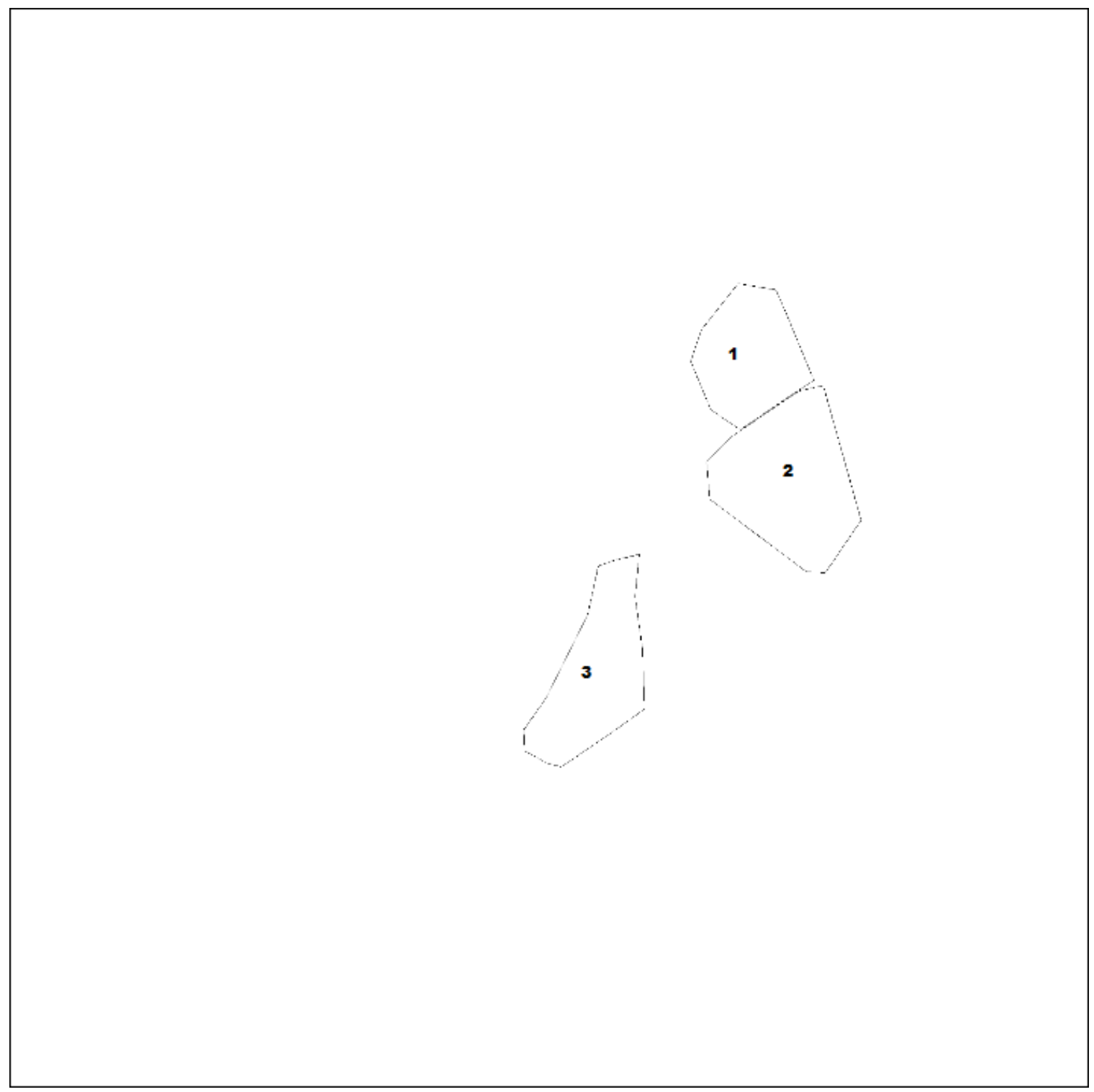

FIGURE A3.16- ARPE FN-1-4 ZO-1 Analysis 


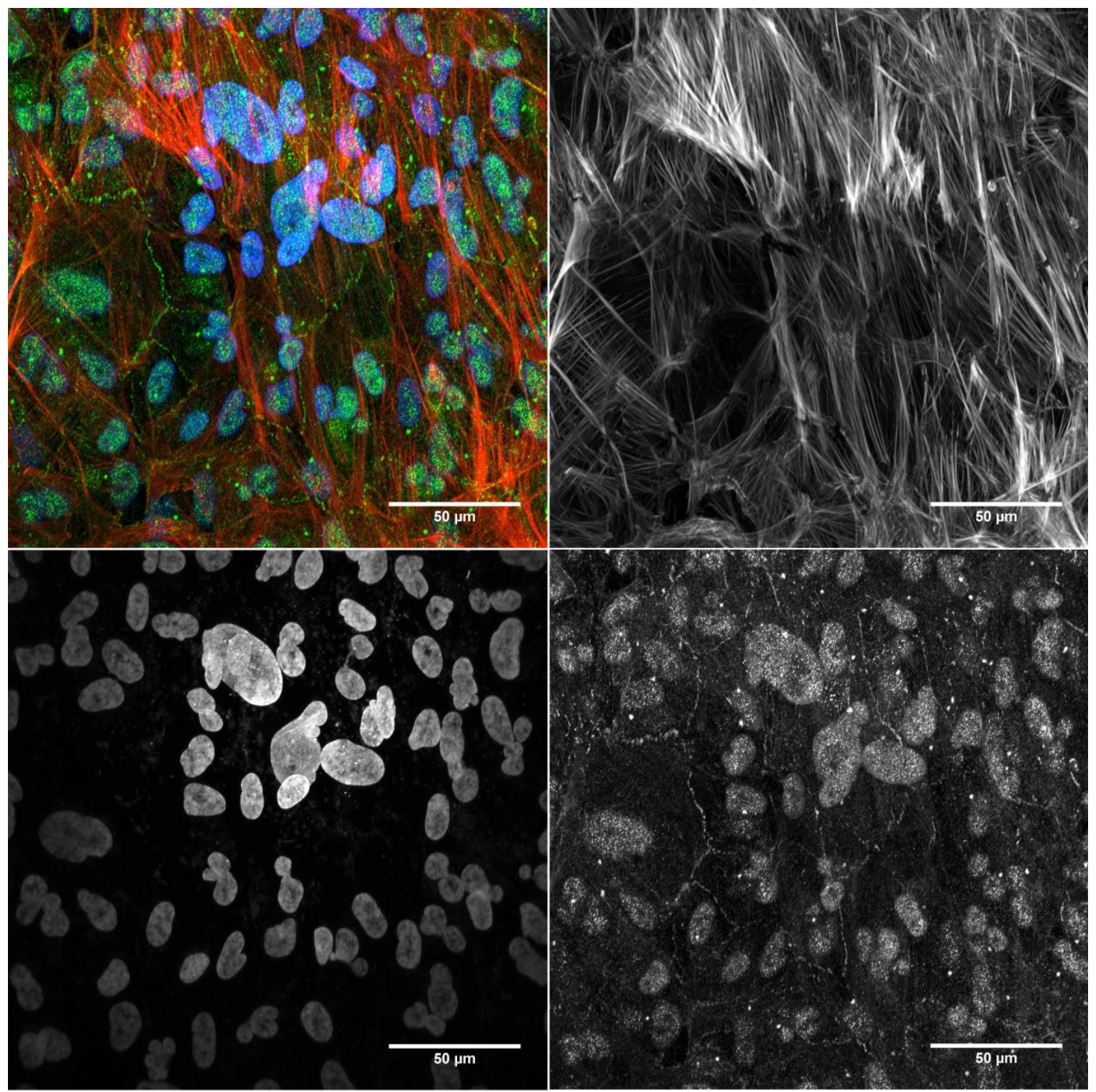

FIGURE A3.17- ARPE FN-3-1 (6/7/13) 


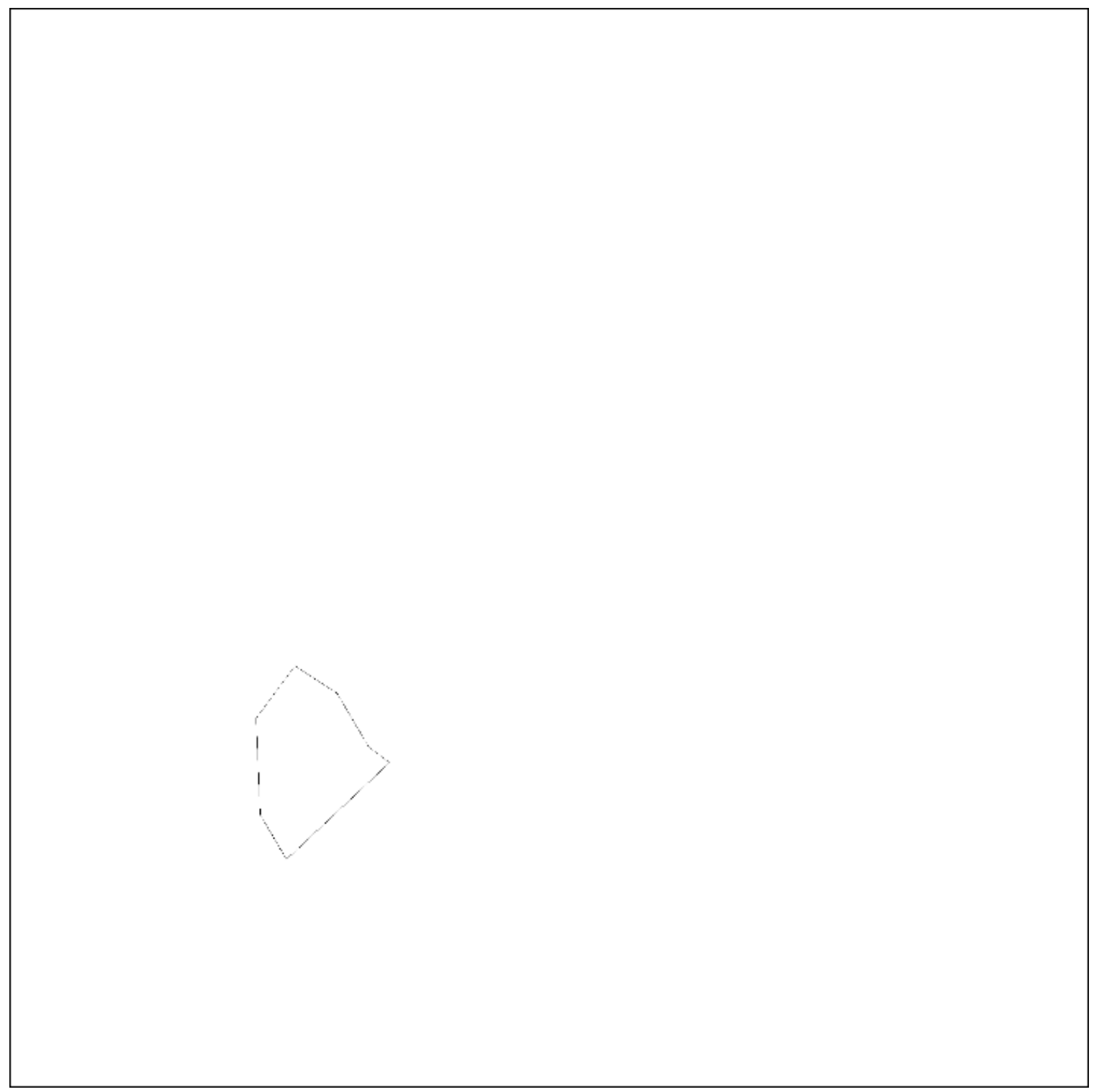

FIGURE A3.18- ARPE FN-3-1 ZO-1 Analysis 


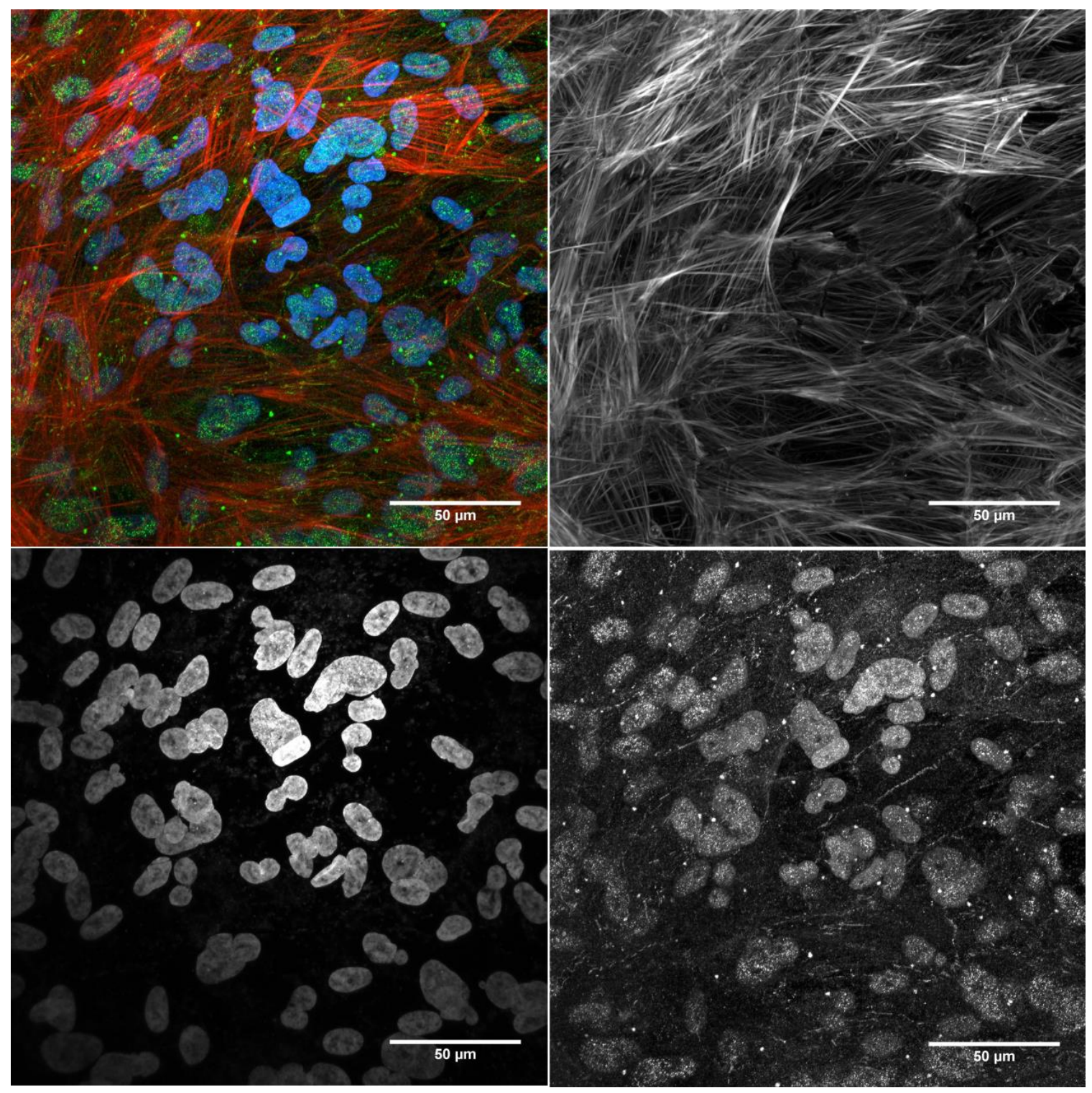

FIGURE A3.19- ARPE FN-4-1 (6/7/13) 


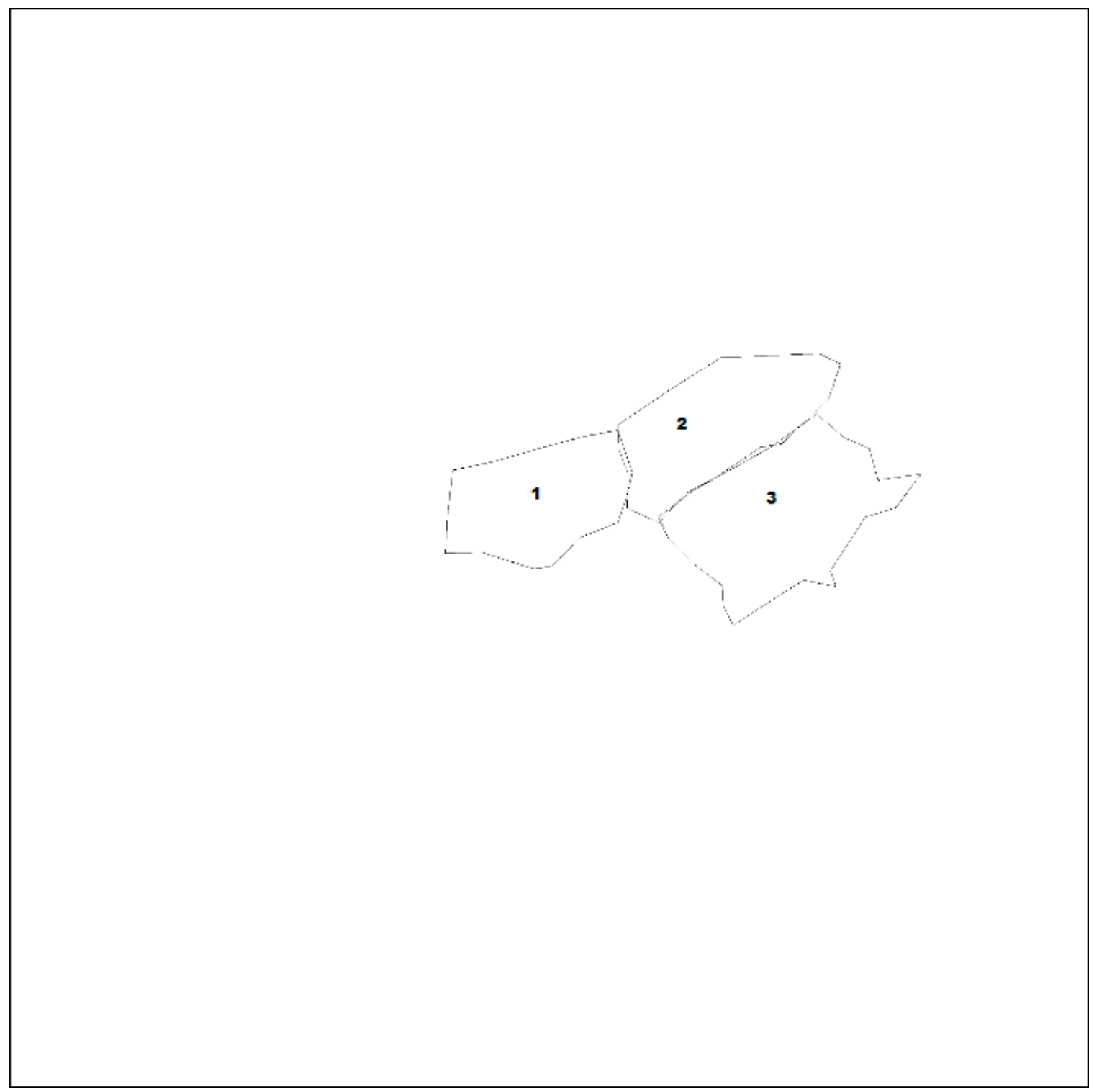

FIGURE A3.20- ARPE FN-4-1 ZO-1 Analysis 


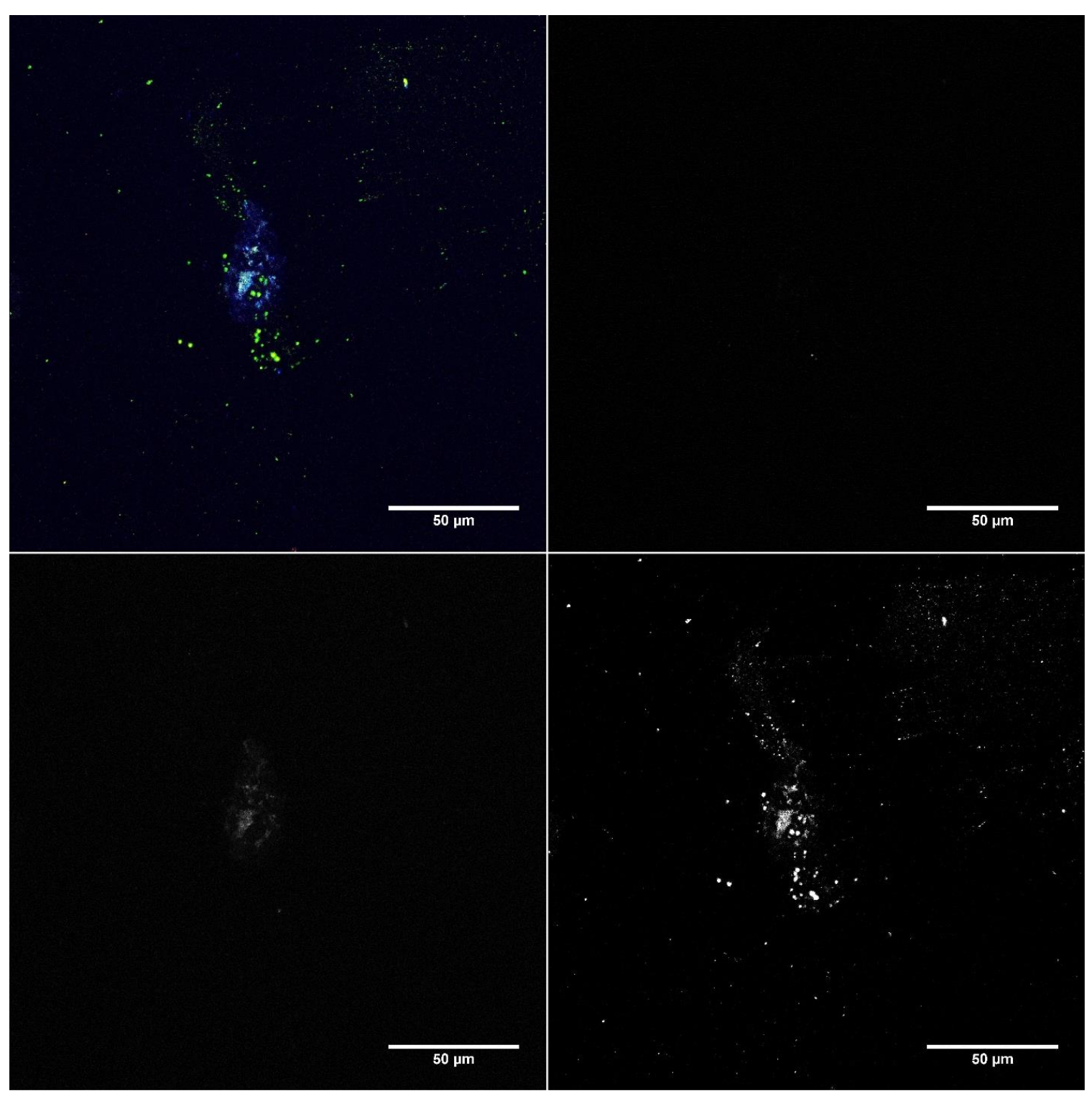

FIGURE A3.21- PRPE 0-1-1 (5/17/13) 


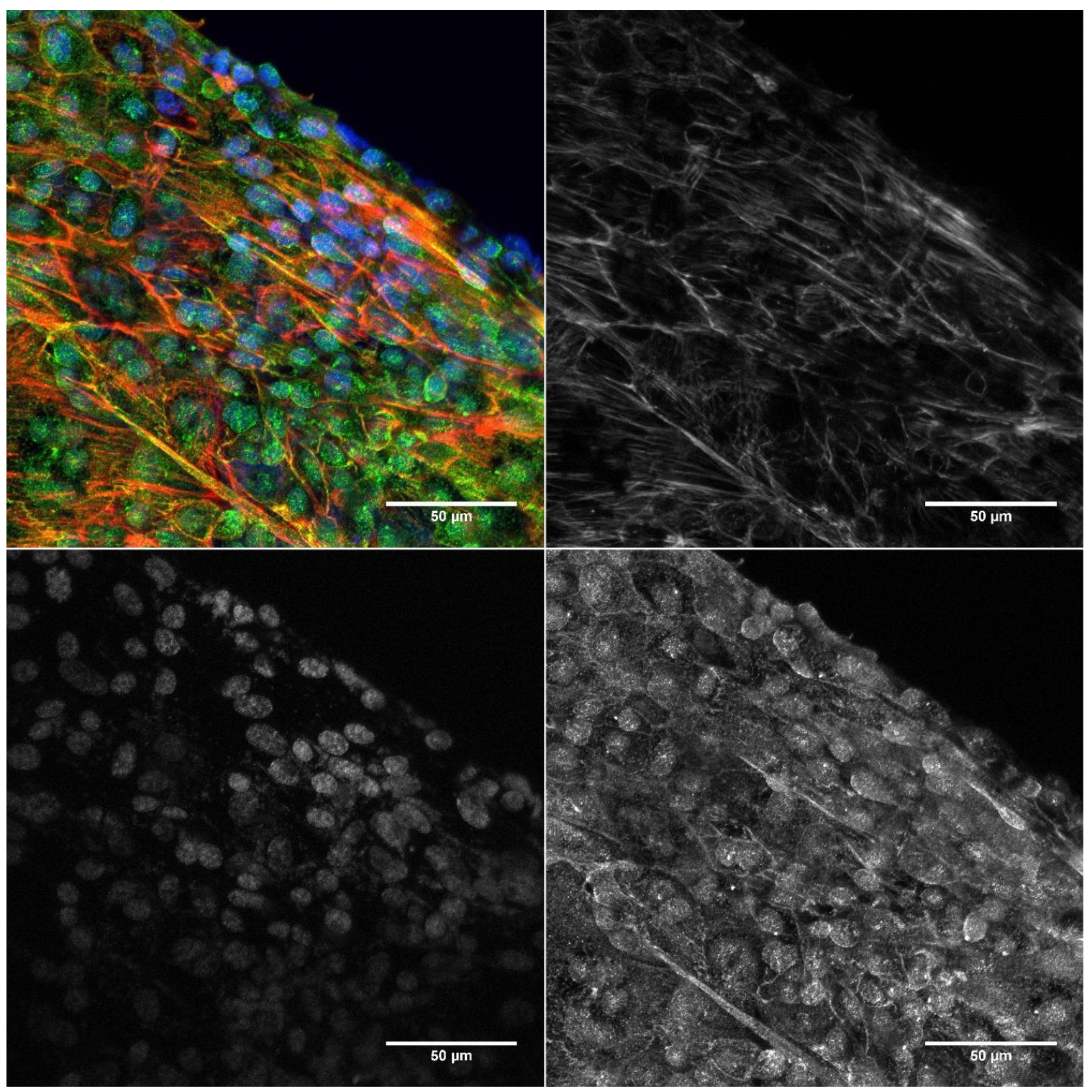

FIGURE A3.22- PRPE 0-2-1 (5/17/13) 


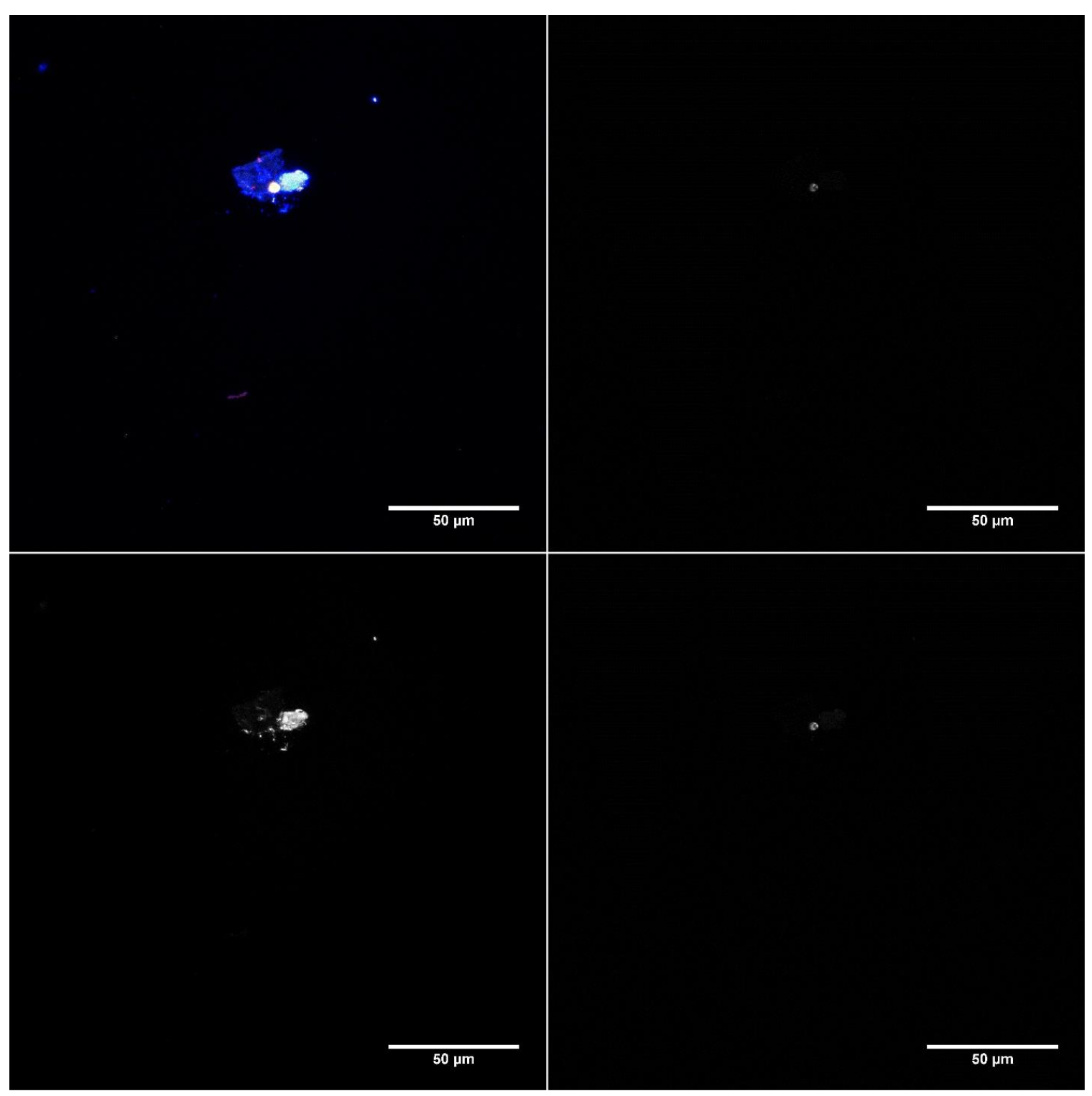

FIGURE A3.23- PRPE 0-3-1 (6/1/13) 


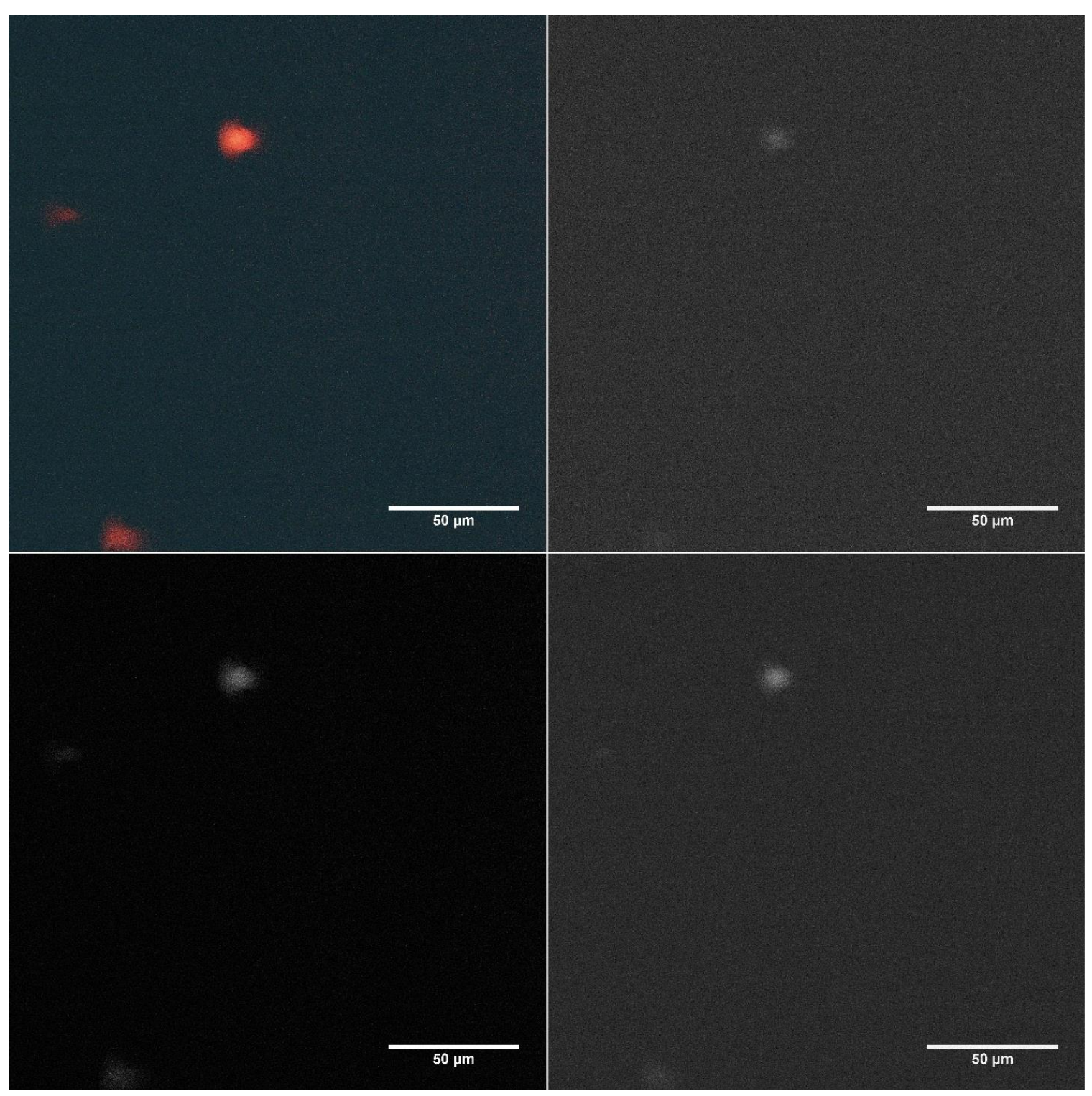

FIGURE A3.24- PRPE 0-4-1 (6/1/13) 


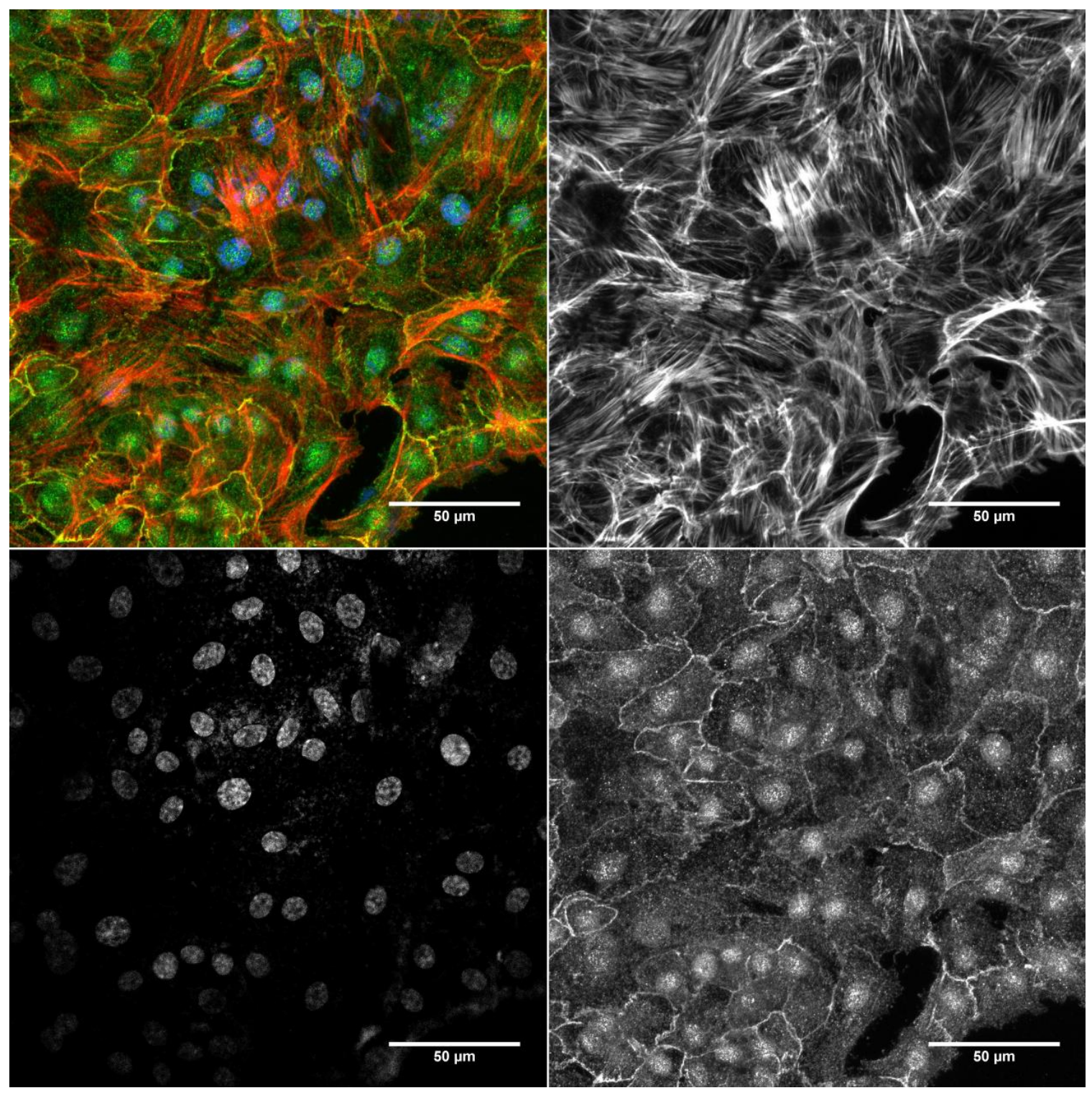

FIGURE A3.25- PRPE 5-1-1 (5/17/13) 


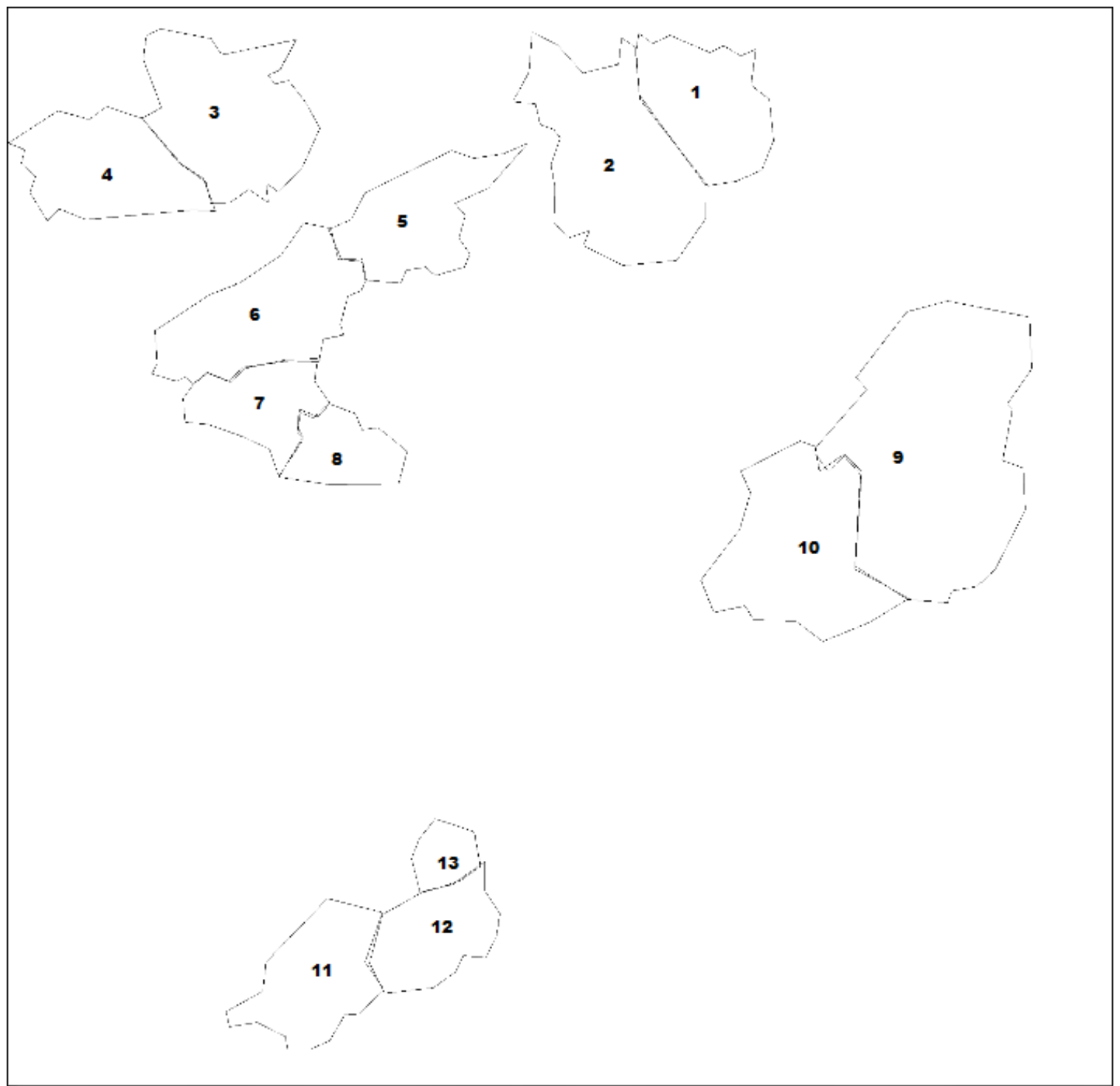

FIGURE A3.26- PRPE 5-1-1 ZO-1 Analysis 


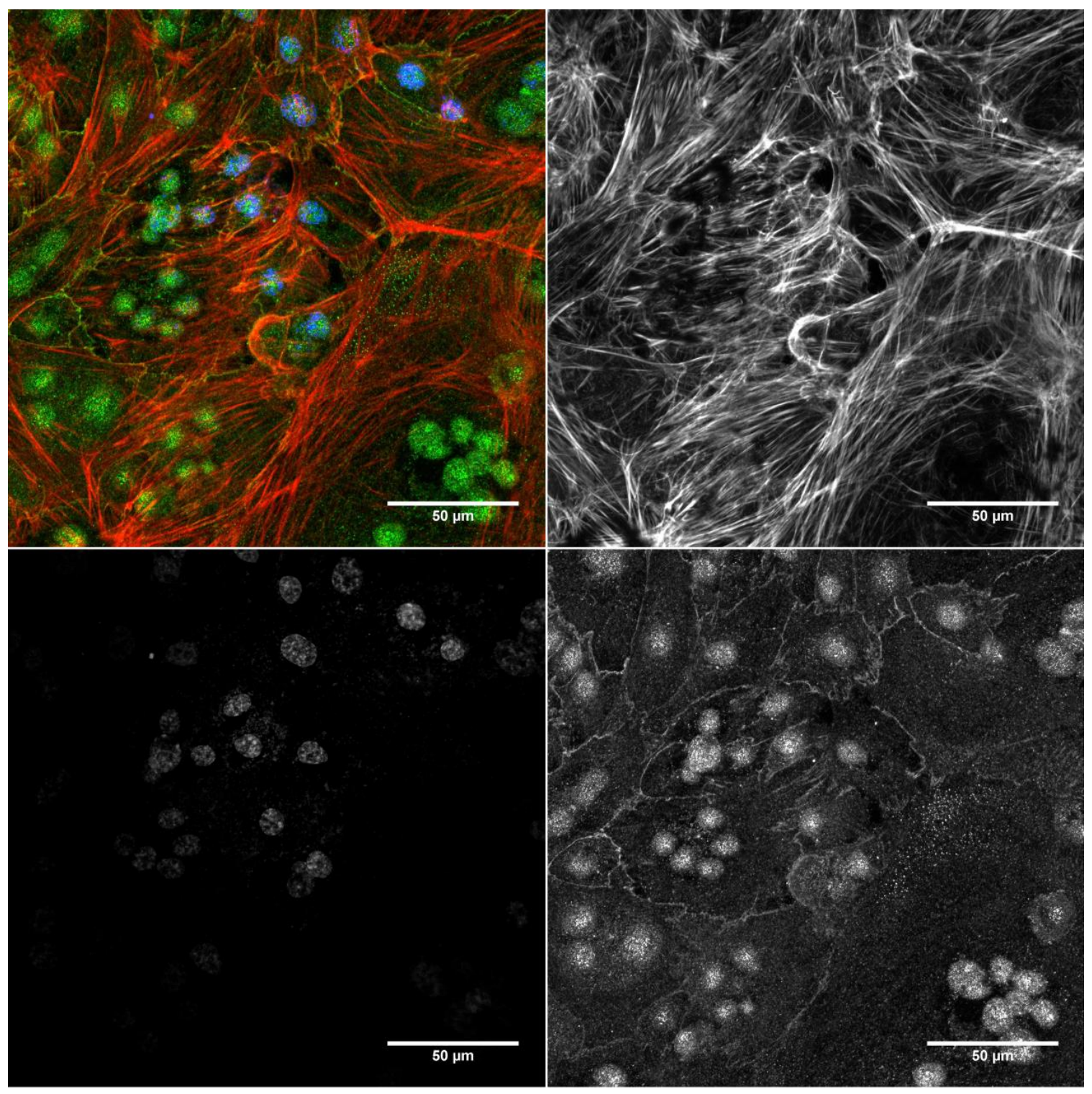

FIGURE A3.27- PRPE 5-2-1 (5/17/13) 


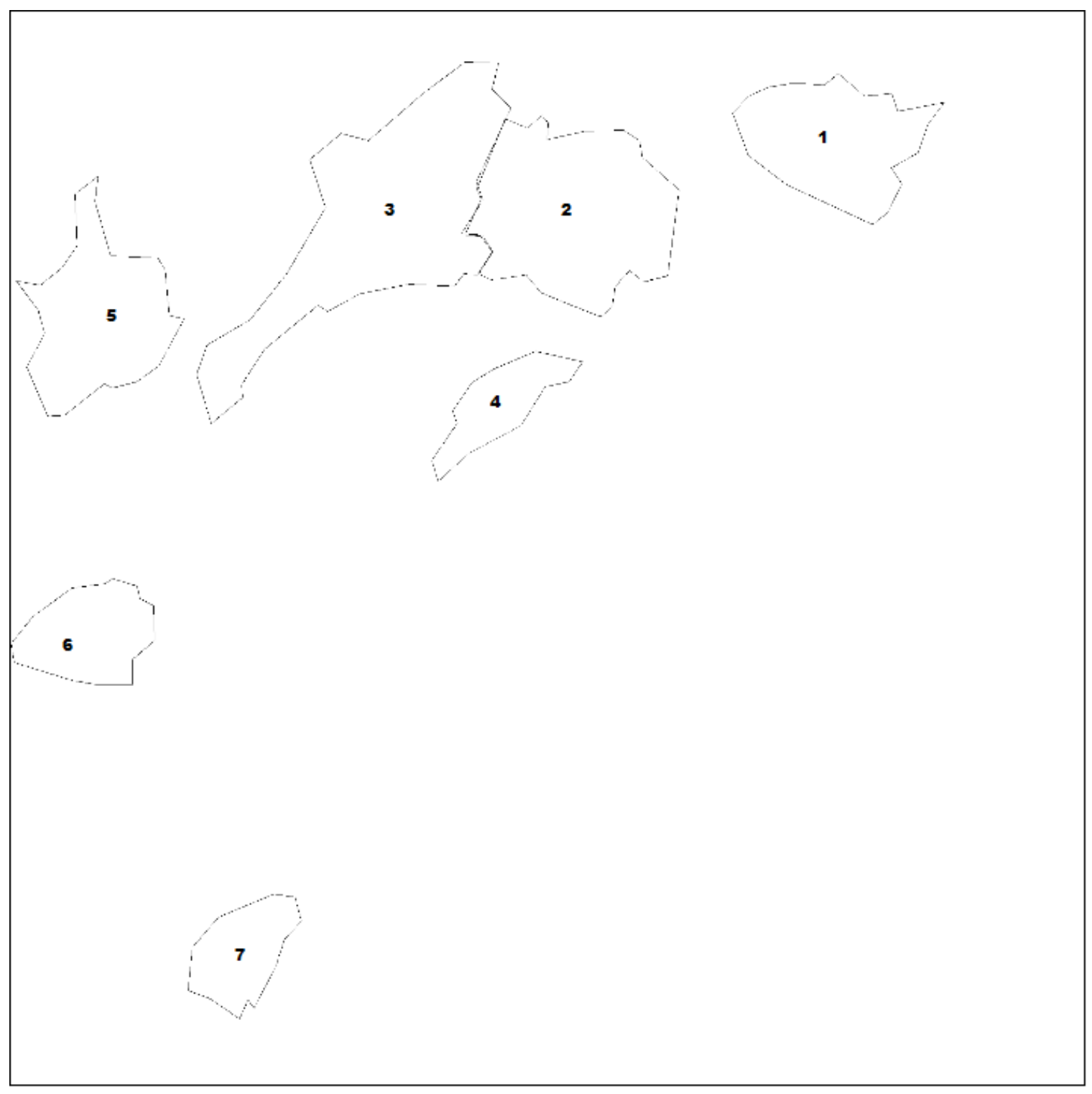

FIGURE A3.28- PRPE 5-2-1 ZO-1 Analysis 


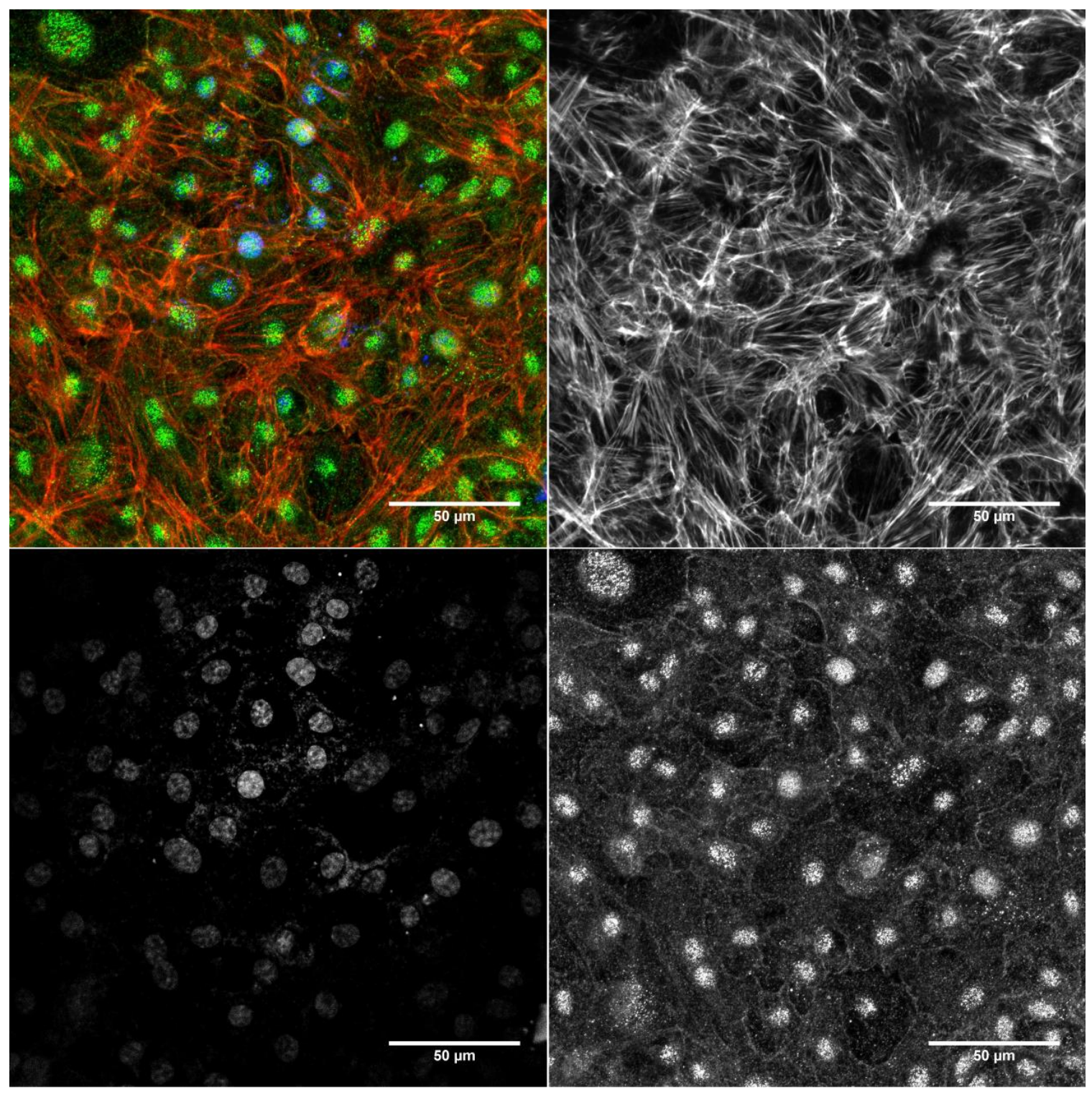

FIGURE A3.29- PRPE 5-3-1 (6/1/13) 


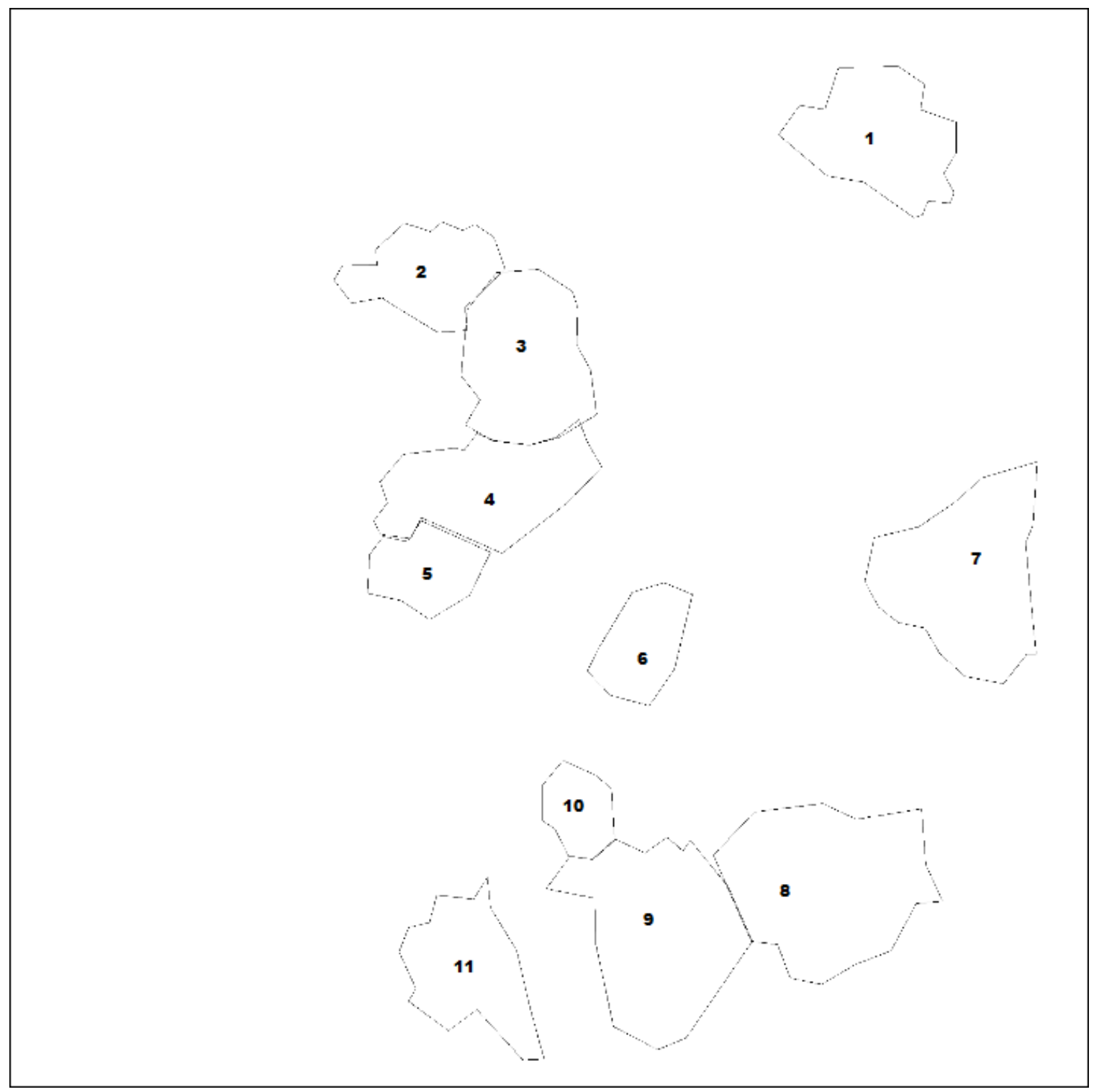

FIGURE A3.30- PRPE 5-3-1 ZO-1 Analysis 


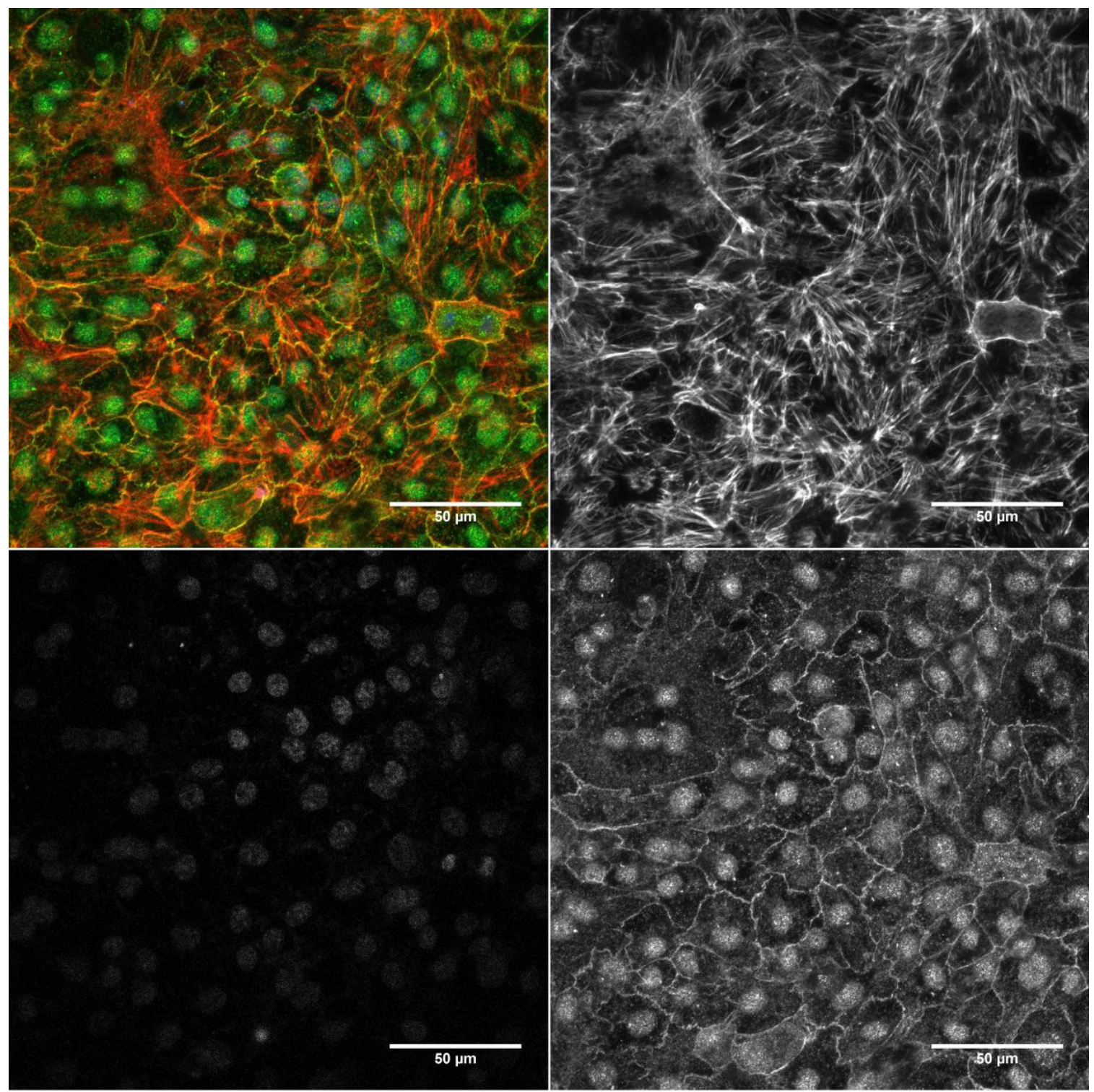

FIGURE A3.31- PRPE 10-1-1 (5/17/13) 


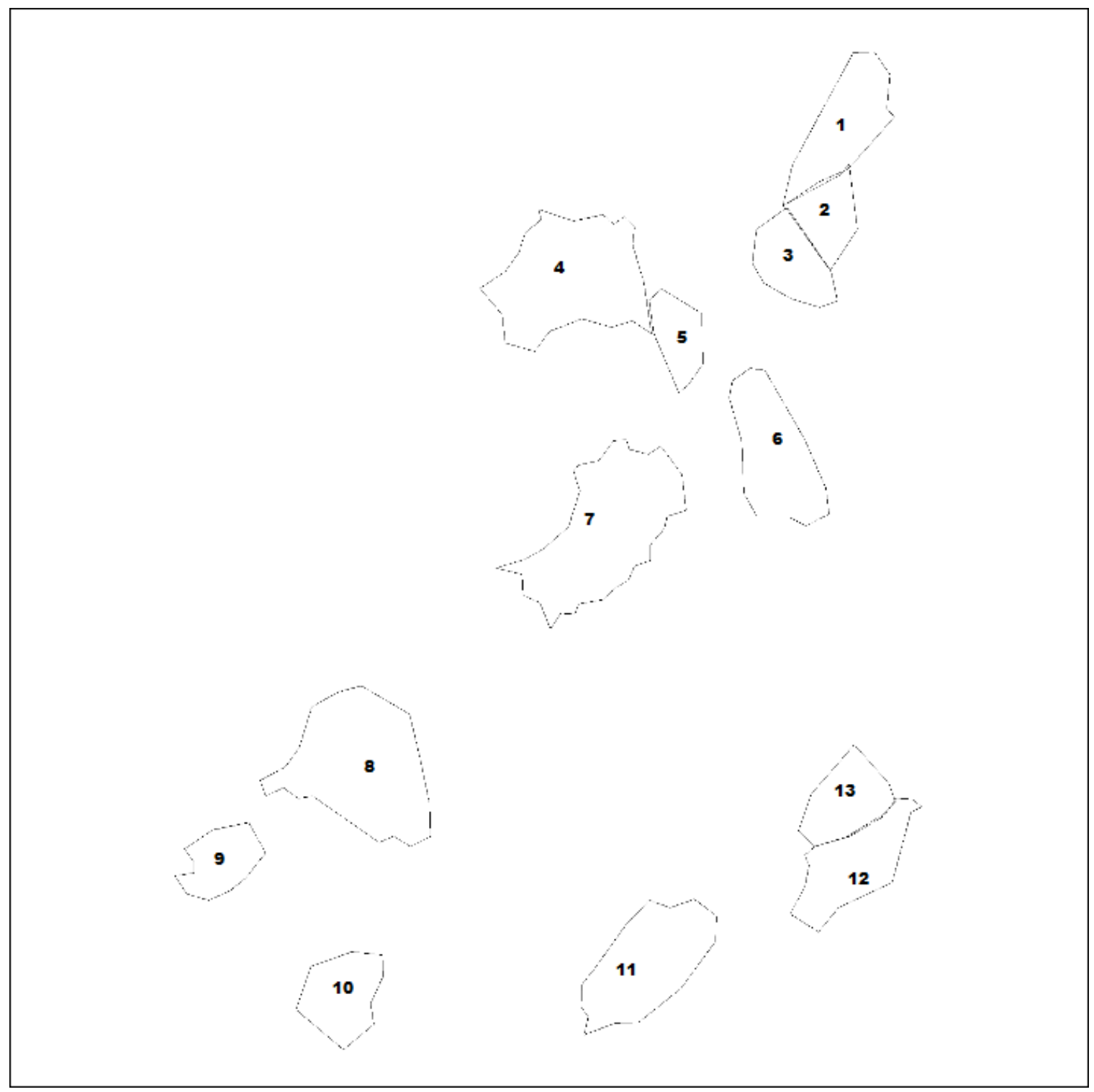

FIGURE A3.32- PRPE 10-1-1 ZO-1 Analysis 


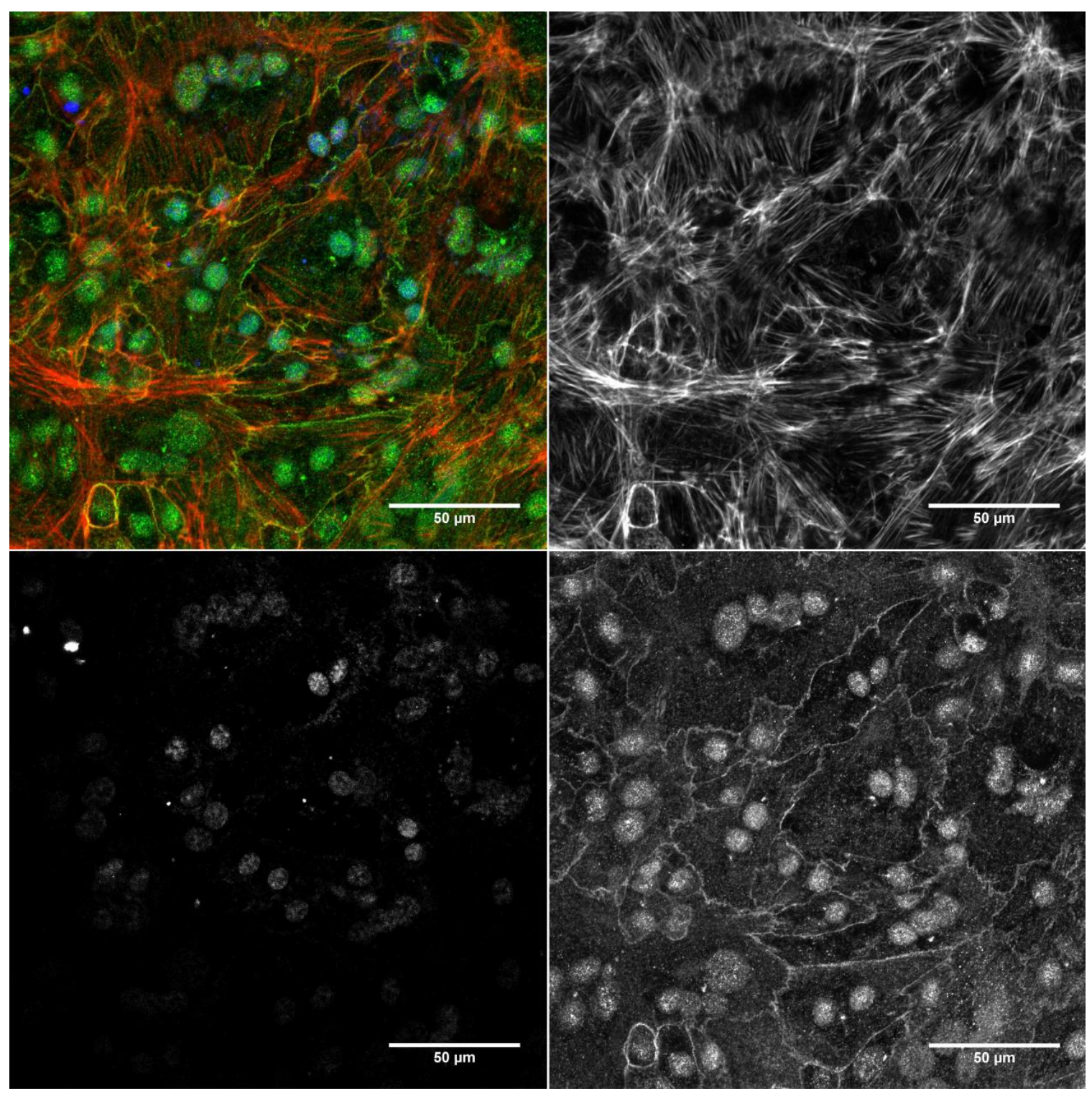

FIGURE A3.33- PRPE 10-2-1 (5/17/13) 


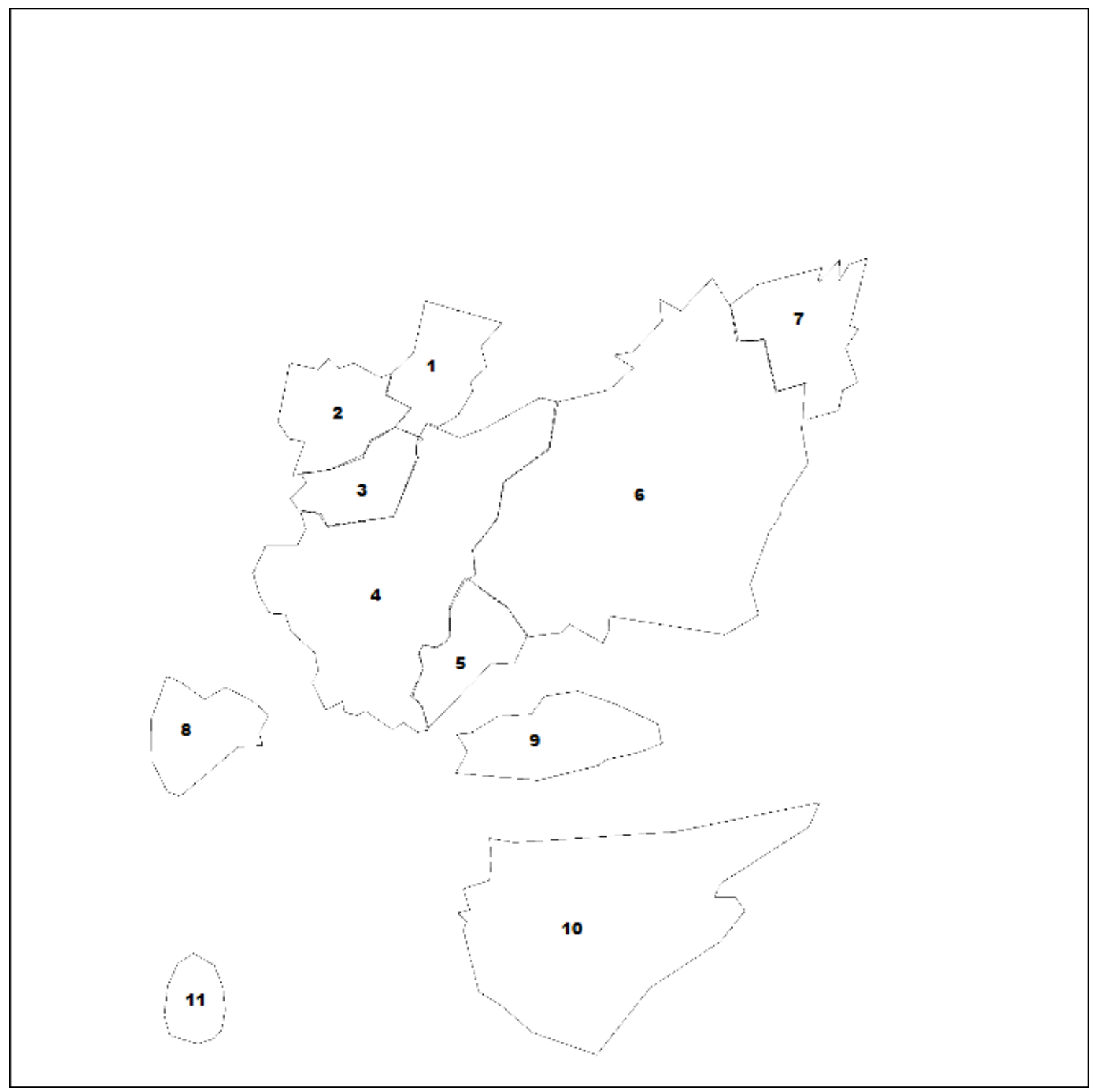

FIGURE A3.34- PRPE 10-2-1 ZO-1 Analysis 


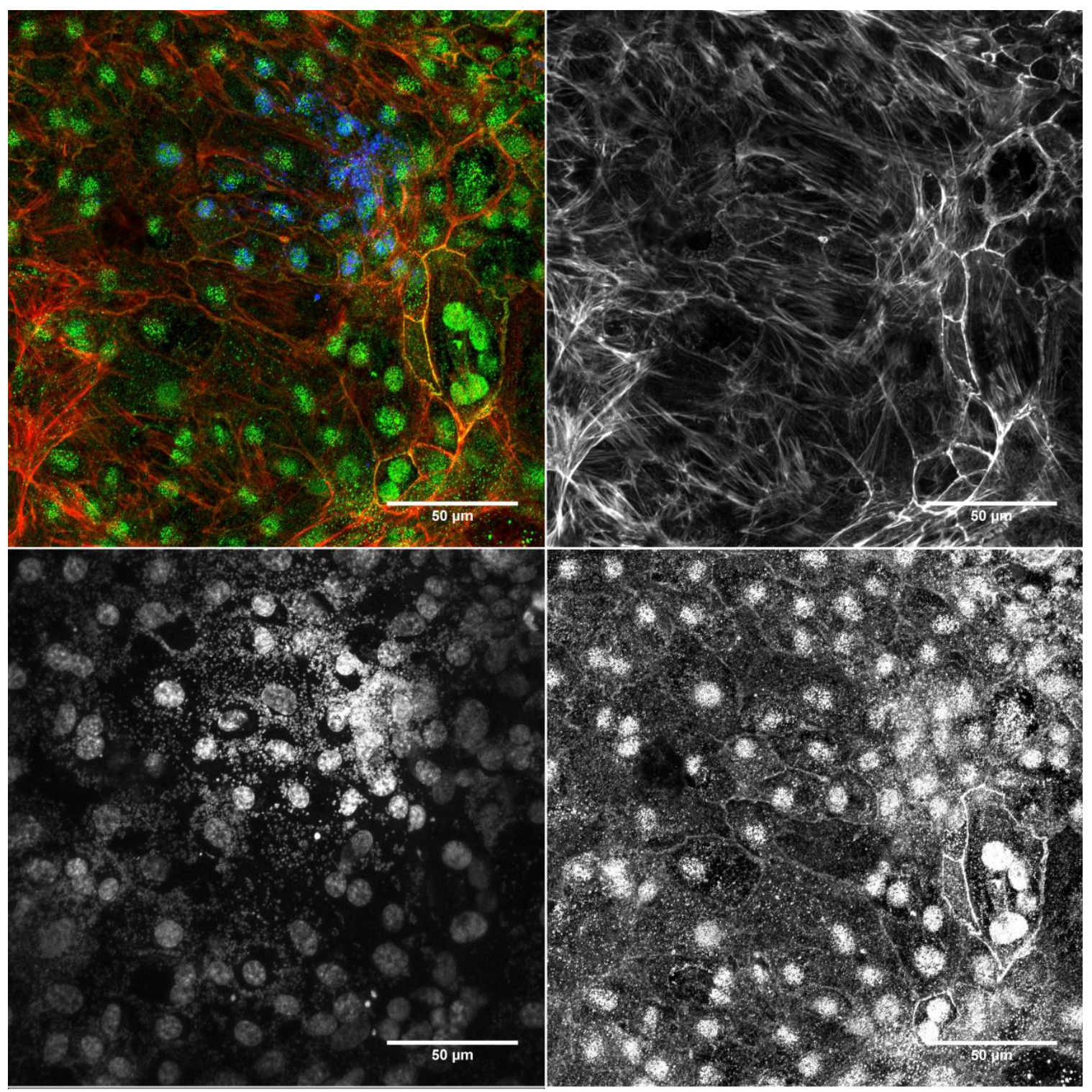

FIGURE A3.35- PRPE 10-3-1 (6/1/13) 


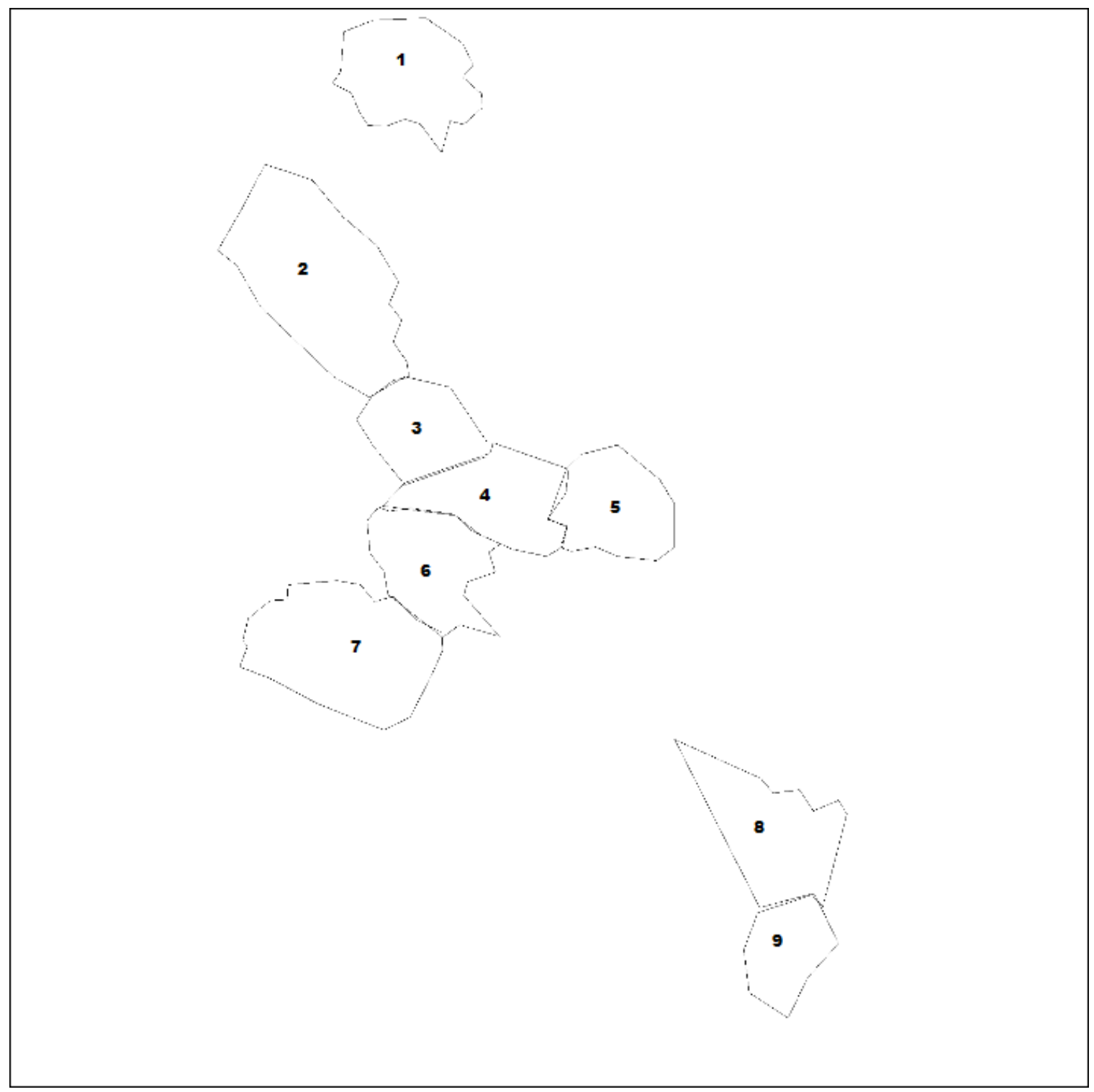

FIGURE A3.36- PRPE 10-3-1 ZO-1 Analysis 


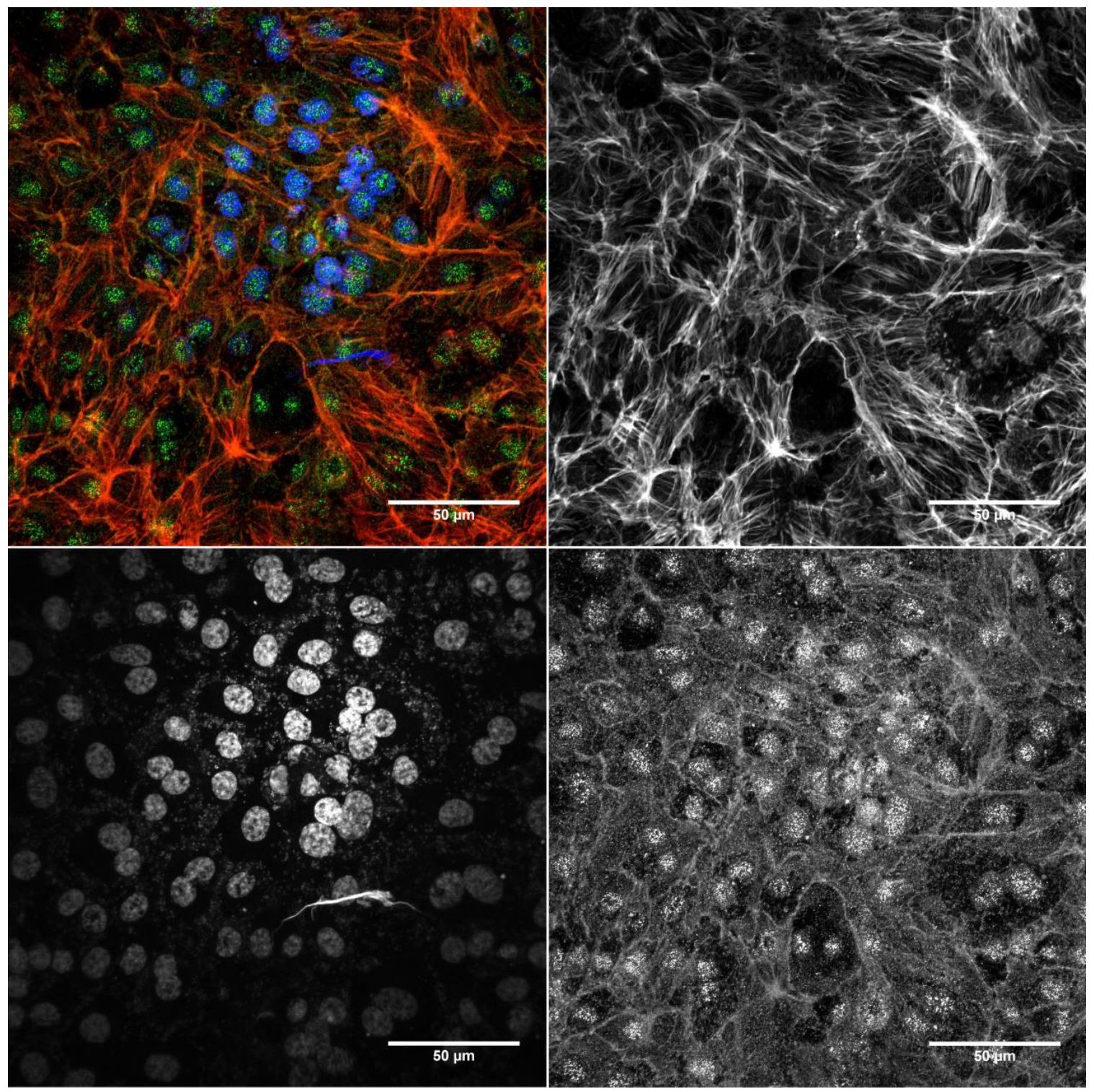

FIGURE A3.37- PRPE 10-4-1 (6/1/13) 


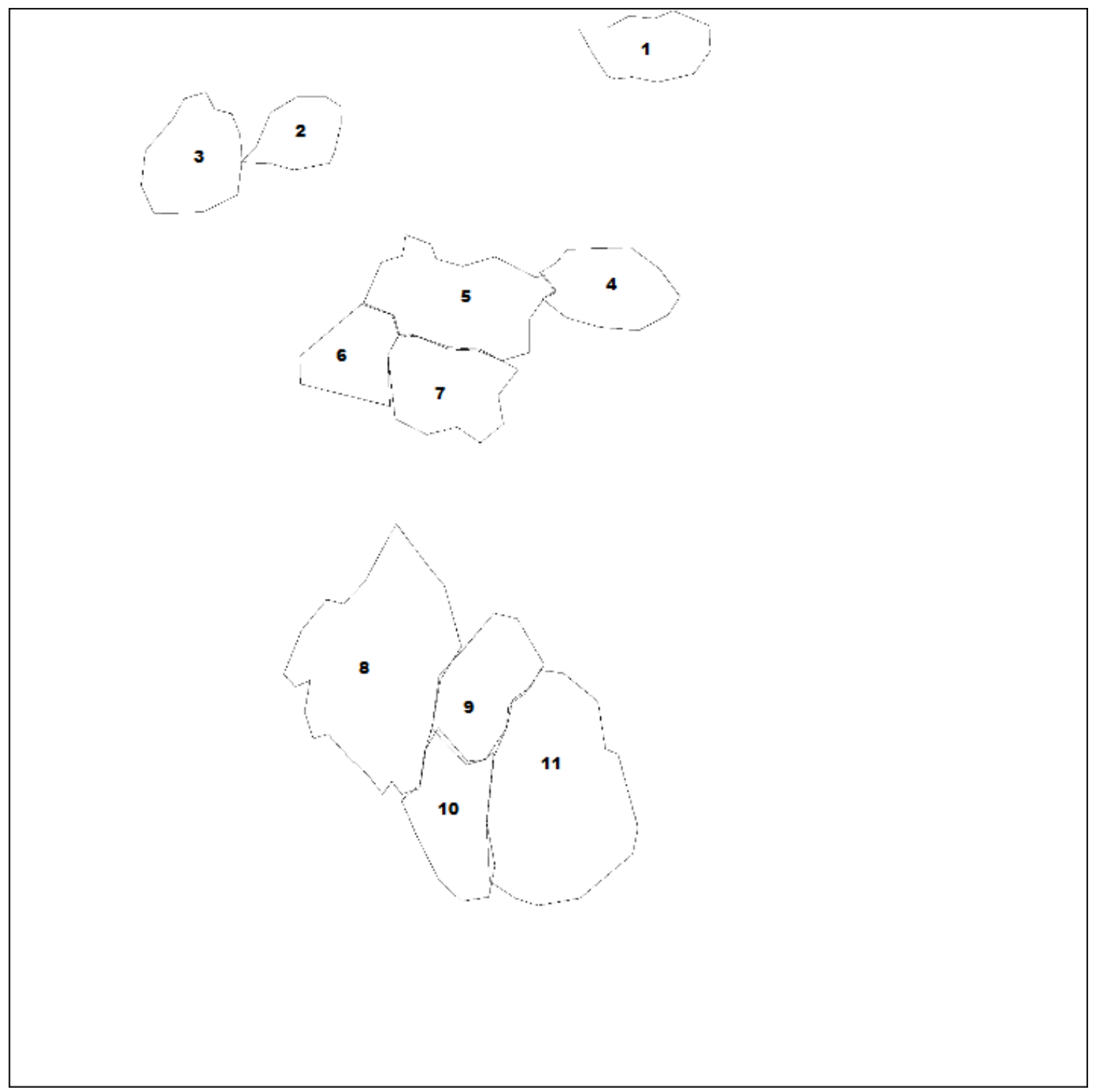

FIGURE A3.38- PRPE 10-4-1 ZO-1 Analysis 


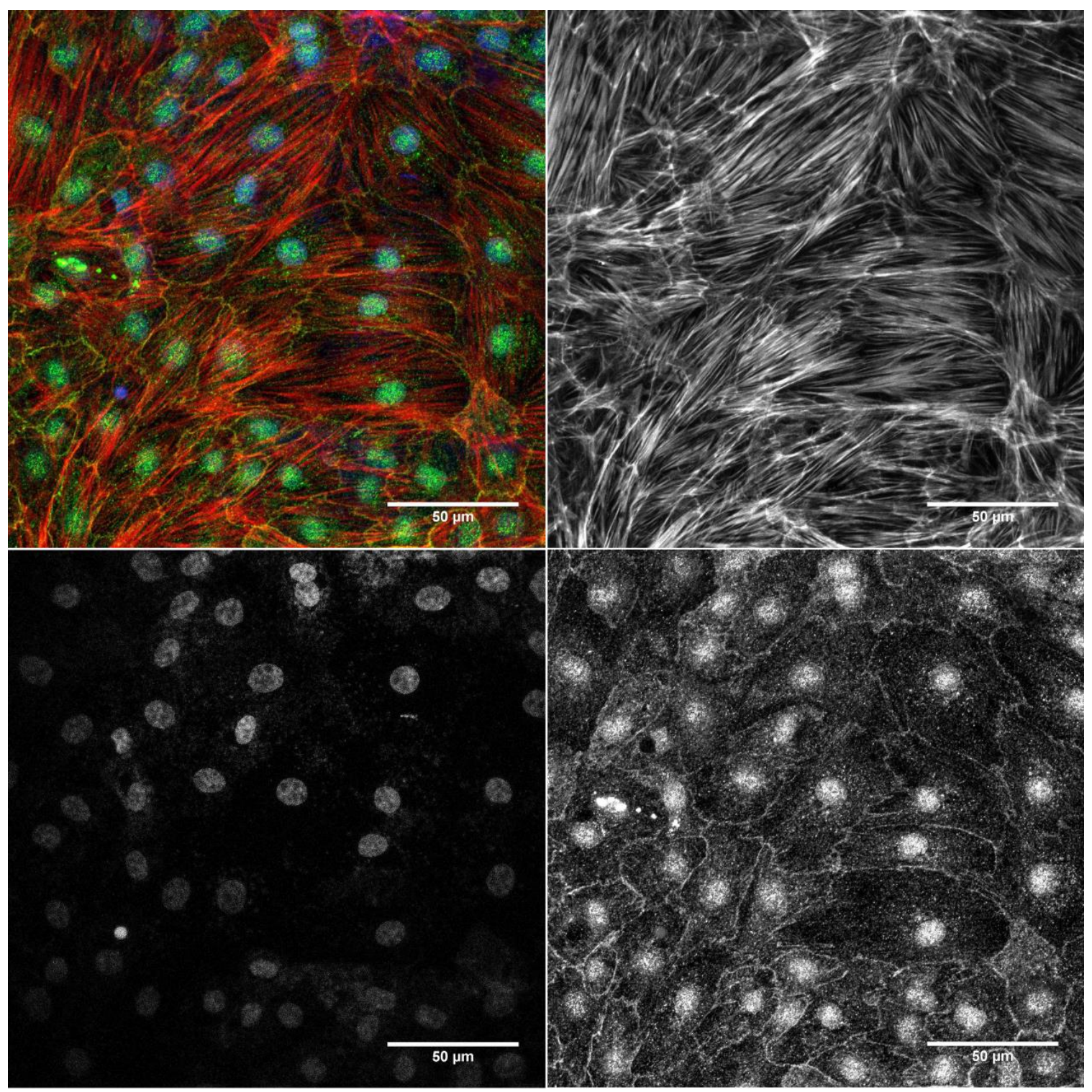

FIGURE A3.39- PRPE 20-1-1 (5/17/13) 


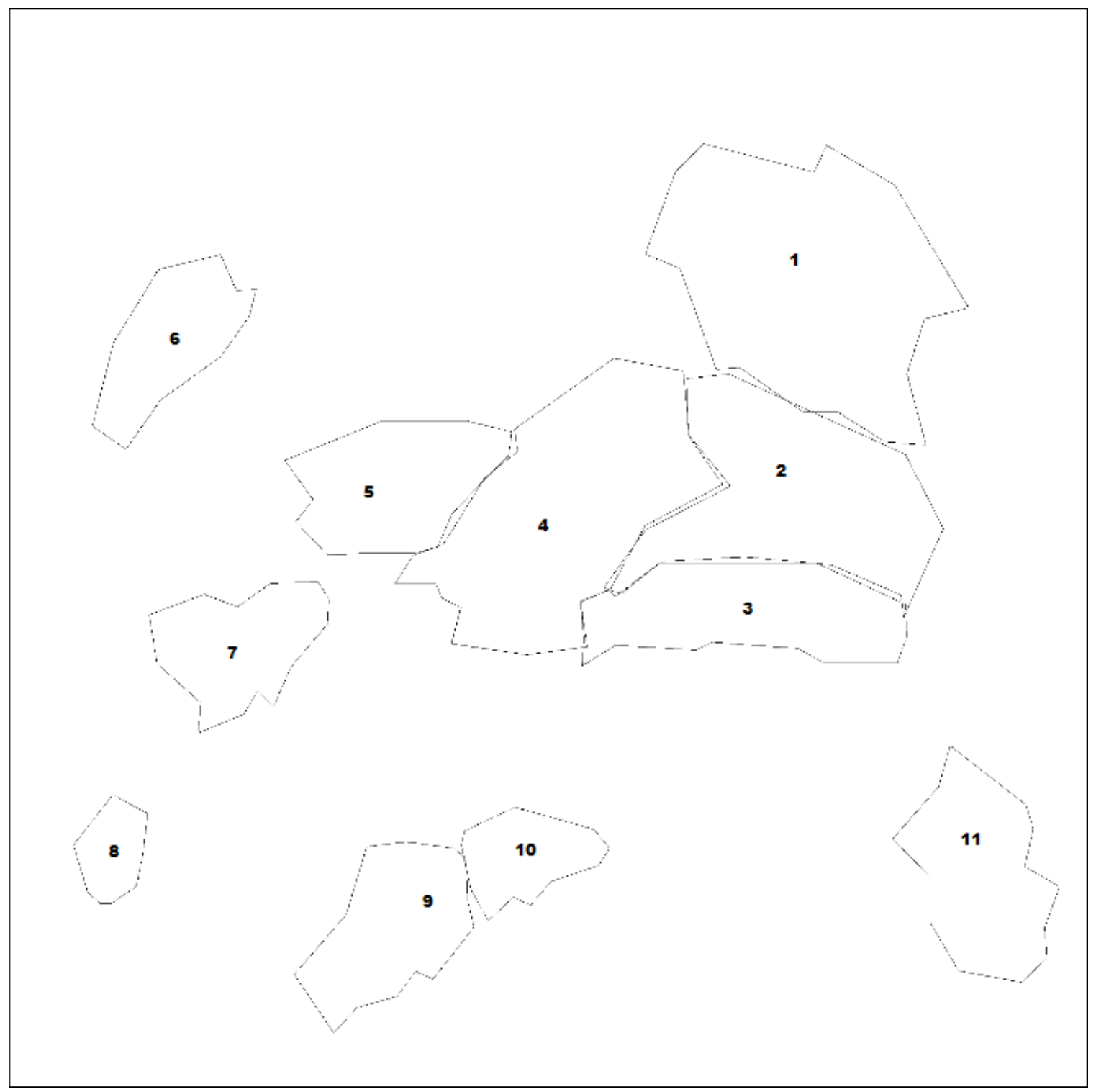

FIGURE A3.40- PRPE 20-1-1 ZO-1 Analysis 


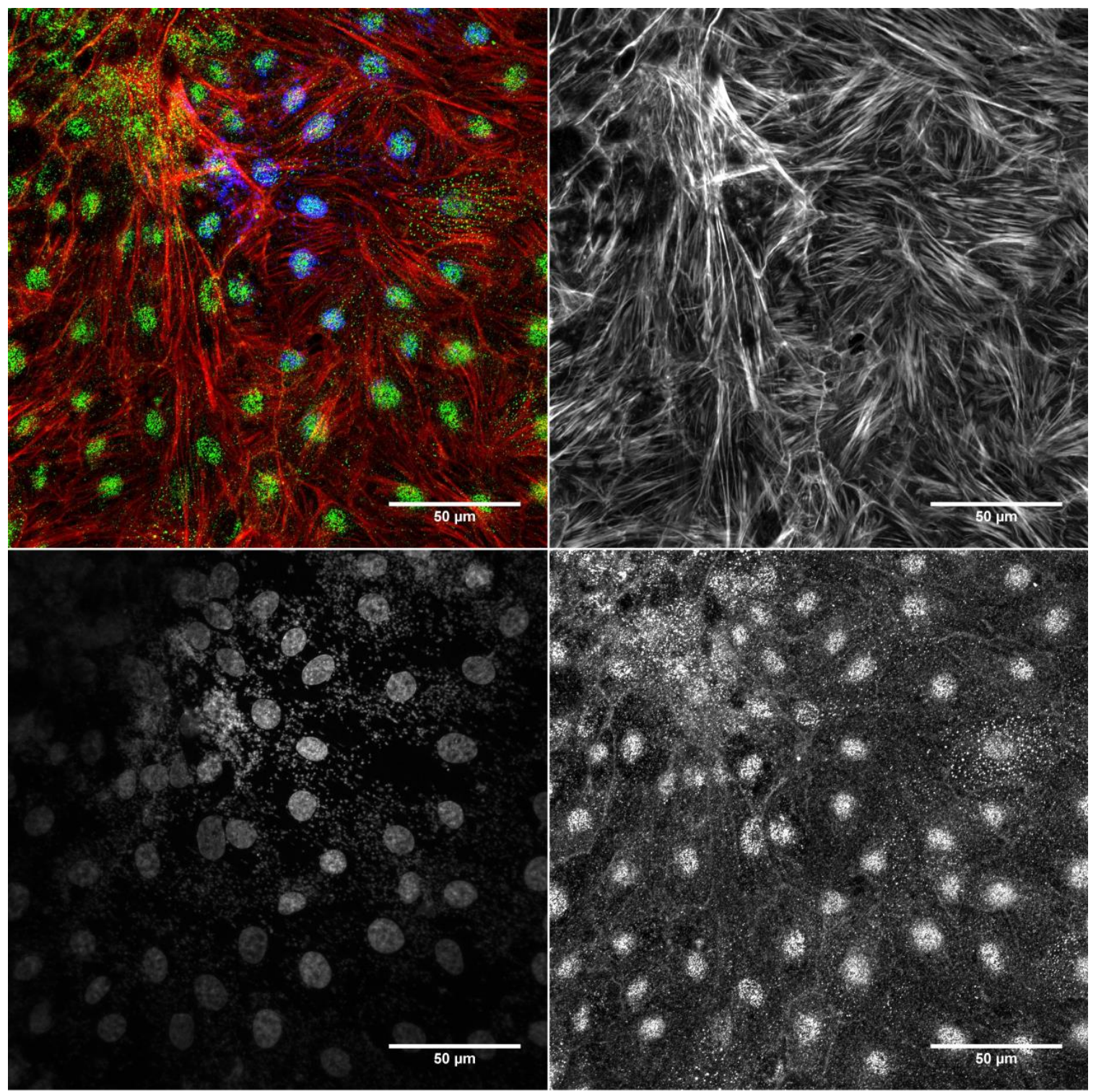

FIGURE A3.41- PRPE 20-1-3 (6/1/13) 


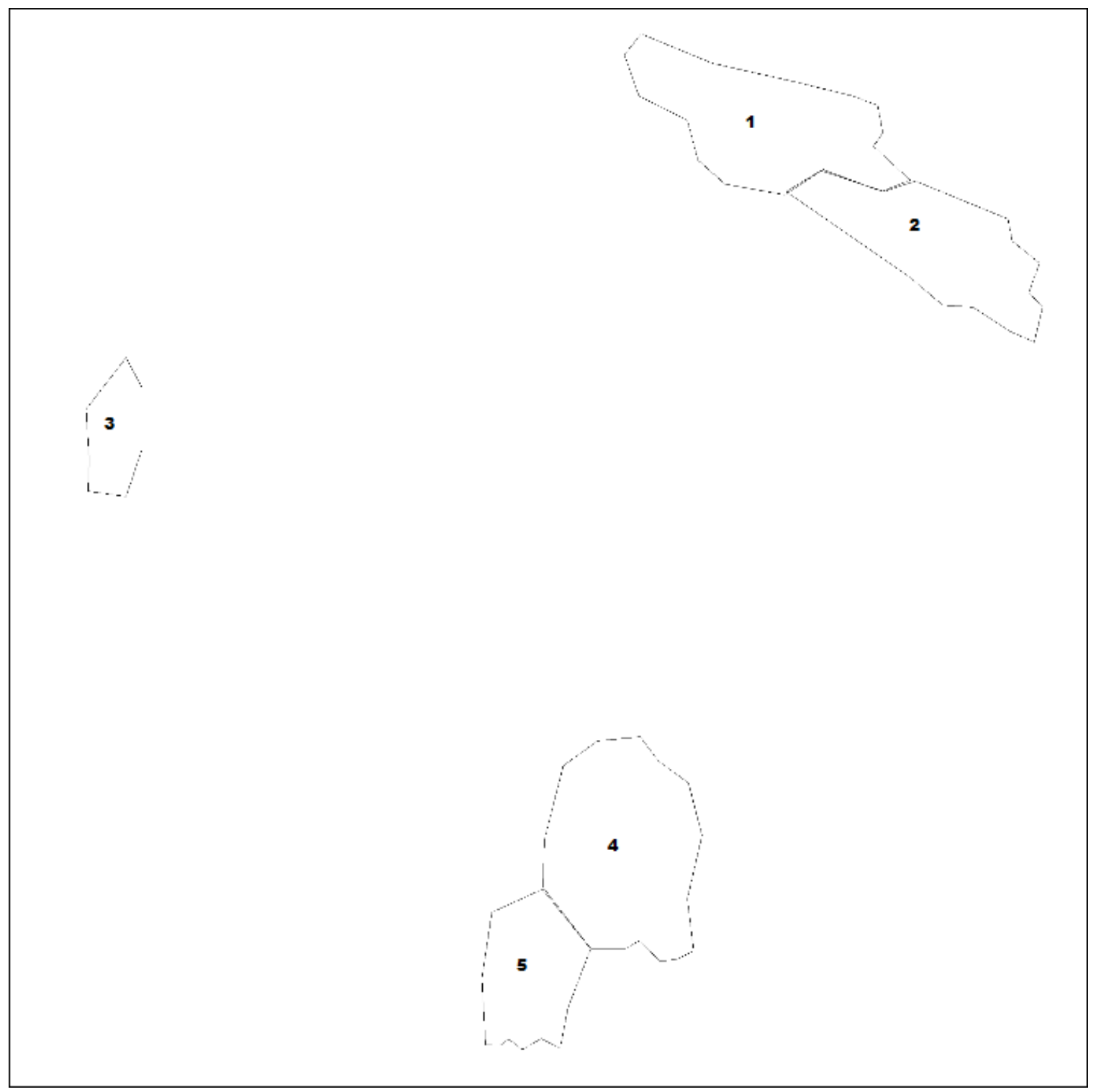

FIGURE A3.42- PRPE 20-1-3 ZO-1 Analysis 


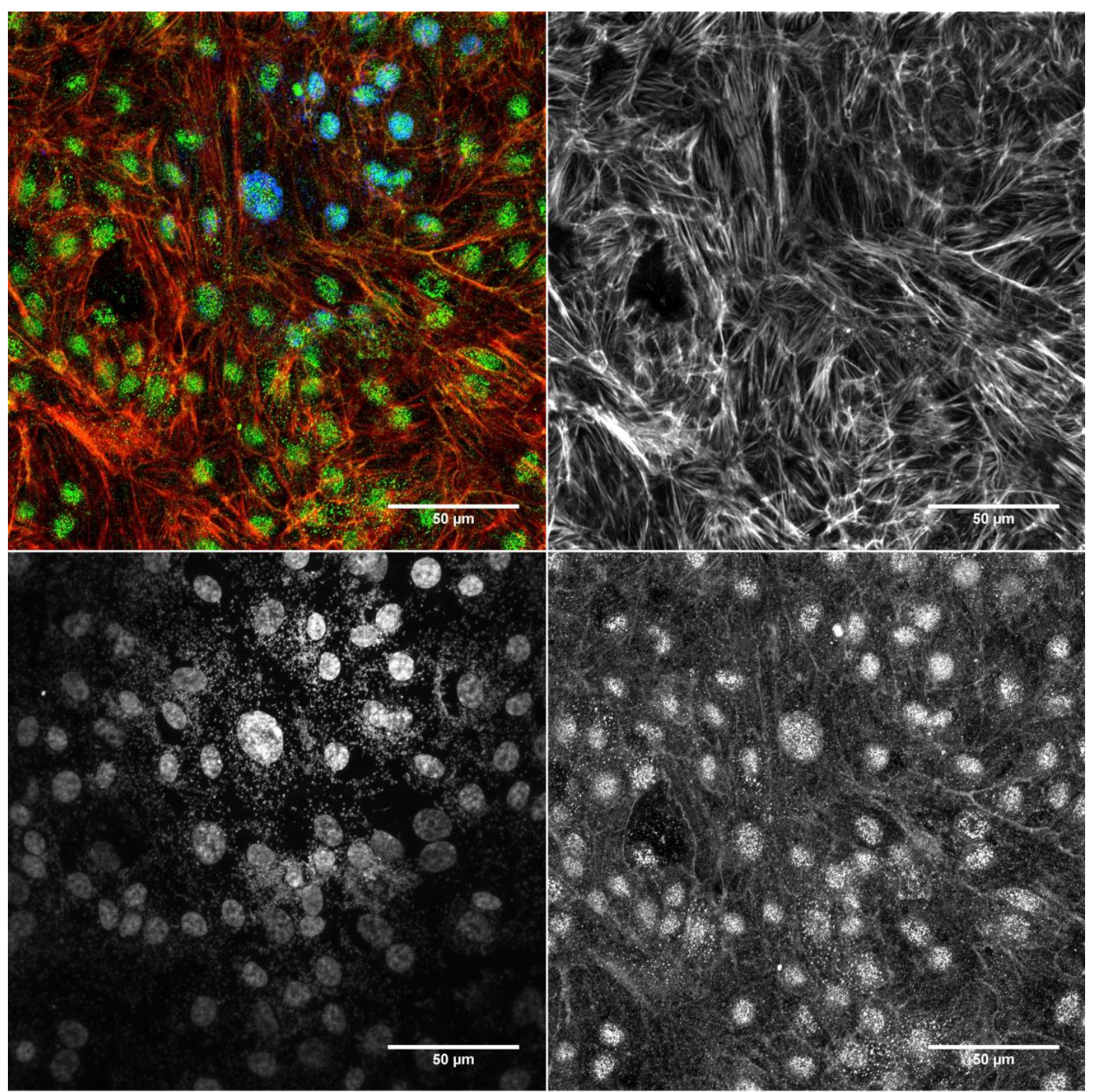

FIGURE A3.43- PRPE 20-3-1 (6/1/13) 


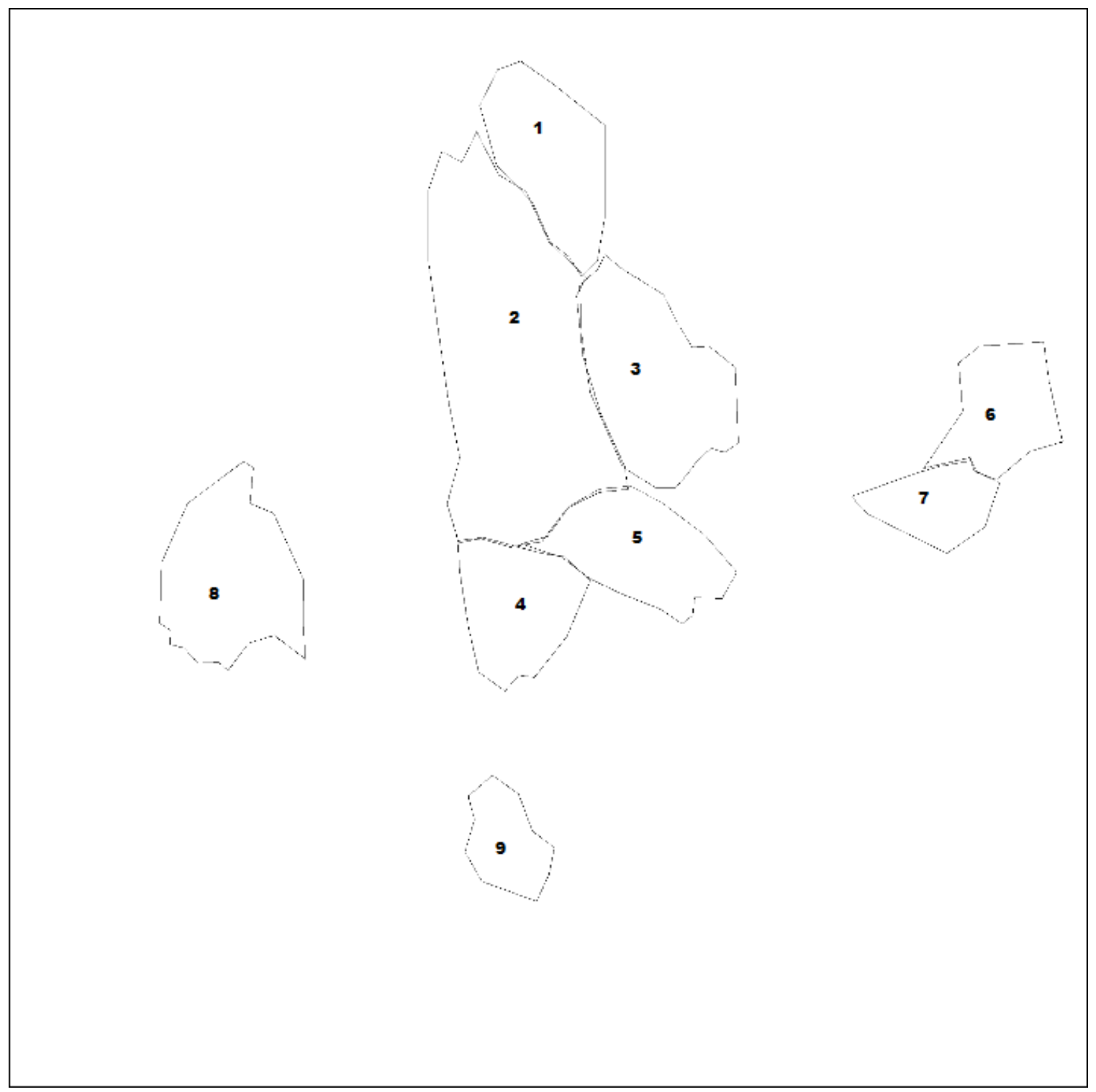

FIGURE A3.44- PRPE 20-3-1 ZO-1 Analysis 


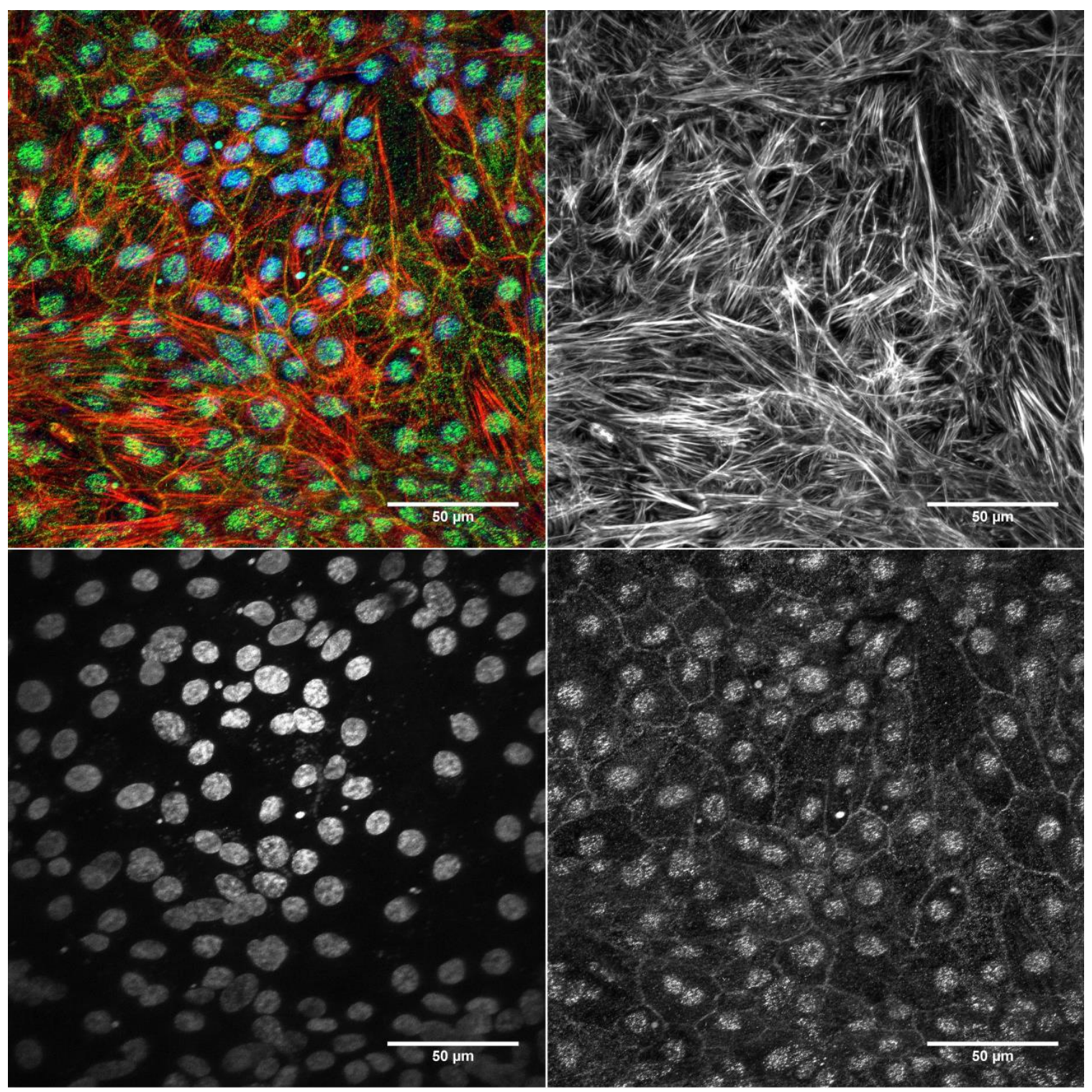

FIGURE A3.45- PRPE FN-1-1 (5/17/13) 


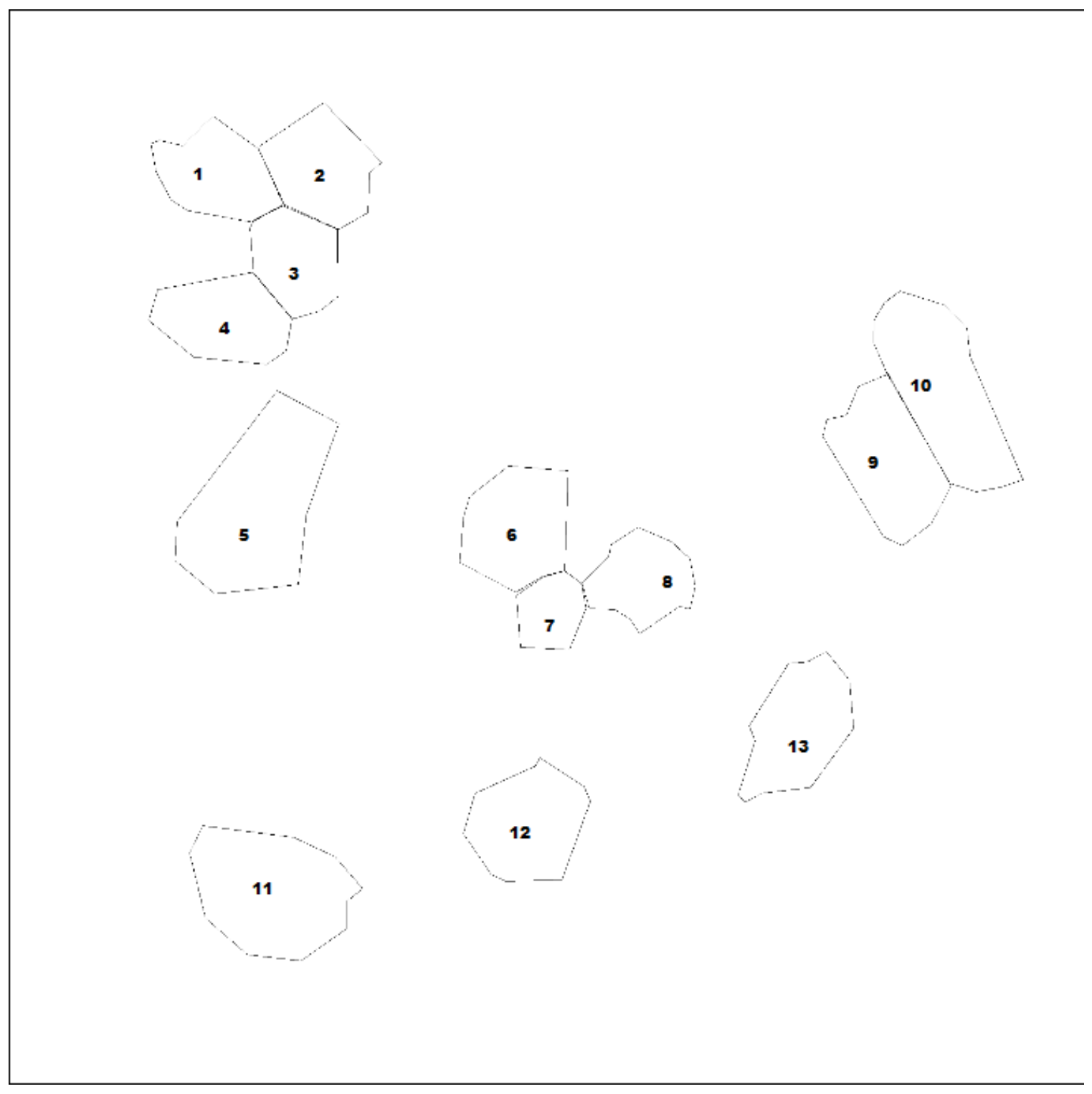

FIGURE A3.46- PRPE FN-1-1 ZO-1 Analysis 


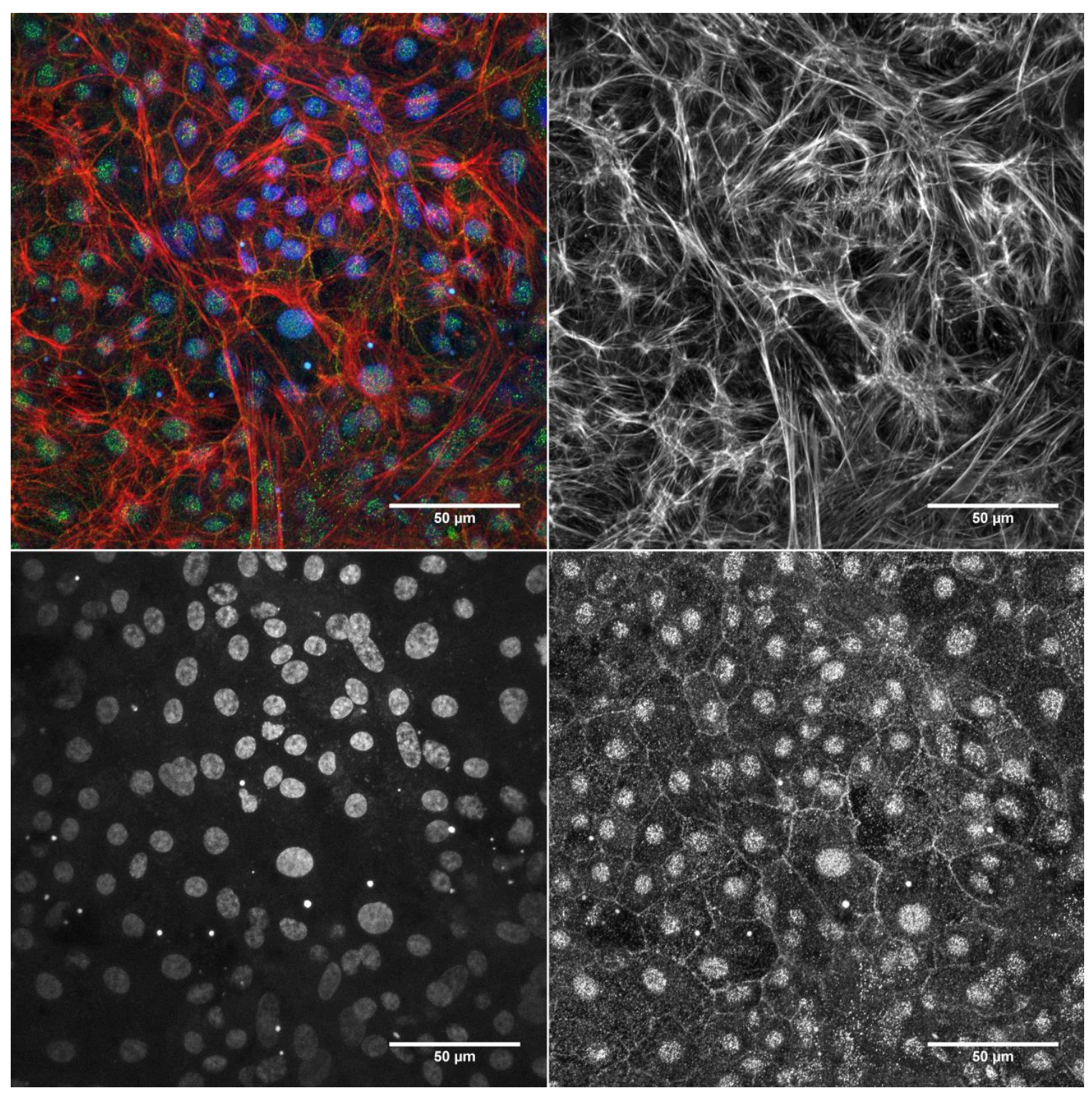

FIGURE A3.47- PRPE FN-2-1 (5/17/13) 


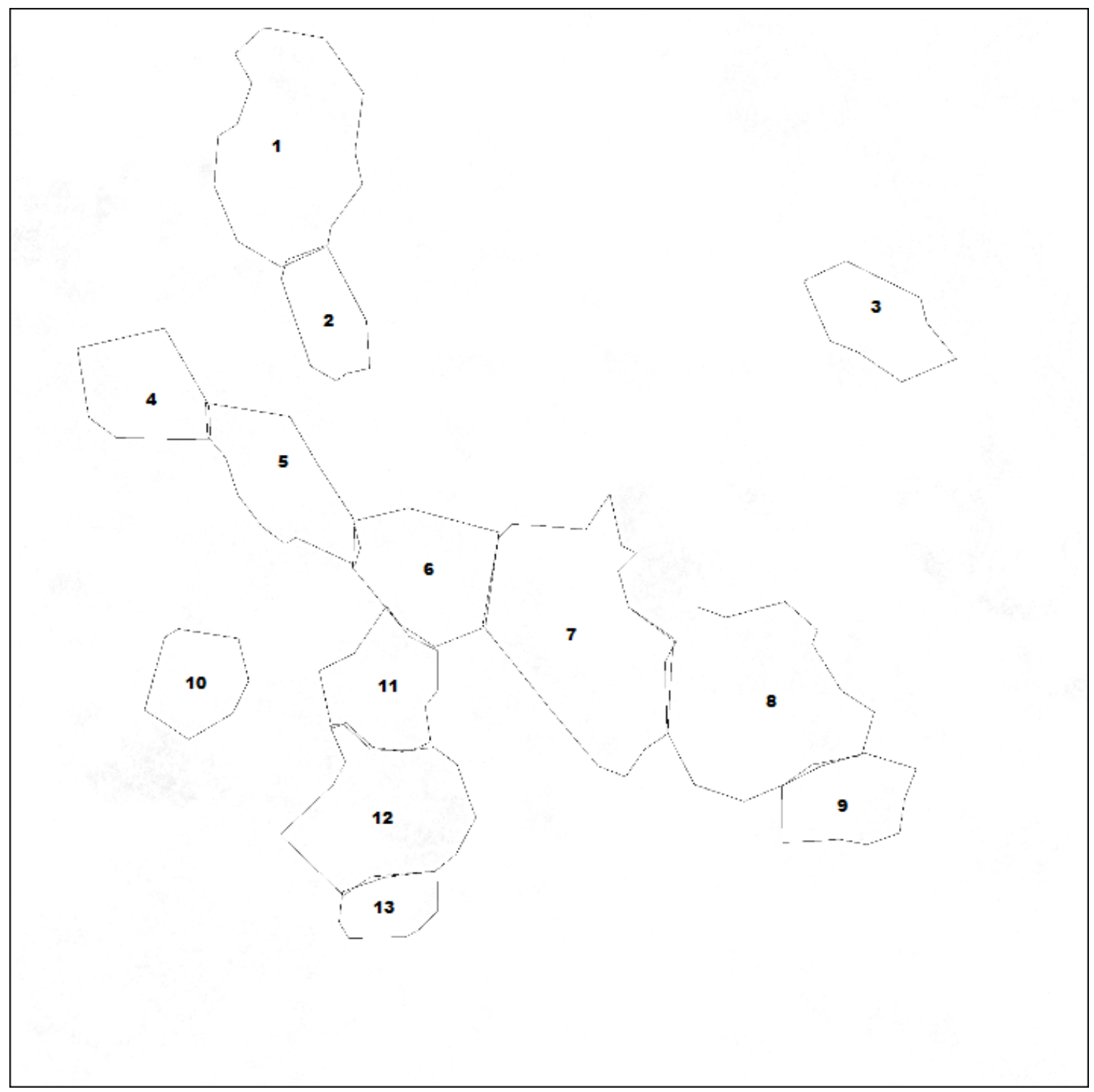

FIGURE A3.48- PRPE FN-2-1 ZO-1 Analysis 


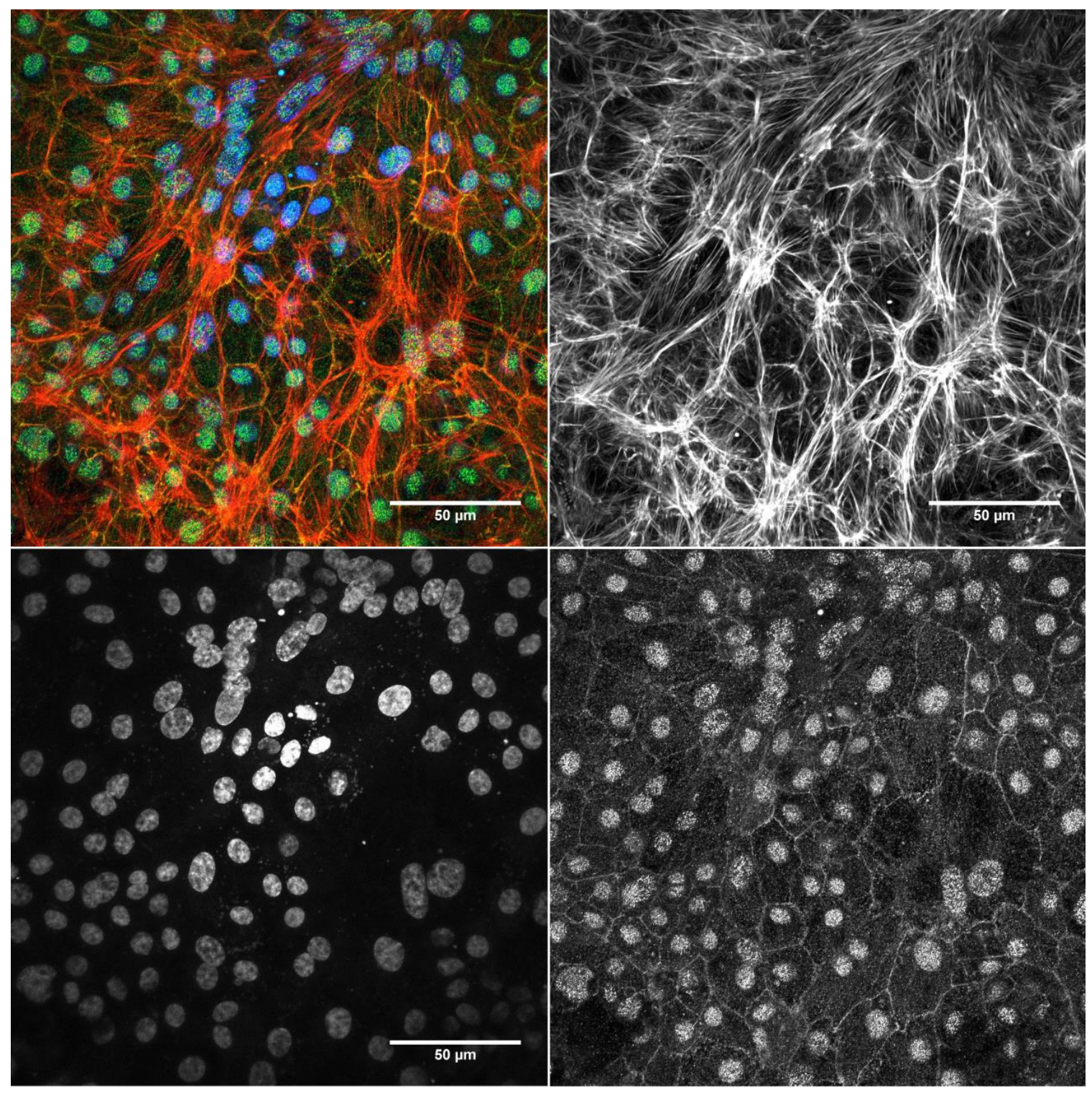

FIGURE A3.49- PRPE FN-1-2 (6/1/13) 


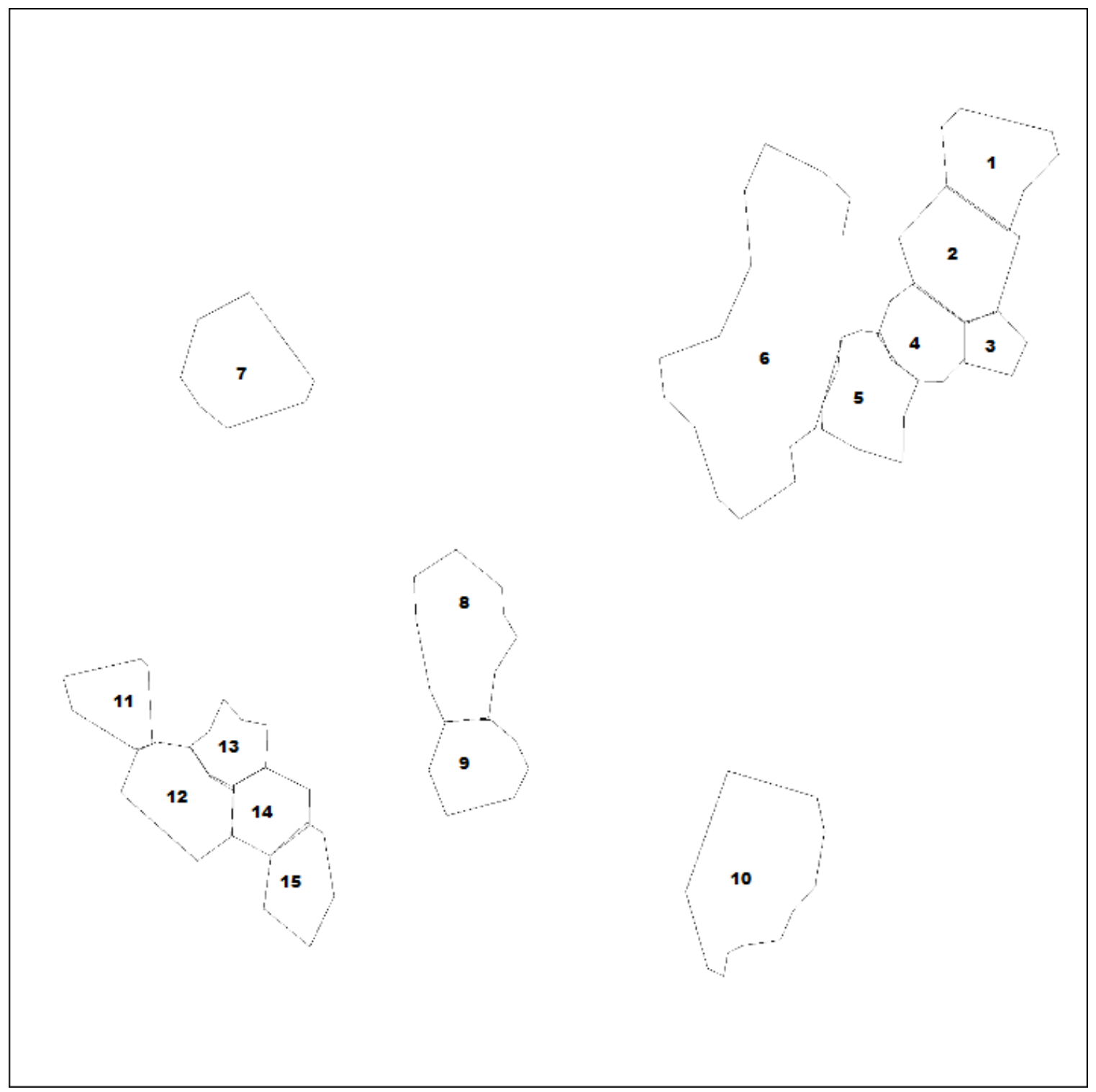

FIGURE A3.50- PRPE FN-1-2 ZO-1 Analysis 


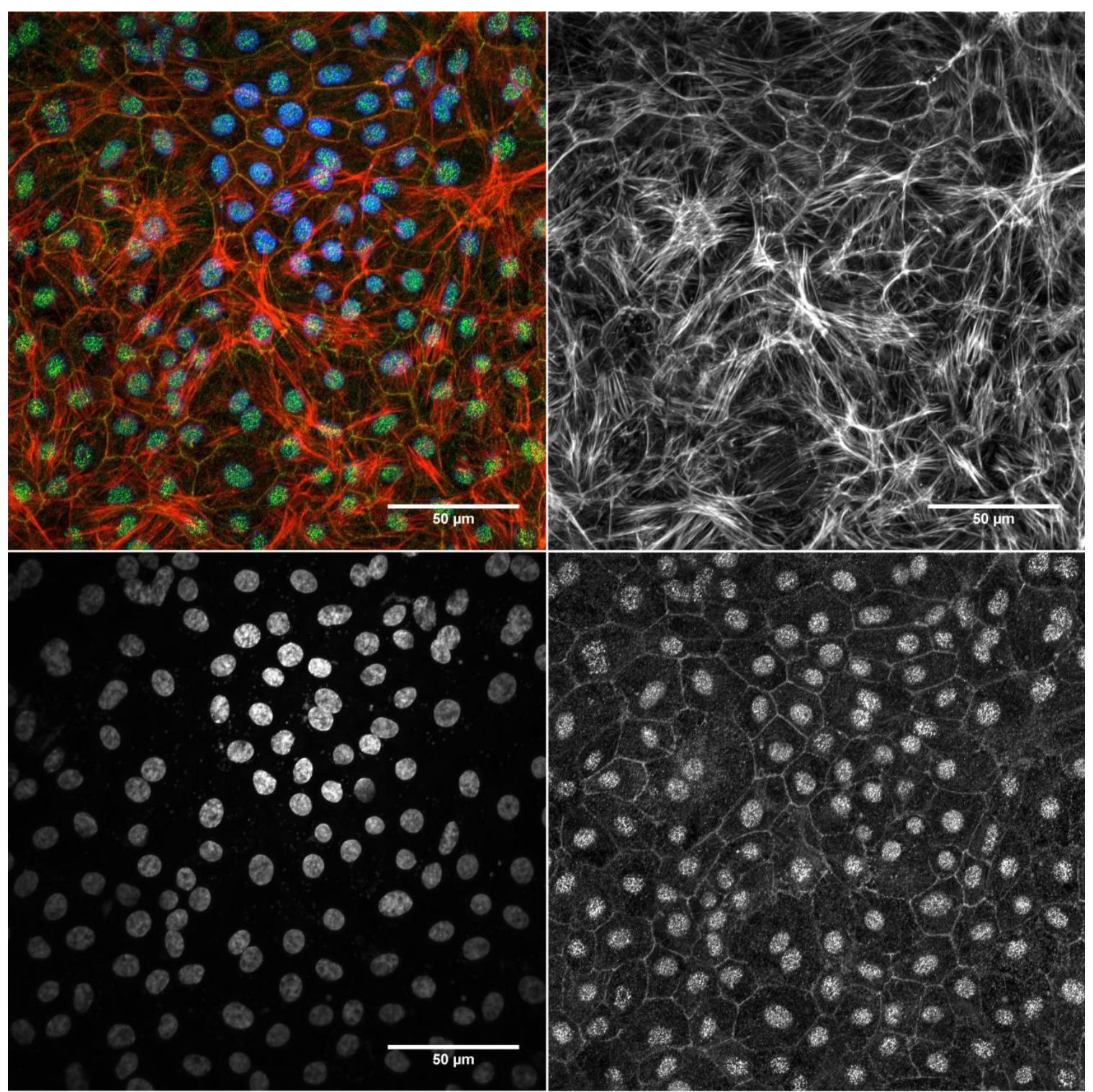

FIGURE A3.51- PRPE FN-2-2 (6/1/13) 


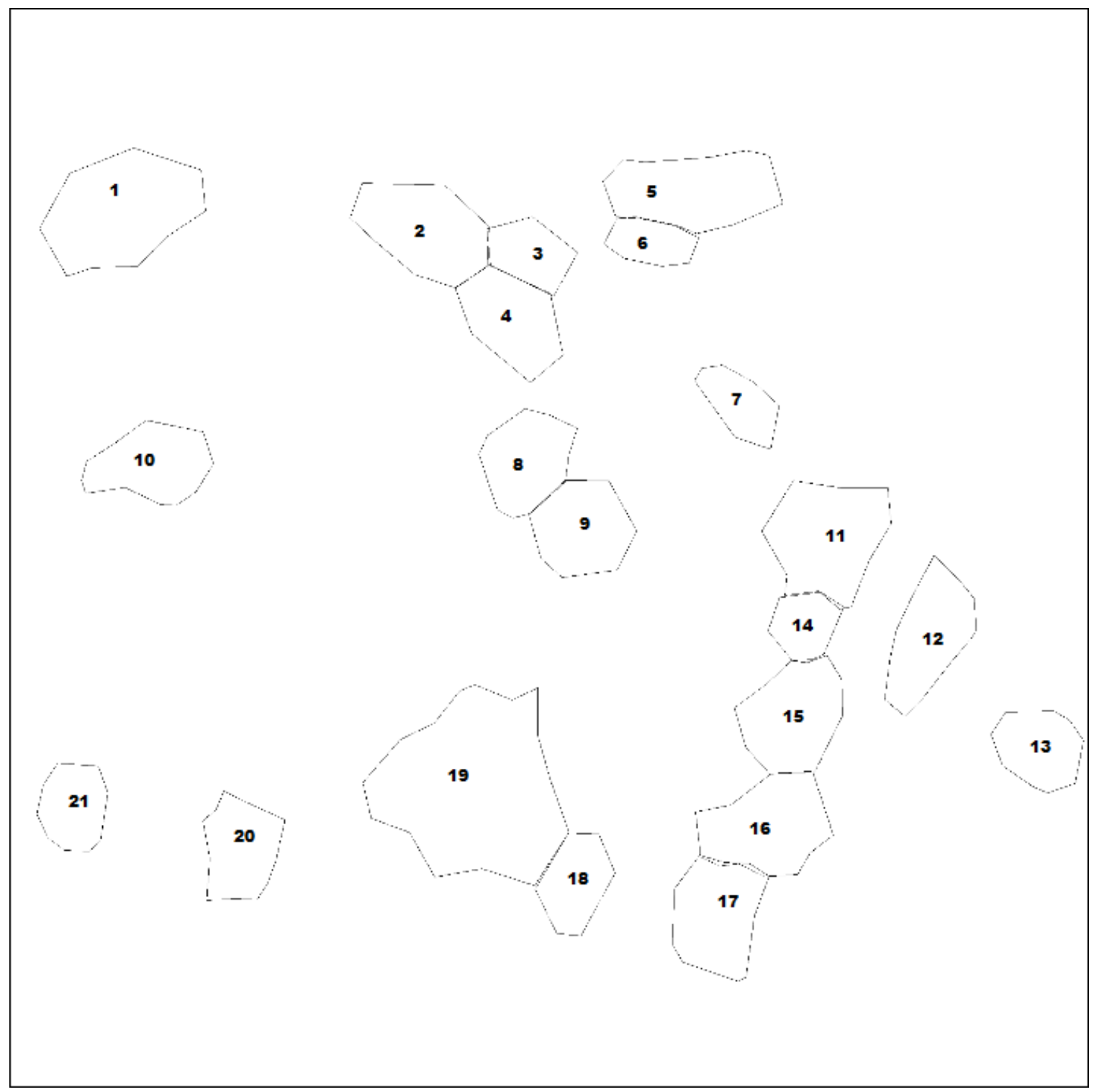

FIGURE A2.52- PRPE FN-2-2 ZO-1 Analysis 
VITA

Jeremy Phillips received his Bachelor's Degree of Science in Bioengineering with honors from the University of Louisville in 2012. Within that time he completed three coop rotations at Cook Inc. in Bloomington, IN. His first co-op consisted as an engineering testing lab co-op developing catheter testing equipment during the fall of 2010. Second, he served in production engineering focusing on the modification and improvement of bonding and catheter manufacturing during the summer of 2011. For his last co-op, he served as a production engineer working with the abdominal aortic aneurysms division to improve stent graft manufacturing during the spring of 2012. Jeremy also has two patent applications involving open surgical prosthesis deployment systems through a collaboration at Cook Inc. In between co-ops he also worked in the lab of biomimetic materials with Andrea Gobin, PhD. researching PEG modified proteins and peptides such as VEGF and RGDS. His other research also involved the development of macrogradient hydrogels, which led to writing and receiving funding for an undergraduate research grant. 